\title{
Iridium-Catalyzed Enantioselective Synthesis of $\alpha$-Chiral Bicyclo[1.1.1]pentanes by 1,3-Difunctionalization of [1.1.1]Propellane
}

Songjie Yu, ${ }^{\dagger}$ Changcheng Jing, ${ }^{\dagger}$ Adam Noble, and Varinder K. Aggarwal*

School of Chemistry, University of Bristol, Cantock's Close, Bristol BS8 1TS, U.K.

\section{Table of Contents}

I. General Information ...................................................................................................2

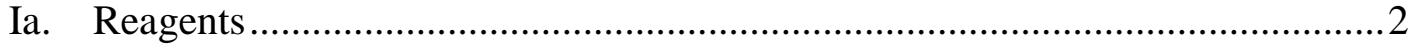

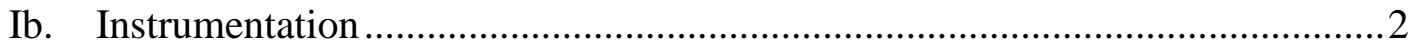

Ic. Safety Considerations ……………………………………………………...

II. General Procedures ...................................................................................................4

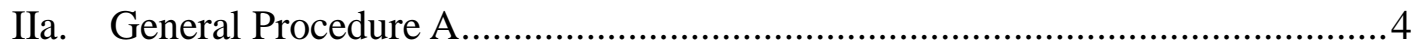

IIb. General Procedure B ……………………………………………….

III. Product Characterization ...................................................................................6

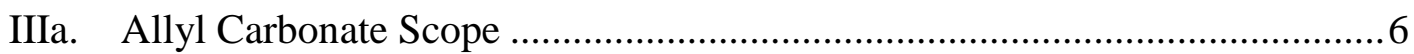

IIIb. Grignard Reagent Scope ...................................................................19

IIIc. Product Derivatizations ............................................................................29

IV. References ...............................................................................................................37

V. HPLC Spectra .........................................................................................................38

VI. NMR Spectra ..............................................................................................................68 


\section{General Information}

\section{Ia. Reagents}

All Grignard reagents and the $(S)$-phosphoramidite ligand $(\mathbf{L})$ were supplied by Sigma-Aldrich and were used as received. $\left[\operatorname{Ir}(\operatorname{cod}) \mathrm{Cl}_{2}\right.$ was purchased from Fisher Scientific. Dry solvents were purified by passing the degassed solvents $\left(\mathrm{N}_{2}\right)$ through a column of activated alumina. All reactions were performed in a nitrogen-filled glovebox or using Schlenk techniques. The allyl carbonate substrates were prepared according to the previous reports. ${ }^{1}$

\section{Ib. Instrumentation}

NMR $\left({ }^{1} \mathrm{H},{ }^{13} \mathrm{C}\right.$, and $\left.{ }^{19} \mathrm{~F}\right)$ spectra were recorded on Bruker nuclear resonance $400 \mathrm{MHz}$ spectrometer or Jeol ECS $400 \mathrm{MHz}$ spectrometer in the solvents indicated. Chemical shifts $(\delta)$ are given in parts per million (ppm).

HRMS analyses were performed a Bruker Daltonics Apex IV or micrOTOF instruments using electrospray ionization (ESI), electron impact ionization (EI) or atmospheric pressure chemical ionization (APCI).

IR spectra were recorded on a Perkin Elmer Spectrum One FT-IR as a thin film.

Optical rotations were measured in the indicated solvents using a Bellingham \& Stanley ADP 220 Polarimeter.

HPLC analyses were performed on Agilent 1100 system with Daicel Chiralpak IA and IB columns.

Gas chromatography-mass spectrometry (GC-MS) was performed on an Agilent 6890 Series GC and 5973 detector using a HP-5MS UI column.

Flash column chromatography was carried out using silica gel (Aldrich, silica gel 60, 40-63 $\mu \mathrm{m})$. 


\section{Ic. Safety Considerations}

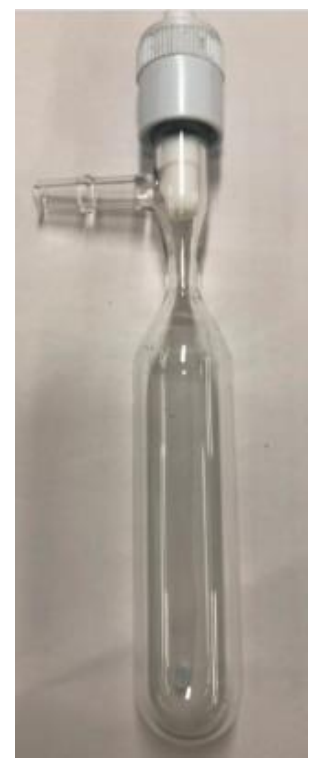

Unless otherwise stated, all of the Grignard reagent additions to [1.1.1]propellane were performed with no more than $2.5 \mathrm{~mL}$ of a diethyl ether solution in a $25 \mathrm{~mL}$ pressure tube fitted with a $\mathrm{J}$ Young stopcock [tube height $=10 \mathrm{~cm}(16 \mathrm{~cm}$ including stopcock); wall thickness $=2.1 \mathrm{~mm}$; outer diameter $=2.2 \mathrm{~cm}$; see picture on on left]. The glassware used was prepared by the glass workshop in the School of Chemistry at the University of Bristol, therefore it was not pressure rated. During our studies, no incidents occurred as a result of build-up of pressure in these reactions. Nonetheless, due to the low boiling points of diethyl ether and [1.1.1]propellane and risks associated with heating this mixture to $100{ }^{\circ} \mathrm{C}$ in a sealed vessel, all reactions were performed behind a blast shield. ${ }^{2}$

Under the reaction conditions, the maximum possible pressure within the system can be determined from the saturated vapor pressure of diethyl ether, which is 6.5 atm at $100{ }^{\circ} \mathrm{C}$. Therefore, pressure tubes that have a pressure rating of $10 \mathrm{~atm}$ (150 psi) should be used. To minimize the build-up of pressure during heating of the reactions, care was taken when submerging the reaction vessel in the oil bath to ensure that the oil level was only slightly higher than that of the internal solvent level (see picture on right). This maximizes air cooling of the head-space within the reaction vessel, therefore reducing the internal pressure. ${ }^{2}$

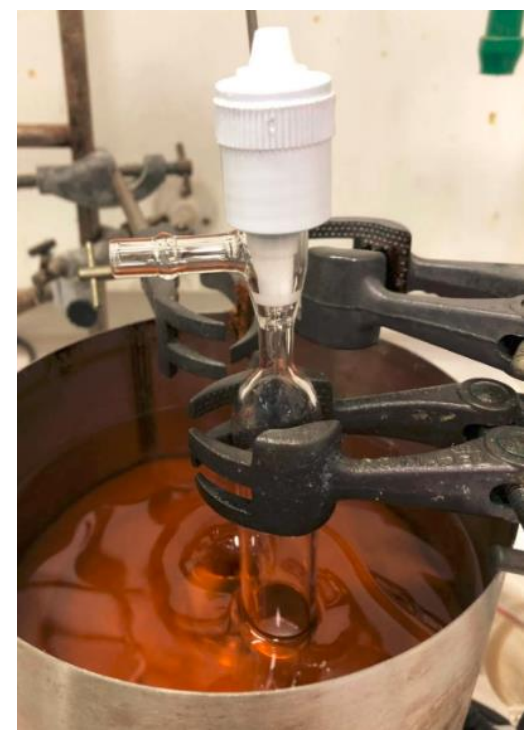




\section{General Procedures}

\section{IIa. General Procedure A}

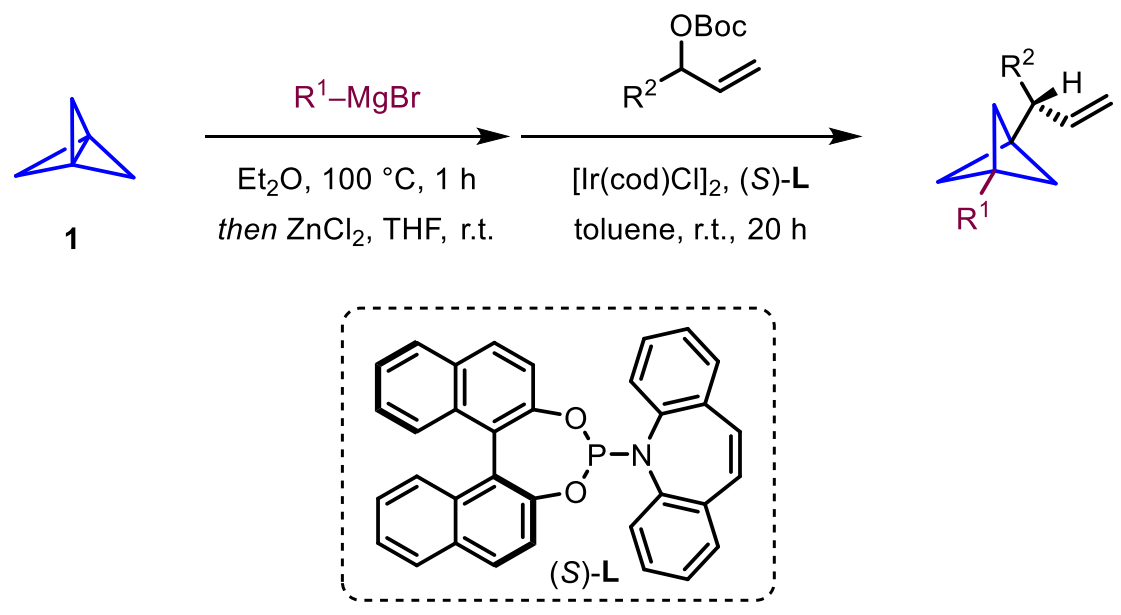

To a pressure tube was added aryl magnesium bromide (0.50-3.0 $\mathrm{M}$ in $\mathrm{Et}_{2} \mathrm{O}, 0.64$ mmol, 1.6 equiv) and [1.1.1]propellane (0.6-0.8 $\mathrm{M}_{\text {in }} \mathrm{Et}_{2} \mathrm{O}, 1.28 \mathrm{mmol}, 3.2$ equiv) at r.t. under an inert atmosphere. The pressure tube was sealed and the solution was heated to $100{ }^{\circ} \mathrm{C}$ and stirred for $1 \mathrm{~h}$. The solvent was removed under high vacuum before $\mathrm{ZnCl}_{2}$ ( $0.5 \mathrm{M}$ in THF, $0.80 \mathrm{mmol}, 2.0$ equiv) was added and the mixture stirred for $20 \mathrm{~min}$ at r.t. The solvent was removed under high vacuum and toluene $(1.0 \mathrm{~mL})$ was added. To a separate $5 \mathrm{~mL}$ vial was added $[\operatorname{Ir}(\operatorname{cod}) \mathrm{Cl}]_{2}(5.4 \mathrm{mg}, 0.0080 \mathrm{mmol}, 2.0$ mol\%), phosphoramidite ligand $(S)-\mathbf{L}(16.2 \mathrm{mg}, 0.032 \mathrm{mmol}, 8.0 \mathrm{~mol} \%)$ and toluene $(1.0 \mathrm{~mL})$ under an inert atmosphere, and the mixture was vigorously stirred for $20 \mathrm{~min}$ before adding the allyl carbonates $(0.40 \mathrm{mmol}, 1.0$ equiv). The resulting mixture of the catalyst and substrate was then added to the pressure tube and the resulting mixture was stirred at r.t. for $20 \mathrm{~h}$. Saturated aqueous $\mathrm{NH}_{4} \mathrm{Cl}(15 \mathrm{~mL})$ was added, the mixture was extracted with $\mathrm{Et}_{2} \mathrm{O}(3 \times 20 \mathrm{~mL})$, and the combined organic phases were dried over $\mathrm{Na}_{2} \mathrm{SO}_{4}$, filtered, and concentrated under reduced pressure. Purification by flash column chromatography using pentane as the eluent gave the corresponding product. The enantioselectivity was determined by chiral HPLC. 


\section{IIb. General Procedure B}
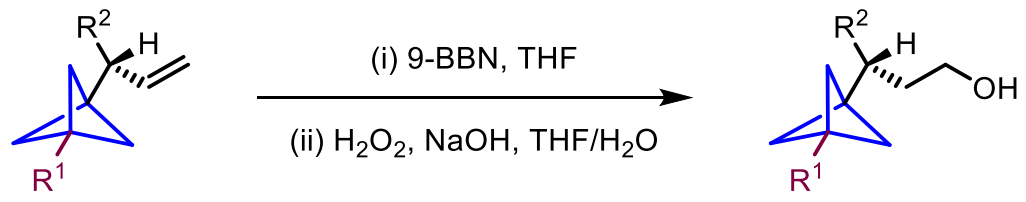

9-BBN (0.5 $\mathrm{M}$ in THF, 2 equiv.) was added a solution of the alkene (1.0 equiv.) in THF $(0.5 \mathrm{M})$ at $0{ }^{\circ} \mathrm{C}$. The mixture was stirred for $15 \mathrm{~min}$ at $0{ }^{\circ} \mathrm{C}$ and 6 hours at r.t. The reaction was cooled to $0{ }^{\circ} \mathrm{C}$, then $2 \mathrm{~N}$ aqueous $\mathrm{NaOH}(6.0$ equiv.) and $30 \%$ aqueous $\mathrm{H}_{2} \mathrm{O}_{2}$ (10 equiv.) were added. The reaction was allowed to warm to r.t. and stirring continued for $1 \mathrm{~h}$ before diluting with aqueous $\mathrm{NH}_{4} \mathrm{Cl}$ and extracting with $\mathrm{Et}_{2} \mathrm{O}$. The organic layer was dried over $\mathrm{MgSO}_{4}$, filtered, and concentrated under reduced pressure. The crude mixture was purified by flash column chromatography with hexanes/EtOAc as eluent to give the corresponding product. 


\section{Product Characterization}

IIIa. Allyl Carbonate Scope

(S)-1-(1-Phenylallyl)-3-(o-tolyl)bicyclo[1.1.1]pentane (4)

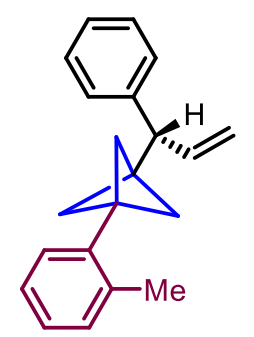

According to general procedure A: $o$-tolylmagnesium bromide $\left(2 \mathrm{M}\right.$ in $\mathrm{Et}_{2} \mathrm{O}, 0.64$ mmol), [1.1.1]propellane $(1.28 \mathrm{mmol})$ and tert-butyl (1-phenylallyl) carbonate (2a, $0.40 \mathrm{mmol})$ provided the product as a colorless oil $(85.5 \mathrm{mg}, 78 \%$ yield, $99 \%$ ee, pentane as an eluent).

${ }^{1} \mathrm{H}$ NMR (400 MHz, $\left.\mathrm{CDCl}_{3}\right) \delta 7.42-7.36(\mathrm{~m}, 2 \mathrm{H}), 7.32-7.24(\mathrm{~m}, 3 \mathrm{H}), 7.19-7.09$ (m, 4H), 6.21 (ddd, $J=17.0,10.3,8.4 \mathrm{~Hz}, 1 \mathrm{H}), 5.26-5.10(\mathrm{~m}, 2 \mathrm{H}), 3.56(\mathrm{~d}, J=8.3$ $\mathrm{Hz}, 1 \mathrm{H}), 2.41$ (s, 3H), 2.10-1.99 (m, 6H) (see spectrum) ${ }^{13} \mathrm{C} \mathrm{NMR}\left(100 \mathrm{MHz}, \mathrm{CDCl}_{3}\right)$ $\delta 142.0,138.5,138.4,136.9,130.4,128.3,128.0,127.7,126.8,126.2,125.7,115.9$, 51.9, 51.0, 43.4, 42.9, 20.8 (see spectrum) $.[\alpha]_{\mathrm{D}}^{25}=+6\left(c=1, \mathrm{CHCl}_{3}\right) . \mathrm{IR}$ (film) $v_{\max } / \mathrm{cm}^{-1}: 2970,1739,1365,1216,746$. HRMS (APCI): Calcd. for $\mathrm{C}_{21} \mathrm{H}_{23}\left([\mathrm{M}+\mathrm{H}]^{+}\right)$ $\mathrm{m} / \mathrm{z} 275.1794$, found $\mathrm{m} / \mathrm{z} 275.1790$.

HPLC conditions: Chiral column IB, $n$-hexane, flow rate $=0.7 \mathrm{~mL} / \mathrm{min}$, wavelength $=$ $210 \mathrm{~nm}, t_{\mathrm{R}}=9.8 \mathrm{~min}$ for major isomer, $t_{\mathrm{R}}=10.4 \mathrm{~min}$ for minor isomer (see spectrum) .

\section{(S)-1-(1-([1,1'-Biphenyl]-4-yl)allyl)-3-(o-tolyl)bicyclo[1.1.1]pentane (5)}

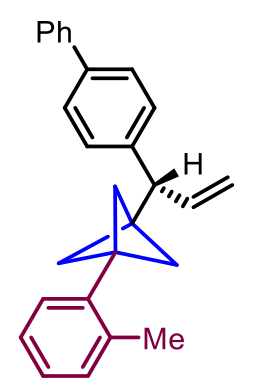

According to general procedure A: $o$-tolylmagnesium bromide ( $2 \mathrm{M}$ in $\mathrm{Et}_{2} \mathrm{O}, 0.64$ mmol), [1.1.1]propellane (1.28 mmol) and 1-([1,1'-biphenyl]-4-yl)allyl tert-butyl carbonate $(0.40 \mathrm{mmol})$ provided the product as a colorless oil $(113.4 \mathrm{mg}, 81 \%$ yield, pentane as an eluent).

${ }^{1} \mathrm{H}$ NMR (400 MHz, $\left.\mathrm{CDCl}_{3}\right) \delta 7.68-7.63(\mathrm{~m}, 2 \mathrm{H}), 7.63-7.58(\mathrm{~m}, 2 \mathrm{H}), 7.50-7.45$ 
(m, 2H), $7.40-7.34(\mathrm{~m}, 1 \mathrm{H}), 7.34-7.29(\mathrm{~m}, 2 \mathrm{H}), 7.18-7.08(\mathrm{~m}, 4 \mathrm{H}), 6.21$ (ddd, $J=$ $16.9,10.3,8.4 \mathrm{~Hz}, 1 \mathrm{H}), 5.21$ (ddd, $J=10.3,1.8,0.9 \mathrm{~Hz}, 1 \mathrm{H}), 5.19$ (ddd, $J=16.9,1.8$, $1.2 \mathrm{~Hz}, 1 \mathrm{H}), 3.59(\mathrm{~d}, J=8.4 \mathrm{~Hz}, 1 \mathrm{H}), 2.40(\mathrm{~s}, 3 \mathrm{H}), 2.10-2.01(\mathrm{~m}, 6 \mathrm{H})$ (see spectrum) ${ }^{13} \mathrm{C}$ NMR $\left(100 \mathrm{MHz}, \mathrm{CDCl}_{3}\right) \delta 141.13,141.05,139.1,138.5,138.3,136.9,130.5$, 128.8, 128.4, 127.8, 127.11, 127.08, 127.04, 126.8, 125.7, 116.0, 51.6, 51.1, 43.5, 42.8, 20.8 (see spectrum). HRMS (EI): Calcd. for $\mathrm{C}_{27} \mathrm{H}_{26}\left([\mathrm{M}]^{+}\right) \mathrm{m} / \mathrm{z} 350.2029$, found $\mathrm{m} / \mathrm{z} 350.2028$.

The ee of product 5 could not be determined by HPLC, therefore, the ee was determined after hydroboration/oxidation to alcohol $\mathbf{3 3}$.

\section{(S)-3-([1,1'-Biphenyl]-4-yl)-3-(3-(o-tolyl)bicyclo[1.1.1]pentan-1-yl)propan-1-ol}

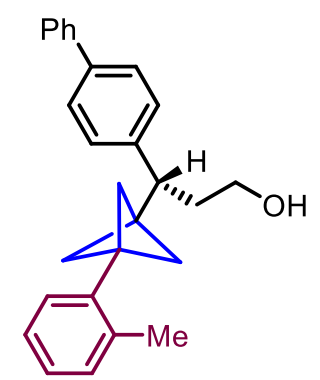

According to general procedure B, product 5 gave the corresponding alcohol $(54.4 \mathrm{mg}$, $93 \%$ yield, $99 \%$ ee, pentane/ $\mathrm{Et}_{2} \mathrm{O}(2 / 1)$ as an eluent).

${ }^{1} \mathrm{H}$ NMR (400 MHz, $\left.\mathrm{CDCl}_{3}\right) \delta 7.55-7.50(\mathrm{~m}, 2 \mathrm{H}), 7.49-7.45(\mathrm{~m}, 2 \mathrm{H}), 7.39-7.31$ $(\mathrm{m}, 2 \mathrm{H}), 7.28-7.20(\mathrm{~m}, 1 \mathrm{H}), 7.17-7.11(\mathrm{~m}, 2 \mathrm{H}), 7.05-6.93(\mathrm{~m}, 4 \mathrm{H}), 3.54$ (ddd, $J=$ $10.5,7.3,5.0 \mathrm{~Hz}, 1 \mathrm{H}), 3.45$ (ddd, $J=10.5,7.8,6.6 \mathrm{~Hz}, 1 \mathrm{H}), 2.89$ (dd, $J=11.0,4.4 \mathrm{~Hz}$, $1 \mathrm{H}), 2.26(\mathrm{~s}, 3 \mathrm{H}), 2.05-1.95(\mathrm{~m}, 1 \mathrm{H}), 1.93-1.84(\mathrm{~m}, 7 \mathrm{H}), 1.22$ (br. s, 1H) (see spectrum $).{ }^{13} \mathrm{C}$ NMR $\left(100 \mathrm{MHz}, \mathrm{CDCl}_{3}\right) \delta 141.0,140.9,139.1,138.5,136.9,130.4$, 128.8, 128.4, 127.7, 127.12, 127.05, 126.98, 126.8, 125.7, 61.4, 50.8, 43.6, 43.2, 42.7, $34.5,20.8$ (see spectrum) $.[\alpha]_{\mathrm{D}}^{25}=+7\left(c=1, \mathrm{CHCl}_{3}\right)$. IR (film) $v_{\max } / \mathrm{cm}^{-1}: 2970,2869$, 1738, 1366, 1217, 749. HRMS (ESI): Calcd. for $\mathrm{C}_{27} \mathrm{H}_{28} \mathrm{ONa}\left([\mathrm{M}+\mathrm{Na}]^{+}\right) \mathrm{m} / \mathrm{z} 391.2032$, found $\mathrm{m} / \mathrm{z} 391.2052$.

HPLC conditions: Chiral column IA, $n$-hexane $/ i-\mathrm{PrOH}=95: 5$, flow rate $=1.0$ $\mathrm{mL} / \mathrm{min}$, wavelength $=210 \mathrm{~nm}, t_{\mathrm{R}}=13.3 \mathrm{~min}$ for minor isomer, $t_{\mathrm{R}}=15.3 \mathrm{~min}$ for major isomer (see spectrum). 


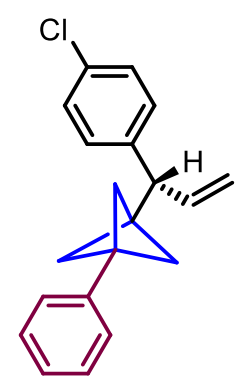

According to general procedure A: phenylmagnesium bromide ( $3 \mathrm{M}$ in $\mathrm{Et}_{2} \mathrm{O}, 0.64$ mmol), [1.1.1]propellane (1.28 mmol) and tert-butyl (1-(4-chlorophenyl)allyl) carbonate $(0.40 \mathrm{mmol})$ provided the product as a colorless oil $(87.0 \mathrm{mg}, 74 \%$ yield, $98 \%$ ee, pentane as an eluent).

${ }^{1} \mathrm{H}$ NMR (400 MHz, $\left.\mathrm{CDCl}_{3}\right) \delta 7.38-7.30(\mathrm{~m}, 4 \mathrm{H}), 7.27-7.20(\mathrm{~m}, 3 \mathrm{H}), 7.20-7.15$ $(\mathrm{m}, 2 \mathrm{H}), 6.13$ (ddd, $J=17.0,10.3,8.2 \mathrm{~Hz}, 1 \mathrm{H}), 5.20$ (ddd, $J=10.3,1.7,0.9 \mathrm{~Hz}, 1 \mathrm{H}$ ), 5.15 (ddd, $J=17.0,1.7,1.2 \mathrm{~Hz}, 1 \mathrm{H}), 3.53(\mathrm{~d}, J=8.1 \mathrm{~Hz}, 1 \mathrm{H}), 2.01-1.84(\mathrm{~m}, 6 \mathrm{H})$ (see spectrum) ${ }^{13} \mathrm{C}$ NMR $\left(100 \mathrm{MHz}, \mathrm{CDCl}_{3}\right) \delta 141.0,140.5,137.8,132.0,129.4$, $128.5,128.1,126.4,126.0,116.3,51.2,50.9,42.5,41.5$ (see spectrum $) .[\alpha]_{\mathrm{D}}^{25}=+7(c$ $=1, \mathrm{CHCl}_{3}$ ). IR (film) $v_{\max } / \mathrm{cm}^{-1}: 2970,1738,1217,697$. HRMS (APCI): Calcd. for $\mathrm{C}_{20} \mathrm{H}_{19} \mathrm{Cl}\left([\mathrm{M}+\mathrm{H}]^{+}\right) \mathrm{m} / \mathrm{z} 295.1248$, found $\mathrm{m} / \mathrm{z} 295.1249$.

HPLC conditions: Chiral column IA, $n$-hexane, flow rate $=1.0 \mathrm{~mL} / \mathrm{min}$, wavelength $=$ $210 \mathrm{~nm}, t_{\mathrm{R}}=9.5 \mathrm{~min}$ for minor isomer, $t_{\mathrm{R}}=14.6 \mathrm{~min}$ for major isomer (see spectrum) .

(S)-1-(o-Tolyl)-3-(1-(4-(trifluoromethyl)phenyl)allyl)bicyclo[1.1.1]pentane (7)

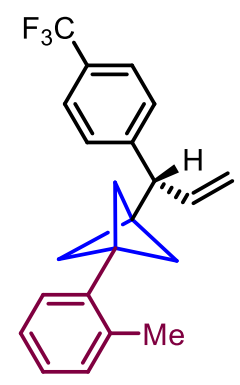

According to general procedure A: $o$-tolylmagnesium bromide ( $2 \mathrm{M}$ in $\mathrm{Et}_{2} \mathrm{O}, 0.64$ mmol), [1.1.1]propellane $(1.28 \quad \mathrm{mmol})$ and tert-butyl (1-(4-(trifluoromethyl)phenyl)allyl) carbonate $(0.40 \mathrm{mmol})$ provided the product as a colorless oil ( $87.4 \mathrm{mg}, 67 \%$ yield, $99 \%$ ee, pentane as an eluent).

${ }^{1} \mathrm{H}$ NMR (400 MHz, $\left.\mathrm{CDCl}_{3}\right) \delta 7.73-7.52(\mathrm{~m}, 2 \mathrm{H}), 7.34(\mathrm{~d}, J=8.2 \mathrm{~Hz}, 2 \mathrm{H}), 7.18-$ $7.13(\mathrm{~m}, 2 \mathrm{H}), 7.13-7.07(\mathrm{~m}, 2 \mathrm{H}), 6.16(\mathrm{ddd}, J=17.0,10.3,8.3 \mathrm{~Hz}, 1 \mathrm{H}), 5.22$ (ddd, $J$ $=10.3,1.6,0.9 \mathrm{~Hz}, 1 \mathrm{H}), 5.16(\mathrm{ddd}, J=17.1,1.6,1.2 \mathrm{~Hz}, 1 \mathrm{H}), 3.61(\mathrm{~d}, J=8.3 \mathrm{~Hz}$, $1 \mathrm{H}), 2.38(\mathrm{~s}, 3 \mathrm{H}), 2.11-1.88(\mathrm{~m}, 6 \mathrm{H})$ (see spectrum $).{ }^{19} \mathrm{~F}$ NMR $\left(377 \mathrm{MHz}, \mathrm{CDCl}_{3}\right) \delta$ 
-62.2 (s) (see spectrum). ${ }^{13} \mathrm{C}$ NMR (100 MHz, $\left.\mathrm{CDCl}_{3}\right) \delta 146.2,138.3,137.5,136.9$, 130.6, 128.7 (q, $J=32.3 \mathrm{~Hz}), 128.4,127.8,127.0,125.9,125.4(\mathrm{q}, J=3.8 \mathrm{~Hz}), 123.1$ $(\mathrm{q}, J=271.8 \mathrm{~Hz}), 116.8,51.9,51.1,43.7,42.7,20.8$ (see spectrum $) .[\alpha]_{\mathrm{D}}^{25}=+7(c=1$, $\mathrm{CHCl}_{3}$ ). IR (film) $v_{\max } / \mathrm{cm}^{-1}: 2966,2928,2908,2870,1619,1325,1256,1165,1124$, 1068, 1018, 920, 853, 745. HRMS (EI): Calcd. for $\mathrm{C}_{22} \mathrm{H}_{21} \mathrm{~F}_{3}\left(\left[\mathrm{M}-\mathrm{CH}_{3}\right]^{+}\right) \mathrm{m} / \mathrm{z}$ 327.1355 , found $\mathrm{m} / \mathrm{z} 327.1353$.

HPLC conditions: Chiral column IB (two IB columns), $n$-hexane, flow rate $=0.5$ $\mathrm{mL} / \mathrm{min}$, wavelength $=210 \mathrm{~nm}, t_{\mathrm{R}}=31.1 \mathrm{~min}$ for major isomer, $t_{\mathrm{R}}=30.5 \mathrm{~min}$ for minor isomer (see spectrum).

\section{(S)-1-(1-(4-(Phenylsulfonyl)phenyl)allyl)-3-(o-tolyl)bicyclo[1.1.1]pentane (8)}

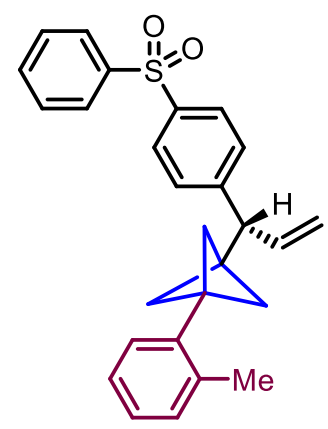

According to general procedure A: $o$-tolylmagnesium bromide (2 $\mathrm{M}$ in $\mathrm{Et}_{2} \mathrm{O}, 0.64$ mmol), [1.1.1]propellane $(1.28 \quad \mathrm{mmol})$ and tert-butyl (1-(4-(phenylsulfonyl)phenyl)allyl) carbonate $(0.40 \mathrm{mmol})$ provided the product as a colorless oil (125.9 mg, 76\% yield, pentane/ $\mathrm{Et}_{2} \mathrm{O}$ (4/1) as an eluent).

${ }^{1} \mathrm{H}$ NMR $\left(400 \mathrm{MHz}, \mathrm{CDCl}_{3}\right) \delta 8.02-7.96(\mathrm{~m}, 2 \mathrm{H}), 7.95-7.90(\mathrm{~m}, 2 \mathrm{H}), 7.63-7.57$ $(\mathrm{m}, 1 \mathrm{H}), 7.56-7.51(\mathrm{~m}, 2 \mathrm{H}), 7.38-7.32(\mathrm{~m}, 2 \mathrm{H}), 7.16-7.03(\mathrm{~m}, 4 \mathrm{H}), 6.10(\mathrm{ddd}, J=$ $17.0,10.3,8.4 \mathrm{~Hz}, 1 \mathrm{H}), 5.19(\mathrm{dt}, J=10.2,1.1 \mathrm{~Hz}, 1 \mathrm{H}), 5.12(\mathrm{dt}, J=17.0,1.3 \mathrm{~Hz}, 1 \mathrm{H})$, $3.58(\mathrm{~d}, J=8.4 \mathrm{~Hz}, 1 \mathrm{H}), 2.35(\mathrm{~s}, 3 \mathrm{H}), 2.04-1.90(\mathrm{~m}, 6 \mathrm{H})$ (see spectrum $).{ }^{13} \mathrm{C} \mathrm{NMR}$ $\left(100 \mathrm{MHz}, \mathrm{CDCl}_{3}\right) \delta 148.0,141.8,139.4,137.9,136.9,136.8,1331,130.5,129.3$, 128.8, 127.8, 127.7, 127.6, 126.9, 125.7, 117.0, 51.8, 51.0, 43.6, 42.4, 20.7 (see spectrum). HRMS (APCI): Calcd. for $\mathrm{C}_{27} \mathrm{H}_{27} \mathrm{O}_{2} \mathrm{~S}\left([\mathrm{M}+\mathrm{H}]^{+}\right) \mathrm{m} / \mathrm{z} 415.1732$, found $\mathrm{m} / \mathrm{z}$ 415.1737.

The ee of product $\mathbf{8}$ could not be determined by HPLC, therefore, the ee was determined after hydroboration/oxidation to alcohol $\mathbf{8 a}$. 


\section{1-yl)propan-1-ol (8a)}

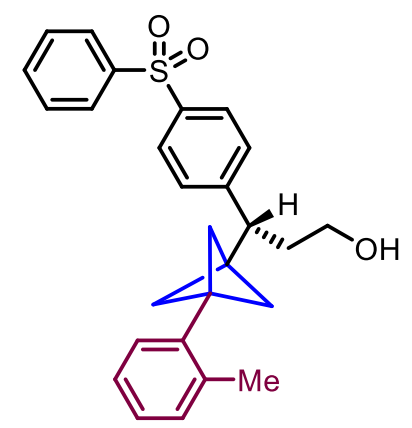

According to general procedure B, product 8 gave the corresponding alcohol $(57.7 \mathrm{mg}$, $89 \%$ yield, $>99 \%$ ee, pentane/ $\mathrm{Et}_{2} \mathrm{O}(1 / 1)$ as an eluent).

${ }^{1} \mathrm{H}$ NMR (400 MHz, $\left.\mathrm{CDCl}_{3}\right) \delta 8.00-7.94(\mathrm{~m}, 2 \mathrm{H}), 7.92-7.86(\mathrm{~m}, 2 \mathrm{H}), 7.61-7.48$ (m, 3H), $7.32-7.27(\mathrm{~m}, 2 \mathrm{H}), 7.13-7.00(\mathrm{~m}, 4 \mathrm{H}), 3.55$ (ddd, $J=11.5,7.2,4.8 \mathrm{~Hz}$, $1 \mathrm{H}), 3.41$ (ddd, $J=10.4,8.1,6.5 \mathrm{~Hz}, 1 \mathrm{H}), 3.04(\mathrm{dd}, J=10.9,4.4 \mathrm{~Hz}, 1 \mathrm{H}), 2.31$ (s, $3 \mathrm{H}), 2.13-2.01(\mathrm{~m}, 1 \mathrm{H}), 1.98-1.85(\mathrm{~m}, 7 \mathrm{H})$ (see spectrum $).{ }^{13} \mathrm{C}$ NMR $(100 \mathrm{MHz}$, $\left.\mathrm{CDCl}_{3}\right) \delta 148.3,141.8,139.6,138.0,136.9,133.2,130.5,129.4,129.0,127.9,127.8$, $127.7,127.0,125.8,60.8,50.8,43.4,43.1,42.9,34.2,20.8$ (see spectrum). $[\alpha]_{\mathrm{D}}{ }^{25}=+5$ $\left(c=1, \mathrm{CHCl}_{3}\right)$. IR (film) $v_{\max } / \mathrm{cm}^{-1}: 2970,1738,1366,753$. HRMS (ESI): Calcd. for $\mathrm{C}_{27} \mathrm{H}_{29} \mathrm{O}_{3} \mathrm{~S}\left([\mathrm{M}+\mathrm{H}]^{+}\right) \mathrm{m} / \mathrm{z} 433.1832$, found $\mathrm{m} / \mathrm{z} 433.1831$.

HPLC conditions: Chiral column IA, $n$-hexane $/ i$-PrOH $=85 / 15$, flow rate $=1 \mathrm{~mL} / \mathrm{min}$, wavelength $=210 \mathrm{~nm}, t_{\mathrm{R}}=19.9 \mathrm{~min}$ for major isomer, $t_{\mathrm{R}}=23.1 \mathrm{~min}$ for minor isomer (see spectrum).

\section{Methyl (S)-4-(1-(3-(o-tolyl)bicyclo[1.1.1]pentan-1-yl)allyl)benzoate (9)}

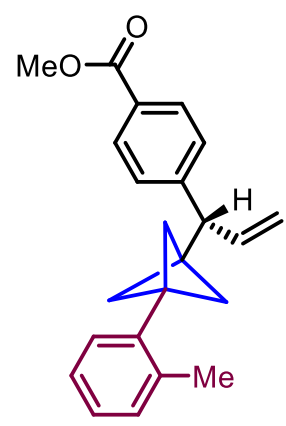

According to general procedure A: $o$-tolylmagnesium bromide $\left(2 \mathrm{M}\right.$ in $\mathrm{Et}_{2} \mathrm{O}, 0.64$ mmol), [1.1.1]propellane $(1.28 \quad \mathrm{mmol})$ and methyl 4-(1-((tert-butoxycarbonyl)oxy)allyl)-benzoate $(0.40 \mathrm{mmol})$ provided the product as a white solid (90.3 mg, 68\% yield, $89 \%$ ee, pentane/ $\mathrm{Et}_{2} \mathrm{O}$ (10/1) as an eluent).

${ }^{1} \mathrm{H}$ NMR (400 MHz, $\left.\mathrm{CDCl}_{3}\right) \delta 8.21-7.94(\mathrm{~m}, 2 \mathrm{H}), 7.35$ - 7.27 (m, 2H), $7.19-7.12$ 
(m, 2H), $7.19-7.13(\mathrm{~m}, 2 \mathrm{H}), 6.18(\mathrm{ddd}, J=17.0,10.3,8.3 \mathrm{~Hz}, 1 \mathrm{H}), 5.22(\mathrm{ddd}, J=$ 10.3, 1.7, $0.9 \mathrm{~Hz}, 1 \mathrm{H}), 5.17$ (ddd, $J=17.0,1.7,1.1 \mathrm{~Hz}, 1 \mathrm{H}), 3.95$ (s, 3H), 3.61 (d, $J=$ $8.3 \mathrm{~Hz}, 1 \mathrm{H}), 2.38(\mathrm{~s}, 3 \mathrm{H}), 2.14-1.89(\mathrm{~m}, 6 \mathrm{H})$ (see spectrum) ${ }^{13} \mathrm{C}$ NMR $(100 \mathrm{MHz}$, $\left.\mathrm{CDCl}_{3}\right) \delta 167.1,147.4,138.3,137.5,136.8,130.5,129.8,128.3,128.1,127.7,126.9$, $125.8,116.6,52.1,52.0,51.1,43.6,42.6,20.8$ (see spectrum $) .[\alpha]_{\mathrm{D}}^{25}=+11(c=1$, $\mathrm{CHCl}_{3}$ ). IR (film) $v_{\max } / \mathrm{cm}^{-1}: 2966,2908,2868,1723,1610,1435,1278,1256,1180$, 1111, 1019, 917, 749. HRMS (ESI): Calcd. for $\mathrm{C}_{23} \mathrm{H}_{25} \mathrm{O}_{2}\left([\mathrm{M}+\mathrm{H}]^{+}\right) \mathrm{m} / \mathrm{z} 333.1849$, found $\mathrm{m} / \mathrm{z} 333.1846$.

HPLC conditions: Chiral column IA, $n$-hexane $/ i-\mathrm{PrOH}=98: 2$, flow rate $=0.9$ $\mathrm{mL} / \mathrm{min}$, wavelength $=210 \mathrm{~nm}, t_{\mathrm{R}}=9.8 \mathrm{~min}$ for major isomer, $t_{\mathrm{R}}=8.3 \mathrm{~min}$ for minor isomer (see spectrum).

\section{(S)-1-(1-(3-Methoxyphenyl)allyl)-3-(o-tolyl)bicyclo[1.1.1]pentane (10)}

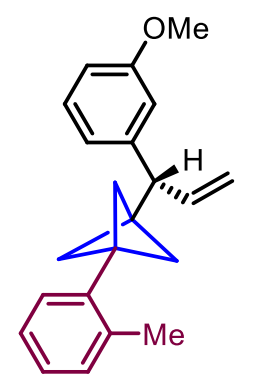

According to general procedure A: $o$-tolylmagnesium bromide ( $2 \mathrm{M}$ in $\mathrm{Et}_{2} \mathrm{O}, 0.64$ $\mathrm{mmol})$, [1.1.1]propellane (1.28 $\mathrm{mmol})$ and tert-butyl (1-(3-methoxyphenyl)allyl) carbonate $(0.40 \mathrm{mmol})$ provided the product as a colorless oil $(86.3 \mathrm{mg}, 71 \%$ yield, $>99 \%$ ee, pentane/ $\mathrm{Et}_{2} \mathrm{O}(20 / 1)$ as an eluent).

${ }^{1} \mathrm{H}$ NMR $\left(400 \mathrm{MHz}, \mathrm{CDCl}_{3}\right) \delta 7.29(\mathrm{td}, J=7.8,0.6 \mathrm{~Hz}, 1 \mathrm{H}), 7.19-7.07(\mathrm{~m}, 4 \mathrm{H})$, $6.90-6.75(\mathrm{~m}, 3 \mathrm{H}), 6.16(\mathrm{ddd}, J=16.9,10.3,8.4 \mathrm{~Hz}, 1 \mathrm{H}), 5.18(\mathrm{ddd}, J=10.3,1.8$, $0.8 \mathrm{~Hz}, 1 \mathrm{H}), 5.16$ (ddd, $J=16.9,1.8,1.1 \mathrm{~Hz}, 1 \mathrm{H}), 3.85$ (s, 3H), 3.51 (d, $J=8.4 \mathrm{~Hz}$, $1 \mathrm{H}), 2.39(\mathrm{~s}, 3 \mathrm{H}), 2.11-1.94(\mathrm{~m}, 6 \mathrm{H})$ (see spectrum) ${ }^{13} \mathrm{C} \mathrm{NMR}\left(100 \mathrm{MHz}, \mathrm{CDCl}_{3}\right) \delta$ $159.7,143.7,138.6,138.3,136.9,130.5,129.4,127.8,126.8,125.8,120.6,116.0$, $114.1,111.3,55.3,52.1,51.2,43.4,42.9,20.8$ (see spectrum $) .[\alpha]_{\mathrm{D}}^{25}=+5(c=1$, $\mathrm{CHCl}_{3}$ ). IR (film) $v_{\max } / \mathrm{cm}^{-1}: 2963,2922,2905,2867,1600,1583,1489,1458,1256$, 1155, 1050, 991, 913, 750. HRMS (ESI): Calcd. for $\mathrm{C}_{22} \mathrm{H}_{25} \mathrm{O}\left([\mathrm{M}+\mathrm{H}]^{+}\right) \mathrm{m} / \mathrm{z} 305.1900$, found $\mathrm{m} / \mathrm{z} 305.1896$.

HPLC conditions: Chiral column IB, $n$-hexane $/ i-\mathrm{PrOH}=99: 1$, flow rate $=0.3 \mathrm{~mL} / \mathrm{min}$, wavelength $=210 \mathrm{~nm}, t_{\mathrm{R}}=13.6 \mathrm{~min}$ for major isomer, $t_{\mathrm{R}}=14.4 \mathrm{~min}$ for minor isomer (see spectrum). 


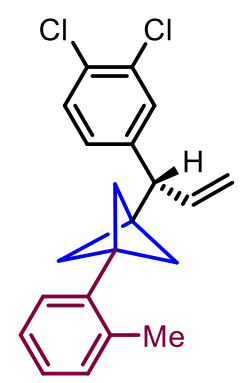

According to general procedure A: $o$-tolylmagnesium bromide ( $2 \mathrm{M}$ in $\mathrm{Et}_{2} \mathrm{O}, 0.64$ mmol), [1.1.1]propellane (1.28 mmol) and tert-butyl (1-(3,4-dichlorophenyl)allyl) carbonate $(0.40 \mathrm{mmol})$ provided the product as a colorless oil $(90.0 \mathrm{mg}, 69 \%$ yield, $>99 \%$ ee, pentane as an eluent).

${ }^{1} \mathrm{H}$ NMR (400 MHz, $\left.\mathrm{CDCl}_{3}\right) \delta 7.43(\mathrm{~d}, J=8.2 \mathrm{~Hz}, 1 \mathrm{H}), 7.32(\mathrm{~d}, J=2.0 \mathrm{~Hz}, 1 \mathrm{H}), 7.18$ $-7.12(\mathrm{~m}, 2 \mathrm{H}), 7.12-7.08(\mathrm{~m}, 2 \mathrm{H}), 7.06(\mathrm{dd}, J=8.2,2.0 \mathrm{~Hz}, 1 \mathrm{H}), 6.09$ (ddd, $J=$ $17.1,10.2,8.2 \mathrm{~Hz}, 1 \mathrm{H}), 5.22(\mathrm{ddd}, J=10.2,1.6,0.9 \mathrm{~Hz}, 1 \mathrm{H}), 5.14(\mathrm{dt}, J=17.0,1.4$ $\mathrm{Hz}, 1 \mathrm{H}), 3.50(\mathrm{~d}, J=8.2 \mathrm{~Hz}, 1 \mathrm{H}), 2.38(\mathrm{~s}, 3 \mathrm{H}), 2.10-1.83(\mathrm{~m}, 6 \mathrm{H})$ (see spectrum) . ${ }^{13} \mathrm{C} \mathrm{NMR}\left(100 \mathrm{MHz}, \mathrm{CDCl}_{3}\right) \delta 142.4,138.2,137.2,136.9,132.4,130.6,130.4,130.3$, 130.0, 127.8, 127.5, 127.0, 125.8, 116.9, 51.1, 51.0, 43.6, 42.6, 20.8 (see spectrum). $[\alpha]_{\mathrm{D}}^{25}=+15\left(c=1, \mathrm{CHCl}_{3}\right)$. IR (film) $v_{\max } / \mathrm{cm}^{-1}: 2965,2928,2906,2868,1488,1471$, 1390, 1255, 1132, 1030, 919, 809, 747. HRMS (EI): Calcd. for $\mathrm{C}_{21} \mathrm{H}_{20} \mathrm{Cl}_{2}\left(\left[\mathrm{M}-\mathrm{CH}_{3}\right]^{+}\right)$ $\mathrm{m} / \mathrm{z} 327.0702$, found $\mathrm{m} / \mathrm{z} 327.0700$.

HPLC conditions: Chiral column IB, $n$-hexane, flow rate $=0.8 \mathrm{~mL} / \mathrm{min}$, wavelength $=$ $210 \mathrm{~nm}, t_{\mathrm{R}}=11.1 \mathrm{~min}$ for major isomer, $t_{\mathrm{R}}=11.7 \mathrm{~min}$ for minor isomer (see spectrum).

\section{(S)-1-(1-(naphthalen-2-yl)allyl)-3-(o-tolyl)bicyclo[1.1.1]pentane (12)}

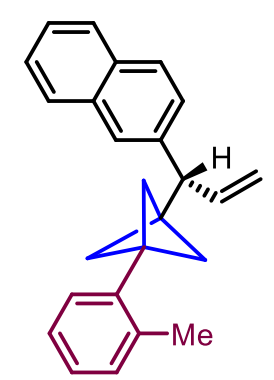

According to general procedure A: $o$-tolylmagnesium bromide ( $2 \mathrm{M}$ in $\mathrm{Et}_{2} \mathrm{O}, 0.64$ $\mathrm{mmol})$, [1.1.1]propellane $(1.28 \mathrm{mmol})$ and tert-butyl (1-(naphthalen-2-yl)allyl) carbonate $(0.40 \mathrm{mmol})$ provided the product as a colorless oil $(94.6 \mathrm{mg}, 73 \%$ yield, $>99 \%$ ee, pentane as an eluent).

${ }^{1} \mathrm{H}$ NMR $\left(400 \mathrm{MHz}, \mathrm{CDCl}_{3}\right) \delta 7.94-7.81(\mathrm{~m}, 3 \mathrm{H}), 7.69-7.62(\mathrm{~m}, 1 \mathrm{H}), 7.54-7.42$ 
(m, 2H), $7.38(\mathrm{dd}, J=8.5,1.8 \mathrm{~Hz}, 1 \mathrm{H}), 7.20-7.01(\mathrm{~m}, 4 \mathrm{H}), 6.27$ (ddd, $J=17.0,10.3$, $8.2 \mathrm{~Hz}, 1 \mathrm{H}), 5.32-5.10(\mathrm{~m}, 2 \mathrm{H}), 3.70(\mathrm{~d}, J=8.2 \mathrm{~Hz}, 1 \mathrm{H}), 2.35(\mathrm{~s}, 3 \mathrm{H}), 2.11-1.96$ $(\mathrm{m}, 6 \mathrm{H})$ (see spectrum $) .{ }^{13} \mathrm{C} \mathrm{NMR}\left(100 \mathrm{MHz}, \mathrm{CDCl}_{3}\right) \delta 139.6,138.5,138.3,136.8$, $133.7,132.3,130.4,127.8,127.7,127.6,126.7,126.3,125.9,125.7,125.3,116.2$, 52.0, 51.2, 43.5, 42.9, 20.7 (two signals are missing due to overlap) (see spectrum). $[\alpha]_{\mathrm{D}}{ }^{25}=+34\left(c=1, \mathrm{CHCl}_{3}\right)$. IR (film) $v_{\max } / \mathrm{cm}^{-1}: 2964,2868,1256,812,746$. HRMS (APCI): Calcd. for $\mathrm{C}_{25} \mathrm{H}_{25} \mathrm{O}\left([\mathrm{M}+\mathrm{H}]^{+}\right) \mathrm{m} / \mathrm{z} 325.1951$, found $\mathrm{m} / \mathrm{z} 325.1951$.

HPLC conditions: Chiral column IB, $n$-hexane, flow rate $=1.0 \mathrm{~mL} / \mathrm{min}$, wavelength $=$ $210 \mathrm{~nm}, t_{\mathrm{R}}=14.0 \mathrm{~min}$ for minor isomer, $t_{\mathrm{R}}=18.5 \mathrm{~min}$ for major isomer ( $\underline{\text { see }}$ spectrum).

(S)-1-(1-(2-Bromo-4-fluorophenyl)allyl)-3-(o-tolyl)bicyclo[1.1.1]pentane (13)

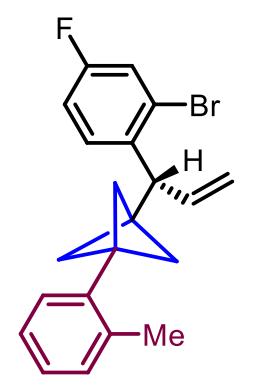

According to general procedure $\mathrm{A}$ : $o$-tolylmagnesium bromide $\left(2 \mathrm{M}\right.$ in $\mathrm{Et}_{2} \mathrm{O}, 0.64$ $\mathrm{mmol})$, [1.1.1]propellane $(1.28 \mathrm{mmol})$ and 1-(2-bromo-4-fluorophenyl)allyl tert-butyl carbonate $(0.40 \mathrm{mmol})$ provided the product as a colorless oil $(80.7 \mathrm{mg}, 57 \%$ yield, pentane as an eluent).

${ }^{1} \mathrm{H}$ NMR (400 MHz, $\left.\mathrm{CDCl}_{3}\right) \delta 7.55(\mathrm{dd}, J=8.8,5.5 \mathrm{~Hz}, 1 \mathrm{H}), 7.15-7.10(\mathrm{~m}, 2 \mathrm{H})$, $7.10-7.05(\mathrm{~m}, 2 \mathrm{H}), 6.99(\mathrm{dd}, J=9.9,3.1 \mathrm{~Hz}, 1 \mathrm{H}), 6.84(\mathrm{ddd}, J=8.8,7.7,3.0 \mathrm{~Hz}$, $1 \mathrm{H}), 6.05$ (ddd, $J=17.0,10.3,8.0 \mathrm{~Hz}, 1 \mathrm{H}), 5.24-5.15$ (m, 2H), 4.18 (dq, $J=7.9,1.2$ $\mathrm{Hz}, 1 \mathrm{H}), 2.36(\mathrm{~s}, 3 \mathrm{H}), 2.12-1.99$ (m, 6H) (see spectrum) ${ }^{19} \mathrm{~F}$ NMR (377 MHz, $\left.\mathrm{CDCl}_{3}\right) \delta-114.5(\mathrm{~m})$ (see spectrum) ${ }^{13} \mathrm{C} \mathrm{NMR}\left(100 \mathrm{MHz}, \mathrm{CDCl}_{3}\right) \delta 162.0(\mathrm{~d}, J=$ $246.3 \mathrm{~Hz}), 143.5(\mathrm{~d}, J=6.9 \mathrm{~Hz}), 138.3,136.9,134.1(\mathrm{~d}, J=7.9 \mathrm{~Hz}), 130.6,127.8$, 127.0, 125.8, $118.8(\mathrm{~d}, J=3.0 \mathrm{~Hz}), 117.4,116.1(\mathrm{~d}, J=23.1 \mathrm{~Hz}), 115.0(\mathrm{~d}, J=22.6$ $\mathrm{Hz}), 51.3,49.6(\mathrm{~d}, J=1.5 \mathrm{~Hz}), 43.7,42.4,20.9$ (see spectrum) $.[\alpha]_{\mathrm{D}}^{25}=+34(c=1$, $\mathrm{CHCl}_{3}$ ). IR (film) $v_{\text {max }} / \mathrm{cm}^{-1}: 2965,2924,2908,2869,1602,1575,1466,1273,1256$, 1162, 1027, 920, 808, 752. HRMS (EI): Calcd. for $\mathrm{C}_{21} \mathrm{H}_{20} \mathrm{BrF}\left(\left[\mathrm{M}-\mathrm{CH}_{3}\right]^{+}\right) \mathrm{m} / \mathrm{z}$ 355.0492 , found $\mathrm{m} / \mathrm{z} 355.0489$.

The ee of product $\mathbf{1 3}$ could not be determined by HPLC, therefore, the ee was determined after hydroboration/oxidation to alcohol 13a. 


\section{1-yl)propan-1-ol (13a)}

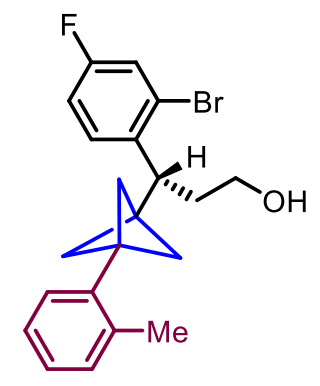

According to general procedure $\mathrm{B}$, product $\mathbf{1 3}$ gave the corresponding alcohol (50.0 $\mathrm{mg}, 78 \%$ yield, $87 \%$ ee, pentane/ $\mathrm{Et}_{2} \mathrm{O}(2 / 1)$ as an eluent).

${ }^{1} \mathrm{H}$ NMR $\left(400 \mathrm{MHz}, \mathrm{CDCl}_{3}\right) \delta 7.55(\mathrm{dd}, J=8.8,5.5 \mathrm{~Hz}, 1 \mathrm{H}), 7.14-7.09(\mathrm{~m}, 2 \mathrm{H})$, $7.08-7.02(\mathrm{~m}, 2 \mathrm{H}), 6.92(\mathrm{dd}, J=9.9,3.1 \mathrm{~Hz}, 1 \mathrm{H}), 6.84(\mathrm{ddd}, J=8.8,7.7,3.1 \mathrm{~Hz}$, 1H), 3.66 (ddd, $J=10.9,4.5,1.8 \mathrm{~Hz}, 1 \mathrm{H}), 3.59$ (ddd, $J=10.7,7.4,4.9 \mathrm{~Hz}, 1 \mathrm{H}$ ), 3.49 (ddd, $J=10.7,8.0,6.6 \mathrm{~Hz}, 1 \mathrm{H}), 2.35(\mathrm{~s}, 3 \mathrm{H}), 2.16-2.07(\mathrm{~m}, 1 \mathrm{H}), 2.05-1.94(\mathrm{~m}$, $6 \mathrm{H}), 1.91-1.82(\mathrm{~m}, 1 \mathrm{H}), 1.37$ (br. s, 1H) (see spectrum) ${ }^{19} \mathrm{~F}$ NMR $\left(377 \mathrm{MHz}, \mathrm{CDCl}_{3}\right)$ $\delta-114.2(\mathrm{~m})$ (see spectrum) ${ }^{13} \mathrm{C} \mathrm{NMR}\left(100 \mathrm{MHz}, \mathrm{CDCl}_{3}\right) \delta 162.2(\mathrm{~d}, J=246.5 \mathrm{~Hz})$, $143.6(\mathrm{~d}, J=6.7 \mathrm{~Hz}), 138.2,136.9,134.1(\mathrm{~d}, J=8.0 \mathrm{~Hz}), 130.6,127.8,127.0,125.8$, $119.8(\mathrm{~d}, J=2.5 \mathrm{~Hz}), 115.2(\mathrm{~d}, J=22.5 \mathrm{~Hz}), 115.1(\mathrm{~d}, J=22.6 \mathrm{~Hz}), 60.9,51.0,43.0$, $42.9,41.3,34.8,20.8$ (see spectrum $) .[\alpha]_{\mathrm{D}}{ }^{25}=+57\left(c=1, \mathrm{CHCl}_{3}\right)$. IR (film) $v_{\max } / \mathrm{cm}^{-1}$ : 3363, 2962, 2926, 2868, 1600, 1578, 1466, 1267, 1160, 1053, 1026, 810, 747. HRMS (ESI): Calcd. for $\mathrm{C}_{21} \mathrm{H}_{22} \mathrm{BrFNaO}\left([\mathrm{M}+\mathrm{Na}]^{+}\right) \mathrm{m} / \mathrm{z}$ 411.0730, found $\mathrm{m} / \mathrm{z} 411.0731$.

HPLC conditions: Chiral column IA, $n$-hexane $/ i-\mathrm{PrOH}=98: 2$, flow rate $=0.5$ $\mathrm{mL} / \mathrm{min}$, wavelength $=210 \mathrm{~nm}, t_{\mathrm{R}}=37.2 \mathrm{~min}$ for major isomer, $t_{\mathrm{R}}=39.4 \mathrm{~min}$ for minor isomer (see spectrum).

(S)-2-Chloro-3-(1-(3-(o-tolyl)bicyclo[1.1.1]pentan-1-yl)allyl)quinoline (14)

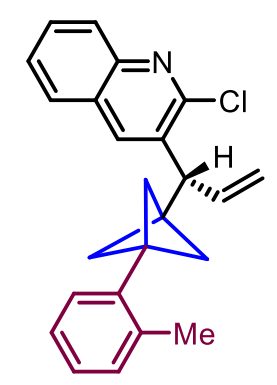

According to general procedure A: $o$-tolylmagnesium bromide ( $2 \mathrm{M}$ in $\mathrm{Et}_{2} \mathrm{O}, 0.64$ $\mathrm{mmol})$, [1.1.1]propellane (1.28 $\mathrm{mmol})$ and tert-butyl (1-(2-chloroquinolin-3-yl)allyl) carbonate $(0.40 \mathrm{mmol})$ provided the product as a colorless oil $(102.0 \mathrm{mg}, 71 \%$ yield, $88 \%$ ee, pentane/ $/ \mathrm{Et}_{2} \mathrm{O}(4 / 1)$ as an eluent). 
${ }^{1} \mathrm{H}$ NMR $\left(400 \mathrm{MHz}, \mathrm{CDCl}_{3}\right) \delta 8.08-8.01(\mathrm{~m}, 2 \mathrm{H}), 7.83(\mathrm{~d}, J=8.2 \mathrm{~Hz}, 1 \mathrm{H}), 7.72$ (ddd, $J=8.4,6.9,1.4 \mathrm{~Hz}, 1 \mathrm{H}), 7.57$ (ddd, $J=8.0,6.9,1.1 \mathrm{~Hz}, 1 \mathrm{H}), 7.16-7.02(\mathrm{~m}$, $4 \mathrm{H}), 6.28-6.15(\mathrm{~m}, 1 \mathrm{H}), 5.31-5.20(\mathrm{~m}, 2 \mathrm{H}), 4.33(\mathrm{~d}, J=8.1 \mathrm{~Hz}, 1 \mathrm{H}), 2.34(\mathrm{~s}, 3 \mathrm{H})$, $2.14-2.02(\mathrm{~m}, 6 \mathrm{H})$ (see spectrum) ${ }^{13} \mathrm{C} \mathrm{NMR}\left(100 \mathrm{MHz}, \mathrm{CDCl}_{3}\right) \delta 151.5,146.3$, 138.0, 136.79, 136.76, 136.6, 134.1, 130.5, 130.0, 128.3, 127.7, 127.4, 127.3, 127.0, $126.9,125.7,117.6,51.1,46.6,43.6,42.5,20.7$ (see spectrum $) .[\alpha]_{\mathrm{D}}^{25}=-20(c=1$, $\mathrm{CHCl}_{3}$ ). IR (film) $v_{\max } / \mathrm{cm}^{-1}: 3018,2971,1739,1366,1216,746,668$. HRMS (APCI): Calcd. for $\mathrm{C}_{24} \mathrm{H}_{23} \mathrm{NCl}\left([\mathrm{M}+\mathrm{H}]^{+}\right) \mathrm{m} / \mathrm{z} 360.1514$, found $\mathrm{m} / \mathrm{z} 360.1512$.

HPLC conditions: Chiral column IA, $n$-hexane $/ i-\mathrm{PrOH}=98 / 2$, flow rate $=1.0$ $\mathrm{mL} / \mathrm{min}$, wavelength $=210 \mathrm{~nm}, t_{\mathrm{R}}=6.1 \mathrm{~min}$ for minor isomer, $t_{\mathrm{R}}=11.9 \mathrm{~min}$ for major isomer (see spectrum).

\section{(S)-3-(1-(3-(o-Tolyl)bicyclo[1.1.1]pentan-1-yl)allyl)quinoline (15)}

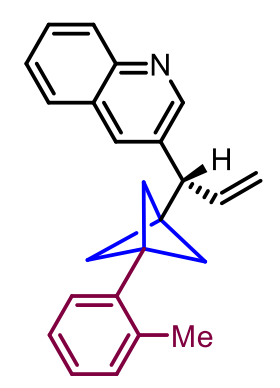

According to general procedure A: $o$-tolylmagnesium bromide $\left(2 \mathrm{M}\right.$ in $\mathrm{Et}_{2} \mathrm{O}, 0.64$ mmol), [1.1.1]propellane $(1.28 \mathrm{mmol})$ and tert-butyl (1-(quinolin-3-yl)allyl) carbonate $(0.40 \mathrm{mmol})$ provided the product as a colorless oil $(87.1 \mathrm{mg}, 67 \%$ yield, $98 \%$ ee, pentane/ $\mathrm{Et}_{2} \mathrm{O}(4 / 1)$ as an eluent).

${ }^{1} \mathrm{H} \mathrm{NMR}\left(400 \mathrm{MHz}, \mathrm{CDCl}_{3}\right) \delta 8.84(\mathrm{~d}, J=2.2 \mathrm{~Hz}, 1 \mathrm{H}), 8.13(\mathrm{~d}, J=8.4 \mathrm{~Hz}, 1 \mathrm{H}), 7.96$ $(\mathrm{d}, J=2.2 \mathrm{~Hz}, 1 \mathrm{H}), 7.83(\mathrm{dd}, J=8.2,1.4 \mathrm{~Hz}, 1 \mathrm{H}), 7.70(\mathrm{ddd}, J=8.4,6.9,1.5 \mathrm{~Hz}, 1 \mathrm{H})$, 7.56 (ddd, $J=8.1,6.9,1.2 \mathrm{~Hz}, 1 \mathrm{H}), 7.16-7.04$ (m, 4H), 6.26 (ddd, $J=17.1,10.3,8.1$ $\mathrm{Hz}, 1 \mathrm{H}), 5.27$ (dt, $J=10.3,1.2 \mathrm{~Hz}, 1 \mathrm{H}), 5.19$ (dt, $J=17.1,1.3 \mathrm{~Hz}, 1 \mathrm{H}), 3.76(\mathrm{~d}, J=$ $8.0 \mathrm{~Hz}, 1 \mathrm{H}), 2.34(\mathrm{~s}, 3 \mathrm{H}), 2.11-2.01(\mathrm{~m}, 6 \mathrm{H})$ (see spectrum $).{ }^{13} \mathrm{C}$ NMR $(100 \mathrm{MHz}$, $\left.\mathrm{CDCl}_{3}\right) \delta 151.5,147.1,138.1,137.1,136.8,134.8,134.0,130.5,129.3,128.9,128.2$, 127.7, 127.6, 126.9, 126.7, 125.7, 117.1, 51.1, 49.4, 43.8, 42.8, 20.7 (see spectrum). $[\alpha]_{\mathrm{D}}{ }^{25}=+29\left(c=1, \mathrm{CHCl}_{3}\right)$. IR (film) $v_{\max } / \mathrm{cm}^{-1}: 2970,2868,1739,1375,1217,751$. HRMS (APCI): Calcd. for $\mathrm{C}_{24} \mathrm{H}_{24} \mathrm{~N}\left([\mathrm{M}+\mathrm{H}]^{+}\right) \mathrm{m} / \mathrm{z} 326.1903$, found $\mathrm{m} / \mathrm{z}$ 326.1903.

HPLC conditions: Chiral column IA, $n$-hexane $/ i-\mathrm{PrOH}=95 / 1$, flow rate $=1.0$ $\mathrm{mL} / \mathrm{min}$, wavelength $=210 \mathrm{~nm}, t_{\mathrm{R}}=18.6 \mathrm{~min}$ for minor isomer, $t_{\mathrm{R}}=22.1 \mathrm{~min}$ for major isomer (see spectrum). 


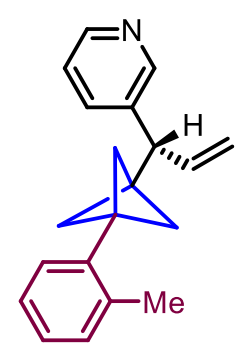

According to general procedure A: $o$-tolylmagnesium bromide $\left(2 \mathrm{M}\right.$ in $\mathrm{Et}_{2} \mathrm{O}, 0.64$ $\mathrm{mmol})$, [1.1.1]propellane $(1.28 \mathrm{mmol})$ and tert-butyl (1-(pyridin-3-yl)allyl) carbonate $(0.40 \mathrm{mmol})$ provided the product as a colorless oil $(45.1 \mathrm{mg}, 41 \%$ yield, $97 \%$ ee, pentane/ $\mathrm{Et}_{2} \mathrm{O}(4 / 1)$ as an eluent).

${ }^{1} \mathrm{H}$ NMR $\left(400 \mathrm{MHz}, \mathrm{CDCl}_{3}\right) \delta 8.41(\mathrm{dd}, J=4.8,1.7 \mathrm{~Hz}, 1 \mathrm{H}), 8.39(\mathrm{~d}, J=2.0 \mathrm{~Hz}, 1 \mathrm{H})$, $7.44(\mathrm{dt}, J=7.8,2.0 \mathrm{~Hz}, 1 \mathrm{H}), 7.23-7.15(\mathrm{~m}, 1 \mathrm{H}), 7.07-6.93(\mathrm{~m}, 4 \mathrm{H}), 6.04$ (ddd, $J=$ $17.1,10.3,8.1 \mathrm{~Hz}, 1 \mathrm{H}), 5.12(\mathrm{ddd}, J=10.3,1.6,1.0 \mathrm{~Hz}, 1 \mathrm{H}), 5.04(\mathrm{dt}, J=17.1,1.4$ $\mathrm{Hz}, 1 \mathrm{H}), 3.46(\mathrm{~d}, J=8.1 \mathrm{~Hz}, 1 \mathrm{H}), 2.26(\mathrm{~s}, 3 \mathrm{H}), 1.97-1.86(\mathrm{~m}, 6 \mathrm{H})$ (see spectrum). ${ }^{13} \mathrm{C}$ NMR $\left(100 \mathrm{MHz}, \mathrm{CDCl}_{3}\right) \delta 149.7,147.8,138.1,137.3,137.1,136.8,135.3,130.5$, $127.6,126.9,125.7,123.3,116.8,50.9,49.2,43.6,42.6,20.7$ (see spectrum) $) \cdot[\alpha]_{\mathrm{D}}{ }^{25}=$ $+28\left(c=0.5, \mathrm{CHCl}_{3}\right)$. IR (film) $v_{\max } / \mathrm{cm}^{-1}: 2969,2869,1739,1217,751$. HRMS (APCI): Calcd. for $\mathrm{C}_{20} \mathrm{H}_{22} \mathrm{~N}\left([\mathrm{M}+\mathrm{H}]^{+}\right) \mathrm{m} / \mathrm{z} 276.1747$, found $\mathrm{m} / \mathrm{z} 276.1748$.

HPLC conditions: Chiral column IA, $n$-hexane $/ i-\mathrm{PrOH}=97 / 3$, flow rate $=1.0$ $\mathrm{mL} / \mathrm{min}$, wavelength $=210 \mathrm{~nm}, t_{\mathrm{R}}=10.2 \mathrm{~min}$ for minor isomer, $t_{\mathrm{R}}=10.9 \min$ for major isomer (see spectrum).

\section{(S)-1-(7-Phenylhept-1-en-4-yn-3-yl)-3-(o-tolyl)bicyclo[1.1.1]pentane (17)}

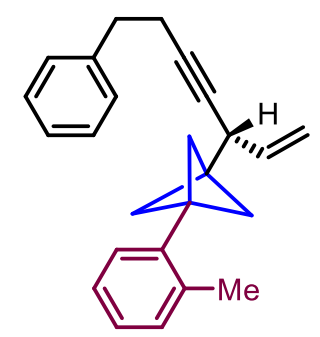

According to general procedure A: $o$-tolylmagnesium bromide $\left(2 \mathrm{M}\right.$ in $\mathrm{Et}_{2} \mathrm{O}, 0.64$ mmol), [1.1.1]propellane (1.28 mmol) and tert-butyl (7-phenylhept-1-en-4-yn-3-yl) carbonate $(0.40 \mathrm{mmol})$ provided the product as a colorless oil $(95.5 \mathrm{mg}, 77 \%$ yield, $94 \%$ ee, pentane as an eluent).

${ }^{1} \mathrm{H}$ NMR $\left(400 \mathrm{MHz}, \mathrm{CDCl}_{3}\right) \delta 7.23-7.08(\mathrm{~m}, 5 \mathrm{H}), 7.08-6.96(\mathrm{~m}, 4 \mathrm{H}), 5.67$ (ddd, $J$ $=17.0,10.0,6.3 \mathrm{~Hz}, 1 \mathrm{H}), 5.19(\mathrm{dt}, J=17.0,1.6 \mathrm{~Hz}, 1 \mathrm{H}), 5.03(\mathrm{ddd}, J=9.9,1.3 \mathrm{~Hz}$, 1H), $3.19-3.08(\mathrm{~m}, 1 \mathrm{H}), 2.77(\mathrm{t}, J=7.4 \mathrm{~Hz}, 2 \mathrm{H}), 2.47(\mathrm{td}, J=7.4,2.1 \mathrm{~Hz}, 2 \mathrm{H}), 2.29$ 
(s, 3H), 1.95 - $1.84(\mathrm{~m}, 6 \mathrm{H})$ (see spectrum). $\left.{ }^{13} \mathrm{C} \mathrm{NMR} \mathrm{(100} \mathrm{MHz,} \mathrm{CDCl}_{3}\right) \delta 140.9$, 138.4, 136.9, 135.4, 130.5, 128.6, 128.4, 127.8, 126.8, 126.2, 125.7, 115.7, 83.6, 78.6, 50.6, 42.8, 40.9, 38.0, 35.6, 21.0, 20.8 (see spectrum) $) .[\alpha]_{\mathrm{D}}{ }^{25}=+63\left(c=1, \mathrm{CHCl}_{3}\right) . \mathrm{IR}$ (film) $v_{\max } / \mathrm{cm}^{-1}: 2971,1739,1365,1216,746$. HRMS (EI): Calcd. for $\mathrm{C}_{24} \mathrm{H}_{23}$ ([M$\left.\mathrm{CH}_{3}\right]^{+}$) $\mathrm{m} / \mathrm{z} 311.1794$, found $\mathrm{m} / \mathrm{z} 311.1793$.

HPLC conditions: Chiral column IB, $n$-hexane, flow rate $=1.0 \mathrm{~mL} / \mathrm{min}$, wavelength $=$ $210 \mathrm{~nm}, t_{\mathrm{R}}=18.3 \mathrm{~min}$ for major isomer, $t_{\mathrm{R}}=21.5 \mathrm{~min}$ for minor isomer (see spectrum).

1-((S)-1-((S)-4-(Prop-1-en-2-yl)cyclohex-1-en-1-yl)allyl)-3(o-tolyl)bicyclo[1.1.1]pentane (18)

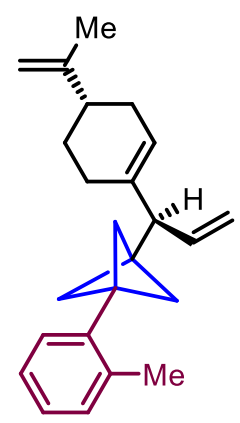

According to general procedure A: $o$-tolylmagnesium bromide $\left(2 \mathrm{M}\right.$ in $\mathrm{Et}_{2} \mathrm{O}, 0.64$ mmol), [1.1.1]propellane $(1.28 \mathrm{mmol}),(R)$-phosphoramidite ligand $(R)-\mathbf{L}(0.032$ mmol), and tert-butyl (1-((S)-4-(prop-1-en-2-yl)cyclohex-1-en-1-yl)allyl) carbonate $(0.40 \mathrm{mmol})$ provided the product as a colorless oil $(76.3 \mathrm{mg}, 60 \%$ yield, >95:5 $\mathrm{dr}$, pentane as an eluent). The dr was determined by GC/MS analysis.

When the same reaction was performed with the $(S)$-phosphoramidite ligand $(S)$-L, the product was formed in $69 \%$ yield with $75: 25 \mathrm{dr}$, still favoring the same $(S, S)$-diastereomer.

${ }^{1} \mathrm{H}$ NMR (400 MHz, $\mathrm{CDCl}_{3}$ ) Major isomer: $\delta 7.16-7.05(\mathrm{~m}, 4 \mathrm{H}), 5.90(\mathrm{ddd}, J=16.5$, 10.7, 8.5 Hz, 1H), $5.52-5.47(\mathrm{~m}, 1 \mathrm{H}), 5.08-5.00(\mathrm{~m}, 2 \mathrm{H}), 4.75-4.70(\mathrm{~m}, 2 \mathrm{H}), 2.80$ $(\mathrm{d}, J=8.6 \mathrm{~Hz}, 1 \mathrm{H}), 2.39(\mathrm{~s}, 3 \mathrm{H}), 2.22-2.07(\mathrm{~m}, 4 \mathrm{H}), 2.05-1.95(\mathrm{~m}, 7 \mathrm{H}), 1.86-$ $1.78(\mathrm{~m}, 1 \mathrm{H}), 1.75(\mathrm{t}, J=1.1 \mathrm{~Hz}, 3 \mathrm{H}), 1.52-1.41(\mathrm{~m}, 1 \mathrm{H})$ (see spectrum). Minor isomer: $\delta 7.13-7.05(\mathrm{~m}, 4 \mathrm{H}), 6.67(\mathrm{dt}, J=16.8,10.5 \mathrm{~Hz}, 1 \mathrm{H}), 5.88-5.83(\mathrm{~m}, 1 \mathrm{H})$, 5.16 (ddt, $J=16.8,2.2,0.7 \mathrm{~Hz}, 1 \mathrm{H}), 5.02$ (ddt, $J=10.2,2.2,0.8 \mathrm{~Hz}, 1 \mathrm{H}$ ), $4.72-4.69$ (m, 2H), $2.80(\mathrm{dt}, J=14.2,3.4 \mathrm{~Hz}, 1 \mathrm{H}), 2.46(\mathrm{~d}, J=5.8 \mathrm{~Hz}, 1 \mathrm{H}), 2.38(\mathrm{~s}, 3 \mathrm{H}), 2.37-$ $2.31(\mathrm{~m}, 1 \mathrm{H}), 2.10-2.04(\mathrm{~m}, 6 \mathrm{H}), 1.98-1.92(\mathrm{~m}, 1 \mathrm{H}), 1.90-1.83(\mathrm{~m}, 1 \mathrm{H}), 1.73(\mathrm{t}, J$ $=1.2 \mathrm{~Hz}, 3 \mathrm{H}), 1.51(\mathrm{td}, J=13.0,5.8 \mathrm{~Hz}, 1 \mathrm{H}), 1.30-1.18(\mathrm{~m}, 1 \mathrm{H})($ see spectrum $) .{ }^{13} \mathrm{C}$ NMR (100 MHz, $\mathrm{CDCl}_{3}$ ) Major isomer: $\delta$ 150.1, 138.7, 138.1, 138.0, 136.9, 130.4, 127.7, 126.7, 125.7, 121.9, 114.9, 108.5, 52.8, 51.5, 42.9, 41.9, 41.3, 30.9, 28.1, 28.0, 
20.8, 20.7 (see spectrum). Minor isomer: $\delta$ 150.2, 142.6, 138.5, 136.9, 132.5, 130.4, 127.7, 126.7, 125.7, 125.2, 115.2, 108.5, 52.9, 45.8, 43.1, 41.5, 40.7, 34.7, 32.0, 26.4, 20.8, 20.7 (see spectrum $) .[\alpha]_{\mathrm{D}}{ }^{25}=-44\left(c=1, \mathrm{CHCl}_{3}\right)$. IR (film) $v_{\max } / \mathrm{cm}^{-1}: 2970,1738$, 1371, 1217, 749. HRMS (APCI): Calcd. for $\mathrm{C}_{24} \mathrm{H}_{31}\left([\mathrm{M}+\mathrm{H}]^{+}\right) \mathrm{m} / \mathrm{z} 319.2420$, found $\mathrm{m} / \mathrm{z} 319.2423$.

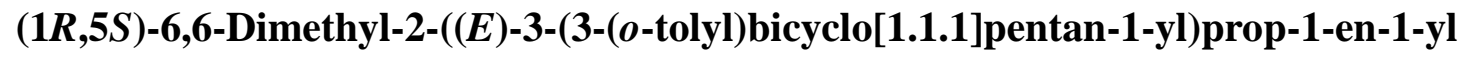
)bicyclo[3.1.1]hept-2-ene (19)

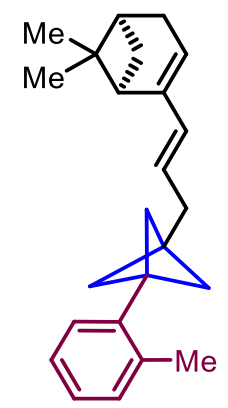

According to general procedure $\mathrm{A}$ : $o$-tolylmagnesium bromide $\left(2 \mathrm{M}\right.$ in $\mathrm{Et}_{2} \mathrm{O}, 0.64$ mmol $)$ [1.1.1]propellane $(1.28 \quad \mathrm{mmol})$ and tert-butyl (1-((1R,5S)-6,6-dimethylbicyclo[3.1.1]hept-2-en-2-yl)allyl) carbonate $(0.40 \mathrm{mmol})$ provided the product as a colorless oil $(20.4 \mathrm{mg}, 16 \%$ yield with $>95: 5$ selectiviely for the linear regioisomer, pentane as an eluent). The branched/linear ratio was determined by ${ }^{1} \mathrm{H}$ NMR analysis.

${ }^{1} \mathrm{H}$ NMR (400 MHz, $\left.\mathrm{CDCl}_{3}\right) \delta 7.20-7.02(\mathrm{~m}, 4 \mathrm{H}), 6.08(\mathrm{~d}, J=15.5 \mathrm{~Hz}, 1 \mathrm{H}), 5.59-$ $5.38(\mathrm{~m}, 2 \mathrm{H}), 2.56(\mathrm{td}, J=5.7,1.4 \mathrm{~Hz}, 1 \mathrm{H}), 2.47-2.28(\mathrm{~m}, 8 \mathrm{H}), 2.16-2.10(\mathrm{~m}, 1 \mathrm{H})$, $2.01(\mathrm{~s}, 6 \mathrm{H}), 1.35(\mathrm{~s}, 3 \mathrm{H}), 1.18(\mathrm{~d}, J=8.7 \mathrm{~Hz}, 1 \mathrm{H}), 0.82(\mathrm{~s}, 3 \mathrm{H})$ (see spectrum) ${ }^{13} \mathrm{C}$ NMR $\left(100 \mathrm{MHz}, \mathrm{CDCl}_{3}\right) \delta 146.5,138.9,137.0,132.9,130.5,127.9,126.8,125.8$, 123.0, 122.0, 52.3, 43.5, 41.5, 41.2, 39.6, 37.9, 35.8, 32.0, 31.5, 26.6, 20.9, 20.8 (see

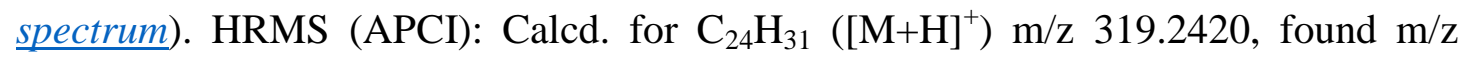
319.2415 . 


\section{IIIb. Grignard Reagent Scope}

\section{(S)-1-(1-(Naphthalen-2-yl)allyl)-3-phenylbicyclo[1.1.1]pentane (20)}

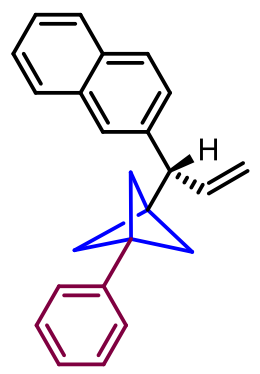

According to general procedure $\mathrm{A}$ : phenylmagnesium bromide ( $3 \mathrm{M}$ in $\mathrm{Et}_{2} \mathrm{O}, 0.64$ mmol), [1.1.1]propellane $(1.28 \mathrm{mmol})$ and tert-butyl (1-(naphthalen-2-yl)allyl) carbonate $(0.40 \mathrm{mmol})$ provided the product as a white solid $(99.2 \mathrm{mg}, 80 \%$ yield, $99 \%$ ee, pentane as an eluent).

${ }^{1} \mathrm{H}$ NMR $\left(400 \mathrm{MHz}, \mathrm{CDCl}_{3}\right) \delta 7.90-7.78(\mathrm{~m}, 3 \mathrm{H}), 7.67(\mathrm{~s}, 1 \mathrm{H}), 7.52-7.44(\mathrm{~m}, 2 \mathrm{H})$, $7.39(\mathrm{dd}, J=8.5,1.8 \mathrm{~Hz}, 1 \mathrm{H}), 7.32-7.27(\mathrm{~m}, 2 \mathrm{H}), 7.25-7.16(\mathrm{~m}, 3 \mathrm{H}), 6.27$ (ddd, $J$ $=17.1,10.3,8.2 \mathrm{~Hz}, 1 \mathrm{H}), 5.25-5.15(\mathrm{~m}, 2 \mathrm{H}), 3.71(\mathrm{~d}, J=8.2 \mathrm{~Hz}, 1 \mathrm{H}), 2.04-1.88$ $(\mathrm{m}, 6 \mathrm{H})$ (see spectrum) ${ }^{13} \mathrm{C} \mathrm{NMR}\left(100 \mathrm{MHz}, \mathrm{CDCl}_{3}\right) \delta 141.3,139.7,138.4,133.8$, $132.4,128.2$, 127.9, 127.8, 127.8, 126.9, 126.4, 126.4, 126.1, 126.0, 125.5, 116.3, 52.0, 51.3, 42.6, 41.9 (see spectrum $) .[\alpha]_{\mathrm{D}}^{25}=+30\left(c=1, \mathrm{CHCl}_{3}\right)$. IR (film) $v_{\max } / \mathrm{cm}^{-1}$ : 3057, 2964, 2924, 2906, 2867, 1633, 1601, 1508, 1445, 1273, 1255, 1156, 1068, 989, 915, 866, 811, 776, 745. HRMS (APCI): Calcd. for $\mathrm{C}_{24} \mathrm{H}_{22}\left([\mathrm{M}+\mathrm{H}]^{+}\right) \mathrm{m} / \mathrm{z} 311.1794$, found $\mathrm{m} / \mathrm{z} 311.1791$.

HPLC conditions: Chiral column IB, $n$-hexane, flow rate $=1.0 \mathrm{~mL} / \mathrm{min}$, wavelength $=$ $210 \mathrm{~nm}, t_{\mathrm{R}}=13.9 \mathrm{~min}$ for minor isomer, $t_{\mathrm{R}}=19.6 \mathrm{~min}$ for major isomer (see spectrum).

(S)-1-(1-(Naphthalen-2-yl)allyl)-3-(p-tolyl)bicyclo[1.1.1]pentane (21)

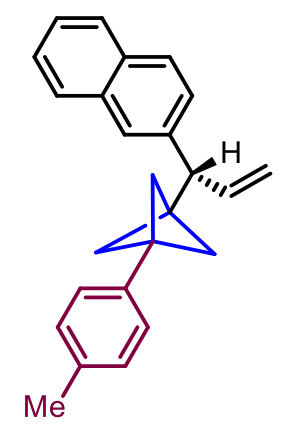

According to general procedure $\mathrm{A}$ : p-tolylmagnesium bromide $\left(0.5 \mathrm{M}\right.$ in $\mathrm{Et}_{2} \mathrm{O}, 0.64$ mmol), [1.1.1]propellane (1.28 mmol) and tert-butyl (1-(naphthalen-2-yl)allyl) carbonate $(0.40 \mathrm{mmol})$ provided the product as a colorless oil $(98.8 \mathrm{mg}, 76 \%$ 
yield, $>99 \%$ ee, pentane as an eluent).

${ }^{1} \mathrm{H}$ NMR $\left(500 \mathrm{MHz}, \mathrm{CDCl}_{3}\right) \delta 7.85-7.79(\mathrm{~m}, 3 \mathrm{H}), 7.64(\mathrm{~s}, 1 \mathrm{H}), 7.49-7.43(\mathrm{~m}, 2 \mathrm{H})$, $7.36(\mathrm{dd}, J=8.5,1.8 \mathrm{~Hz}, 1 \mathrm{H}), 7.11-7.04(\mathrm{~m}, 4 \mathrm{H}), 6.24(\mathrm{ddd}, J=17.0,10.3,8.2 \mathrm{~Hz}$, $1 \mathrm{H}), 5.21-5.12(\mathrm{~m}, 2 \mathrm{H}), 3.67(\mathrm{~d}, J=8.1 \mathrm{~Hz}, 1 \mathrm{H}), 2.31(\mathrm{~s}, 3 \mathrm{H}), 2.01-1.82(\mathrm{~m}, 6 \mathrm{H})$ ${ }^{13} \mathrm{C}$ NMR $\left(125 \mathrm{MHz}, \mathrm{CDCl}_{3}\right) \delta 139.7,138.3,138.3,135.9,133.6,132.3,128.8$, $127.74,127.69,127.6,126.8,126.3,125.92,125.87,125.3,116.1,51.90,51.2,42.3$, 41.7, 21.1. (see spectrum) $.[\alpha]_{\mathrm{D}}^{25}=+41\left(c=1, \mathrm{CHCl}_{3}\right)$. IR (film) $v_{\max } / \mathrm{cm}^{-1}: 3050$, 2963, 2918, 2907, 2867, 1633, 1600, 1522, 1508, 1444, 1255, 1153, 1018, 914, 856, 815, 794, 774, 748. HRMS (APCI): Calcd. for $\mathrm{C}_{25} \mathrm{H}_{24}\left([\mathrm{M}+\mathrm{H}]^{+}\right) \mathrm{m} / \mathrm{z} 325.1951$, found $\mathrm{m} / \mathrm{z} 325.1950$.

HPLC conditions: Chiral column IB, $n$-hexane $/ i$-PrOH $=100: 0$, flow rate $=1.0$ $\mathrm{mL} / \mathrm{min}$, wavelength $=210 \mathrm{~nm}, t_{\mathrm{R}}=13.9 \mathrm{~min}$ for minor isomer, $t_{\mathrm{R}}=20.1 \mathrm{~min}$ for major isomer (see spectrum).

(S)-1-(4-(tert-Butyl)phenyl)-3-(1-(naphthalen-2-yl)allyl)bicyclo[1.1.1]pentane (22)

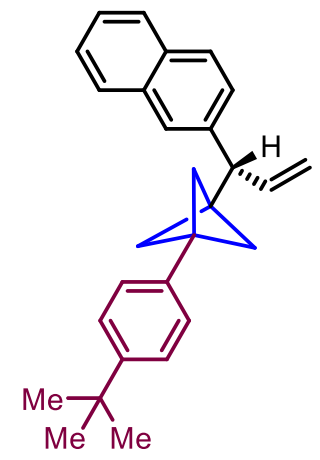

According to general procedure A: (4-(tert-butyl)phenyl)magnesium bromide (2.0 M in $\left.\mathrm{Et}_{2} \mathrm{O}, \quad 0.64 \mathrm{mmol}\right), \quad[1.1 .1]$ propellane $(1.28 \mathrm{mmol})$ and tert-butyl (1-(naphthalen-2-yl)allyl) carbonate $(0.40 \mathrm{mmol})$ provided the product as a white solid (92.2 mg, 63\% yield, $98 \%$ ee, pentane as an eluent).

${ }^{1} \mathrm{H}$ NMR $\left(400 \mathrm{MHz}, \mathrm{CDCl}_{3}\right) \delta 7.88-7.80(\mathrm{~m}, 3 \mathrm{H}), 7.65(\mathrm{~s}, 1 \mathrm{H}), 7.51-7.43(\mathrm{~m}, 2 \mathrm{H})$, $7.38(\mathrm{dd}, J=8.5,1.7 \mathrm{~Hz}, 1 \mathrm{H}), 7.35-7.30(\mathrm{~m}, 2 \mathrm{H}), 7.18-7.11(\mathrm{~m}, 2 \mathrm{H}), 6.26(\mathrm{ddd}, J$ $=17.0,10.3,8.2 \mathrm{~Hz}, 1 \mathrm{H}), 5.25-5.11(\mathrm{~m}, 2 \mathrm{H}), 3.69(\mathrm{~d}, J=8.1 \mathrm{~Hz}, 1 \mathrm{H}), 2.00-1.87$ $(\mathrm{m}, 6 \mathrm{H}), 1.32(\mathrm{~s}, 9 \mathrm{H})$ (see spectrum) ${ }^{13} \mathrm{C} \mathrm{NMR}\left(100 \mathrm{MHz}, \mathrm{CDCl}_{3}\right) \delta 149.3,139.8$, $138.5,138.4,133.8,132.4,127.9,127.8,127.8,126.9,126.4,126.0,125.9,125.4$, $125.1,116.2,52.1,51.3,42.4,41.9,34.6,31.5$ (see spectrum) $) \cdot[\alpha]_{\mathrm{D}}^{25}=+33(c=1$, $\mathrm{CHCl}_{3}$ ). IR (film) $v_{\text {max }} / \mathrm{cm}^{-1}: 3055,2962,2905,2867,1629,1602,1508,1461,1363$, 1269, 1254, 1158, 1019, 915, 856, 842, 815, 801, 777, 749. HRMS (APCI): Calcd. for $\mathrm{C}_{28} \mathrm{H}_{30}\left([\mathrm{M}+\mathrm{H}]^{+}\right) \mathrm{m} / \mathrm{z} 367.2420$, found $\mathrm{m} / \mathrm{z}$ 367.2418.

HPLC conditions: Chiral column IB, $n$-hexane, flow rate $=1.0 \mathrm{~mL} / \mathrm{min}$, wavelength $=$ 
$210 \mathrm{~nm}, t_{\mathrm{R}}=16.9 \mathrm{~min}$ for major isomer, $t_{\mathrm{R}}=10.9 \mathrm{~min}$ for minor isomer (see spectrum).

(S)-1-(4-Methoxyphenyl)-3-(1-(naphthalen-2-yl)allyl)bicyclo[1.1.1]pentane (23)

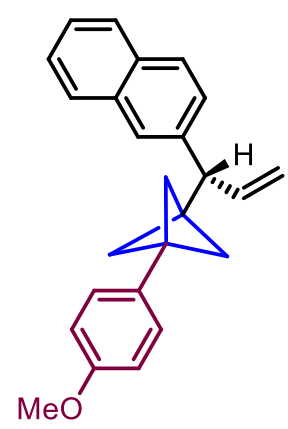

According to general procedure A: (4-methoxyphenyl)magnesium bromide $(0.5 \mathrm{M}$ in THF, $0.64 \mathrm{mmol}$ ) [THF was removed under high vacuum prior to addition of a diethyl ether solution of 1], [1.1.1]propellane $(1.2 \mathrm{mmol})$ and tert-butyl (1-(naphthalen-2-yl)allyl) carbonate $(0.40 \mathrm{mmol})$ provided the product with minor impurities. HRMS (APCI): Calcd. for $\mathrm{C}_{25} \mathrm{H}_{24} \mathrm{O}\left([\mathrm{M}+\mathrm{H}]^{+}\right) \mathrm{m} / \mathrm{z} 341.1900$, found $\mathrm{m} / \mathrm{z}$ 341.1899.

Due to the presence of minor impurities in product $\mathbf{2 3}$, the isolated yield was determined after hydroboration/oxidation to alcohol 23a.

\section{(S)-3-(3-(4-Methoxyphenyl)bicyclo[1.1.1]pentan-1-yl)-3-}

(naphthalen-2-yl)propan-1-ol (23a)

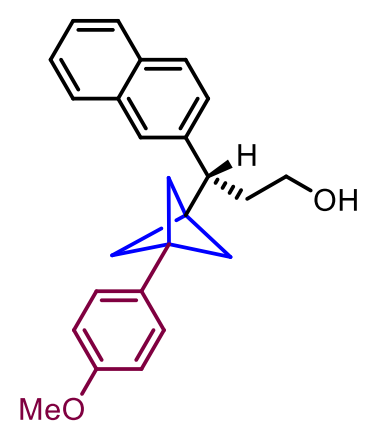

According to general procedure $\mathrm{B}$, product $\mathbf{2 3}$ gave the corresponding alcohol (91.2mg, 60\% yield, $98 \%$ ee, pentane/ $\mathrm{Et}_{2} \mathrm{O}$ (2/1) as an eluent) (over 2 steps).

${ }^{1} \mathrm{H}$ NMR (400 MHz, $\left.\mathrm{CDCl}_{3}\right) \delta 7.97-7.74(\mathrm{~m}, 3 \mathrm{H}), 7.61(\mathrm{~s}, 1 \mathrm{H}), 7.55-7.42(\mathrm{~m}, 2 \mathrm{H})$, $7.33(\mathrm{dd}, J=8.4,1.8 \mathrm{~Hz}, 1 \mathrm{H}), 7.17-7.01(\mathrm{~m}, 2 \mathrm{H}), 6.88-6.75(\mathrm{~m}, 2 \mathrm{H}), 3.77(\mathrm{~s}, 3 \mathrm{H})$, $3.62(\mathrm{ddd}, J=10.3,7.1,5.0 \mathrm{~Hz}, 1 \mathrm{H}), 3.52(\mathrm{ddd}, J=10.5,7.8,6.7 \mathrm{~Hz}, 1 \mathrm{H}), 3.11(\mathrm{dd}, J$ $=10.8,4.6 \mathrm{~Hz}, 1 \mathrm{H}), 2.21-2.01(\mathrm{~m}, 2 \mathrm{H}), 1.94-1.70(\mathrm{~m}, 6 \mathrm{H})$ (see spectrum $).{ }^{13} \mathrm{C}$ NMR $\left(100 \mathrm{MHz}, \mathrm{CDCl}_{3}\right) \delta 158.3,139.7,133.7,132.5,128.0,127.8,127.7,127.2$, 
$126.7,126.5,126.0,125.4,113.7,61.5,55.4,51.0,43.7,42.5,41.5,34.5$ (one signal is missing due to overlap) (see spectrum) $.[\alpha]_{\mathrm{D}}^{25}=+30\left(c=1, \mathrm{CHCl}_{3}\right)$. IR (film) $v_{\max } / \mathrm{cm}^{-1}: 3327,2958,2904,2865,1610,1520,1504,1463,1295,1246,1173,1036$, 837, 817, 793, 751. HRMS (ESI): Calcd. for $\mathrm{C}_{25} \mathrm{H}_{26} \mathrm{NaO}_{2}\left([\mathrm{M}+\mathrm{Na}]^{+}\right) \mathrm{m} / \mathrm{z} 381.1825$, found $\mathrm{m} / \mathrm{z} 381.1824$.

HPLC conditions: Chiral column IA, $n$-hexane $/ i-\mathrm{PrOH}=95: 5$, flow rate $=1.0$ $\mathrm{mL} / \mathrm{min}$, wavelength $=210 \mathrm{~nm}, t_{\mathrm{R}}=18.9 \mathrm{~min}$ for major isomer, $t_{\mathrm{R}}=24.2 \mathrm{~min}$ for minor isomer (see spectrum).

(S)-1-(2,4-Dimethylphenyl)-3-(1-(naphthalen-2-yl)allyl)bicyclo[1.1.1]pentane (24)

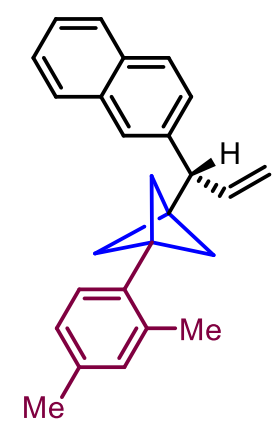

According to general procedure A: (2,4-dimethylphenyl)magnesium bromide $(0.5 \mathrm{M}$ in THF, $0.64 \mathrm{mmol}$ ) [THF was removed under high vacuum prior to addition of a diethyl ether solution of 1], [1.1.1]propellane $(1.28 \mathrm{mmol})$ and tert-butyl (1-(naphthalen-2-yl)allyl) carbonate $(0.40 \mathrm{mmol})$ provided the product as a colorless oil ( $83.8 \mathrm{mg}, 62 \%$ yield, $>99 \%$ ee, pentane as an eluent).

${ }^{1} \mathrm{H}$ NMR (400 MHz, $\left.\mathrm{CDCl}_{3}\right) \delta 7.92-7.78(\mathrm{~m}, 3 \mathrm{H}), 7.67(\mathrm{~s}, 1 \mathrm{H}), 7.55-7.44(\mathrm{~m}, 2 \mathrm{H})$, $7.39(\mathrm{dd}, J=8.5,1.8 \mathrm{~Hz}, 1 \mathrm{H}), 7.04-6.88(\mathrm{~m}, 3 \mathrm{H}), 6.28(\mathrm{ddd}, J=17.0,10.3,8.2 \mathrm{~Hz}$, $1 \mathrm{H}), 5.30-5.09(\mathrm{~m}, 2 \mathrm{H}), 3.70(\mathrm{~d}, J=8.2 \mathrm{~Hz}, 1 \mathrm{H}), 2.33$ (s, 3H), 2.30 (s, 3H), $2.14-$ $1.95(\mathrm{~m}, 6 \mathrm{H})$ (see spectrum) ${ }^{13} \mathrm{C}$ NMR $\left(100 \mathrm{MHz}, \mathrm{CDCl}_{3}\right) \delta 139.8,138.4,136.8$, $136.5,135.7,133.8,132.4,131.4,127.9,127.8,127.8,126.9,126.4,126.0,125.4$, 116.2, 52.1, 51.3, 43.3, 43.0, 21.0, 20.7 (two signals are missing due to overlap) (see spectrum $) .[\alpha]_{\mathrm{D}}^{25}=+36\left(c=1, \mathrm{CHCl}_{3}\right)$. IR $($ film $) v_{\max } / \mathrm{cm}^{-1}: 3056,2964,2923,2906$, 2868, 1633, 1599, 1508, 1451, 1373, 1272, 1256, 1157, 990, 915, 856, 815, 798, 776, 749. HRMS (APCI): Calcd. for $\mathrm{C}_{26} \mathrm{H}_{26}\left([\mathrm{M}+\mathrm{H}]^{+}\right) \mathrm{m} / \mathrm{z} 339.2107$, found $\mathrm{m} / \mathrm{z} 339.2105$.

HPLC conditions: Chiral column IB, $n$-hexane, flow rate $=1.0 \mathrm{~mL} / \mathrm{min}$, wavelength $=$ $210 \mathrm{~nm}, t_{\mathrm{R}}=14.5 \mathrm{~min}$ for minor isomer, $t_{\mathrm{R}}=17.9 \mathrm{~min}$ for major isomer (see spectrum). 


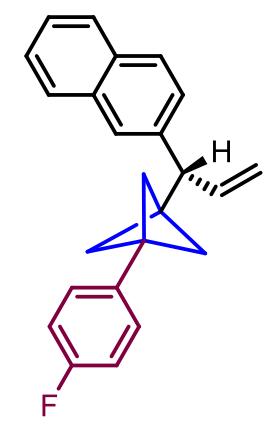

According to general procedure A: 4-fluorophenylmagnesium bromide (2.0 $\mathrm{M}_{\text {in }} \mathrm{Et}_{2} \mathrm{O}$, $0.64 \mathrm{mmol})$, [1.1.1]propellane $(1.28 \mathrm{mmol})$ and tert-butyl (1-(naphthalen-2-yl)allyl) carbonate $(0.40 \mathrm{mmol})$ provided the product as a white solid $(66.9 \mathrm{mg}, 51 \%$ yield, 99\% ee, pentane as an eluent).

${ }^{1} \mathrm{H}$ NMR $\left(400 \mathrm{MHz}, \mathrm{CDCl}_{3}\right) \delta 7.89-7.80(\mathrm{~m}, 3 \mathrm{H}), 7.67-7.64(\mathrm{~m}, 1 \mathrm{H}), 7.54-7.44$ (m, 2H), $7.37(\mathrm{dd}, J=8.5,1.8 \mathrm{~Hz}, 1 \mathrm{H}), 7.17-7.09(\mathrm{~m}, 2 \mathrm{H}), 7.01-6.92(\mathrm{~m}, 2 \mathrm{H}), 6.26$ $(\mathrm{ddd}, J=17.0,10.3,8.1 \mathrm{~Hz}, 1 \mathrm{H}), 5.24-5.15(\mathrm{~m}, 2 \mathrm{H}), 3.70(\mathrm{~d}, J=8.1 \mathrm{~Hz}, 1 \mathrm{H}), 1.98-$ $1.86(\mathrm{~m}, 6 \mathrm{H})$ (see spectrum) ${ }^{13} \mathrm{C} \mathrm{NMR}\left(100 \mathrm{MHz}, \mathrm{CDCl}_{3}\right) \delta 161.6(\mathrm{~d}, J=242.5 \mathrm{~Hz})$, 139.5, 138.1, 137.0 (d, $J=3.2 \mathrm{~Hz}), 133.6,132.3,127.8,127.7,127.6,127.5$ (d, $J=$ $7.9 \mathrm{~Hz}), 126.7,126.3,125.9,125.3,116.2,114.8(\mathrm{~d}, J=21.0 \mathrm{~Hz}), 51.8,51.2,42.0$, 41.7 (see spectrum) $.[\alpha]_{\mathrm{D}}^{25}=+35\left(c=1, \mathrm{CHCl}_{3}\right)$. IR (film) $v_{\max } / \mathrm{cm}^{-1}: 2966,2869$, 1518, 1220, 811. HRMS (EI): Calcd. for $\mathrm{C}_{24} \mathrm{H}_{21} \mathrm{~F}\left([\mathrm{M}]^{+}\right) \mathrm{m} / \mathrm{z} 328.1622$, found $\mathrm{m} / \mathrm{z}$ 328.1623 .

HPLC conditions: Chiral column IB, $n$-hexane, flow rate $=1.0 \mathrm{~mL} / \mathrm{min}$, wavelength $=$ $210 \mathrm{~nm}, t_{\mathrm{R}}=14.9 \mathrm{~min}$ for minor isomer, $t_{\mathrm{R}}=22.3 \mathrm{~min}$ for major isomer (see spectrum).

(S)-1-(4-Chlorophenyl)-3-(1-(naphthalen-2-yl)allyl)bicyclo[1.1.1]pentane (26)

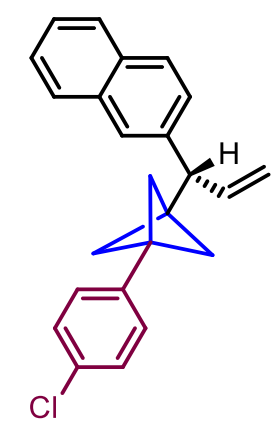

According to general procedure A: (4-chlorophenyl)magnesium bromide (1.0 $\mathrm{M}$ in $\left.\mathrm{Et}_{2} \mathrm{O}, \quad 0.64 \mathrm{mmol}\right), \quad[1.1 .1]$ propellane $(1.28 \mathrm{mmol})$ and tert-butyl (1-(naphthalen-2-yl)allyl) carbonate $(0.40 \mathrm{mmol})$ provided the product as a white 
solid (78.4 mg, 57\% yield, 99\% ee, pentane as an eluent).

${ }^{1} \mathrm{H}$ NMR (400 MHz, $\left.\mathrm{CDCl}_{3}\right) \delta 7.96-7.74(\mathrm{~m}, 3 \mathrm{H}), 7.65(\mathrm{~s}, 1 \mathrm{H}), 7.54-7.43(\mathrm{~m}, 2 \mathrm{H})$, $7.37(\mathrm{dd}, J=8.5,1.8 \mathrm{~Hz}, 1 \mathrm{H}), 7.27-7.22(\mathrm{~m}, 2 \mathrm{H}), 7.15-7.02(\mathrm{~m}, 2 \mathrm{H}), 6.25$ (ddd, $J$ $=17.0,10.3,8.2 \mathrm{~Hz}, 1 \mathrm{H}), 5.42-5.03(\mathrm{~m}, 2 \mathrm{H}), 3.69(\mathrm{~d}, J=8.1 \mathrm{~Hz}, 1 \mathrm{H}), 2.04-1.81$ $(\mathrm{m}, 6 \mathrm{H})$ (see spectrum $) .{ }^{13} \mathrm{C} \mathrm{NMR}\left(100 \mathrm{MHz}, \mathrm{CDCl}_{3}\right) \delta 139.8,139.6,138.2,133.8$, $132.4,132.2$, 128.3, 127.9, 127.8, 127.8, 127.6, 126.8, 126.4, 126.0, 125.5, 116.4, $51.9,51.3,42.1,41.9$ (see spectrum $) .[\alpha]_{\mathrm{D}}{ }^{25}=+30\left(c=1, \mathrm{CHCl}_{3}\right)$. IR (film) $v_{\max } / \mathrm{cm}^{-1}$ : 3053, 2964, 2930, 2907, 2868, 1638, 1599, 1509, 1489, 1254, 1155, 1089, 1015, 916 , 857, 840, 816, 798, 774, 749, 725. HRMS (APCI): Calcd. for $\mathrm{C}_{24} \mathrm{H}_{21} \mathrm{Cl}\left([\mathrm{M}+\mathrm{H}]^{+}\right) \mathrm{m} / \mathrm{z}$ 345.1405 , found $\mathrm{m} / \mathrm{z} 345.1403$.

HPLC conditions: Chiral column IB, $n$-hexane, flow rate $=1.0 \mathrm{~mL} / \mathrm{min}$, wavelength $=$ $210 \mathrm{~nm}, t_{\mathrm{R}}=30.9 \mathrm{~min}$ for major isomer, $t_{\mathrm{R}}=19.0 \mathrm{~min}$ for minor isomer (see spectrum).

(S)-1-(Naphthalen-2-yl)-3-(1-(naphthalen-2-yl)allyl)bicyclo[1.1.1]pentane (27)

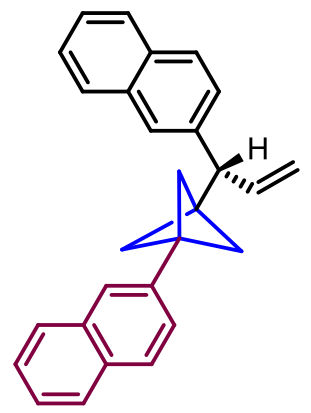

According to general procedure A: naphthalen-2-ylmagnesium bromide $(0.5 \mathrm{M}$ in THF, $0.64 \mathrm{mmol}$ ) [THF was removed under high vacuum prior to addition of a diethyl ether solution of 1], [1.1.1]propellane $(1.28 \mathrm{mmol})$ and tert-butyl (1-(naphthalen-2-yl)allyl) carbonate $(0.40 \mathrm{mmol})$ provided the product as a white solid (89.3 mg, 62\% yield, $98 \%$ ee, pentane as an eluent).

${ }^{1} \mathrm{H}$ NMR (400 MHz, $\left.\mathrm{CDCl}_{3}\right) \delta 7.89-7.81(\mathrm{~m}, 3 \mathrm{H}), 7.81-7.73(\mathrm{~m}, 3 \mathrm{H}), 7.68(\mathrm{~s}, 1 \mathrm{H})$, 7.59 (s, 1H), $7.53-7.37(\mathrm{~m}, 5 \mathrm{H}), 7.34$ (dd, $J=8.4,1.7 \mathrm{~Hz}, 1 \mathrm{H}), 6.29$ (ddd, $J=17.0$, 10.3, 8.1 Hz, 1H), $5.35-5.03(\mathrm{~m}, 2 \mathrm{H}), 3.73(\mathrm{~d}, J=8.2 \mathrm{~Hz}, 1 \mathrm{H}), 2.17-1.83(\mathrm{~m}, 6 \mathrm{H})$ (see spectrum) ${ }^{13} \mathrm{C}$ NMR $\left(100 \mathrm{MHz}, \mathrm{CDCl}_{3}\right) \delta 139.7,138.8,138.4,133.8,133.4$, $132.4,132.3,127.9,127.8,127.8,127.8,127.8,127.7,126.9,126.5,126.1,126.0$, $125.5,124.7,124.5,116.3,52.0,51.3,42.9,42.1$ (one signal is missing due to overlap) (see spectrum) $.[\alpha]_{\mathrm{D}}^{25}=+44\left(c=1, \mathrm{CHCl}_{3}\right)$. IR (film) $v_{\max } / \mathrm{cm}^{-1}: 3054,2964,2921$, 2905, 2867, 1632, 1600, 1508, 1444, 1367, 1255, 1236, 1197, 1151, 986, 915, 891, 857, 819, 802, 774, 744. HRMS (APCI): Calcd. for $\mathrm{C}_{28} \mathrm{H}_{24}\left([\mathrm{M}+\mathrm{H}]^{+}\right) \mathrm{m} / \mathrm{z} 361.1951$, found $\mathrm{m} / \mathrm{z} 361.1949$. 
HPLC conditions: Chiral column IB, $n$-hexane $/ i-\mathrm{PrOH}=99: 1$, flow rate $=0.7 \mathrm{~mL} / \mathrm{min}$, wavelength $=230 \mathrm{~nm}, t_{\mathrm{R}}=7.0 \mathrm{~min}$ for major isomer, $t_{\mathrm{R}}=5.3 \mathrm{~min}$ for minor isomer (see spectrum).

(S)-1-(1-(Naphthalen-2-yl)allyl)-3-(prop-1-en-2-yl)bicyclo[1.1.1]pentane (28)

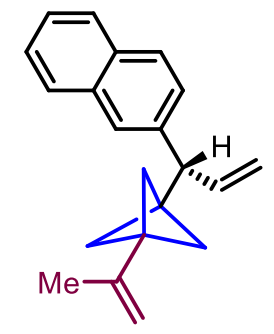

According to general procedure A: prop-1-en-2-ylmagnesium bromide (0.5 M in THF, $0.64 \mathrm{mmol}$ ) [THF was removed under high vacuum prior to addition of a diethyl ether solution of 1], [1.1.1]propellane (1.28 mmol) and tert-butyl (1-(naphthalen-2-yl)allyl) carbonate $(0.40 \mathrm{mmol})$ provided the product as a colorless oil $(57.0 \mathrm{mg}, 52 \%$ yield, $97 \%$ ee, pentane as an eluent).

${ }^{1} \mathrm{H}$ NMR (400 MHz, $\left.\mathrm{CDCl}_{3}\right) \delta 7.85-7.78(\mathrm{~m}, 3 \mathrm{H}), 7.61(\mathrm{~s}, 1 \mathrm{H}), 7.49-7.42(\mathrm{~m}, 2 \mathrm{H})$, $7.33(\mathrm{dd}, J=8.5,1.7 \mathrm{~Hz}, 1 \mathrm{H}), 6.21$ (ddd, $J=17.0,10.3,8.1 \mathrm{~Hz}, 1 \mathrm{H}), 5.20-5.09$ (m, $2 \mathrm{H}), 4.73-4.59(\mathrm{~m}, 2 \mathrm{H}), 3.62(\mathrm{~d}, J=8.2 \mathrm{~Hz}, 1 \mathrm{H}), 1.73-1.61(\mathrm{~m}, 9 \mathrm{H})$ (see spectrum) ${ }^{13} \mathrm{C}$ NMR $\left(100 \mathrm{MHz}, \mathrm{CDCl}_{3}\right) \delta 145.0,139.8,138.5,133.7,132.4,127.8,127.7,126.9$, $126.4,126.0,125.4,116.1,109.7,52.0,49.7,44.4,41.4,19.1$ (one signal is missing due to overlap) (see spectrum) $.[\alpha]_{\mathrm{D}}^{25}=+29\left(c=0.85, \mathrm{CHCl}_{3}\right)$. IR (film) $v_{\max } / \mathrm{cm}^{-1}$ : 3056, 2964, 2908, 2867, 1635, 1599, 1508, 1448, 1375, 1255, 1194, 989, 915, 889, 866, 812, 775, 749. HRMS (APCI): Calcd. for $\mathrm{C}_{21} \mathrm{H}_{22}\left([\mathrm{M}+\mathrm{H}]^{+}\right) \mathrm{m} / \mathrm{z} 275.1794$, found $\mathrm{m} / \mathrm{z} 275.1791$.

HPLC conditions: Chiral column IB, $n$-hexane, flow rate $=1.0 \mathrm{~mL} / \mathrm{min}$, wavelength $=$ $210 \mathrm{~nm}, t_{\mathrm{R}}=11.0 \mathrm{~min}$ for major isomer, $t_{\mathrm{R}}=8.1 \mathrm{~min}$ for minor isomer (see spectrum) .

(S)-1-Allyl-3-(1-(naphthalen-2-yl)allyl)bicyclo[1.1.1]pentane (29)

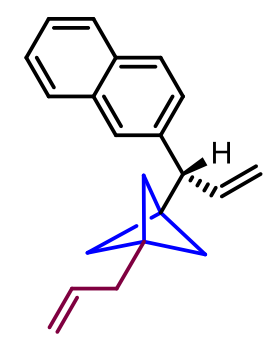

According to general procedure A: allylmagnesium bromide (1 $\mathrm{M}$ in $\mathrm{Et}_{2} \mathrm{O}, 0.64$ 
mmol), [1.1.1]propellane (1.28 mmol) and tert-butyl (1-(naphthalen-2-yl)allyl) carbonate $(0.40 \mathrm{mmol})$ provided the product as a colorless oil $(60.3 \mathrm{mg}, 55 \%$ yield, $98 \%$ ee, pentane as an eluent).

${ }^{1} \mathrm{H}$ NMR (400 MHz, $\left.\mathrm{CDCl}_{3}\right) \delta 7.85-7.76(\mathrm{~m}, 3 \mathrm{H}), 7.61-7.57(\mathrm{~m}, 1 \mathrm{H}), 7.49-7.41$ $(\mathrm{m}, 2 \mathrm{H}), 7.31(\mathrm{dd}, J=8.4,1.8 \mathrm{~Hz}, 1 \mathrm{H}), 6.19(\mathrm{ddd}, J=17.0,10.3,8.1 \mathrm{~Hz}, 1 \mathrm{H}), 5.68$ (ddt, $J=16.8,10.4,7.2 \mathrm{~Hz}, 1 \mathrm{H}$ ), 5.14 (ddd, $J=10.3,1.8,1.0 \mathrm{~Hz}, 1 \mathrm{H}), 5.10$ (ddd, $J=$ 17.0, 1.8, 1.2 Hz, 1H), $5.00-4.91(\mathrm{~m}, 2 \mathrm{H}), 3.58(\mathrm{~d}, J=8.2 \mathrm{~Hz}, 1 \mathrm{H}), 2.20$ (d, $J=7.2$ $\mathrm{Hz}, 2 \mathrm{H}), 1.56-1.45(\mathrm{~m}, 6 \mathrm{H})$ (see spectrum) ${ }^{13} \mathrm{C} \mathrm{NMR}\left(100 \mathrm{MHz}, \mathrm{CDCl}_{3}\right) \delta 139.9$, $138.5,135.7,133.6,132.2,127.7,127.58,127.57,126.8,126.2,125.8,125.2,115.8$, $115.6,52.1,49.1,43.1,39.5,36.9$ (see spectrum $) .[\alpha]_{\mathrm{D}}^{25}=+34\left(c=1, \mathrm{CHCl}_{3}\right) . \mathrm{IR}$ (film) $v_{\max } / \mathrm{cm}^{-1}: 2961,2866,1640,1251,912,746$. HRMS (EI): Calcd. for $\mathrm{C}_{21} \mathrm{H}_{22}$ $\left([\mathrm{M}]^{+}\right) \mathrm{m} / \mathrm{z} 274.1716$, found $\mathrm{m} / \mathrm{z} 274.1720$.

HPLC conditions: Chiral column IB, $n$-hexane, flow rate $=1.0 \mathrm{~mL} / \mathrm{min}$, wavelength $=$ $210 \mathrm{~nm}, t_{\mathrm{R}}=7.0 \mathrm{~min}$ for minor isomer, $t_{\mathrm{R}}=9.7 \mathrm{~min}$ for major isomer (see spectrum) .

\section{(S)-3-(3-Ethylbicyclo[1.1.1]pentan-1-yl)-3-(naphthalen-2-yl)propan-1-ol (30a)}

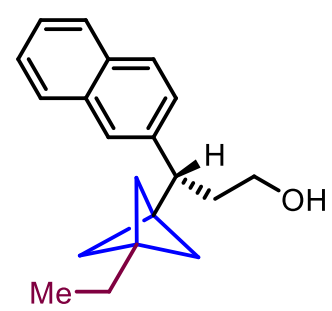

According to general procedure $\mathrm{A}$ : ethylmagnesium bromide $\left(3 \mathrm{M}\right.$ in $\mathrm{Et}_{2} \mathrm{O}, 0.64$ mmol), [1.1.1]propellane $(1.28 \mathrm{mmol})$ and tert-butyl (1-(naphthalen-2-yl)allyl) carbonate $(0.40 \mathrm{mmol})$ provided the product $\mathbf{3 0}$ as a colorless oil with minor side products. The yield was determined after hydroboration/oxidation to give alcohol 30a.

According to general procedure $\mathrm{B}$, product $\mathbf{3 0}$ gave the corresponding alcohol (76.1 $\mathrm{mg}, 63 \%$ yield, $94 \%$ ee, pentane/Et ${ }_{2} \mathrm{O}(2 / 1)$ as an eluent) (over 2 steps).

${ }^{1} \mathrm{H}$ NMR $\left(400 \mathrm{MHz}, \mathrm{CDCl}_{3}\right) \delta 7.84-7.74(\mathrm{~m}, 3 \mathrm{H}), 7.55(\mathrm{~d}, J=1.7 \mathrm{~Hz}, 1 \mathrm{H}), 7.48-$ $7.39(\mathrm{~m}, 2 \mathrm{H}), 7.27(\mathrm{dd}, J=8.4,1.7 \mathrm{~Hz}, 1 \mathrm{H}), 3.63-3.44(\mathrm{~m}, 2 \mathrm{H}), 2.99(\mathrm{dd}, J=10.8$, $4.6 \mathrm{~Hz}, 1 \mathrm{H}), 2.12-1.93(\mathrm{~m}, 2 \mathrm{H}), 1.46-1.32(\mathrm{~m}, 8 \mathrm{H}), 1.15$ (br. s, $1 \mathrm{H}), 0.77$ (t, $J=7.5$ $\mathrm{Hz}, 3 \mathrm{H})$ (see spectrum) ${ }^{13} \mathrm{C} \mathrm{NMR}\left(100 \mathrm{MHz}, \mathrm{CDCl}_{3}\right) \delta 139.9,133.5,132.3,127.7$, 127.59, 127.56, 126.5, 126.4, 125.8, 125.2, 61.5, 48.2, 43.9, 42.8, 40.7, 34.5, 24.8, 10.5 (see spectrum $) .[\alpha]_{\mathrm{D}}^{25}=+8\left(c=1, \mathrm{CHCl}_{3}\right)$. IR (film) $v_{\max } / \mathrm{cm}^{-1}: 2957,2864,1738$, 1371, 1217. HRMS (ESI): Calcd. for $\mathrm{C}_{20} \mathrm{H}_{24} \mathrm{ONa}\left([\mathrm{M}+\mathrm{Na}]^{+}\right) \mathrm{m} / \mathrm{z} 303.1719$, found $\mathrm{m} / \mathrm{z}$ 303.1713 .

HPLC conditions: Chiral column IB, $n$-hexane $/ i-\mathrm{PrOH}=95: 5$, flow rate $=1.0 \mathrm{~mL} / \mathrm{min}$, 
wavelength $=210 \mathrm{~nm}, t_{\mathrm{R}}=10.3 \mathrm{~min}$ for major isomer, $t_{\mathrm{R}}=13.8 \mathrm{~min}$ for minor isomer (see spectrum).

\section{(S)-3-(3-Cyclopropylbicyclo[1.1.1]pentan-1-yl)-3-(naphthalen-2-yl)propan-1-ol}

(31a)

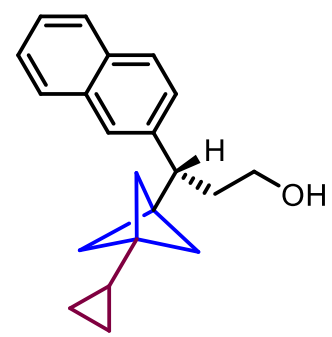

According to general procedure A: cyclopropylmagnesium bromide ( $0.5 \mathrm{M}$ in THF, $0.64 \mathrm{mmol}$ ) [THF was removed under high vacuum prior to addition of a diethyl ether solution of 1], [1.1.1]propellane (1.28 mmol) and tert-butyl (1-(naphthalen-2-yl)allyl) carbonate $(0.40 \mathrm{mmol})$ provided the product as a colorless oil with minor side products. The yield was determined after hydroboration/oxidation to give alcohol 31a.

According to general procedure $\mathrm{B}$, product $\mathbf{3 1}$ gave the corresponding alcohol (57.7 $\mathrm{mg}, 46 \%$ yield, $99 \%$ ee, pentane (2/1) as an eluent) (over 2 steps).

${ }^{1} \mathrm{H}$ NMR (400 MHz, $\left.\mathrm{CDCl}_{3}\right) \delta 7.84-7.72(\mathrm{~m}, 3 \mathrm{H}), 7.53(\mathrm{~d}, J=1.7 \mathrm{~Hz}, 1 \mathrm{H}), 7.48-$ $7.40(\mathrm{~m}, 2 \mathrm{H}), 7.26(\mathrm{dd}, J=8.5,1.7 \mathrm{~Hz}, 1 \mathrm{H}), 3.63-3.39(\mathrm{~m}, 2 \mathrm{H}), 2.98(\mathrm{dd}, J=10.7$, $4.7 \mathrm{~Hz}, 1 \mathrm{H}), 2.11-1.88(\mathrm{~m}, 2 \mathrm{H}), 1.43-1.28(\mathrm{~m}, 6 \mathrm{H}), 1.17$ (br. s, 1H), $0.86-0.72(\mathrm{~m}$, $1 \mathrm{H}), 0.34-0.24(\mathrm{~m}, 2 \mathrm{H}), 0.06--0.02(\mathrm{~m}, 2 \mathrm{H})$ (see spectrum) $){ }^{13} \mathrm{C}$ NMR $(100 \mathrm{MHz}$, $\left.\mathrm{CDCl}_{3}\right) \delta 139.8,133.5,132.3,127.7,127.59,127.56,126.43,126.42,125.8,125.2$, $61.4,48.0,43.7,42.4,41.0,34.4,11.4,2.2$ (see spectrum) $.[\alpha]_{\mathrm{D}}^{25}=+18(c=0.5$, $\mathrm{CHCl}_{3}$ ). IR (film) $v_{\max } / \mathrm{cm}^{-1}: 2971,1738,1355,1217$. HRMS (ESI): Calcd. for $\mathrm{C}_{21} \mathrm{H}_{24} \mathrm{ONa}\left([\mathrm{M}+\mathrm{Na}]^{+}\right) \mathrm{m} / \mathrm{z} 315.1719$, found $\mathrm{m} / \mathrm{z} 315.1718$.

HPLC conditions: Chiral column IB, $n$-hexane $/ i-\mathrm{PrOH}=95: 5$, flow rate $=1.0 \mathrm{~mL} / \mathrm{min}$, wavelength $=210 \mathrm{~nm}, t_{\mathrm{R}}=11.0 \mathrm{~min}$ for major isomer, $t_{\mathrm{R}}=14.3 \mathrm{~min}$ for minor isomer (see spectrum). 


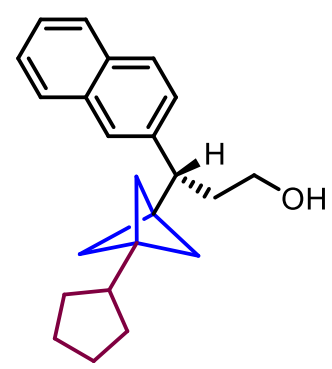

According to general procedure A: cyclopentylmagnesium bromide ( $2 \mathrm{M}$ in $\mathrm{Et}_{2} \mathrm{O}$, $0.64 \mathrm{mmol}),[1.1 .1]$ propellane $(1.28 \mathrm{mmol})$ and tert-butyl (1-(naphthalen-2-yl)allyl) carbonate $(0.40 \mathrm{mmol})$ provided the product as a colorless oil with minor side products. The yield was determined after hydroboration/oxidation to give alcohol 32a.

According to general procedure B, product 32 gave the corresponding alcohol (40.1 $\mathrm{mg}, 74 \%$ yield, $95 \%$ ee, pentane (2/1) as an eluent) (over 2 steps).

${ }^{1} \mathrm{H}$ NMR (400 MHz, $\left.\mathrm{CDCl}_{3}\right) \delta 7.85-7.74(\mathrm{~m}, 3 \mathrm{H}), 7.55(\mathrm{~d}, J=1.7 \mathrm{~Hz}, 1 \mathrm{H}), 7.50-$ $7.40(\mathrm{~m}, 2 \mathrm{H}), 7.28(\mathrm{dd}, J=8.5,1.7 \mathrm{~Hz}, 1 \mathrm{H}), 3.63-3.44(\mathrm{~m}, 2 \mathrm{H}), 3.00(\mathrm{dd}, J=10.7$, $4.7 \mathrm{~Hz}, 1 \mathrm{H}), 2.11-1.94(\mathrm{~m}, 2 \mathrm{H}), 1.92-1.82(\mathrm{~m}, 1 \mathrm{H}), 1.59-1.44(\mathrm{~m}, 6 \mathrm{H}), 1.43-$ $1.33(\mathrm{~m}, 6 \mathrm{H}), 1.28$ (br. s, $1 \mathrm{H}), 1.23-1.10(\mathrm{~m}, 2 \mathrm{H})$ (see spectrum). ${ }^{13} \mathrm{C}$ NMR $(100$ $\left.\mathrm{MHz}, \mathrm{CDCl}_{3}\right) \delta 139.9,133.6,132.3,127.67,127.61,127.58,126.52,126.4,125.8$, $125.2,61.5,47.2,43.9,42.9,42.5,40.4,34.4,29.1,25.7$ (see spectrum) $.[\alpha]_{\mathrm{D}}^{25}=+9(c$ $=1, \mathrm{CHCl}_{3}$ ). IR (film) $v_{\max } / \mathrm{cm}^{-1}: 2951,1739,1366,1217$. HRMS (ESI): Calcd. for $\mathrm{C}_{23} \mathrm{H}_{28} \mathrm{ONa}\left([\mathrm{M}+\mathrm{Na}]^{+}\right) \mathrm{m} / \mathrm{z} 343.2032$, found $\mathrm{m} / \mathrm{z} 343.2025$.

HPLC conditions: Chiral column IB, $n$-hexane $/ i$-PrOH $=95: 5$, flow rate $=1.0 \mathrm{~mL} / \mathrm{min}$, wavelength $=210 \mathrm{~nm}, t_{\mathrm{R}}=11.0 \mathrm{~min}$ for major isomer, $t_{\mathrm{R}}=14.6 \mathrm{~min}$ for minor isomer (see spectrum). 


\section{IIIc. Product Derivatizations}

\section{(S)-4,4,5,5-Tetramethyl-2-(3-phenyl-3-(3-(o-tolyl)bicyclo[1.1.1]pentan-}

\section{1-yl)propyl)-1,3,2-dioxaborolane (34)}
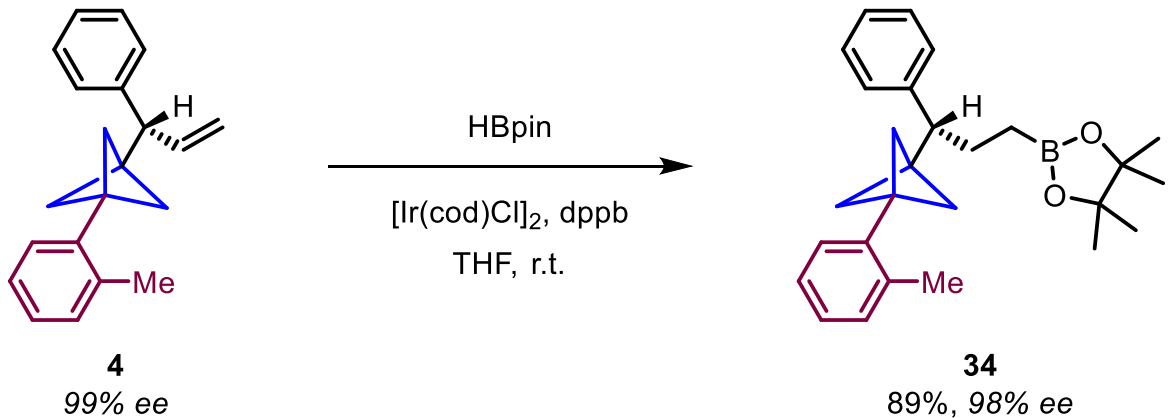

In a glovebox, a flame-dried round bottom flask was charged with $[\operatorname{Ir}(\operatorname{cod}) \mathrm{Cl}]_{2}(3.0$ mol\%) and 1,4-bis(diphenylphosphino)butane (dppb, $5.0 \mathrm{~mol} \%$ ). The solids were dissolved in anhydrous THF $(1.0 \mathrm{~mL})$ and allowed to stir for 20 minutes. A solution of olefin $4(0.15 \mathrm{mmol})$ in THF $(1.0 \mathrm{~mL})$ was added, followed by the dropwise addition of pinacolborane (HBpin, $0.225 \mathrm{mmol}$ ). The flask was sealed with a septum, brought out of the glove box, and the reaction was stirred at r.t. overnight. The solvent was removed under reduced pressure and the product was purified by flash column chromatography with pentane/ $\mathrm{Et}_{2} \mathrm{O}$ (100/0 to 20/1) as eluent to give $\mathbf{3 4}$ as a colorless oil (56.6 mg, $89 \%$ yield).

${ }^{1} \mathrm{H}$ NMR (400 MHz, $\left.\mathrm{CDCl}_{3}\right) \delta 7.34-7.27(\mathrm{~m}, 2 \mathrm{H}), 7.24-7.18(\mathrm{~m}, 1 \mathrm{H}), 7.17-7.00$ $(\mathrm{m}, 6 \mathrm{H}), 2.68(\mathrm{dd}, J=10.6,4.6 \mathrm{~Hz}, 1 \mathrm{H}), 2.35(\mathrm{~s}, 3 \mathrm{H}), 2.04-1.82(\mathrm{~m}, 7 \mathrm{H}), 1.81-$ $1.70(\mathrm{~m}, 1 \mathrm{H}), 1.25(\mathrm{~s}, 6 \mathrm{H}), 1.25(\mathrm{~s}, 6 \mathrm{H}), 0.75-0.58(\mathrm{~m}, 2 \mathrm{H})$ (see spectrum) ${ }^{11} \mathrm{~B}$ NMR $\left(128 \mathrm{MHz}, \mathrm{CDCl}_{3}\right) \delta 33.3$ (see spectrum) ${ }^{13} \mathrm{C} \mathrm{NMR}\left(100 \mathrm{MHz}, \mathrm{CDCl}_{3}\right) \delta 142.7$, 138.9, 137.0, 130.5, 128.4, 128.2, 127.8, 126.7, 126.0, 125.7, 83.0, 51.1, 49.9, 43.8, $42.8,25.9,25.0,24.9,20.9$ (the signal for the carbon attached the boron was not observed) (see spectrum) $.[\alpha]_{\mathrm{D}}^{25}=+5\left(c=1, \mathrm{CHCl}_{3}\right)$. IR (film) $v_{\max } / \mathrm{cm}^{-1}: 2967,2923$, 2867, 1452, 1372, 1317, 1269, 1145, 1054, 1033, 968, 842, 750, 705. HRMS (ESI): Calcd. for $\mathrm{C}_{27} \mathrm{H}_{35} \mathrm{BNaO}_{2}\left([\mathrm{M}+\mathrm{Na}]^{+}\right) \mathrm{m} / \mathrm{z}$ 425.2627, found $\mathrm{m} / \mathrm{z} 425.2614$.

The ee of $\mathbf{3 4}$ was determined after hydroboration/oxidation to alcohol 34a. 


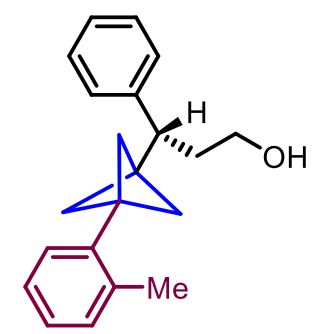

To a solution of hydroboration product $34(89.0 \mu \mathrm{mol})$ in $\mathrm{THF} / \mathrm{H}_{2} \mathrm{O}(1.8 \mathrm{~mL}, v / v 1 / 1)$ was added sodium perborate tetrahydrate $(0.448 \mathrm{mmol})$. The reaction mixture was stirred vigorously for $2 \mathrm{~h}$ at r.t. before quenching with water and extracting with EtOAc $(3 \times 5 \mathrm{~mL})$. The combined organic layers were dried over $\mathrm{MgSO}_{4}$ and concentrated under reduced pressure. The product was purified by flash column chromatography with hexanes/EtOAc (6/1 to 3/1) as eluent to give the alcohol as a colorless oil ( $25.7 \mathrm{mg}, 92 \%$ yield, $98 \%$ ee).

${ }^{1} \mathrm{H}$ NMR (400 MHz, $\left.\mathrm{CDCl}_{3}\right) \delta 7.39-7.28(\mathrm{~m}, 2 \mathrm{H}), 7.27-7.20(\mathrm{~m}, 1 \mathrm{H}), 7.20-7.14$ $(\mathrm{m}, 2 \mathrm{H}), 7.13-7.00(\mathrm{~m}, 4 \mathrm{H}), 3.60(\mathrm{ddd}, J=10.5,7.3,5.0 \mathrm{~Hz}, 1 \mathrm{H}), 3.51(\mathrm{ddd}, J=$ 10.5, 7.8, $6.7 \mathrm{~Hz}, 1 \mathrm{H}), 2.94(\mathrm{dd}, J=11.0,4.4 \mathrm{~Hz}, 1 \mathrm{H}), 2.34$ (s, 3H), $2.12-2.03$ (m, $1 \mathrm{H}), 2.01-1.89$ (m, 7H), 1.32 (br. s, 1H) (see spectrum $).{ }^{13} \mathrm{C}$ NMR $\left(100 \mathrm{MHz}, \mathrm{CDCl}_{3}\right)$ $\delta 141.9,138.6,137.0,130.5,128.5,128.1,127.8,126.8,126.5,125.8,61.5,50.8,43.7$, 43.6, 42. 8, 34.5, 20.9 (see spectrum) $) \cdot[\alpha]_{\mathrm{D}}^{25}=+18\left(c=1, \mathrm{CHCl}_{3}\right)$. IR (film) $v_{\max } / \mathrm{cm}^{-1}$ : 3336, 2961, 2927, 2866, 1602, 1489, 1453, 1378, 1262, 1159, 1028, 840, 746, 705. HRMS (ESI): Calcd. for $\mathrm{C}_{21} \mathrm{H}_{24} \mathrm{NaO}\left([\mathrm{M}+\mathrm{Na}]^{+}\right) \mathrm{m} / \mathrm{z} 315.1719$, found $\mathrm{m} / \mathrm{z} 315.1708$.

HPLC conditions: Chiral column IA, $n$-hexane $/ i-\mathrm{PrOH}=99: 1$, flow rate $=1.0$ $\mathrm{mL} / \mathrm{min}$, wavelength $=210 \mathrm{~nm}, t_{\mathrm{R}}=25.5 \mathrm{~min}$ for major isomer, $t_{\mathrm{R}}=27.4 \mathrm{~min}$ for minor isomer (see spectrum). 
(S)-2-(2-(4-Chlorophenyl)-2-(3-phenylbicyclo[1.1.1]pentan-1-yl)ethyl)isoindoline-

\section{1,3-dione (35)}
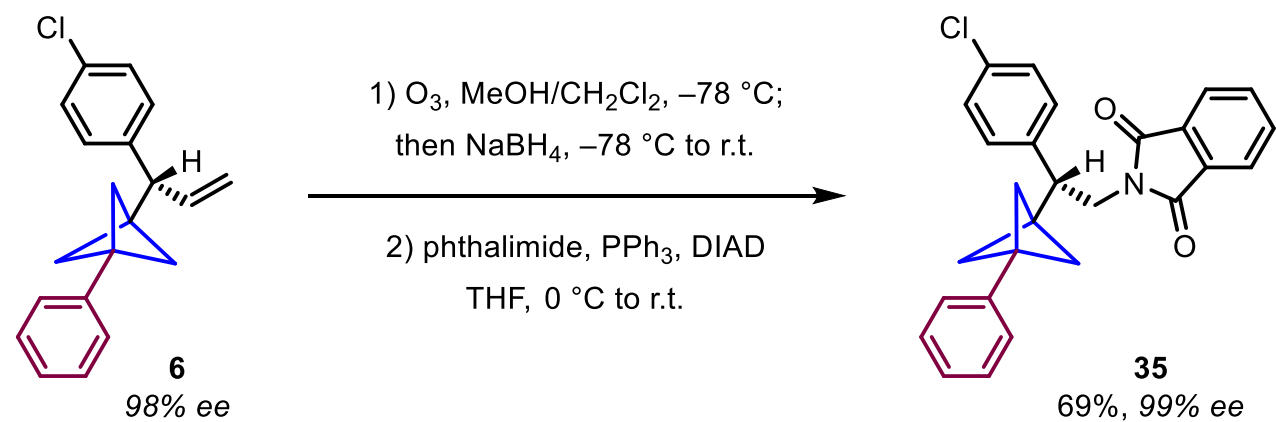

In a $20 \mathrm{~mL}$ vial, a solution of product $6(0.15 \mathrm{mmol})$ in $\mathrm{CH}_{2} \mathrm{Cl}_{2} / \mathrm{MeOH}(3 \mathrm{~mL}, 1: 1 \mathrm{v} / \mathrm{v})$ was cooled to $-78{ }^{\circ} \mathrm{C}$ and a stream of $\mathrm{O}_{3}-\mathrm{O}_{2}(\mathrm{~g})$ was bubbled through the reaction solution for $10 \mathrm{~min}$. The reaction mixture was then purged of $\mathrm{O}_{3}(\mathrm{~g})$ by bubbling $\mathrm{N}_{2}(\mathrm{~g})$ through the solution for $10 \mathrm{~min}$ followed by the addition of freshly ground $\mathrm{NaBH}_{4}$ $(0.30 \mathrm{mmol})$. The reaction was stirred at $-78^{\circ} \mathrm{C}$ for $30 \mathrm{~min}$, before removing from the cold bath and stirring for a further $30 \mathrm{~min}$. The reaction mixture was then poured over saturated brine solution $(3 \mathrm{~mL})$ and extracted with $\mathrm{CH}_{2} \mathrm{Cl}_{2}(3 \times 20 \mathrm{~mL})$. The organic phases were combined, dried over $\mathrm{Na}_{2} \mathrm{SO}_{4}$, filtered, and concentrated under reduced pressure. The crude product ( 1 equiv), phthalimide $(0.18 \mathrm{mmol}, 1.2$ equiv), and triphenylphosphine ( $0.18 \mathrm{mmol}, 1.2$ equiv) were added to a $5 \mathrm{~mL}$ vial under an inert atmosphere. The mixture was dissolved in THF $(3 \mathrm{~mL})$. The solution was cooled to $0{ }^{\circ} \mathrm{C}$ before diisopropyl azodicarboxylate (DIAD, $0.18 \mathrm{mmol}, 1.2$ equiv) was added dropwise. The reaction mixture was allowed to warm to r.t. and stirred for $2 \mathrm{~h}$. The THF was then removed under reduced pressure, and the mixture was diluted with EtOAc $(20 \mathrm{~mL})$ and water $(15 \mathrm{~mL})$. The phases were separated, and the aqueous layer was extracted with EtOAc $(2 \times 20 \mathrm{~mL})$. The organic phases were combined, dried over $\mathrm{Na}_{2} \mathrm{SO}_{4}$, filtered, and concentrated under reduced pressure. The residue was purified by column chromatography on silica gel with hexane/EtOAc (10/1) to afford the product as a solid (44.2 $\mathrm{mg}, 69 \%$ yield, $99 \%$ ee) over 2 steps.

${ }^{1} \mathrm{H}$ NMR (400 MHz, acetone- $\left.d_{6}\right) \delta 7.83-7.77(\mathrm{~m}, 4 \mathrm{H}), 7.34-7.26(\mathrm{~m}, 6 \mathrm{H}), 7.23-$ $7.16(\mathrm{~m}, 3 \mathrm{H}), 4.12(\mathrm{dd}, J=13.8,10.2 \mathrm{~Hz}, 1 \mathrm{H}), 3.99(\mathrm{dd}, J=13.8,6.3 \mathrm{~Hz}, 1 \mathrm{H}), 3.65$ $(\mathrm{dd}, J=10.1,6.3 \mathrm{~Hz}, 1 \mathrm{H}), 2.01-1.91(\mathrm{~m}, 6 \mathrm{H})$ (see spectrum) $){ }^{13} \mathrm{C}$ NMR $(100 \mathrm{MHz}$, acetone- $\left.d_{6}\right) \delta 168.6,141.6,139.3,135.1,132.9,132.8,130.9,129.1,129.0,127.2$, $126.7,123.7,51.5,45.1,42.9,41.1,40.5$ (see spectrum) $.[\alpha]_{\mathrm{D}}^{25}=+98(c=0.5$, $\left.\mathrm{MeOH} / \mathrm{CH}_{2} \mathrm{Cl}_{2}=1: 1\right)$. All spectroscopic data match those previously reported in the literature. ${ }^{3}$

HPLC conditions: Chiral column IA, $n$-hexane $/ i-\mathrm{PrOH}=95: 5$, flow rate $=1.0$ $\mathrm{mL} / \mathrm{min}$, wavelength $=210 \mathrm{~nm}, t_{\mathrm{R}}=17.1$ minor for major isomer, $t_{\mathrm{R}}=18.3 \mathrm{~min}$ for major isomer (see spectrum). 


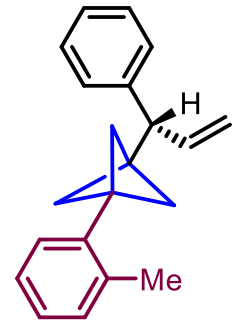

4

$98 \%$ ee

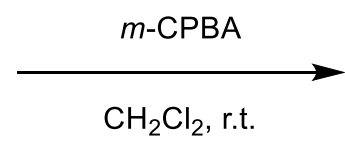

$83 \%, 54: 46 d r$

In a $5 \mathrm{~mL}$ vial, $m$-CPBA $(0.23 \mathrm{mmol})$ was added to a solution of alkene $4(0.15 \mathrm{mmol})$ in $\mathrm{CH}_{2} \mathrm{Cl}_{2}(2 \mathrm{~mL})$. The mixture was stirred at r.t. for $6 \mathrm{~h}$ before concentrating under reduced pressure. The residue was purified by column chromatography on silica gel with hexane/EtOAc (20/1) to afford the product in $83 \%$ yield $(36.1 \mathrm{mg})$. The dr was determined by ${ }^{1} \mathrm{H}$ NMR analysis to be $54: 46$.

${ }^{1} \mathrm{H}$ NMR (400 MHz, $\left.\mathrm{CDCl}_{3}\right) \delta(54: 46$ mixture of diastereomers) $7.29-7.23(\mathrm{~m}, 2 \mathrm{H})$, $7.22-7.14(\mathrm{~m}, 2 \mathrm{H}), 7.13-7.07(\mathrm{~m}, 1 \mathrm{H}), 7.06-6.96(\mathrm{~m}, 4 \mathrm{H}), 3.29-3.18(\mathrm{~m}, 1 \mathrm{H})$, $2.81(\mathrm{dd}, J=5.0,3.9 \mathrm{~Hz}, 0.46 \mathrm{H}), 2.67(\mathrm{dd}, J=4.9,3.9 \mathrm{~Hz}, 0.54 \mathrm{H}), 2.54(\mathrm{~d}, J=7.4$ $\mathrm{Hz}, 0.46 \mathrm{H}), 2.46$ (dd, $J=5.0,2.6 \mathrm{~Hz}, 0.46 \mathrm{H}), 2.39$ (dd, $J=4.9,2.7 \mathrm{~Hz}, 0.54 \mathrm{H}), 2.34$ (d, $J=8.6 \mathrm{~Hz}, 0.54 \mathrm{H}), 2.28+2.27(2 \times \mathrm{s}, 3 \mathrm{H}), 2.09-1.93(\mathrm{~m}, 6 \mathrm{H})$ (see spectrum). ${ }^{13} \mathrm{C}$ NMR $\left(100 \mathrm{MHz}, \mathrm{CDCl}_{3}\right)$ Major isomer: $\delta$ 139.5, 138.3, 136.9, 130.5, 128.5, 128.0, 127.7, 126.81, 126.76, 125.7, 53.7, 51.6, 50.6, 46.0, 43.7, 42.1, 20.7; Minor isomer: $\delta 139.1,138.1,136.8,130.5,128.4,128.3,127.7,126.9,126.8,125.7,53.2$, 51.7, 49.8, 46.8, 43.7, 41.3, 20.7 (see spectrum). HRMS (APCI): Calcd. for $\mathrm{C}_{21} \mathrm{H}_{23} \mathrm{O}$ $\left([\mathrm{M}+\mathrm{H}]^{+}\right) \mathrm{m} / \mathrm{z} 291.1743$, found $\mathrm{m} / \mathrm{z} 291.1737$.

(3S)-3-Phenyl-3-(3-(o-tolyl)bicyclo[1.1.1]pentan-1-yl)propane-1,2-diol (37)

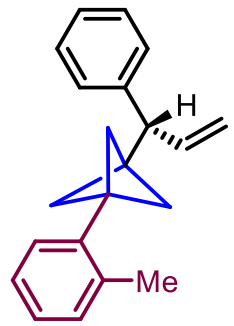

4

$98 \%$ ee

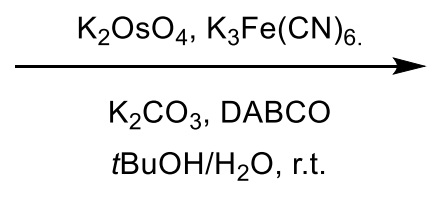

tBuOH$/ \mathrm{H}_{2} \mathrm{O}$, r.t.

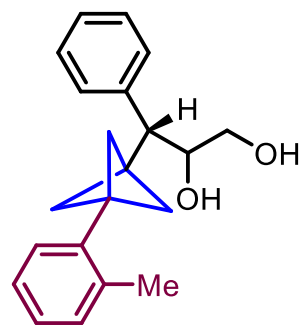

37

$83 \%, 78: 22 d r$

To a solution of potassium osmate(VI) dihydrate (5 mol\%), potassium hexacyanoferrate(III) $(0.30 \mathrm{mmol})$, and $\mathrm{K}_{2} \mathrm{CO}_{3}(0.30 \mathrm{mmol})$ in water $(1.0 \mathrm{~mL})$ at $0{ }^{\circ} \mathrm{C}$ was added a solution of alkene $4(0.10 \mathrm{mmol})$ in tert-butanol $(1.0 \mathrm{~mL})$. Then DABCO $(0.10 \mathrm{mmol})$ was added and the reaction stirred at r.t. for $7 \mathrm{~h}$. The mixture was extracted with $\mathrm{CH}_{2} \mathrm{Cl}_{2}(3 \times 5 \mathrm{~mL})$. The combined organic phases were washed with 
water and brine, dried over $\mathrm{MgSO}_{4}$, filtered, and concentrated under reduced pressure. The product was purified by flash column chromatography with hexanes/EtOAc (4/1 to $1 / 1)$ as eluent to give 37 as a colorless oil in $83 \%$ yield $(41.1 \mathrm{mg})$. The $\mathrm{dr}$ was determined by ${ }^{1} \mathrm{H}$ NMR of the crude reaction mixture to be 78:22.

${ }^{1} \mathrm{H}$ NMR (400 MHz, $\mathrm{CDCl}_{3}$ ) (78:22 mixture of diastereomers) $\delta 7.34-7.21(\mathrm{~m}, 2 \mathrm{H})$, $7.23-7.11(\mathrm{~m}, 3 \mathrm{H}), 7.04-7.00(\mathrm{~m}, 2 \mathrm{H}), 7.00-6.91(\mathrm{~m}, 2 \mathrm{H}), 4.13-3.95(\mathrm{~m}, 1 \mathrm{H})$, $3.77(\mathrm{ddd}, J=9.8,5.8,3.0 \mathrm{~Hz}, 0.78 \mathrm{H}), 3.48(\mathrm{ddd}, J=10.9,7.0,3.6 \mathrm{~Hz}, 0.78 \mathrm{H}), 3.41$ $-3.31(\mathrm{~m}, 0.22 \mathrm{H}), 3.23-3.15(\mathrm{~m}, 0.22 \mathrm{H}), 2.84(\mathrm{~d}, J=7.6 \mathrm{~Hz}, 0.78 \mathrm{H}), 2.77(\mathrm{~d}, J=$ $9.6 \mathrm{~Hz}, 0.22 \mathrm{H}$ ), 2.36 (br. d, $J=4.9 \mathrm{~Hz}, 1 \mathrm{H}), 2.27$ (s, $0.66 \mathrm{H}), 2.25$ (s, 2.34H), $2.15-$ 2.07 (br. m, 0.78H), 2.04 (d, $J=1.6 \mathrm{~Hz}, 0.22 \mathrm{H}), 2.03-1.91(\mathrm{~m}, 6 \mathrm{H}), 1.85$ (d, $J=3.5$ $\mathrm{Hz}, 0.78 \mathrm{H})$ (see spectrum $).{ }^{13} \mathrm{C}$ NMR $\left(100 \mathrm{MHz}, \mathrm{CDCl}_{3}\right)$ Major isomer: $\delta 139.3,138.2$, 136.9, 130.6, 129.2, 128.8, 127.8, 127.1, 127.0, 125.8, 73.1, 65.8, 52.4, 50.0, 43.6, 41.3, 20.8; Minor isomer: $\delta 139.9,138.4,136.9,130.5,128.7,128.5,127.8,126.9$, 126.8, 125.8, 74.5, 65.6, 52.5, 50.3, 43.7, 42.0, 20.9 (see spectrum). HRMS (ESI): Calcd. for $\mathrm{C}_{21} \mathrm{H}_{24} \mathrm{NaO}_{2}\left([\mathrm{M}+\mathrm{Na}]^{+}\right) \mathrm{m} / \mathrm{z} 331.1669$, found $\mathrm{m} / \mathrm{z} 331.1671$.

\section{Dimethyl 2-((S)-phenyl(3-(o-tolyl)bicyclo[1.1.1]pentan-1-yl)methyl)cyclopropane-}

\section{1,1-dicarboxylate (38)}

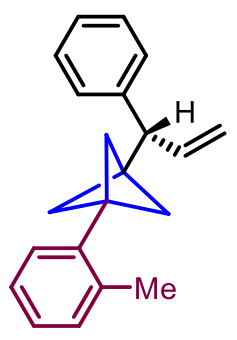

4

$98 \%$ ee

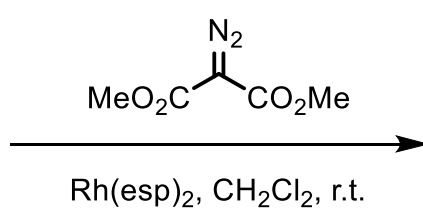

$\mathrm{Rh}(\mathrm{esp})_{2}, \mathrm{CH}_{2} \mathrm{Cl}_{2}$, r.t.

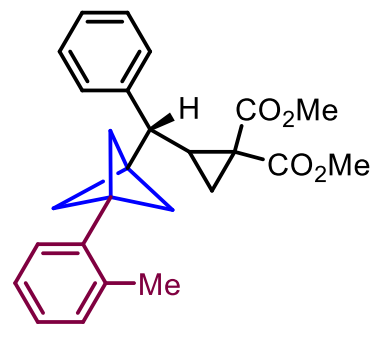

38

$88 \%, 62: 38 d r$

In a $5 \mathrm{~mL}$ vial, $\mathrm{Rh}_{2}(\mathrm{esp})_{2}(1.0 \mathrm{~mol} \%)$ and $\mathrm{CH}_{2} \mathrm{Cl}_{2}(1 \mathrm{~mL})$ were added and stirred for 20 min under an inert atmosphere. Alkene $4(0.15 \mathrm{mmol})$ was added, and the mixture was then cooled to $0{ }^{\circ} \mathrm{C}$. A solution of dimethyl diazomalonate $(0.30 \mathrm{mmol})$ in $\mathrm{CH}_{2} \mathrm{Cl}_{2}(1 \mathrm{~mL})$ was added. The resulting stirred at $0{ }^{\circ} \mathrm{C}$ for $10 \mathrm{~min}$ and then at r.t. for $2 \mathrm{~h}$. The mixture was concentrated under reduced pressure and the residue was purified by column chromatography on silica gel with hexane/EtOAc (20/1) to afford the product in $88 \%$ yield $(53.5 \mathrm{mg})$. The $\mathrm{dr}$ was determined by ${ }^{1} \mathrm{H}$ NMR analysis to be $62: 38$.

${ }^{1} \mathrm{H}$ NMR (400 MHz, $\mathrm{CDCl}_{3}$ ) (62:38 mixture of diastereomers) $\delta 7.28-7.09(\mathrm{~m}, 4 \mathrm{H})$, $7.07-6.94(\mathrm{~m}, 5 \mathrm{H}), 3.75(\mathrm{~s}, 1.14 \mathrm{H}), 3.71(\mathrm{~s}, 1.14 \mathrm{H}), 3.64(\mathrm{~s}, 1.86 \mathrm{H}), 3.25(\mathrm{~s}, 1.86$ H), $2.53(\mathrm{~d}, J=10.8 \mathrm{~Hz}, 0.38 \mathrm{H}), 2.45-2.28(\mathrm{~m}, 1.62 \mathrm{H}), 2.25(\mathrm{~s}, 1.86 \mathrm{H}), 2.25(\mathrm{~s}$, $1.14 \mathrm{H}), 1.95-1.81(\mathrm{~m}, 6 \mathrm{H}), 1.66-1.61(\mathrm{~m}, 0.62), 1.52-1.47(0.62 \mathrm{H}), 1.33-1.28$ $(0.76 \mathrm{H})$ (see spectrum $).{ }^{13} \mathrm{C} \mathrm{NMR}\left(100 \mathrm{MHz}, \mathrm{CDCl}_{3}\right)$ Major isomer: $\delta$ 170.6, 168.6, 
$140.6,138.2,136.8,130.4,128.1,127.7,127.5,126.8,126.4,125.7,52.7,52.0,50.9$, 46.4, 43.2, 42.8, 32.7, 29.6, 22.1, 20.7; Minor isomer: $\delta$ 170.3, 168.9, 141.7, 138.3, $136.8,130.4,128.3,128.0,127.7,126.7,126.5,125.6,52.8,52.5,51.0,44.5,43.2$, 42.9, 35.2, 30.9, 22.1, 20.0 (see spectrum). HRMS (APCI): Calcd. for $\mathrm{C}_{26} \mathrm{H}_{29} \mathrm{O}_{4}$ $\left([\mathrm{M}+\mathrm{H}]^{+}\right) \mathrm{m} / \mathrm{z}$ 406.2060, found $\mathrm{m} / \mathrm{z} 406.2047$.

\section{Methyl (S)-2,2-difluoro-5-phenyl-5-(3-(o-tolyl)bicyclo[1.1.1]pentan-}

\section{1-yl)pentanoate (39)}

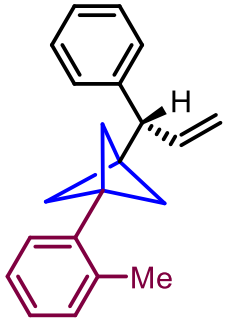

4

$98 \%$ ee

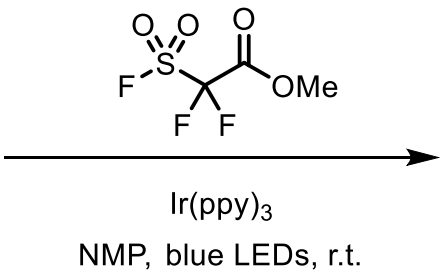

NMP, blue LEDs, r.t.

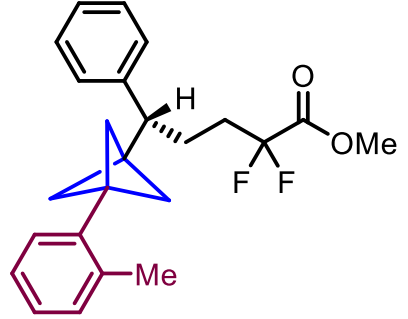

39

$80 \%, 98 \%$ ee

To a $10 \mathrm{~mL}$ Schlenk tube was added $f a c-\operatorname{Ir}(\mathrm{ppy})_{3} \quad(3.0 \mathrm{~mol} \%)$, methyl fluorosulfonyldifluoroacetate $(0.20 \mathrm{mmol})$, and dry $N$-methyl-2-pyrrolidone (NMP) $(8.0 \mathrm{~mL})$, followed by alkene $4(0.40 \mathrm{mmol})$. The mixture was degassed by three freeze-pump-thaw cycles before it was stirred at r.t. for $1 \mathrm{~h}$. The flask was placed at a distance of $2 \mathrm{~cm}$ from the blue LEDs and irradiated for $24 \mathrm{~h}$. The reaction was quenched with water $(20 \mathrm{~mL})$ and extracted with $\mathrm{Et}_{2} \mathrm{O}(3 \times 20 \mathrm{~mL})$. The combined organic phases were dried over $\mathrm{MgSO}_{4}$, filtered, and concentrated under reduced pressure. The residue was purified by column chromatography with pentane/ $/ \mathrm{Et}_{2} \mathrm{O}$ (100/0 to 20/1) as eluent to give 39 as a colorless oil (48.7 mg, 80\% yield, 98\% ee).

${ }^{1} \mathrm{H}$ NMR (400 MHz, $\left.\mathrm{CDCl}_{3}\right) \delta 7.38-7.30(\mathrm{~m}, 2 \mathrm{H}), 7.28-7.22(\mathrm{~m}, 1 \mathrm{H}), 7.16-7.07$ $(\mathrm{m}, 4 \mathrm{H}), 7.08-7.01(\mathrm{~m}, 2 \mathrm{H}), 3.85(\mathrm{~s}, 3 \mathrm{H}), 2.76(\mathrm{dd}, J=11.5,3.2 \mathrm{~Hz}, 1 \mathrm{H}), 2.33(\mathrm{~s}$, $3 \mathrm{H}), 2.13-1.70(\mathrm{~m}, 10 \mathrm{H})$ (see spectrum) ${ }^{19} \mathrm{~F}$ NMR $\left(377 \mathrm{MHz}, \mathrm{CDCl}_{3}\right) \delta-105.67-$ $-106.37(\mathrm{~m})$ (see spectrum) ${ }^{13} \mathrm{C} \mathrm{NMR}\left(100 \mathrm{MHz}, \mathrm{CDCl}_{3}\right) \delta 164.9(\mathrm{t}, J=33.2 \mathrm{~Hz})$, $141.1,138.4,137.0,130.6,128.6,128.0,127.8,126.9,126.7,125.8,116.5(\mathrm{t}, J=$ $250.0 \mathrm{~Hz}), 53.4,50.9,46.7,43.6,42.8,33.1(\mathrm{t}, J=23.1 \mathrm{~Hz}), 23.2(\mathrm{t}, J=3.9 \mathrm{~Hz}), 20.8$ (see spectrum) $.[\alpha]_{\mathrm{D}}^{25}=+13\left(c=0.5, \mathrm{CHCl}_{3}\right)$. IR (film) $v_{\max } / \mathrm{cm}^{-1}: 3026,2962,2906$, 2868, 1771, 1492, 1454, 1307, 1264, 1203, 1087, 1040, 818, 748, 704. HRMS (ESI): Calcd. for $\mathrm{C}_{24} \mathrm{H}_{26} \mathrm{~F}_{2} \mathrm{NaO}_{2}\left([\mathrm{M}+\mathrm{Na}]^{+}\right) \mathrm{m} / \mathrm{z}$ 407.1793, found $\mathrm{m} / \mathrm{z}$ 407.1797.

HPLC conditions: Chiral column IA, $n$-hexane $/ i-\mathrm{PrOH}=99: 1$, flow rate $=0.7$ $\mathrm{mL} / \mathrm{min}$, wavelength $=210 \mathrm{~nm}, t_{\mathrm{R}}=7.3 \mathrm{~min}$ for major isomer, $t_{\mathrm{R}}=6.5 \mathrm{~min}$ for minor isomer (see spectrum). 
(40)

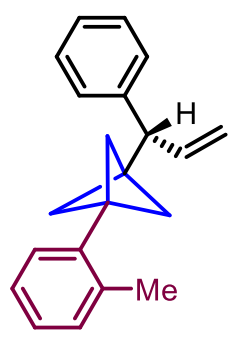

4

$98 \%$ ee

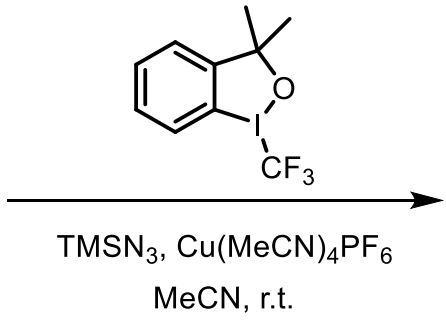

$\mathrm{MeCN}$, r.t.

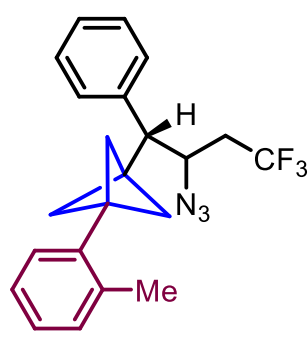

40

$84 \%, 60: 40 d r$

In a $5 \mathrm{~mL}$ vial, Togni reagent $\mathrm{II}(0.30 \mathrm{mmol})$ and $\mathrm{Cu}(\mathrm{MeCN})_{4} \mathrm{PF}_{6}(10 \mathrm{~mol} \%)$ were dissolved in $\mathrm{MeCN}(2.0 \mathrm{~mL})$ under inert atmosphere. Alkene $4(0.15 \mathrm{mmol})$ and $\mathrm{TMSN}_{3}(0.30 \mathrm{mmol})$ were added. The mixture was stirred at r.t. for $12 \mathrm{~h}$ before concentrating under vacuum. The residue was purified by column chromatography on silica gel with hexane/EtOAc (200/1) to afford the product $\mathbf{4 0}$ in $84 \%$ yield (45.0 mg). The dr was determined by ${ }^{1} \mathrm{H}$ NMR analysis to be 60:40.

${ }^{1} \mathrm{H}$ NMR (400 MHz, $\mathrm{CDCl}_{3}$ ) (60:40 mixture of diastereomers) $\delta 7.45-7.27(\mathrm{~m}, 4 \mathrm{H})$, $7.16-7.05$ (m, 5H), 4.13 (ddd, $J=8.6,5.0,3.2 \mathrm{~Hz}, 0.40 \mathrm{H}), 3.89$ (ddd, $J=10.2,7.2$, $5.2 \mathrm{~Hz}, 0.60 \mathrm{H}), 2.90(\mathrm{~d}, J=4.9 \mathrm{~Hz}, 0.40 \mathrm{H}), 2.86(\mathrm{~d}, J=10.2 \mathrm{~Hz}, 0.60 \mathrm{H}), 2.37$ (s, $1.20 \mathrm{H}), 2.36(\mathrm{~s}, 1.80 \mathrm{H}), 2.33-2.03(\mathrm{~m}, 8 \mathrm{H})$ (see spectrum) ${ }^{19} \mathrm{~F}$ NMR $(377 \mathrm{MHz}$, $\mathrm{CDCl}_{3}$ ) Major isomer: $\delta-64.2(\mathrm{~s})$; Minor isomer: $\delta-64.1$ (s) (see spectrum). ${ }^{13} \mathrm{C}$ NMR $\left(100 \mathrm{MHz}, \mathrm{CDCl}_{3}\right)$ Major isomer: $\delta 138.7,137.8,136.8,130.5,129.1,128.1,127.7$, 127.5, 126.9, $126.0(\mathrm{q}, J=277.2 \mathrm{~Hz}), 125.7,59.5(\mathrm{q}, J=2.3 \mathrm{~Hz}), 52.2,51.1,43.5$, 41.6, 37.5 (q, $J=28.0 \mathrm{~Hz}$ ), 20.7; Minor isomer: $\delta$ 137.7, 137.1, 136.8, 130.5, 129.5, $128.5,127.7,127.4,127.0,126.0(\mathrm{q}, J=277.1 \mathrm{~Hz}), 125.8,59.2(\mathrm{q}, J=2.4 \mathrm{~Hz}), 52.2$, 51.5, 43.5, 41.8, 38.4 (q, $J=28.0 \mathrm{~Hz}$ ), 20.7 (see spectrum). HRMS (APCI): Calcd. for $\mathrm{C}_{22} \mathrm{H}_{23} \mathrm{NF}_{3}\left(\left[\mathrm{M}+\mathrm{H}-\mathrm{N}_{2}\right]^{+}\right) \mathrm{m} / \mathrm{z}$ 358.1777, found $\mathrm{m} / \mathrm{z} 358.1777$.

\section{Scale-up Reaction}

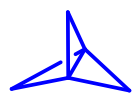

1

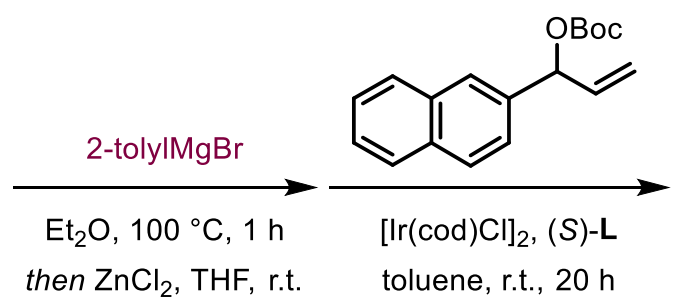

toluene, r.t., $20 \mathrm{~h}$
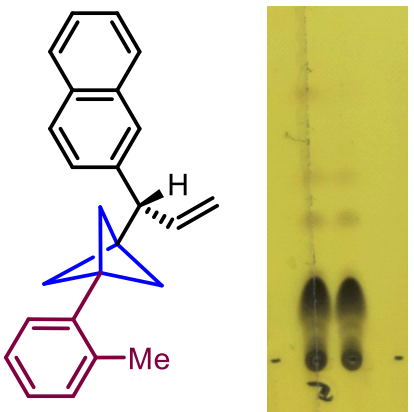

To a $50 \mathrm{~mL}$ pressure tube was added aryl magnesium bromide (2.0 $\mathrm{M}$ in $\mathrm{Et}_{2} \mathrm{O}, 1.6$ mmol, 1.6 equiv) and [1.1.1]propellane $\left(0.5 \mathrm{M}\right.$ in $\left.\mathrm{Et}_{2} \mathrm{O}, 3.2 \mathrm{mmol}\right)$ at r.t. under an 
inert atmosphere. The pressure tube was sealed and the solution was heated to $100{ }^{\circ} \mathrm{C}$ and stirred for $1 \mathrm{~h}$. The solvent was removed under high vacuum before $\mathrm{ZnCl}_{2}(0.5 \mathrm{M}$ in THF, $2.0 \mathrm{mmol}$, 2.0 equiv) was added and the mixture stirred for $20 \mathrm{~min}$ at r.t. The solvent was removed under high vacuum and toluene $(2.0 \mathrm{~mL})$ was added. To a separate $5 \mathrm{~mL}$ vial was added $[\operatorname{Ir}(\operatorname{cod}) \mathrm{Cl}]_{2}(13.5 \mathrm{mg}, 0.020 \mathrm{mmol}, 2.0 \mathrm{~mol} \%)$, phosphoramidite ligand $(S)-\mathbf{L}(40.5 \mathrm{mg}, 0.080 \mathrm{mmol}, 8.0 \mathrm{~mol} \%)$ and toluene $(3.0 \mathrm{~mL})$ under an inert atmosphere, and the mixture was vigorously stirred for 20 min before adding the allyl carbonates $(1.0 \mathrm{mmol}, 1.0$ equiv). The resulting mixture of the catalyst and substrate was then added to the pressure tube and the resulting mixture was stirred at r.t. for $20 \mathrm{~h}$. Saturated aqueous $\mathrm{NH}_{4} \mathrm{Cl}(15 \mathrm{~mL})$ was added, the mixture was extracted with $\mathrm{Et}_{2} \mathrm{O}(3 \times 20 \mathrm{~mL})$, and the combined organic phases were dried over $\mathrm{Na}_{2} \mathrm{SO}_{4}$, filtered, and concentrated under reduced pressure. Purification by flash column chromatography using pentane as the eluent gave the corresponding product ( $242 \mathrm{mg}, 75 \%,>99 \%$ ee). The enantioselectivity was determined by chiral HPLC.

HPLC conditions: Chiral column IB, $n$-hexane, flow rate $=1.0 \mathrm{~mL} / \mathrm{min}$, wavelength $=$ $210 \mathrm{~nm}, t_{\mathrm{R}}=14.5 \mathrm{~min}$ for minor isomer, $t_{\mathrm{R}}=19.5 \mathrm{~min}$ for major isomer

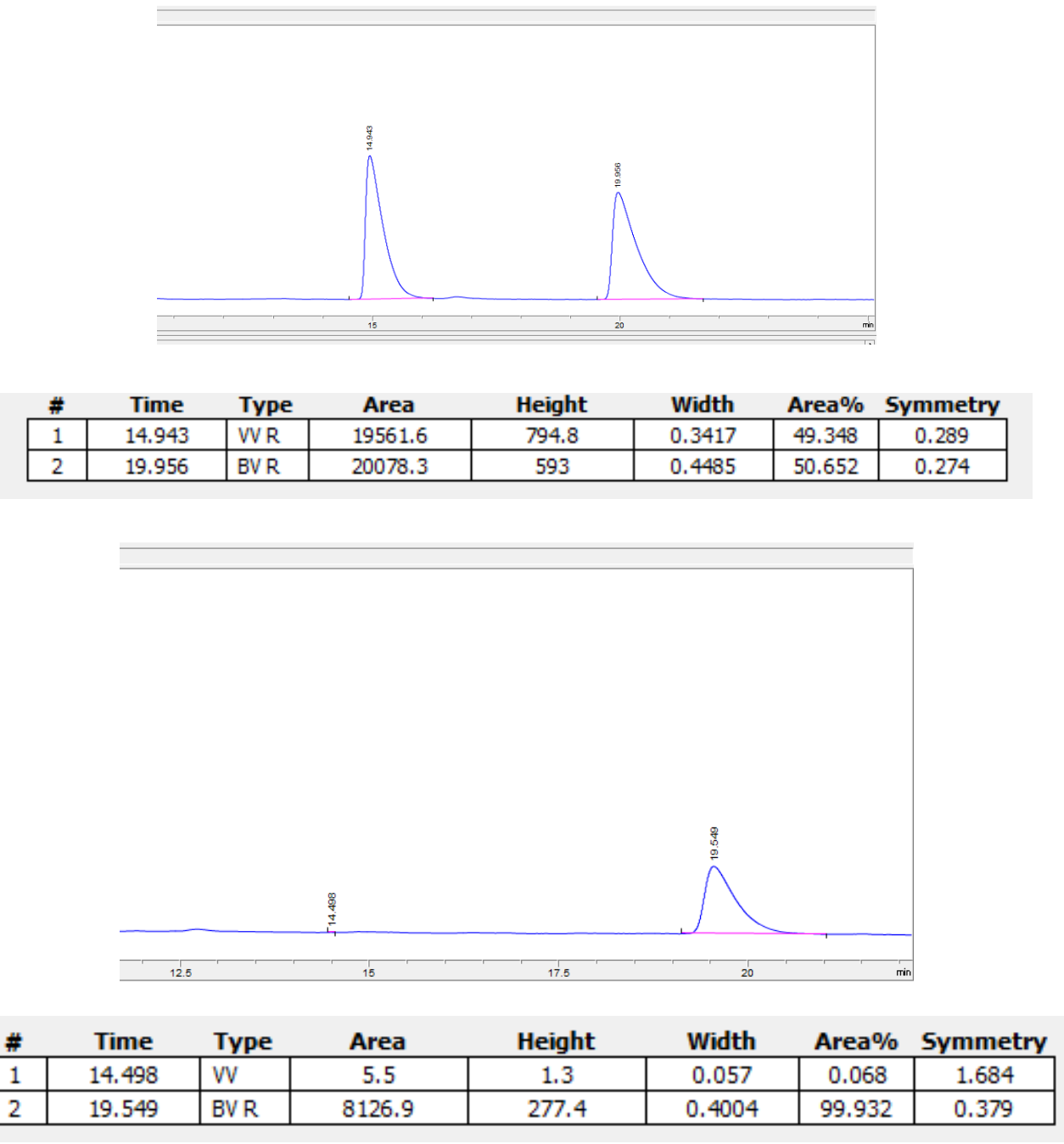


IV. References

1) Hamilton, J. Y.; Sarlah, D.; Carreira, E. M. Iridium-Catalyzed Enantioselective Allylic Alkylation with Functionalized Organozinc Bromides. Angew. Chem., Int. Ed. 2015, 54, 7644.

2) Yu, S.; Jing, C.; Noble, A.; Aggarwal, V. K. 1,3-Difunctionalizations of [1.1.1]Propellane via 1,2-Metallate Rear-rangements of Boronate Complexes. Angew. Chem. Int. Ed. 2020, 59, 3917.

3) Garlets, Z. J.; Sanders, J.N.; Malik, H.; Gampe, C.; Houk, K. N.; Davie, H. M. L. Enantioselective C-H functionalization of bicy-clo[1.1.1]pentanes. Nat. Catal. 2020, 3, 351 . 


\section{HPLC Spectra}

HPLC data for product 4 (see procedure):
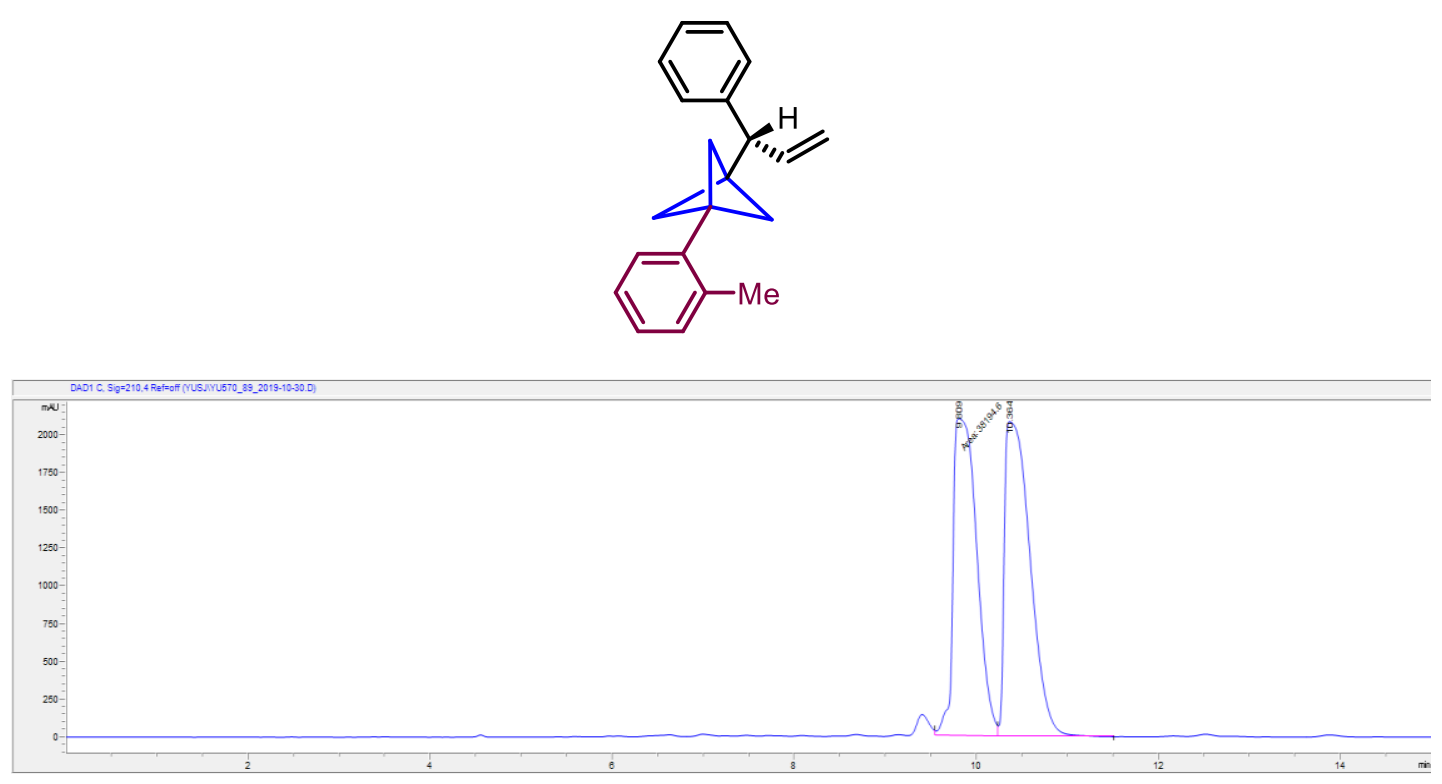

\begin{tabular}{|c|c|l|c|c|c|c|c|} 
\# & Time & \multicolumn{1}{c}{ Type } & \multicolumn{1}{c}{ Area } & \multicolumn{1}{c}{ Height } & \multicolumn{1}{c}{ Width } & \multicolumn{2}{c}{ Area\% $\%$ Symmetry } \\
\hline 1 & 9.809 & FM & 38194.6 & 2106.2 & 0.3022 & 48.398 & 0.353 \\
\hline 2 & 10.364 & VB & 40722.9 & 2088.5 & 0.3095 & 51.602 & 0.25 \\
\hline
\end{tabular}

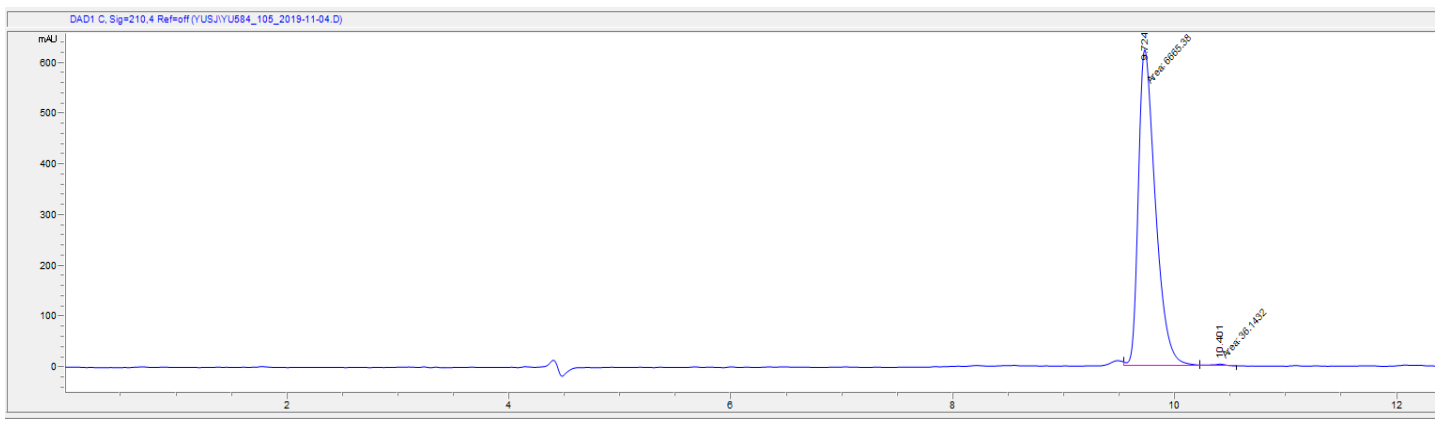

\begin{tabular}{|c|c|l|c|c|c|c|c|} 
\# & Time & \multicolumn{1}{c}{ Type } & Area & Height & \multicolumn{1}{c}{ Width } & \multicolumn{2}{c}{ Area\% } \\
\hline 1 & 9.724 & FM & 6665.4 & 623.5 & 0.1782 & 99.461 & 0.577 \\
\hline 2 & 10.401 & FM & 36.1 & 3.7 & 0.1632 & 0.539 & 1.712 \\
\hline
\end{tabular}


HPLC data for product 33 (see procedure):
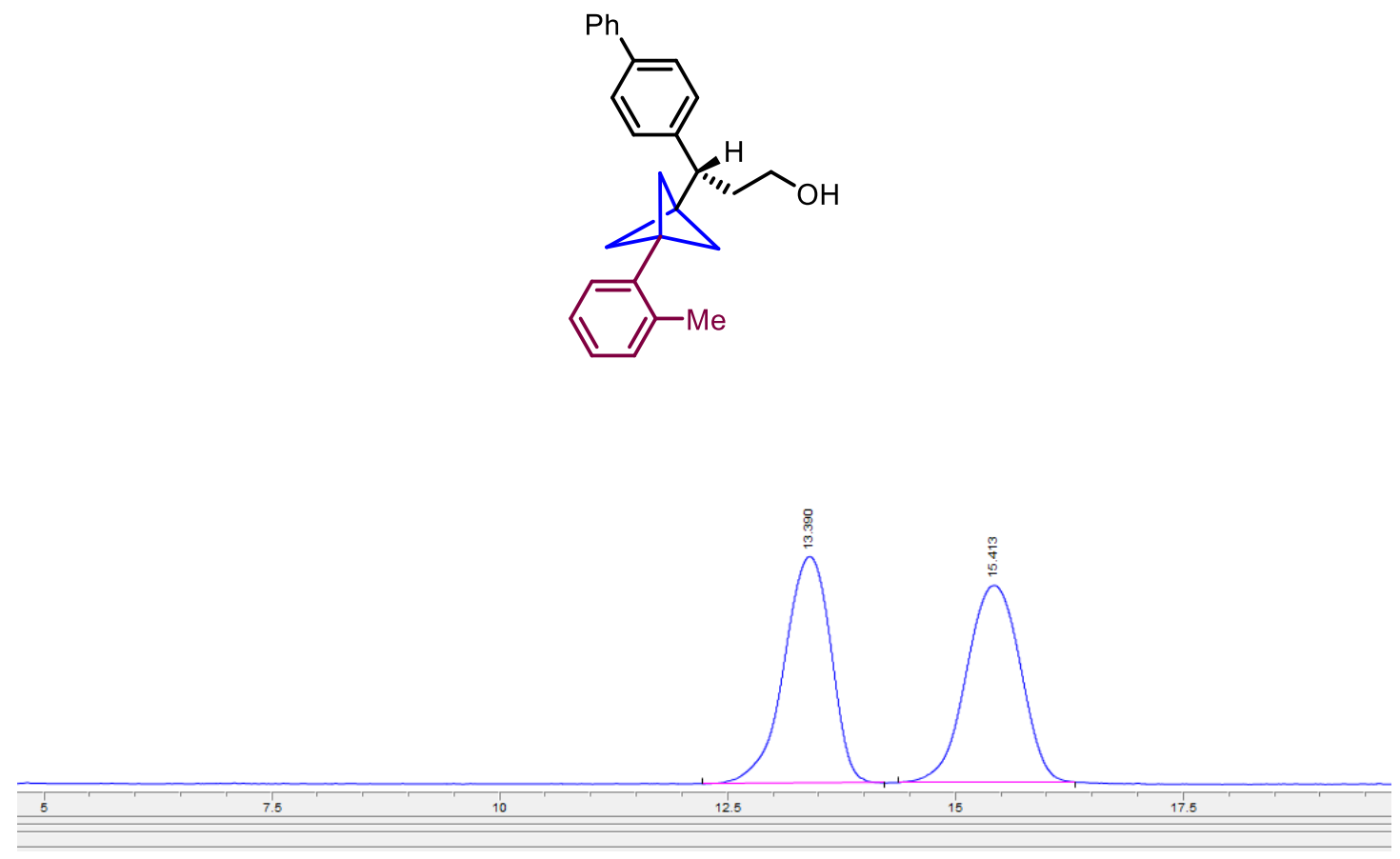

\begin{tabular}{|c|c|c|c|c|c|c|c|}
\hline$\#$ & Time & Type & Area & Height & Width & Area $\%$ & Symmetry \\
\hline 1 & 13.39 & VVR & 17771 & 495.2 & 0.4197 & 49.980 & 1.154 \\
\hline 2 & 15.413 & VVR & 17784.9 & 430.8 & 0.4847 & 50.020 & 1.016 \\
\hline
\end{tabular}

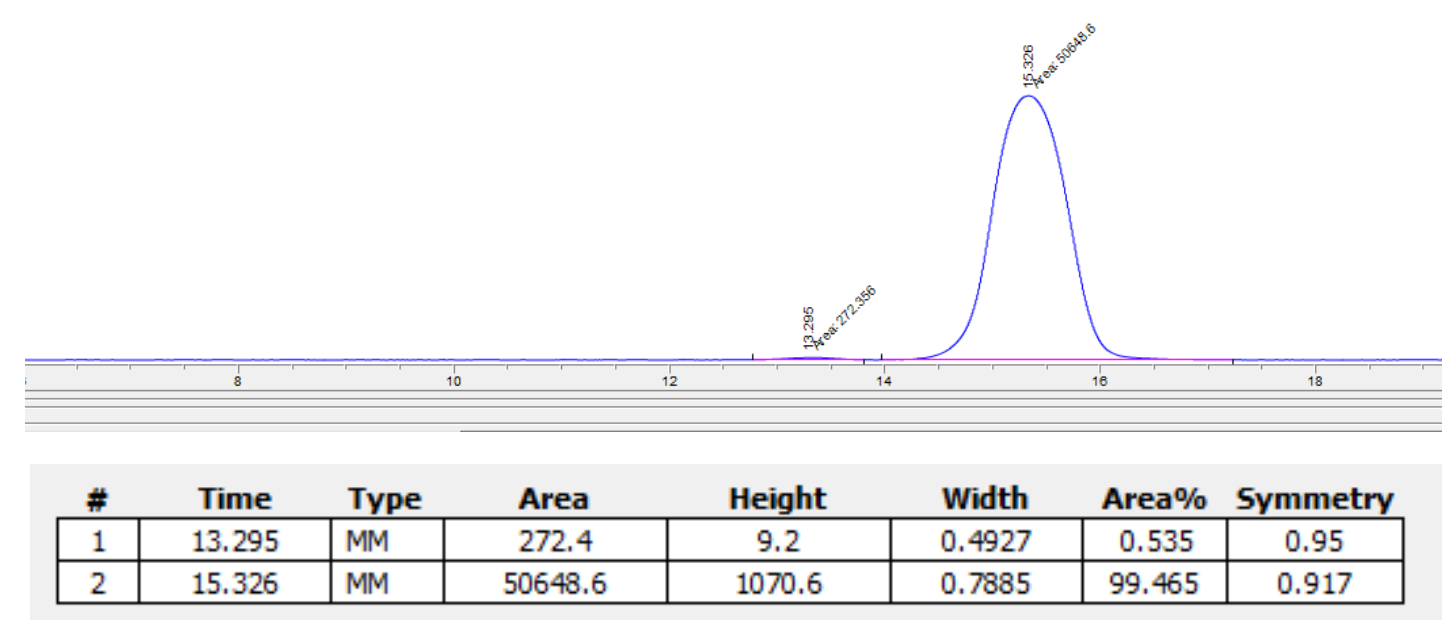


HPLC data for product 6 (see procedure):
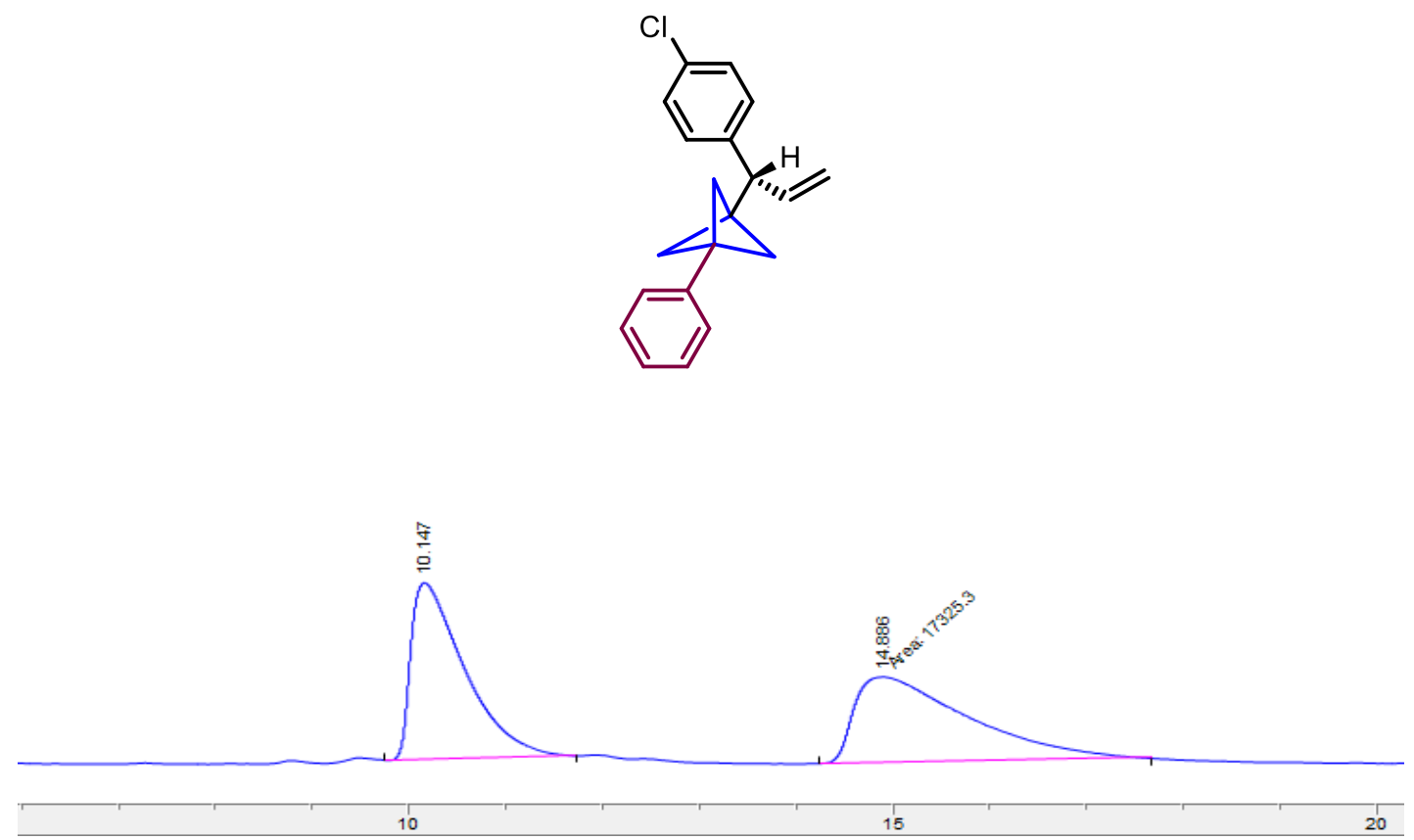

\begin{tabular}{|c|c|l|c|c|c|c|c|} 
\# & Time & \multicolumn{1}{c}{ Type } & \multicolumn{1}{c}{ Area } & \multicolumn{1}{c}{ Height } & \multicolumn{1}{c}{ Width } & \multicolumn{2}{c}{ Area\% } \\
\hline 1 & 10.147 & BB & 16932.3 & 438.7 & 0.4583 & 49.426 & 0.315 \\
\hline 2 & 14.886 & MM & 17325.3 & 212.4 & 1.3598 & 50.574 & 0.319 \\
\hline
\end{tabular}

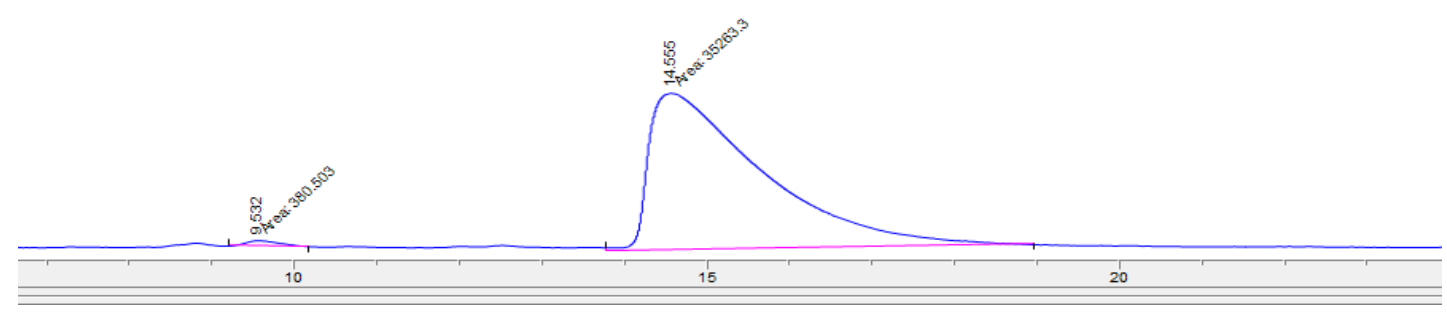

\begin{tabular}{|c|c|c|c|c|c|c|c|}
\hline$\#$ & Time & Type & Area & Height & Width & Area $\%$ & Symmetry \\
\hline 1 & 9.532 & $\mathrm{MM}$ & 380.5 & 13 & 0.4865 & 1.068 & 0.516 \\
\hline 2 & 14.555 & MM & 35263.3 & 367.6 & 1.5989 & 98.932 & 0.235 \\
\hline
\end{tabular}


HPLC data for product 7 (see procedure):
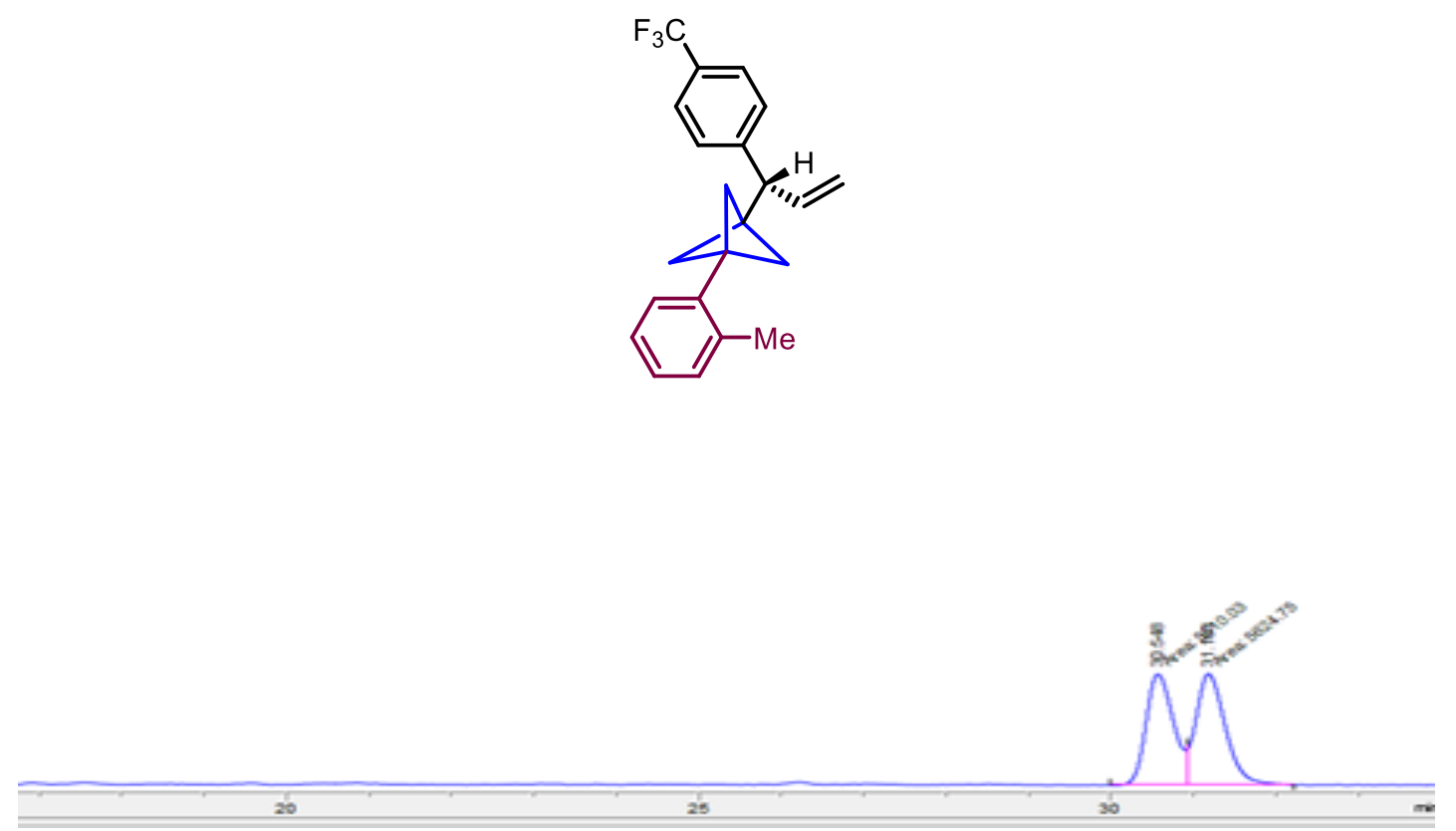

\begin{tabular}{|c|c|l|c|c|c|c|c|} 
\# & Time & \multicolumn{1}{c}{ Type } & \multicolumn{1}{c}{ Area } & Height & \multicolumn{1}{c}{ Width } & \multicolumn{2}{c|}{ Area\% } \\
\hline 1 & 30.548 & FM & 5510 & 229.3 & 0.4005 & 49.485 & 0.703 \\
\hline 2 & 31.163 & FM & 5624.8 & 230.3 & 0.407 & 50.515 & 0.674 \\
\hline
\end{tabular}

\begin{tabular}{|c|c|c|c|c|c|c|c|}
\hline & & & & & & 30 & \\
\hline$\#$ & Time & Type & Area & Height & Width & Area \% & Symmetry \\
\hline 1 & 30.487 & BVE & 6.6 & 1.3 & 0.0661 & 0.064 & 1.259 \\
\hline 2 & 31.122 & VVR & 10309.2 & 398.9 & 0.382 & 99.936 & 0.631 \\
\hline
\end{tabular}


HPLC data for product $8 \mathbf{a}$ (see procedure):
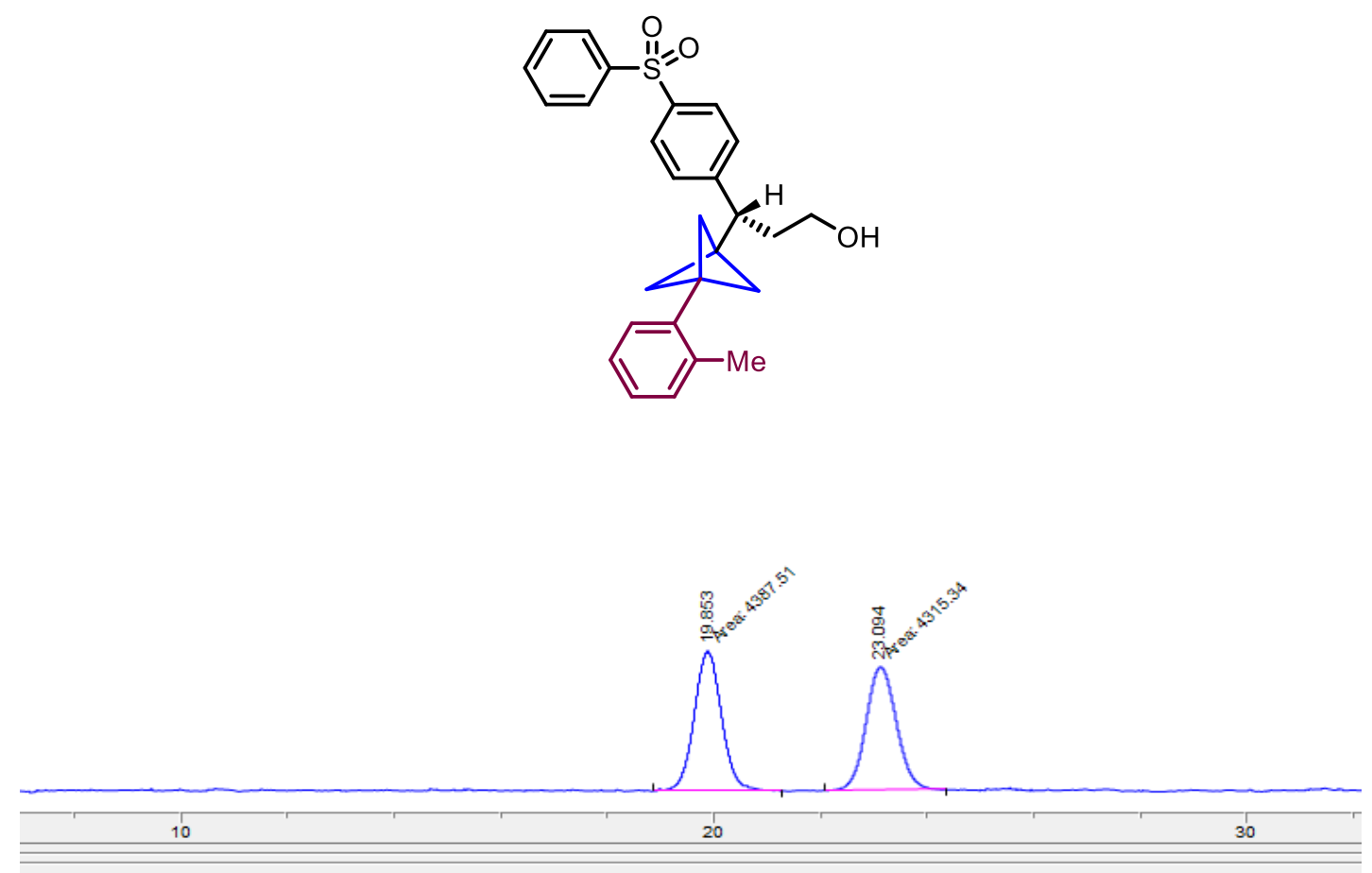

\begin{tabular}{|c|c|c|c|c|c|c|c|}
\hline$\#$ & Time & Type & Area & Height & Width & Area $\%$ & Symmetry \\
\hline 1 & 19.853 & MM & 4387.5 & 122.9 & 0.5951 & 50.415 & 0.955 \\
\hline 2 & 23.094 & MM & 4315.3 & 108.4 & 0.6635 & 49.585 & 0.898 \\
\hline
\end{tabular}

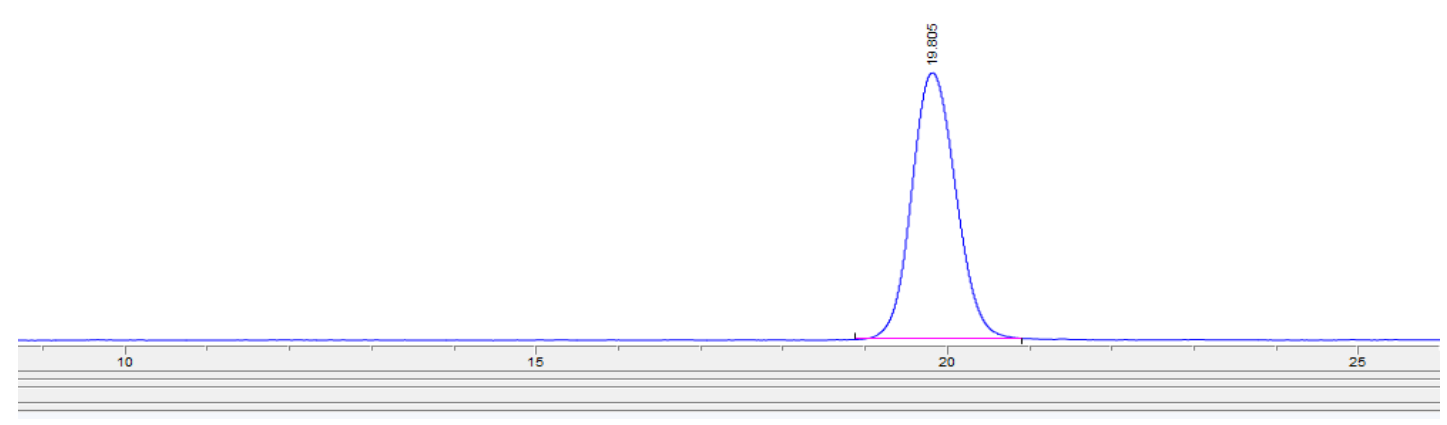

\begin{tabular}{|c|c|l|c|c|c|c|c|}
$\#$ & Time & \multicolumn{1}{c}{ Type } & \multicolumn{1}{c}{ Area } & Height & \multicolumn{1}{c}{ Width } & \multicolumn{1}{c}{ Area\% } & Symmetry \\
\hline 1 & 19.805 & VVR & 31336.3 & 838.6 & 0.4405 & 100.000 & 0.847 \\
\hline
\end{tabular}


HPLC data for product 9 (see procedure):
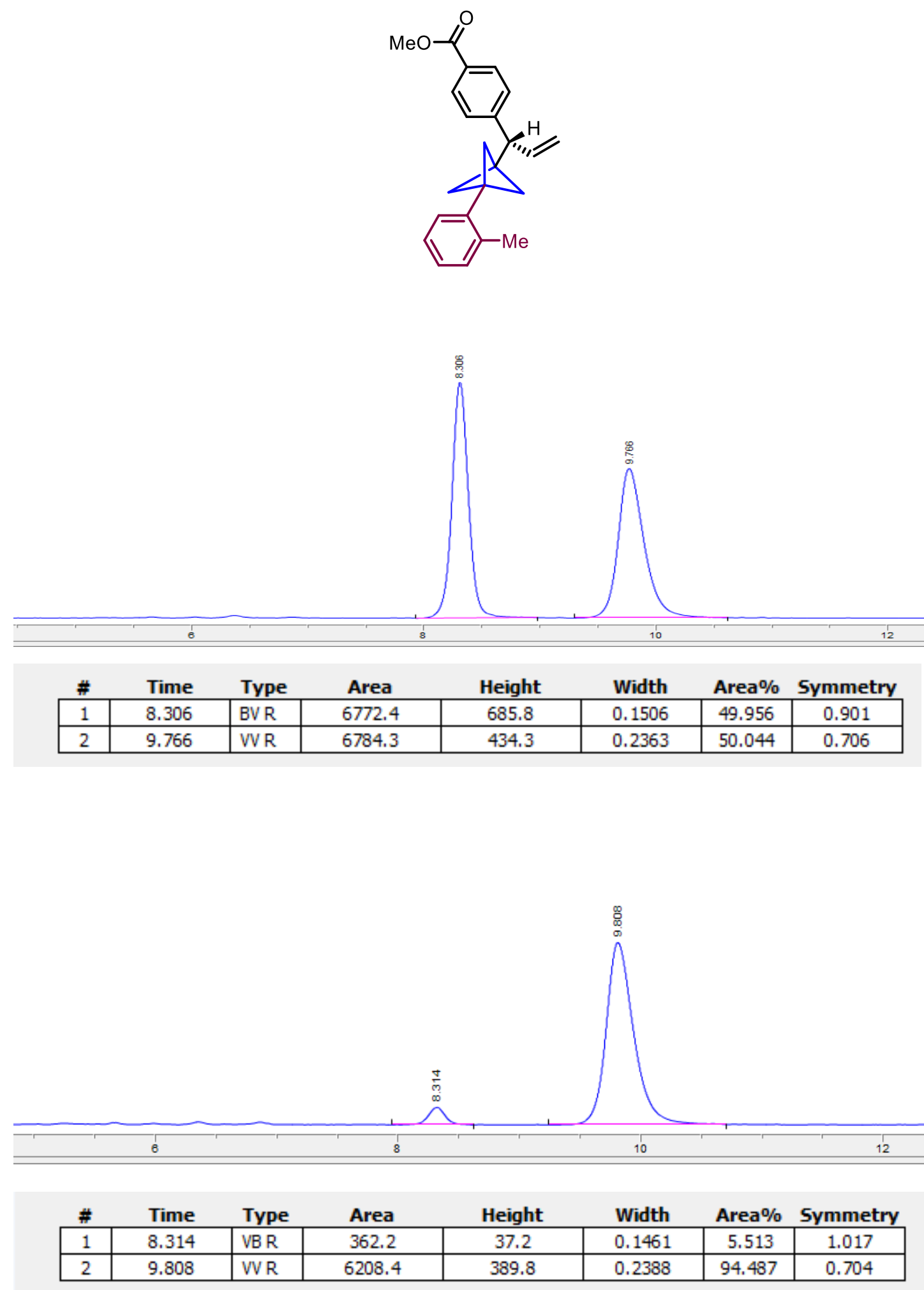
HPLC data for product $\mathbf{1 0}$ (see procedure):
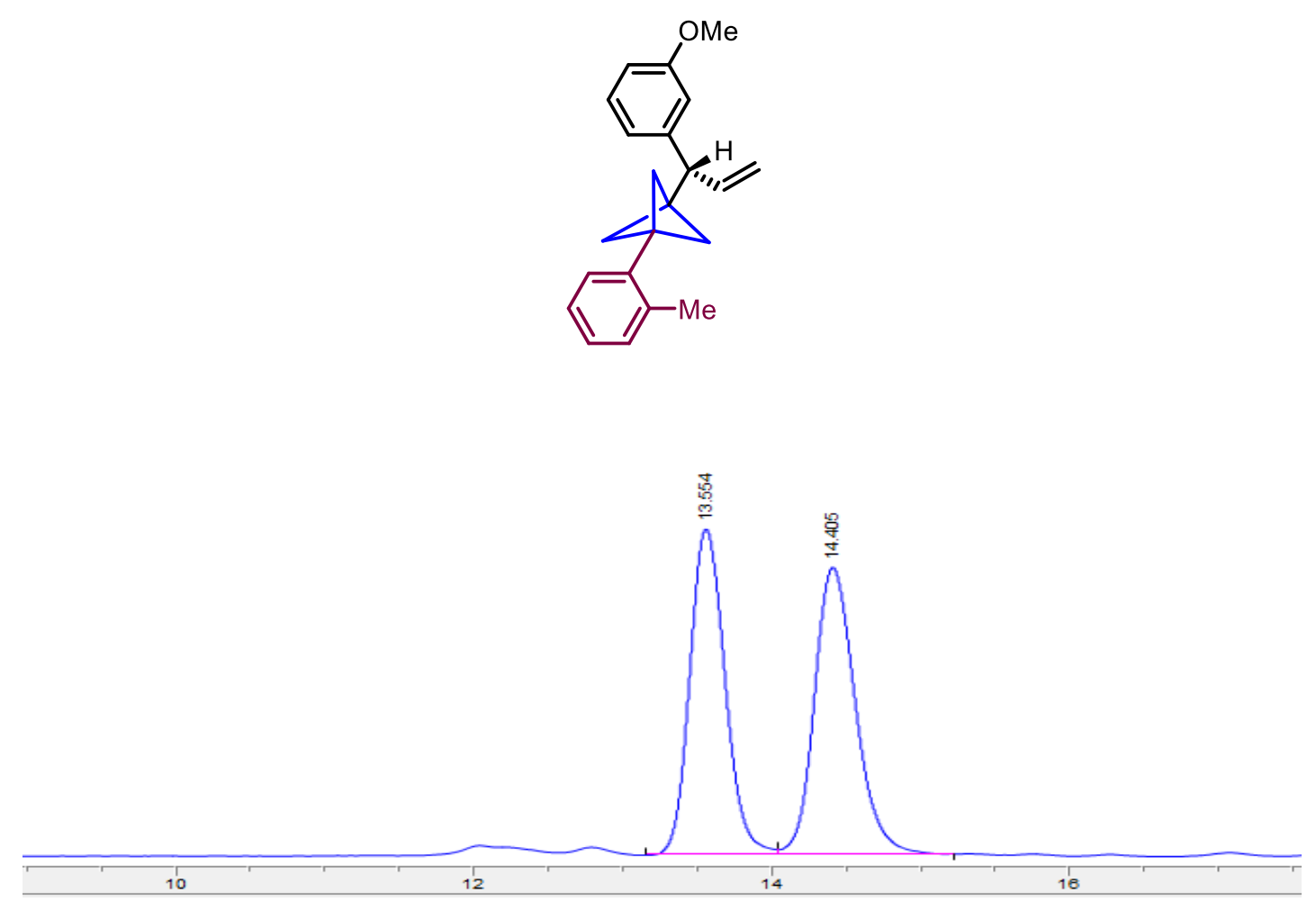

\begin{tabular}{|c|c|c|c|c|c|c|c|}
\hline$\#$ & Time & Type & Area & Height & Width & Area\% & Symmetry \\
\hline 1 & 13.554 & BV & 10403.2 & 631.4 & 0.2362 & 49.516 & 0.821 \\
\hline 2 & 14.405 & VVR & 10606.5 & 556.4 & 0.2954 & 50.484 & 0.769 \\
\hline
\end{tabular}

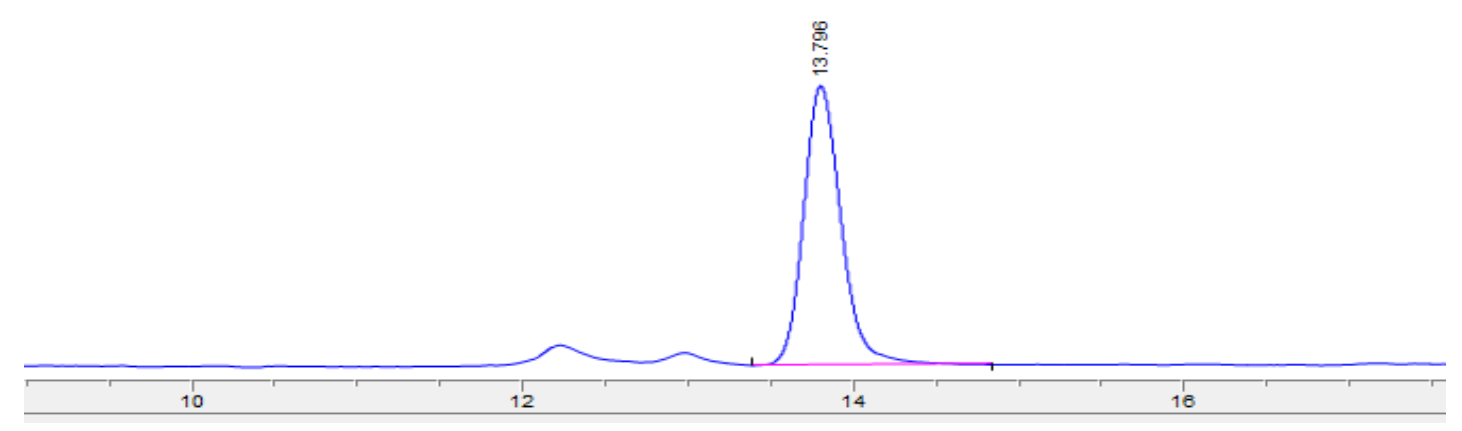

\begin{tabular}{|c|c|c|c|c|c|c|c|}
\multicolumn{1}{c}{ \# } & Time & \multicolumn{1}{c}{ Type } & \multicolumn{1}{c}{ Area } & Height & Width & \multicolumn{2}{c}{ Area\% $\%$ Symmetry } \\
\hline 1 & 13.796 & BVR & 7082.5 & 446.5 & 0.2291 & 100.000 & 0.787 \\
\hline
\end{tabular}


HPLC data for product 11 (see procedure):
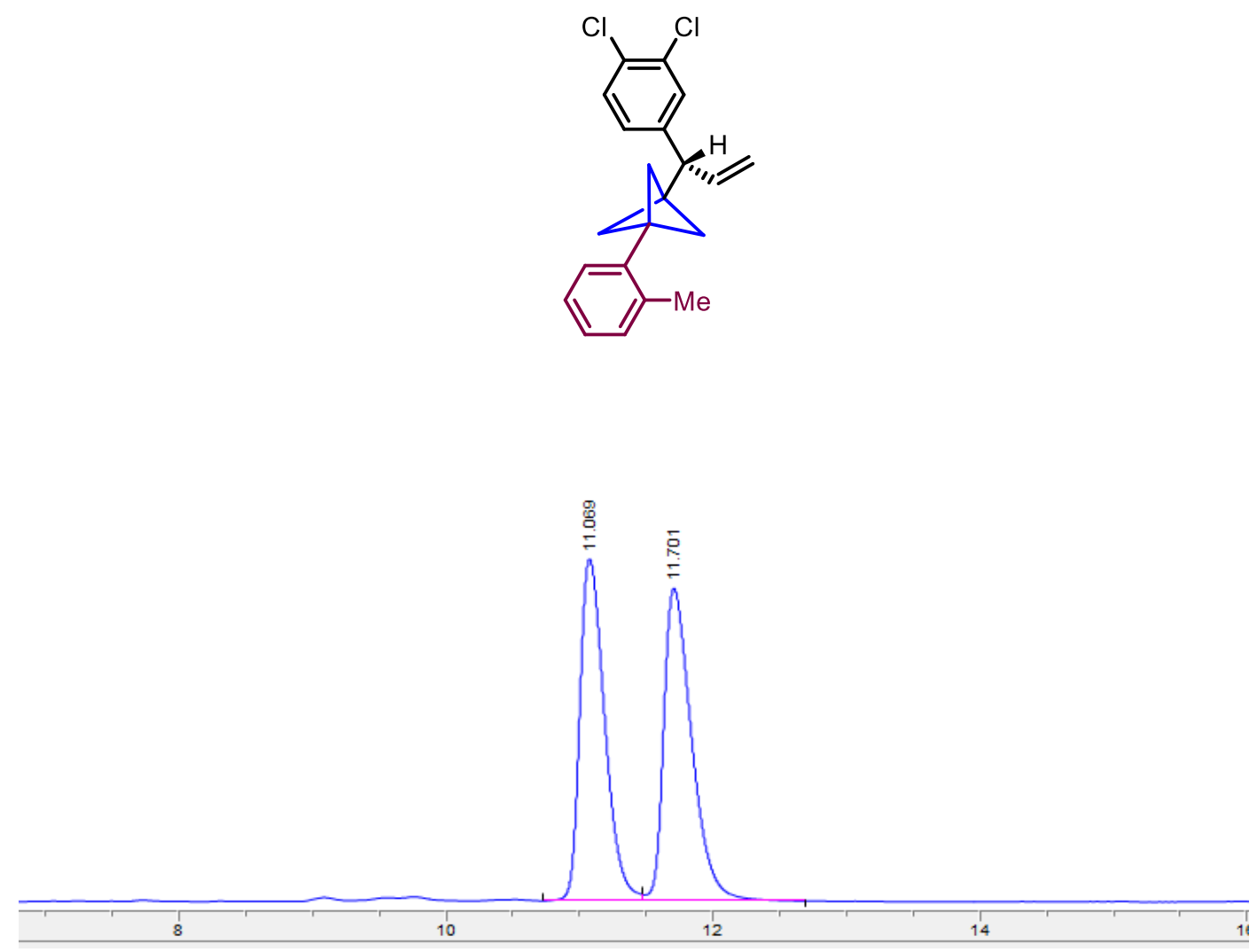

\begin{tabular}{|c|c|l|c|c|c|c|c|}
$\#$ & Time & \multicolumn{1}{c}{ Type } & \multicolumn{1}{c}{ Area } & Height & \multicolumn{1}{c}{ Width } & \multicolumn{2}{c}{ Area\% $\%$ Symmetry } \\
\hline 1 & 11.069 & BV & 9196.9 & 710.7 & 0.1985 & 49.250 & 0.604 \\
\hline 2 & 11.701 & VVR & 9477 & 649.5 & 0.2207 & 50.750 & 0.532 \\
\hline
\end{tabular}

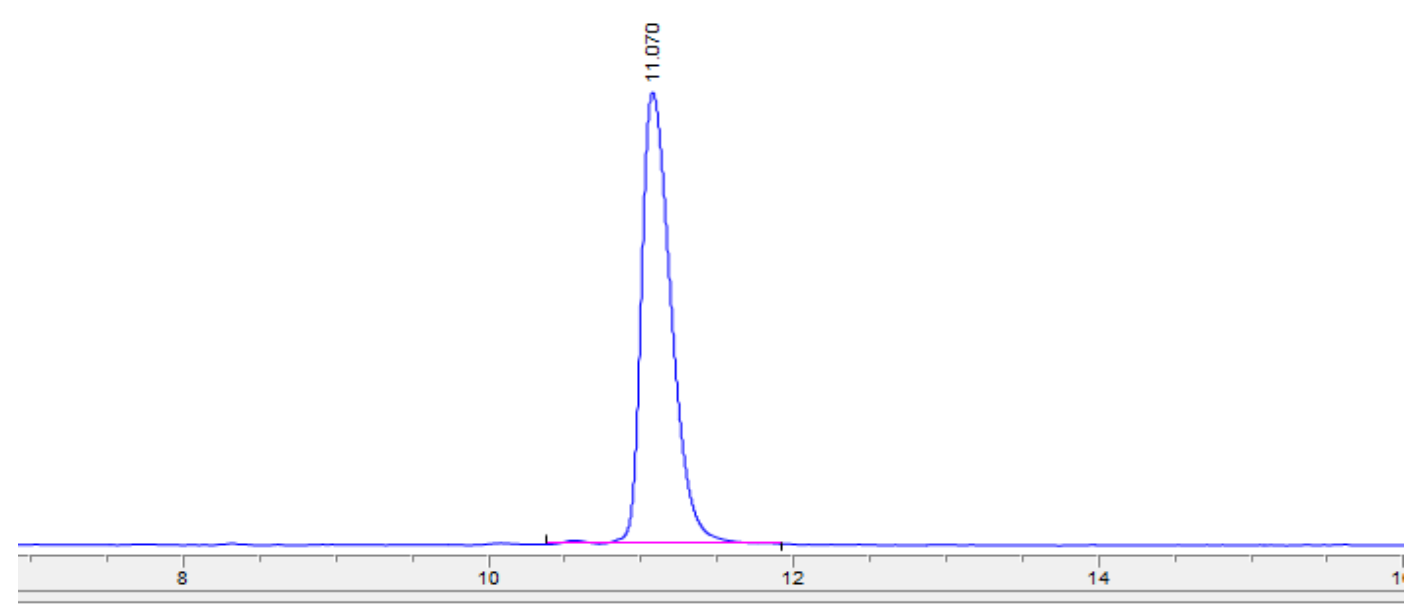

\begin{tabular}{|c|c|l|c|c|c|c|c|}
\multicolumn{2}{l}{ Time } & \multicolumn{1}{c}{ Type } & \multicolumn{1}{c}{ Area } & \multicolumn{1}{c}{ Height } & Width & \multicolumn{2}{c}{ Area\% $\%$ Symmetry } \\
\hline 1 & 11.07 & VVR & 10992.7 & 803.2 & 0.2106 & 100.000 & 0.595 \\
\hline
\end{tabular}


HPLC data for product 12 (see procedure):
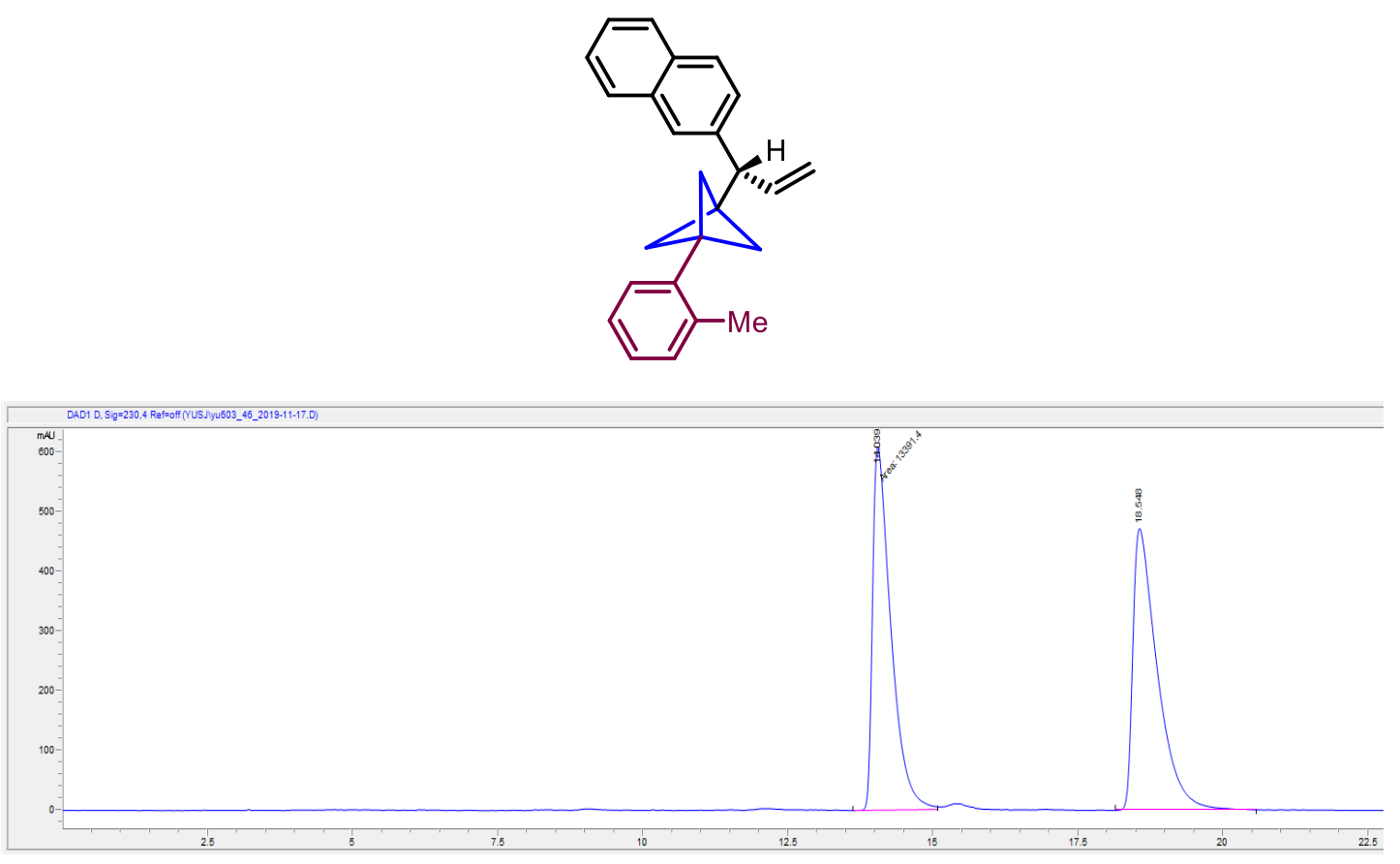

\begin{tabular}{|c|c|l|c|c|c|c|c|} 
\# & \multicolumn{1}{c}{ Time } & \multicolumn{1}{c}{ Type } & \multicolumn{1}{c}{ Area } & Height & \multicolumn{2}{c}{ Width } & \multicolumn{2}{c|}{ Area\% } & Symmetry \\
\hline 1 & 14.039 & MF & 13391.4 & 606.7 & 0.3679 & 49.199 & 0.392 \\
\hline 2 & 18.548 & BV R & 13827.7 & 470.5 & 0.4289 & 50.801 & 0.35 \\
\hline
\end{tabular}

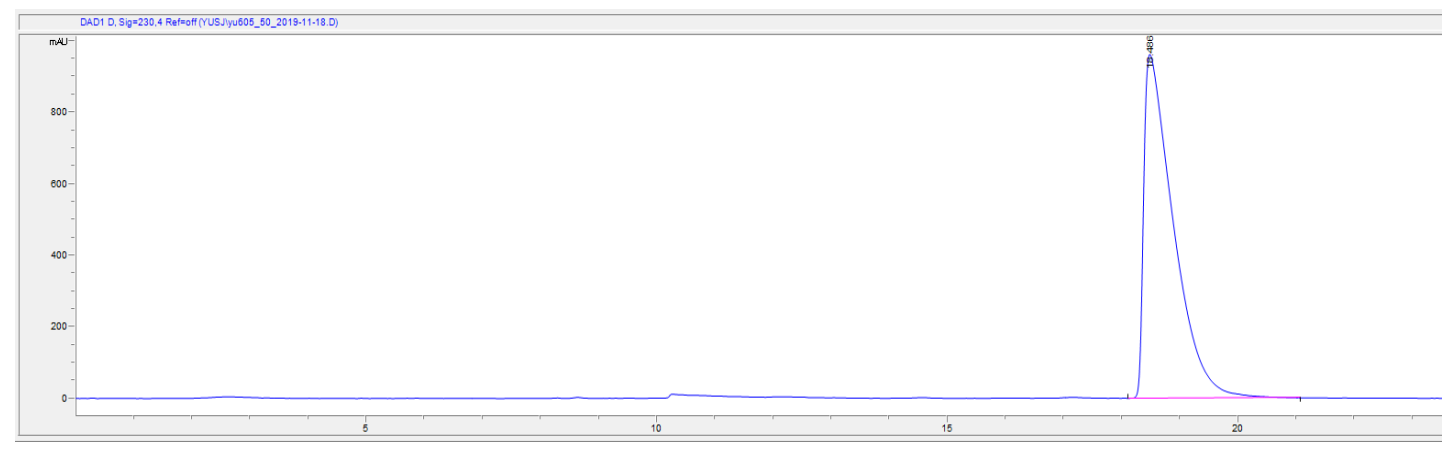

\begin{tabular}{|c|c|l|c|c|c|c|c|}
$\#$ & Time & \multicolumn{1}{c}{ Type } & Area & \multicolumn{1}{c}{ Height } & \multicolumn{2}{c}{ Width } & \multicolumn{2}{c|}{ Area\% $\%$ Symmetry } \\
\hline 1 & 18.486 & BVR & 34103 & 963.9 & 0.5168 & 100.000 & 0.236 \\
\hline
\end{tabular}


HPLC data for product 13a (see procedure):
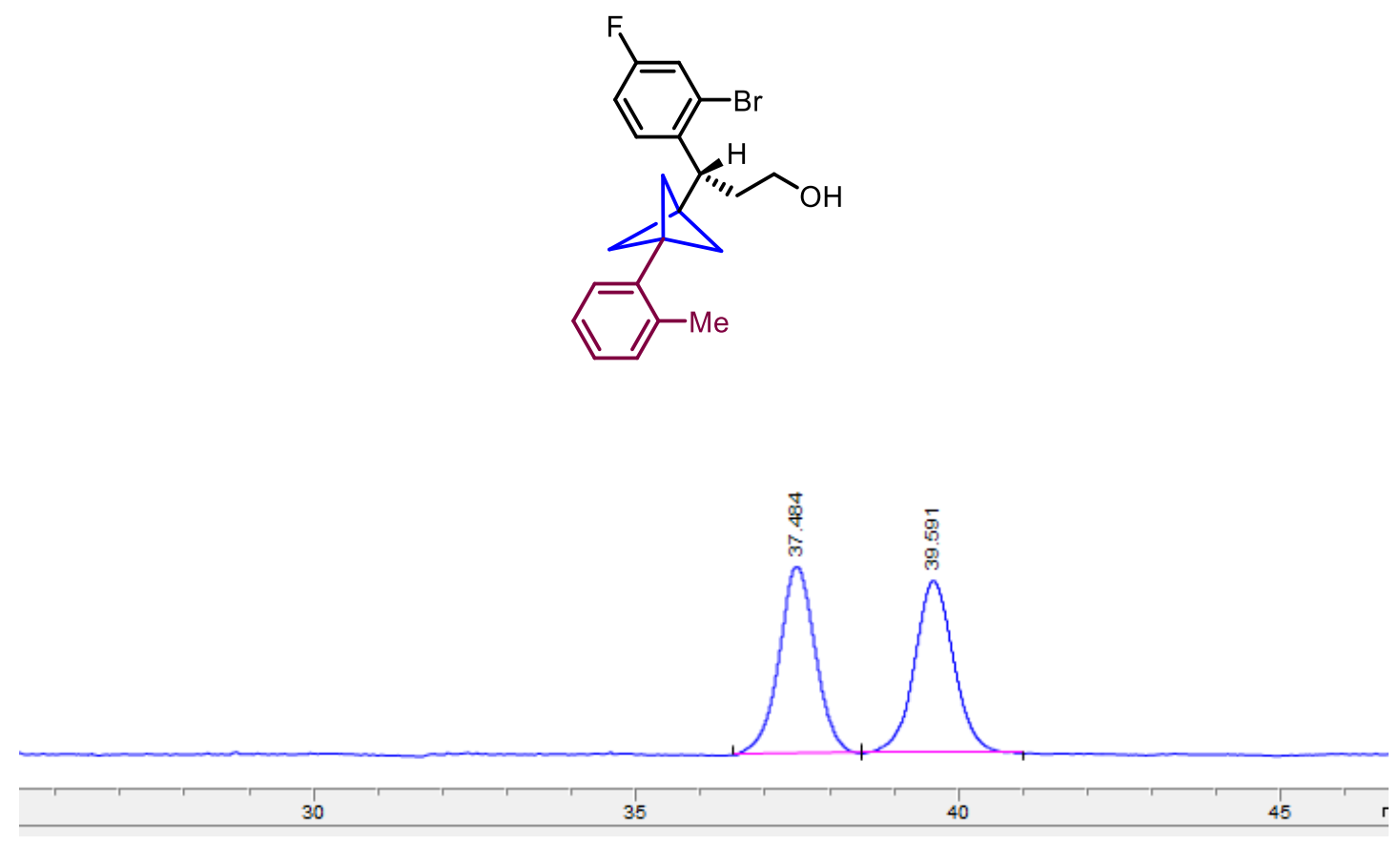

\begin{tabular}{|c|c|l|c|c|c|c|c|} 
\# & Time & \multicolumn{1}{c}{ Type } & Area & Height & \multicolumn{1}{c}{ Width } & \multicolumn{2}{c|}{ Area\% $\%$ Symmetry } \\
\hline 1 & 37.484 & BB & 4389 & 110.6 & 0.4998 & 50.290 & 1.015 \\
\hline 2 & 39.591 & BV R & 4338.5 & 101.9 & 0.5332 & 49.710 & 0.888 \\
\hline
\end{tabular}

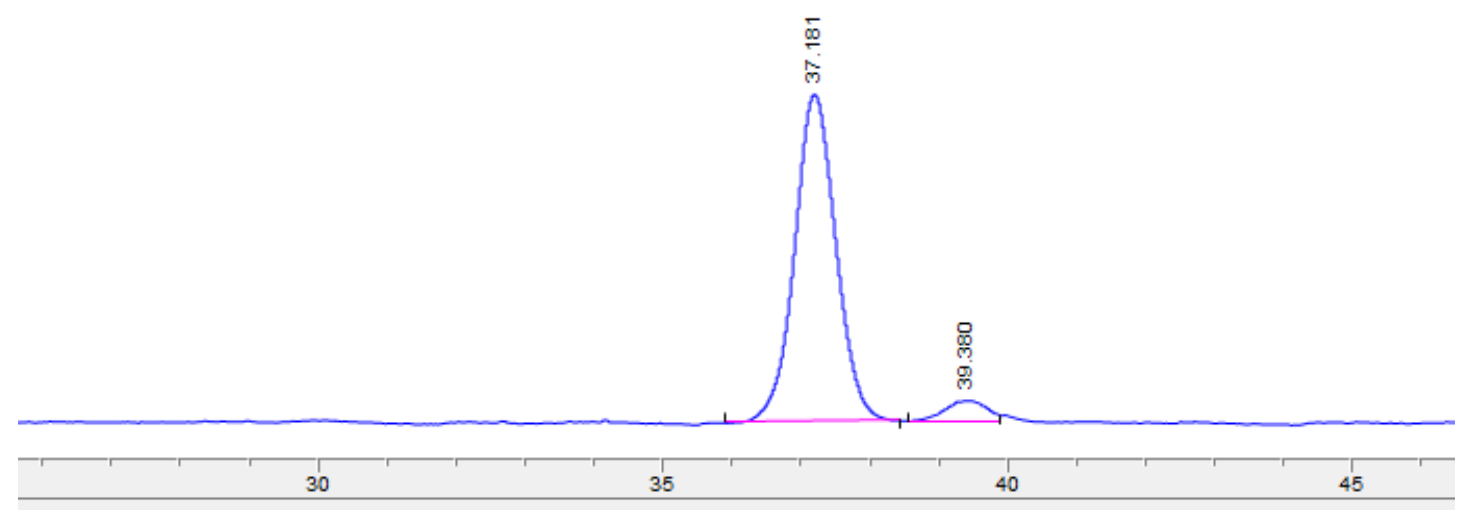

\begin{tabular}{|c|c|c|c|c|c|c|c|}
\hline$\#$ & Time & Type & Area & Height & Width & Area $\%$ & Symmetry \\
\hline 1 & 37.181 & VVR & 7576.4 & 180.3 & 0.6063 & 93.536 & 0.923 \\
\hline 2 & 39.38 & BV & 523.6 & 11.7 & 0.5267 & 6.464 & 1.068 \\
\hline
\end{tabular}


HPLC data for product 14 (see procedure):
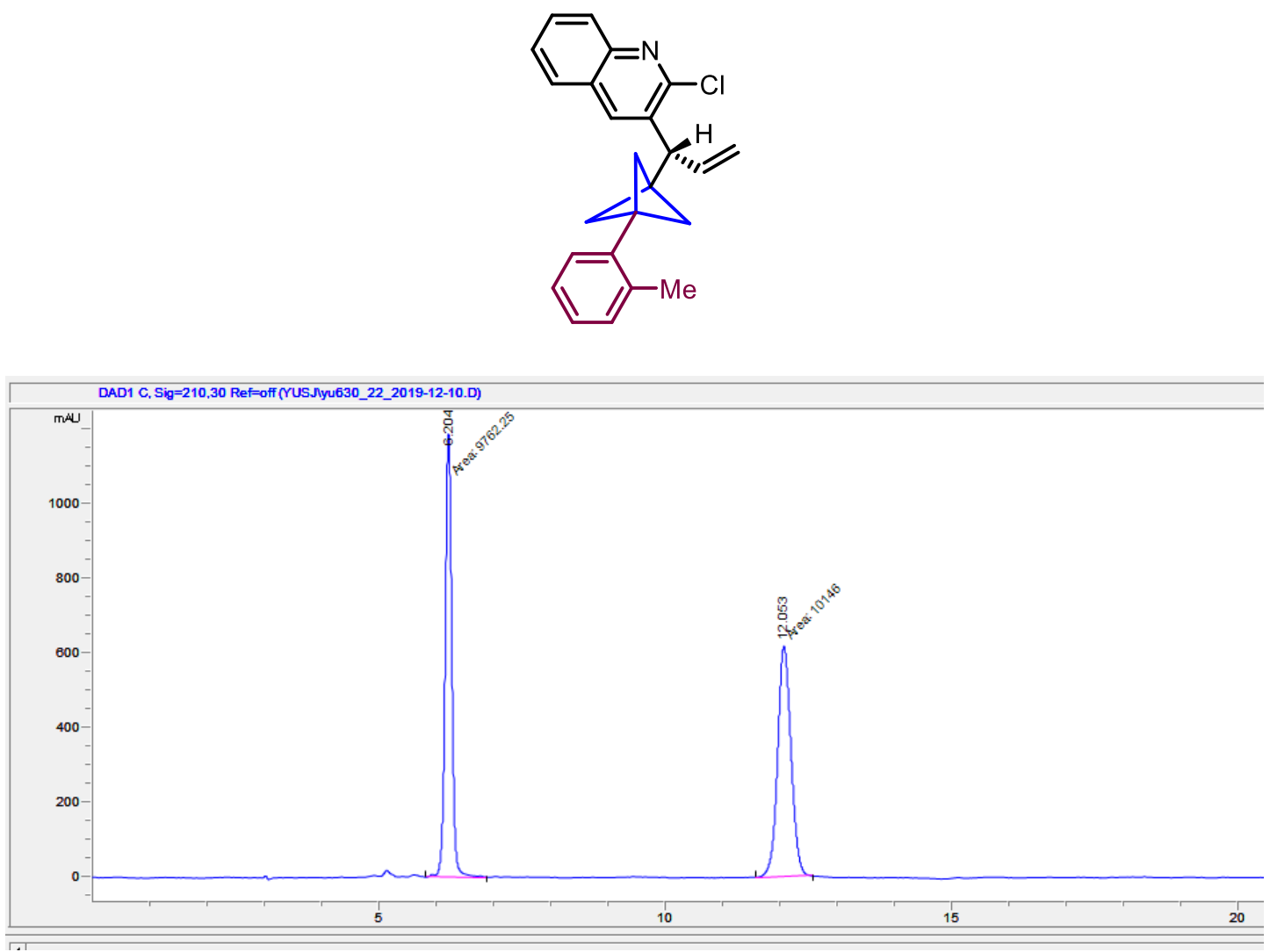

\begin{tabular}{|c|c|l|c|c|c|c|c|} 
\# & \multicolumn{1}{c}{ Time } & \multicolumn{1}{c}{ Type } & \multicolumn{1}{c}{ Area } & Height & Width & \multicolumn{2}{c}{ Area\% $\%$ Symmetry } \\
\hline 1 & 6.204 & MM & 9762.2 & 1190.6 & 0.1367 & 49.036 & 0.934 \\
\hline 2 & 12.053 & MM & 10146 & 619.1 & 0.2732 & 50.964 & 0.891 \\
\hline
\end{tabular}

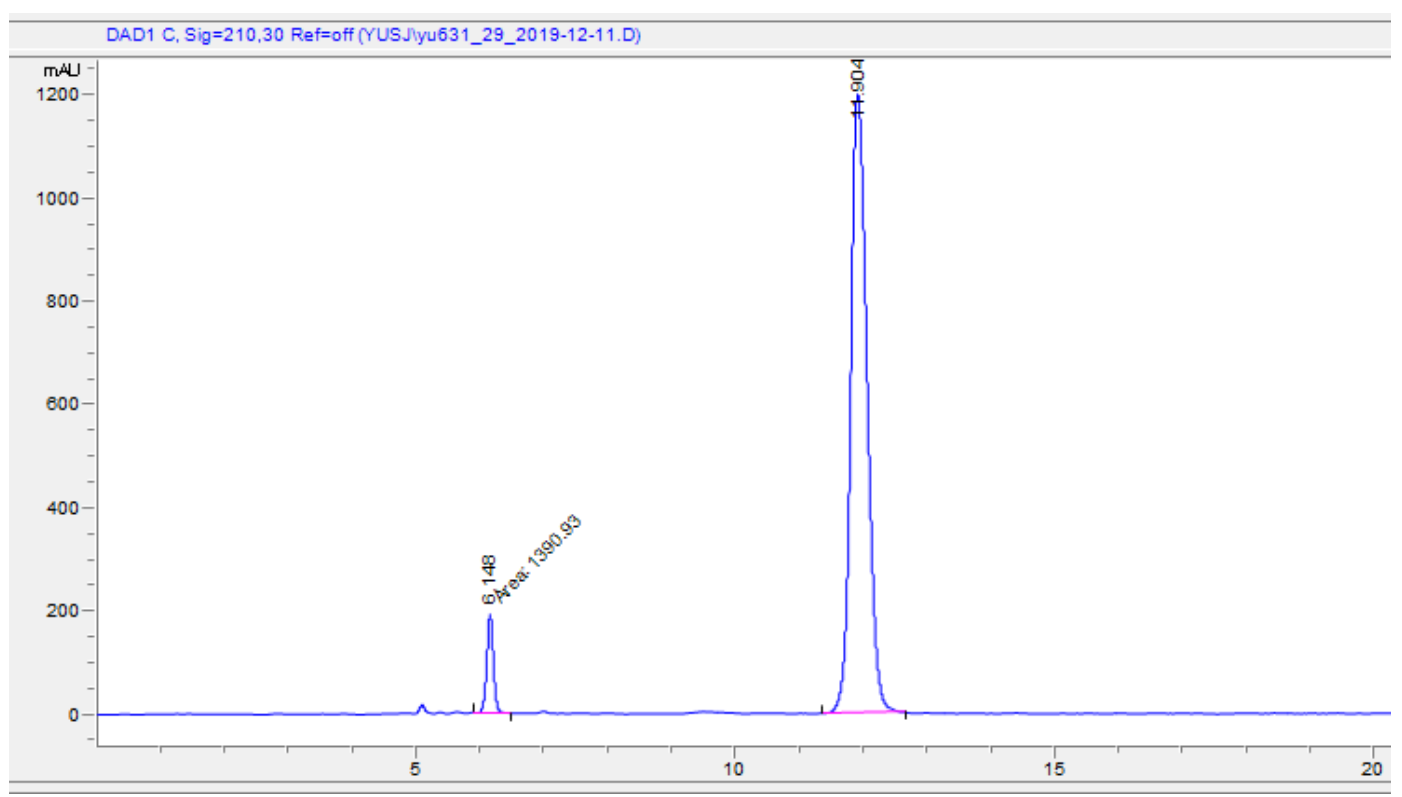

\begin{tabular}{|c|c|c|c|c|c|c|c|}
\hline$\#$ & Time & Type & Area & Height & Width & Area $\%$ & Symmetry \\
\hline 1 & 6.148 & FM & 1390.9 & 192.7 & 0.1203 & 6.136 & 0.991 \\
\hline 2 & 11.904 & VVR & 21278.4 & 1203.5 & 0.2638 & 93.864 & 0.704 \\
\hline
\end{tabular}


HPLC data for product 15 (see procedure):
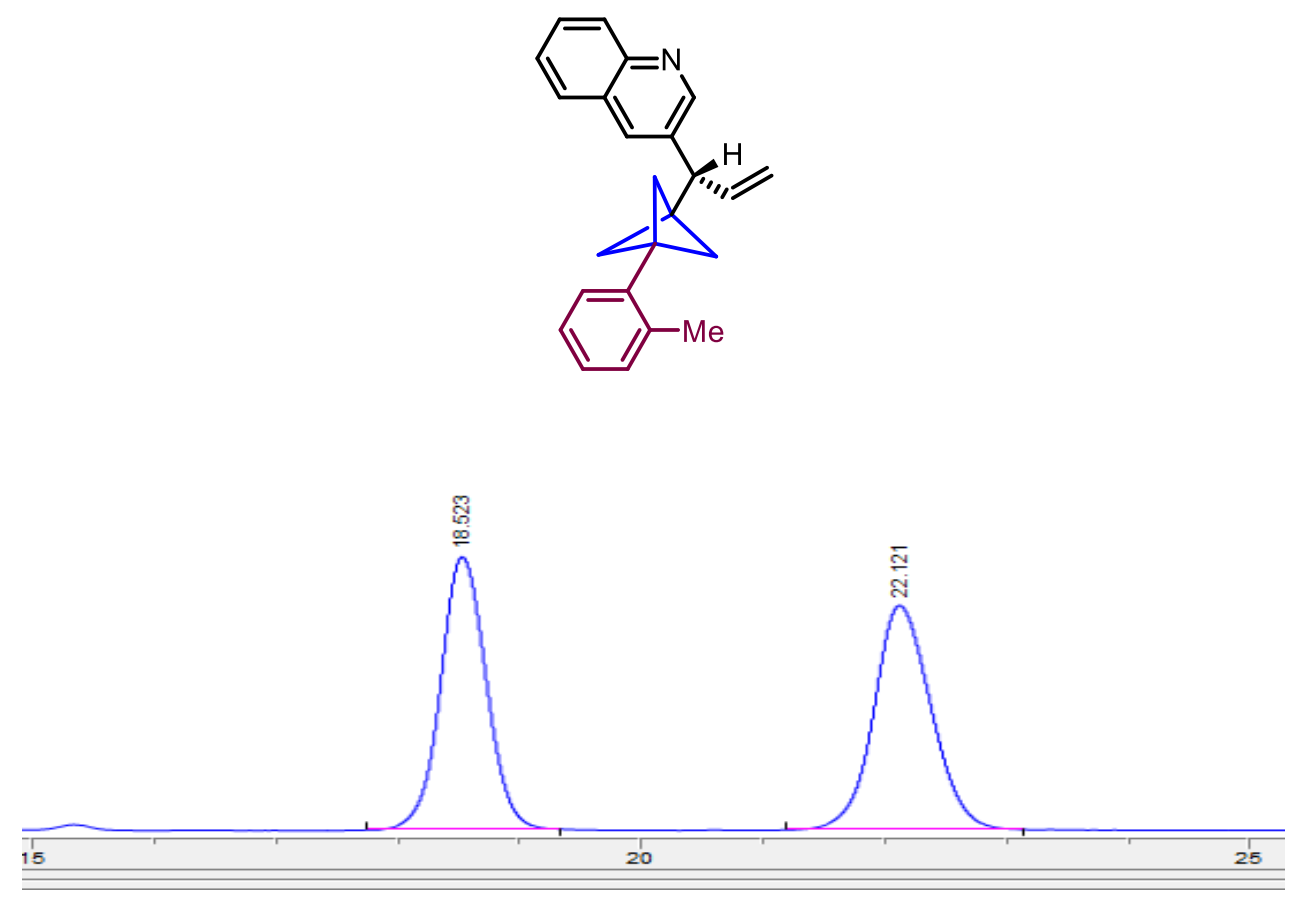

\begin{tabular}{|c|c|l|c|c|c|c|c|} 
\# & \multicolumn{1}{c}{ Time } & \multicolumn{1}{c}{ Type } & \multicolumn{1}{c}{ Area } & Height & \multicolumn{1}{c}{ Width } & \multicolumn{1}{c}{ Area\% } & Symmetry \\
\hline 1 & 18.523 & VV R & 25626.2 & 981.3 & 0.3432 & 49.158 & 0.834 \\
\hline 2 & 22.121 & VVR & 26503.8 & 807 & 0.3892 & 50.842 & 0.817 \\
\hline
\end{tabular}

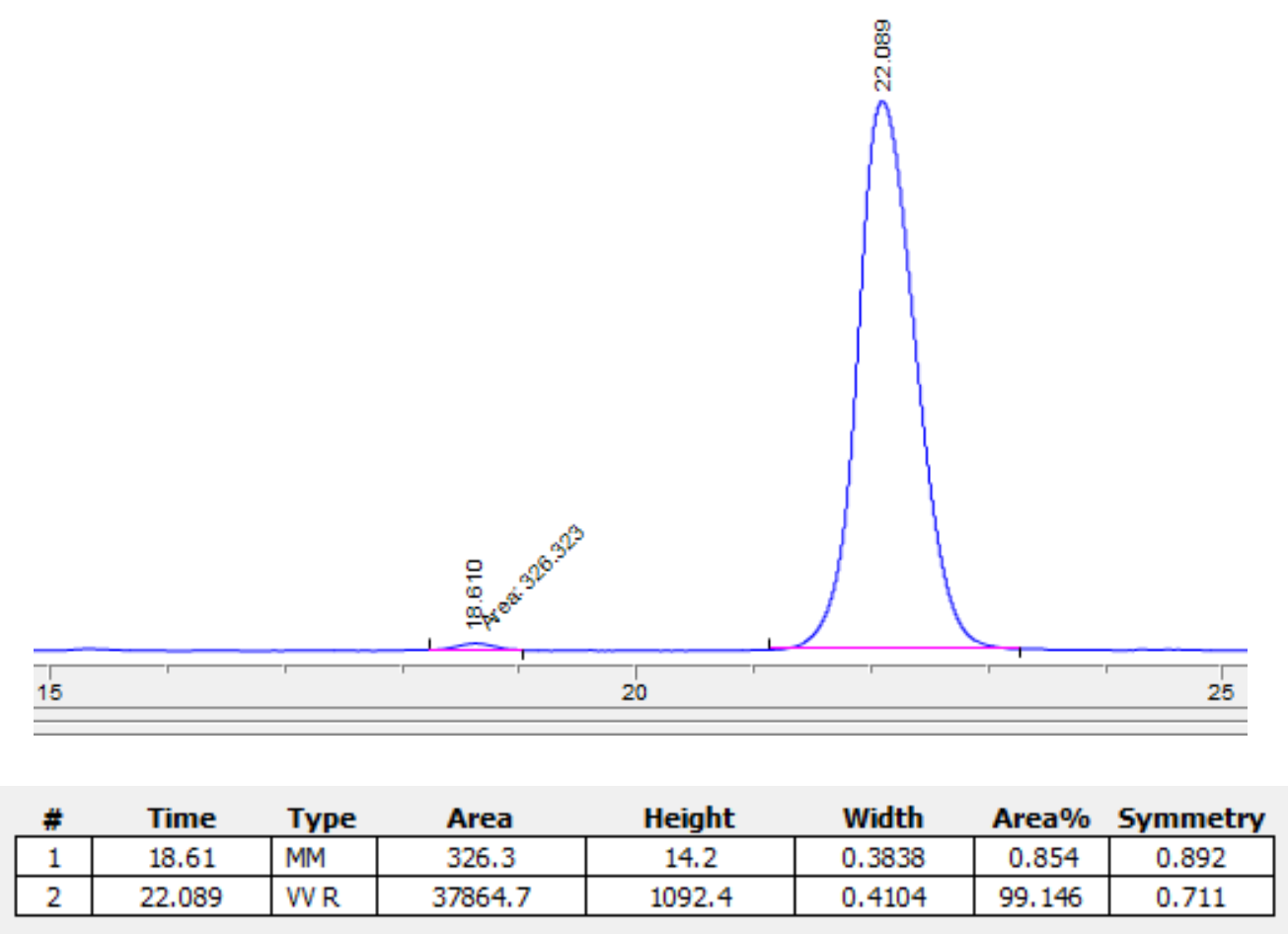


HPLC data for product $\mathbf{1 6}$ (see procedure):
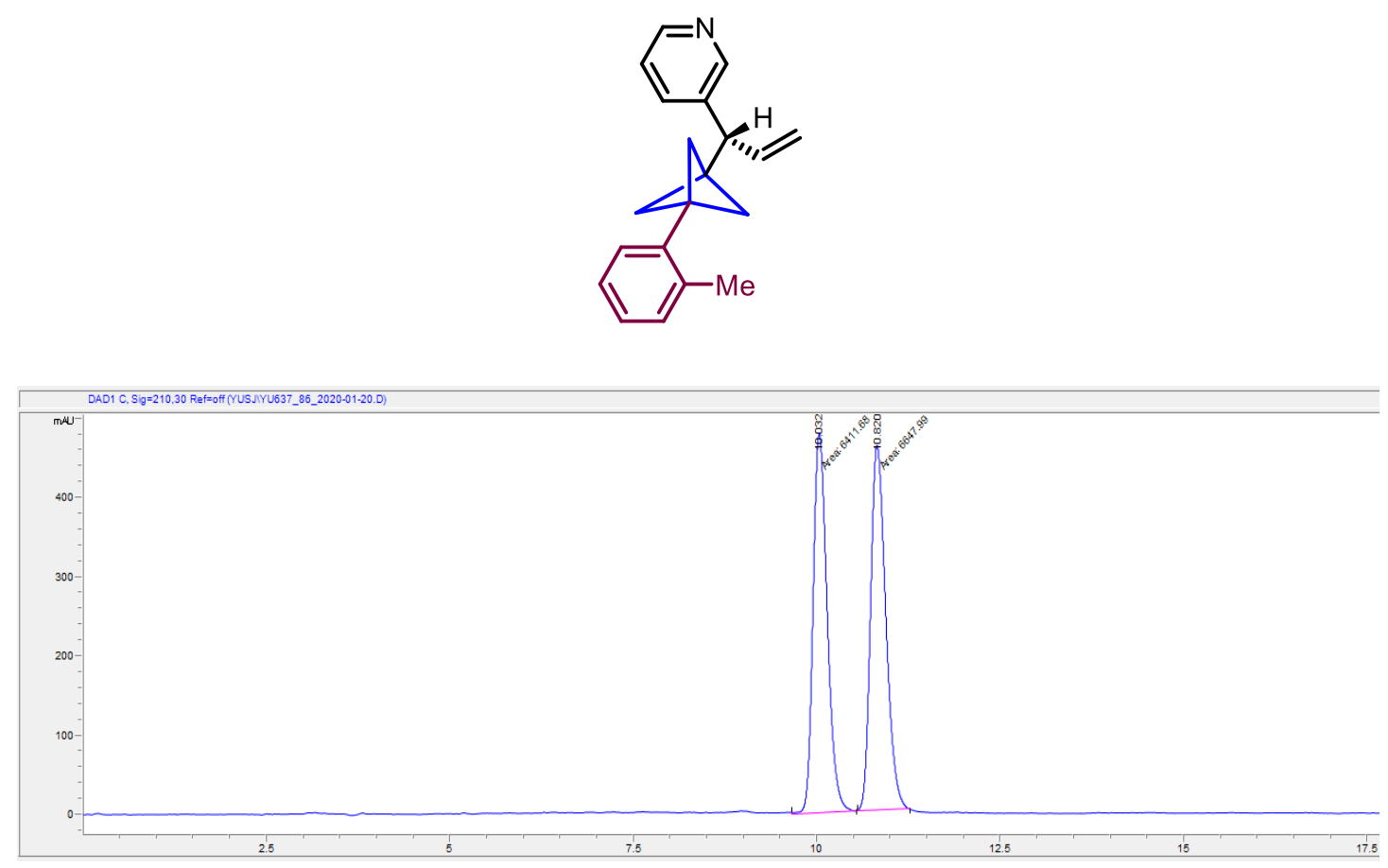

\begin{tabular}{|c|c|l|c|c|c|c|c|} 
\# & \multicolumn{1}{c}{ Time } & \multicolumn{1}{c}{ Type } & \multicolumn{1}{c}{ Area } & \multicolumn{1}{c}{ Height } & \multicolumn{2}{c|}{ Width } & \multicolumn{2}{c}{ Area\% $\%$ Symmetry } \\
\hline 1 & 10.032 & MM & 6411.7 & 478.5 & 0.2233 & 49.095 & 0.743 \\
\hline 2 & 10.82 & MM & 6648 & 460.4 & 0.2407 & 50.905 & 0.719 \\
\hline
\end{tabular}

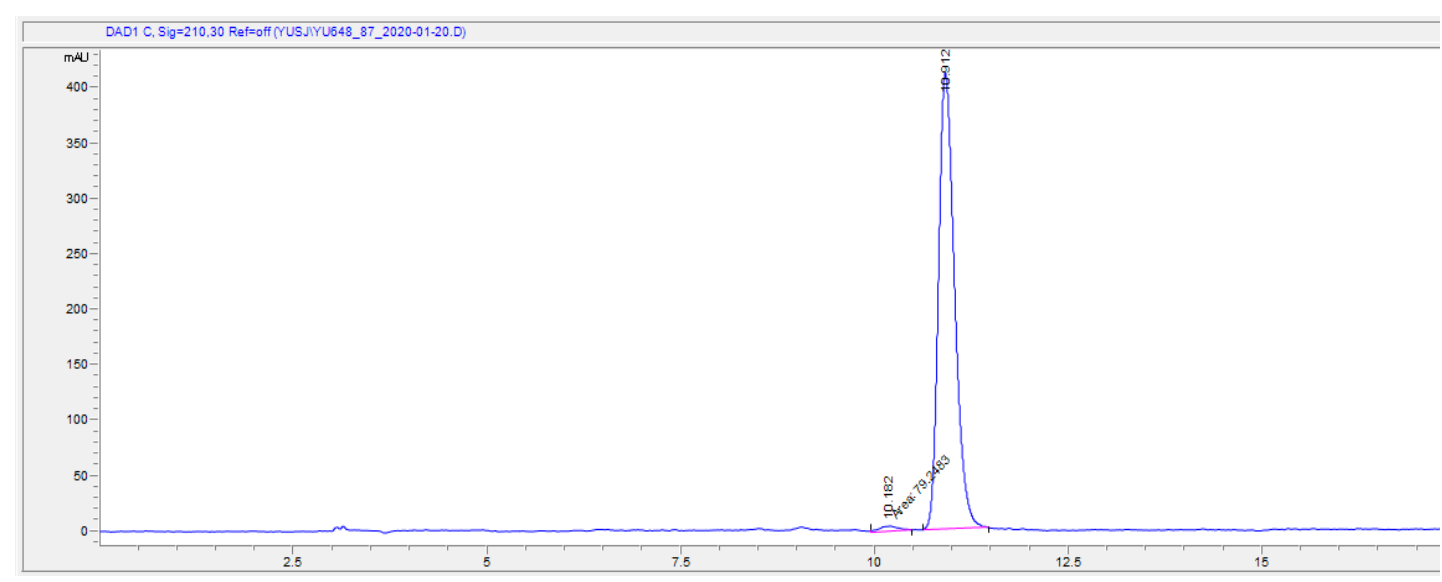

\begin{tabular}{|c|c|l|c|c|c|c|c|} 
\# & Time & \multicolumn{1}{c}{ Type } & Area & Height & \multicolumn{1}{c}{ Width } & \multicolumn{2}{c|}{ Area\% } \\
\hline 1 & 10.182 & MM & 79.2 & 4.7 & 0.2799 & 1.312 & 1.208 \\
\hline 2 & 10.912 & BV R & 5959.3 & 411.4 & 0.2085 & 98.688 & 0.706 \\
\hline
\end{tabular}


HPLC data for product 17 (see procedure):
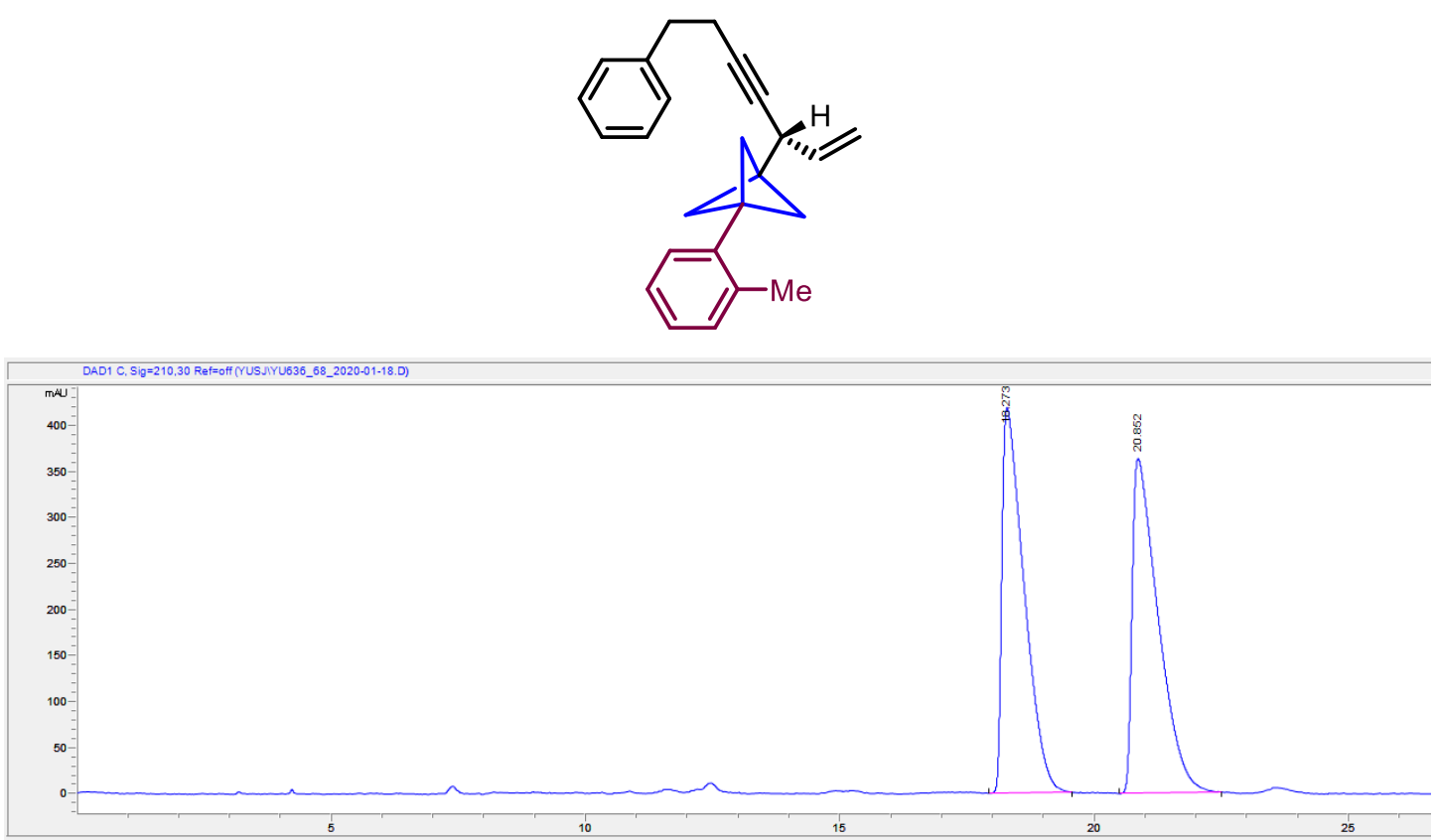

\begin{tabular}{|c|c|l|c|c|c|c|c|}
$\#$ & \multicolumn{1}{c}{ Time } & \multicolumn{1}{c}{ Type } & \multicolumn{1}{c}{ Area } & \multicolumn{1}{c}{ Height } & \multicolumn{1}{c}{ Width } & \multicolumn{2}{c|}{ Area\% $\%$ Symmetry } \\
\hline 1 & 18.273 & BV R & 12691.4 & 419.9 & 0.3991 & 49.495 & 0.276 \\
\hline 2 & 20.852 & BV R & 12950.4 & 364.6 & 0.458 & 50.505 & 0.273 \\
\hline
\end{tabular}

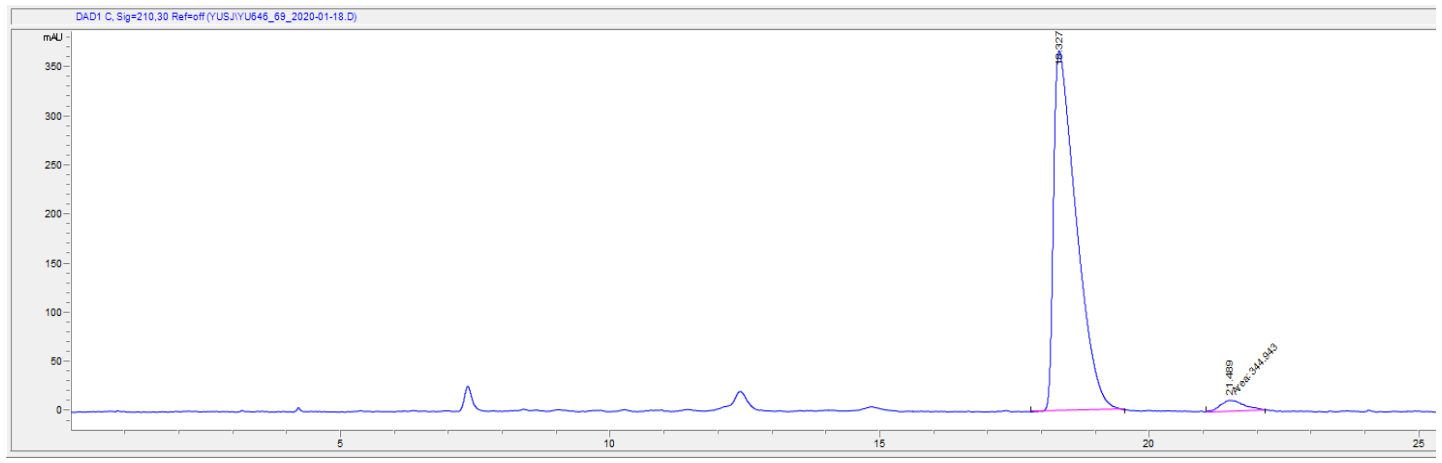

\begin{tabular}{|c|c|l|c|c|c|c|c|} 
\# & Time & \multicolumn{1}{c}{ Type } & Area & Height & \multicolumn{1}{c}{ Width } & \multicolumn{2}{c}{ Area\% $\%$ Symmetry } \\
\hline 1 & 18.327 & VV R & 10764 & 368.1 & 0.3747 & 96.895 & 0.295 \\
\hline 2 & 21.489 & MM & 344.9 & 10.9 & 0.527 & 3.105 & 0.674 \\
\hline
\end{tabular}


HPLC data for product $\mathbf{2 0}$ (see procedure):
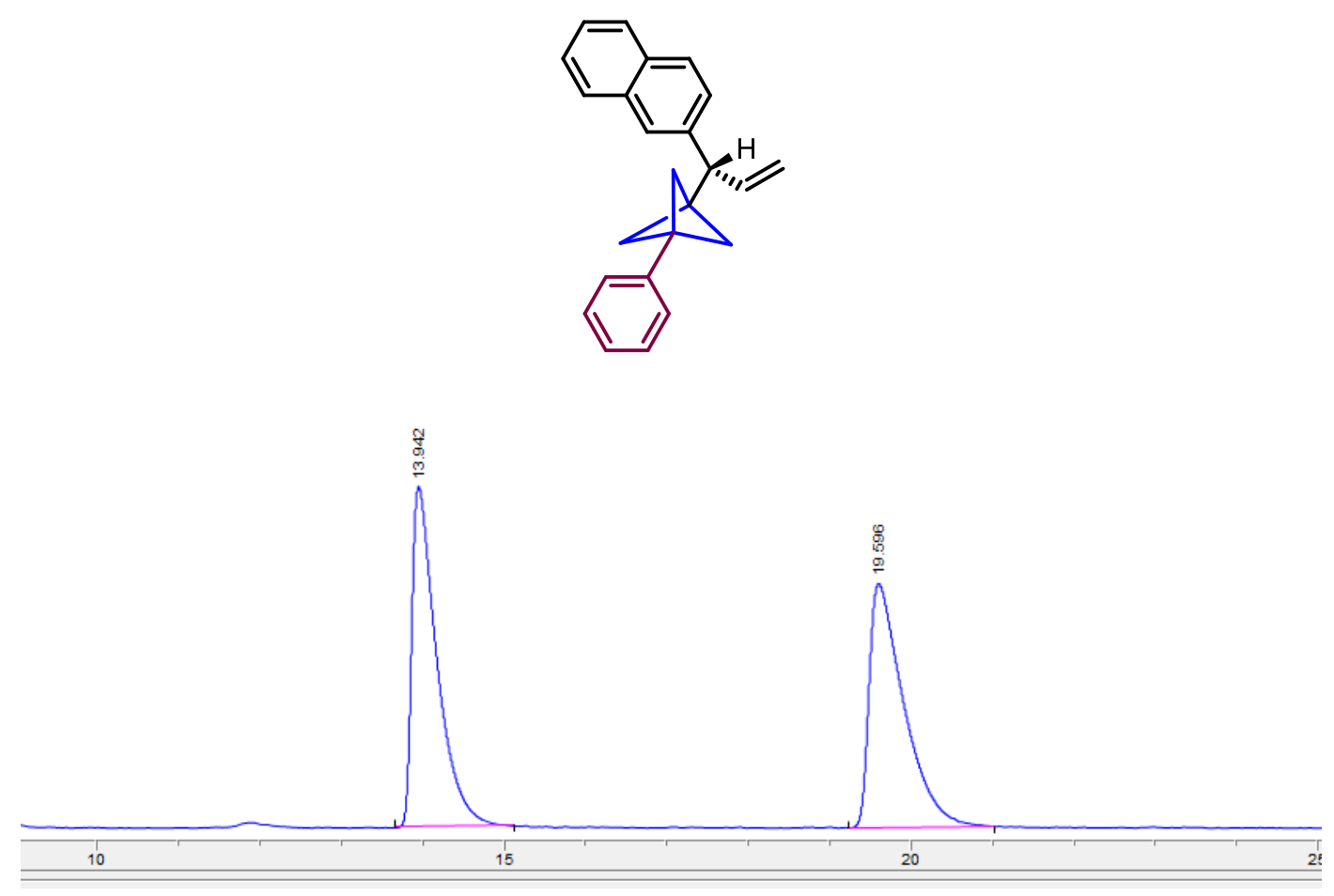

\begin{tabular}{|c|c|l|c|c|c|c|c|} 
\# & \multicolumn{1}{c}{ Time } & \multicolumn{1}{c}{ Type } & \multicolumn{1}{c}{ Area } & Height & \multicolumn{1}{c}{ Width } & \multicolumn{1}{c}{ Area\% $\%$ Symmetry } \\
\hline 1 & 13.942 & BV R & 11653.5 & 541.4 & 0.3081 & 50.284 & 0.346 \\
\hline 2 & 19.596 & BV R & 11522 & 387.9 & 0.3964 & 49.716 & 0.357 \\
\hline
\end{tabular}

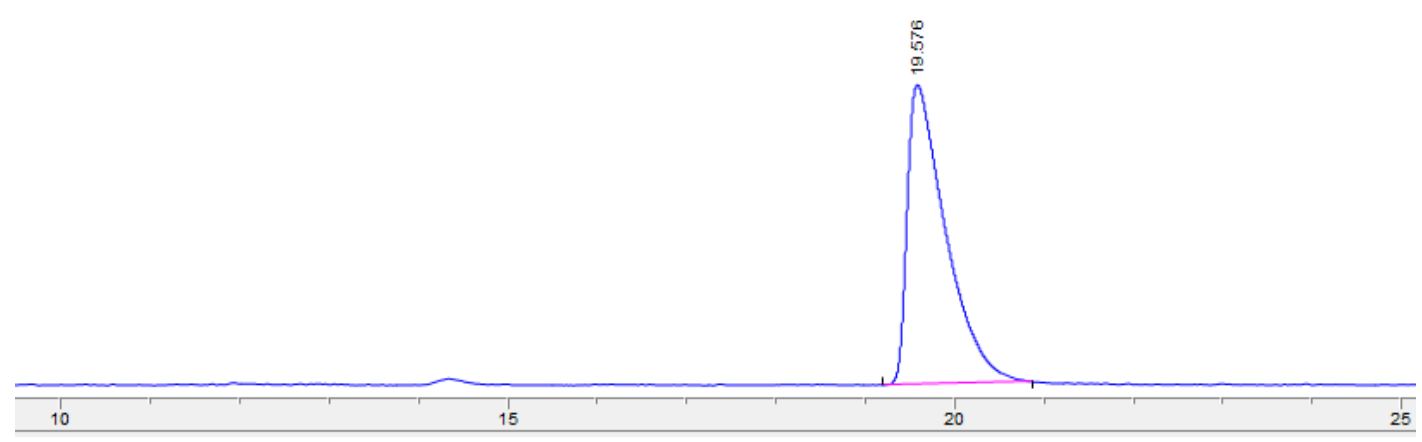

\begin{tabular}{|c|c|c|c|c|c|c|c|}
\hline$\#$ & Time & Type & Area & Height & Width & Area $\%$ & Symmetry \\
\hline 1 & 19.576 & BV R & 13025.1 & 433.5 & 0.409 & 100.000 & 0.338 \\
\hline
\end{tabular}


HPLC data for product 21 (see procedure):
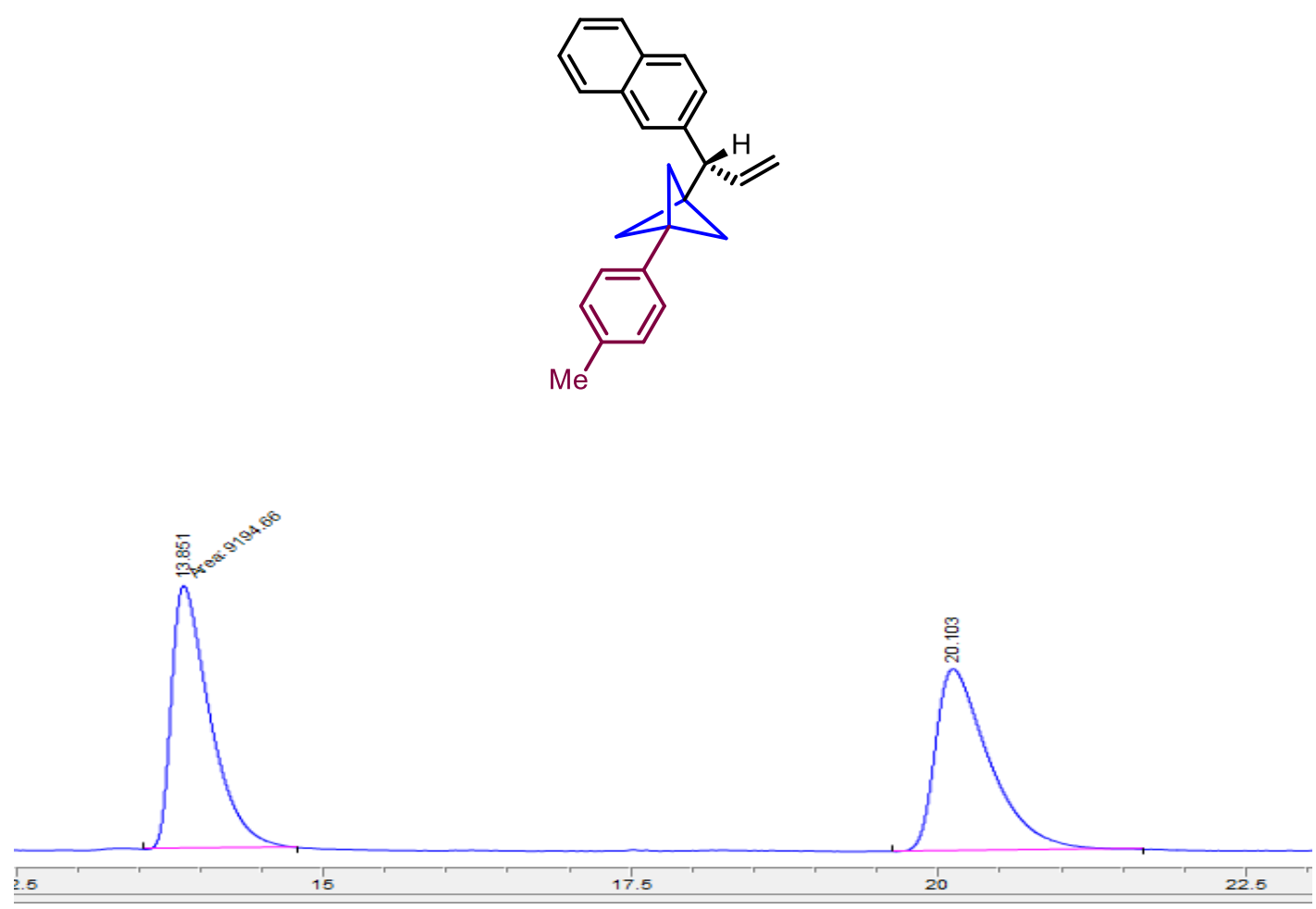

\begin{tabular}{|c|c|l|c|c|c|c|c|}
\multicolumn{1}{c}{ \# } & Time & \multicolumn{1}{c}{ Type } & \multicolumn{1}{c}{ Area } & \multicolumn{1}{c}{ Height } & \multicolumn{1}{c}{ Width } & \multicolumn{2}{c}{ Area\% $\%$ Symmetry } \\
\hline 1 & 13.851 & MM & 9194.7 & 422.2 & 0.363 & 50.600 & 0.452 \\
\hline 2 & 20.103 & BV R & 8976.6 & 292.7 & 0.4298 & 49.400 & 0.45 \\
\hline
\end{tabular}

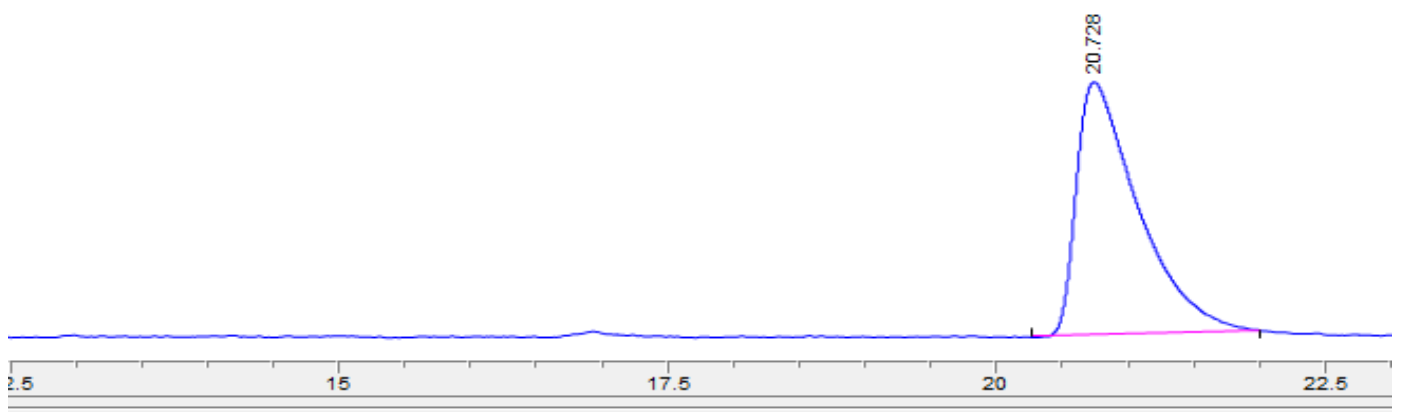

\begin{tabular}{|c|c|l|c|c|c|c|c|}
$\#$ & Time & \multicolumn{1}{c}{ Type } & Area & Height & \multicolumn{1}{c}{ Width } & \multicolumn{1}{c}{ Area\% $\%$ Symmetry } \\
\hline 1 & 20.728 & BV R & 10207.5 & 310.5 & 0.4517 & 100.000 & 0.377 \\
\hline
\end{tabular}


HPLC data for product 22 (see procedure):
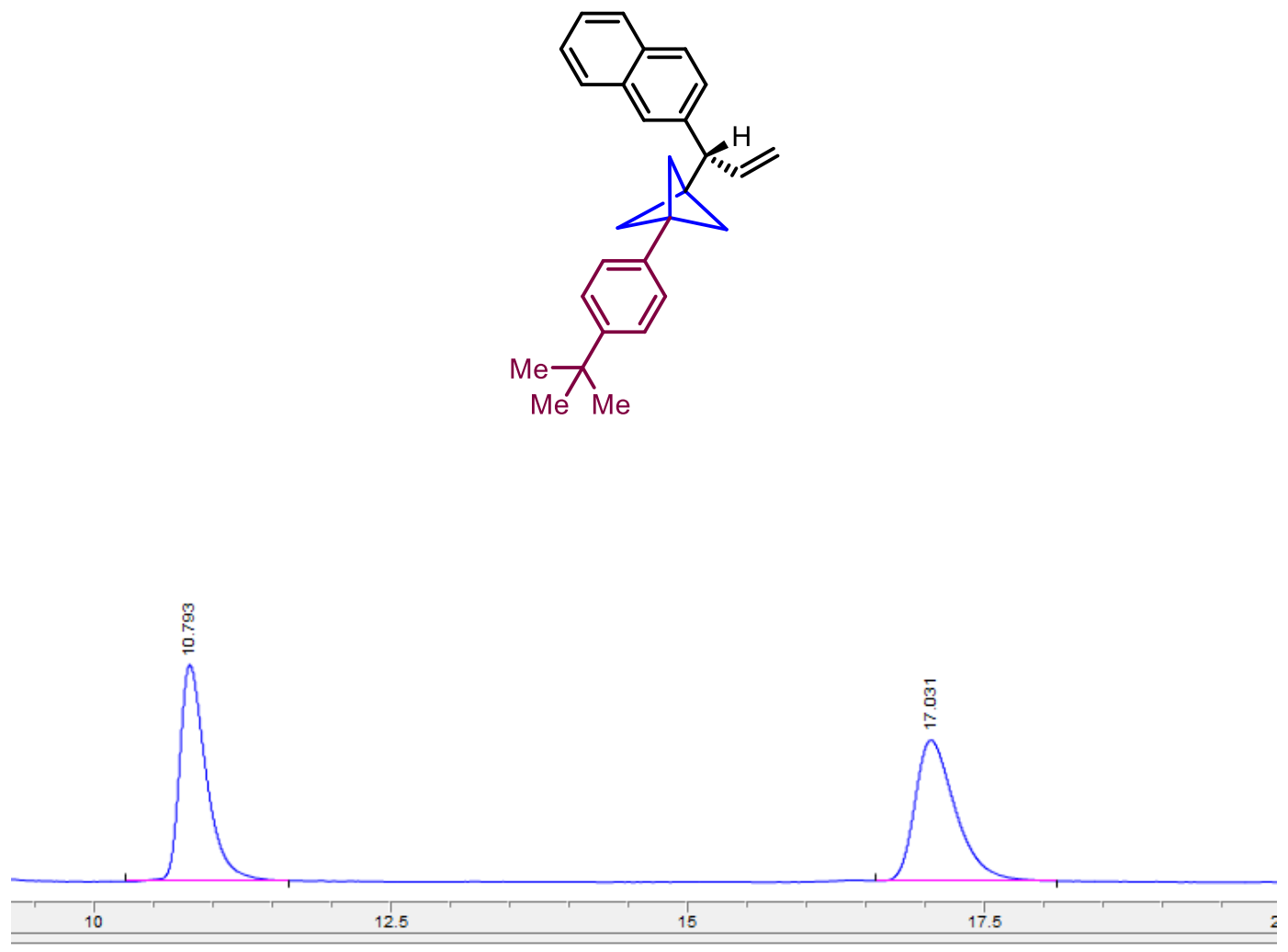

\begin{tabular}{|c|c|c|c|c|c|c|c|}
\hline$\#$ & Time & Type & Area & Height & Width & Area \% & Symmetry \\
\hline 1 & 10.793 & BVR & 4944.2 & 315.4 & 0.2314 & 50.367 & 0.582 \\
\hline 2 & 17.031 & VVR & 4872.2 & 205.3 & 0.3373 & 49.633 & 0.613 \\
\hline
\end{tabular}

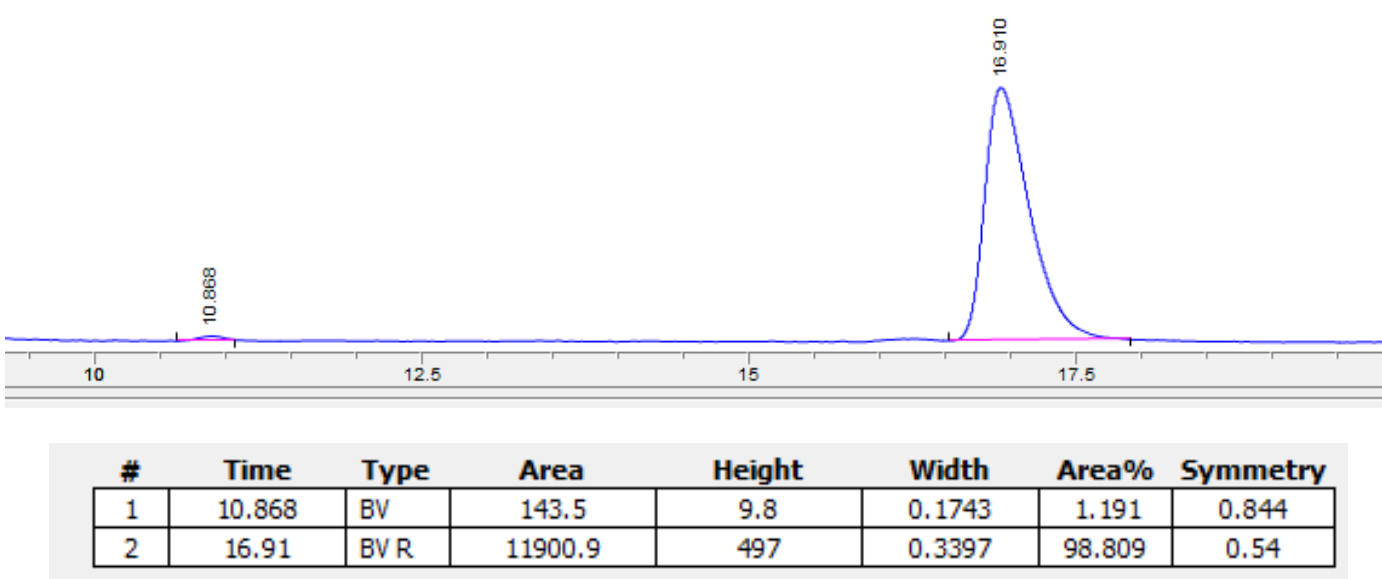


HPLC data for product 23a (see procedure):
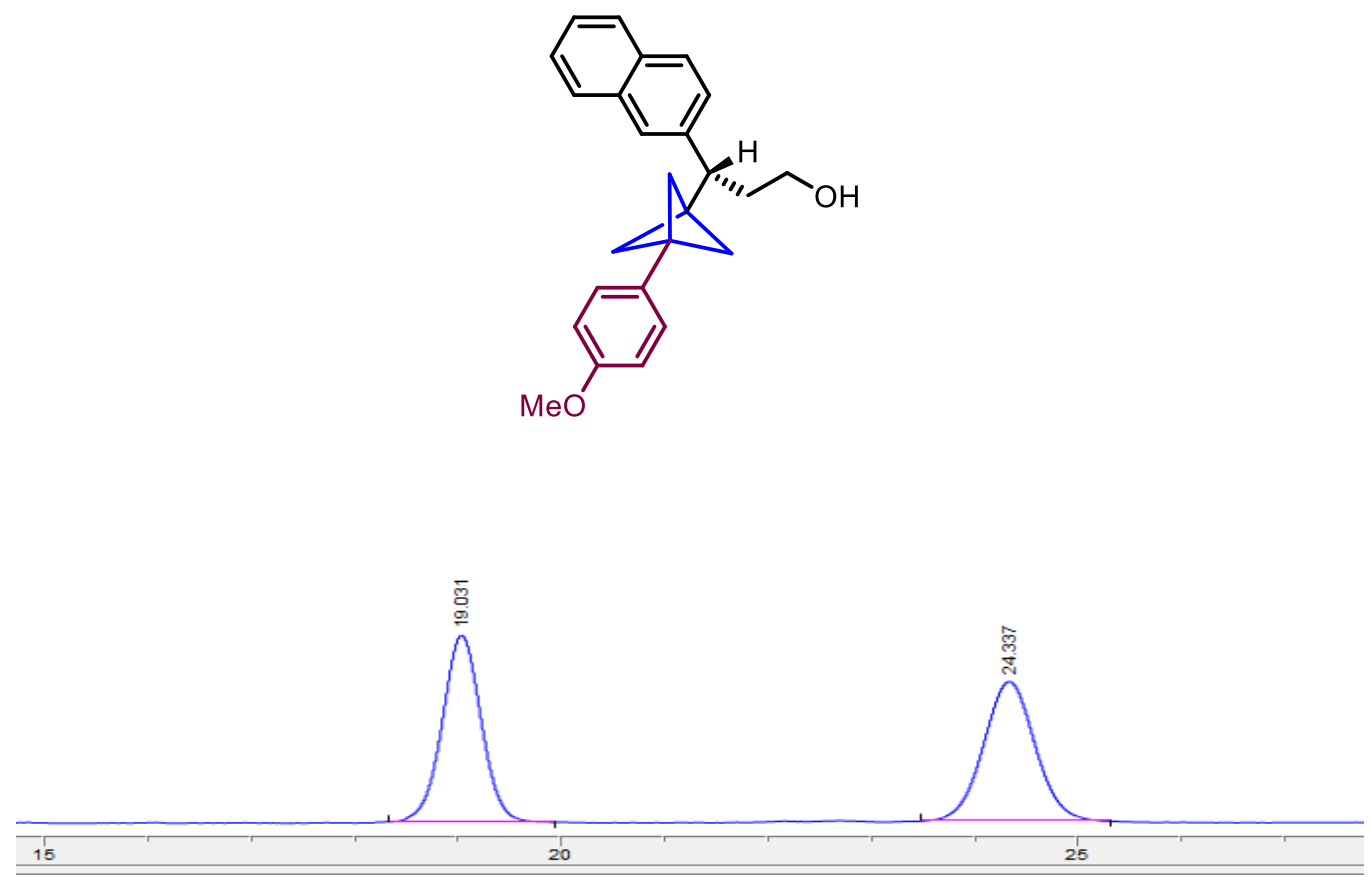

\begin{tabular}{|c|c|c|c|c|c|c|c|}
\hline$\#$ & Time & Type & Area & Height & Width & Area $\%$ & Symmetry \\
\hline 1 & 19.031 & VVR & 11592.8 & 442.1 & 0.3139 & 50.080 & \begin{tabular}{|l|}
0.941 \\
\end{tabular} \\
\hline 2 & 24.337 & VVR & 11556 & 330.5 & 0.4114 & 49.920 & 0.998 \\
\hline
\end{tabular}

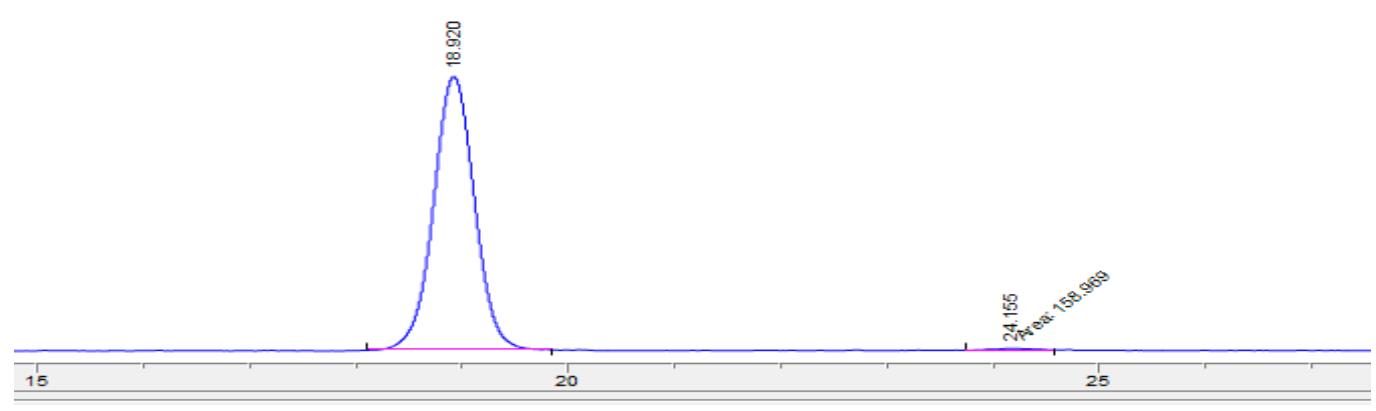

\begin{tabular}{|c|c|l|c|c|c|c|c|}
\multicolumn{1}{l}{ \# } & Time & \multicolumn{1}{c}{ Type } & \multicolumn{1}{c}{ Area } & Height & \multicolumn{1}{c}{ Width } & \multicolumn{1}{c}{ Area\% $\%$ Symmetry } \\
\hline 1 & 18.92 & VV R & 18064.4 & 670.6 & 0.3347 & 99.128 & 1.001 \\
\hline 2 & 24.155 & MM & 159 & 5.2 & 0.5144 & 0.872 & 0.759 \\
\hline
\end{tabular}


HPLC data for product $\mathbf{2 4}$ (see procedure):
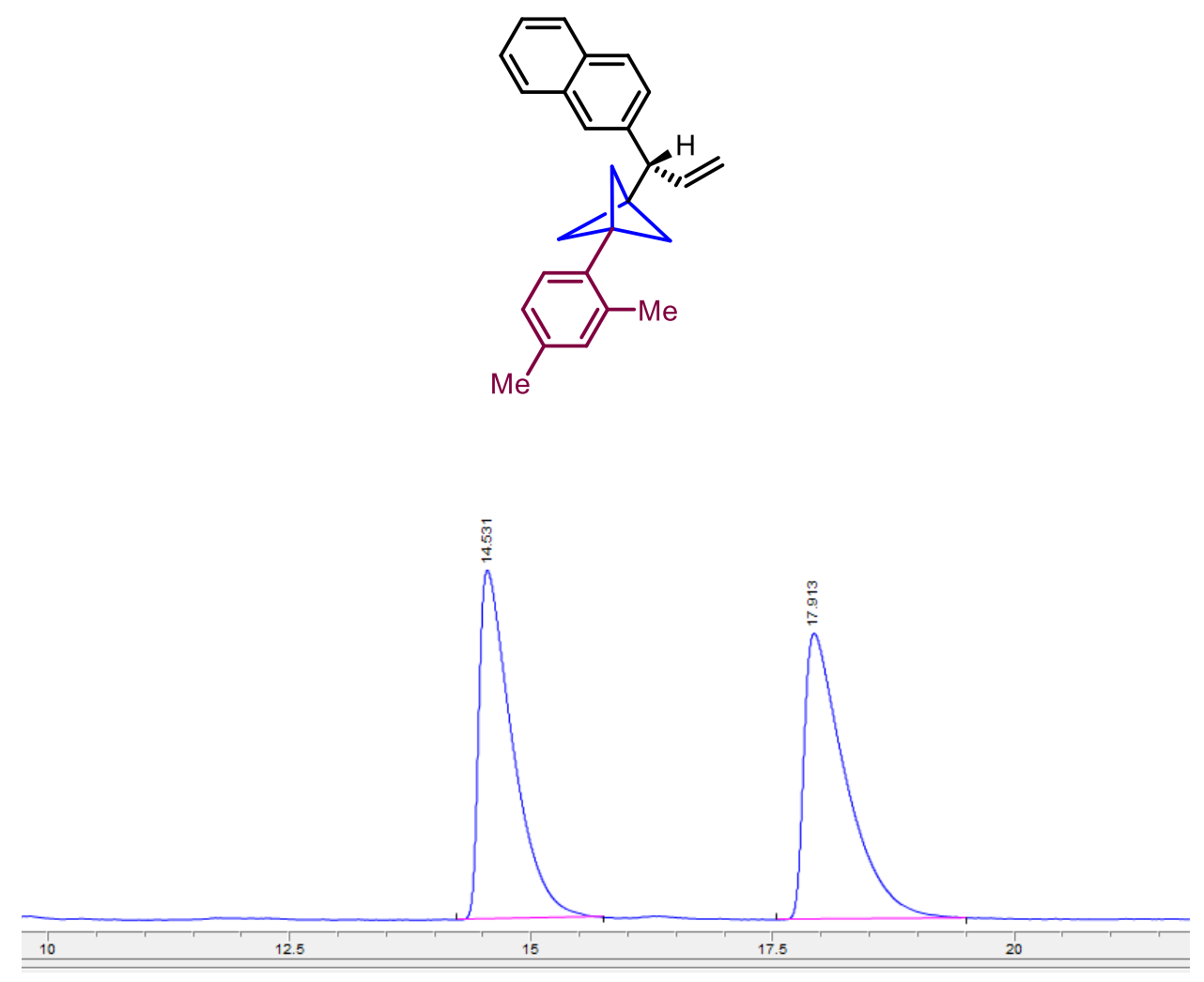

\begin{tabular}{|c|c|c|c|c|c|c|c|}
\hline$\#$ & Time & Type & Area & Height & Width & Area \% & Symmetry \\
\hline 1 & 14.531 & VVR & 22918.9 & 947.2 & 0.3378 & 49.623 & 0.305 \\
\hline 2 & 17.913 & VVR & 23267.5 & 776.4 & 0.4056 & 50.377 & 0.294 \\
\hline
\end{tabular}

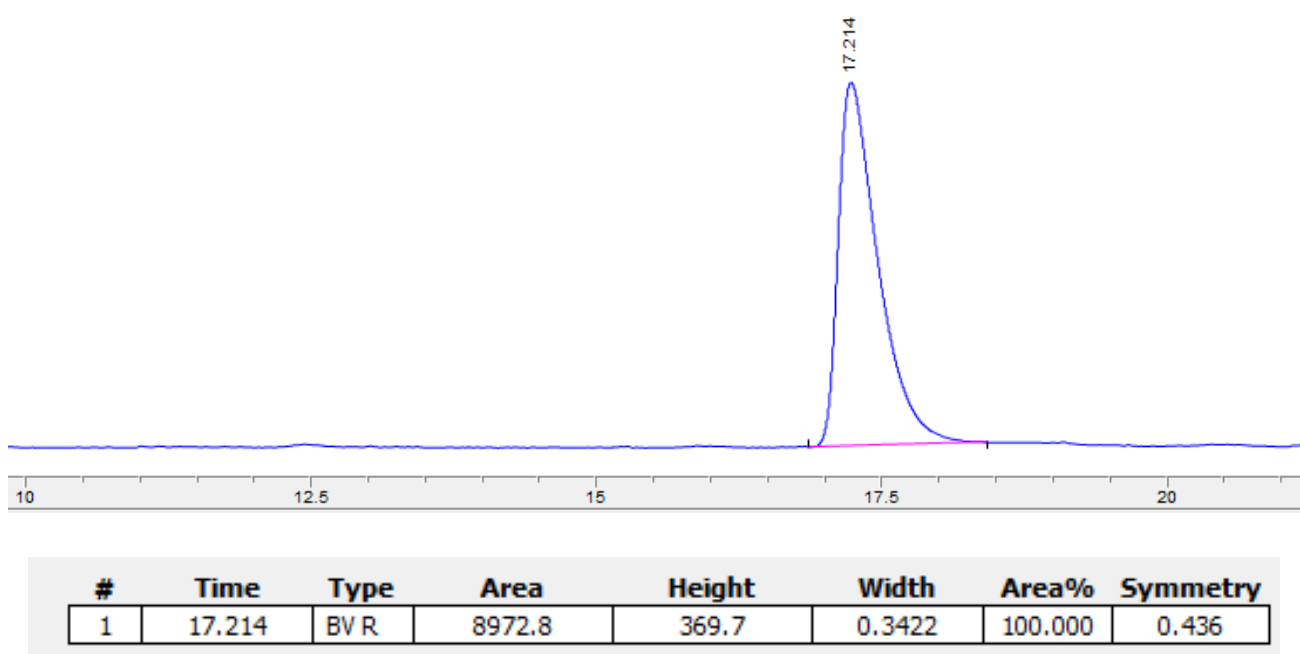


HPLC data for product 25 (see procedure):
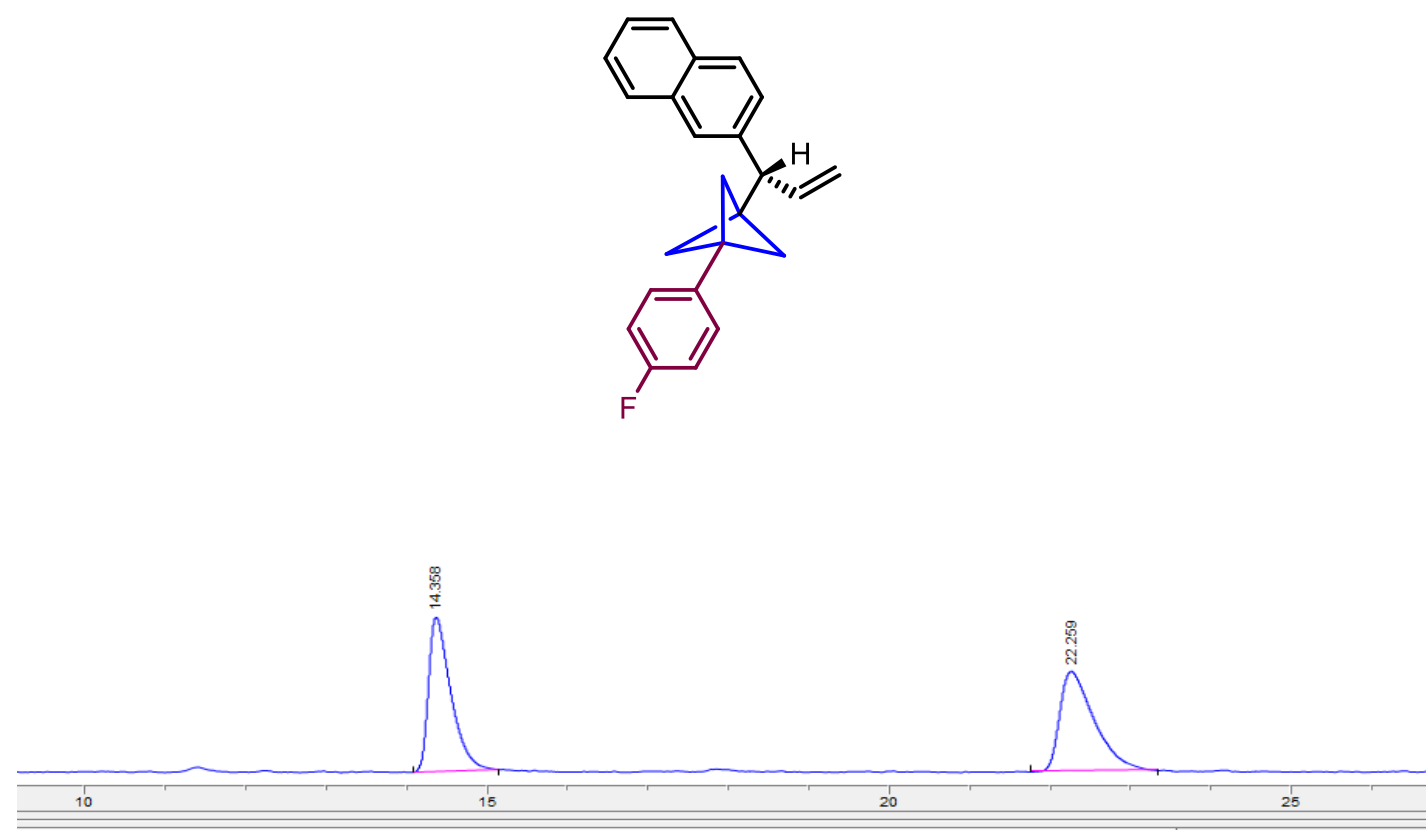

\begin{tabular}{|c|c|c|c|c|c|c|c|}
\hline$\#$ & Time & Type & Area & Height & Width & Area $\%$ & Symmetry \\
\hline 1 & 14.358 & $\mathrm{BB}$ & 4302.5 & 222.5 & 0.2716 & 49.826 & 0.482 \\
\hline 2 & 22.259 & VVR & 4332.6 & 144.8 & 0.3906 & 50.174 & 0.476 \\
\hline
\end{tabular}

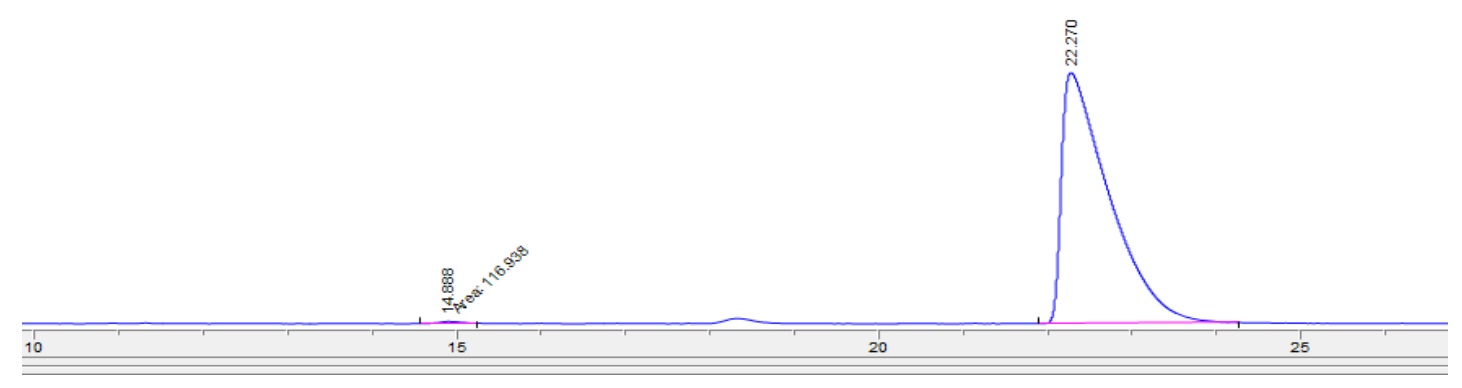

\begin{tabular}{|c|c|l|c|c|c|c|c|} 
\# & \multicolumn{1}{c}{ Time } & \multicolumn{1}{c}{ Type } & Area & Height & \multicolumn{1}{c}{ Width } & \multicolumn{2}{c}{ Area\% $\%$ Symmetry } \\
\hline 1 & 14.888 & MM & 116.9 & 6.5 & 0.299 & 0.407 & 0.802 \\
\hline 2 & 22.27 & BVR & 28636.9 & 730.2 & 0.5099 & 99.593 & 0.231 \\
\hline
\end{tabular}


HPLC data for product 26 (see procedure):
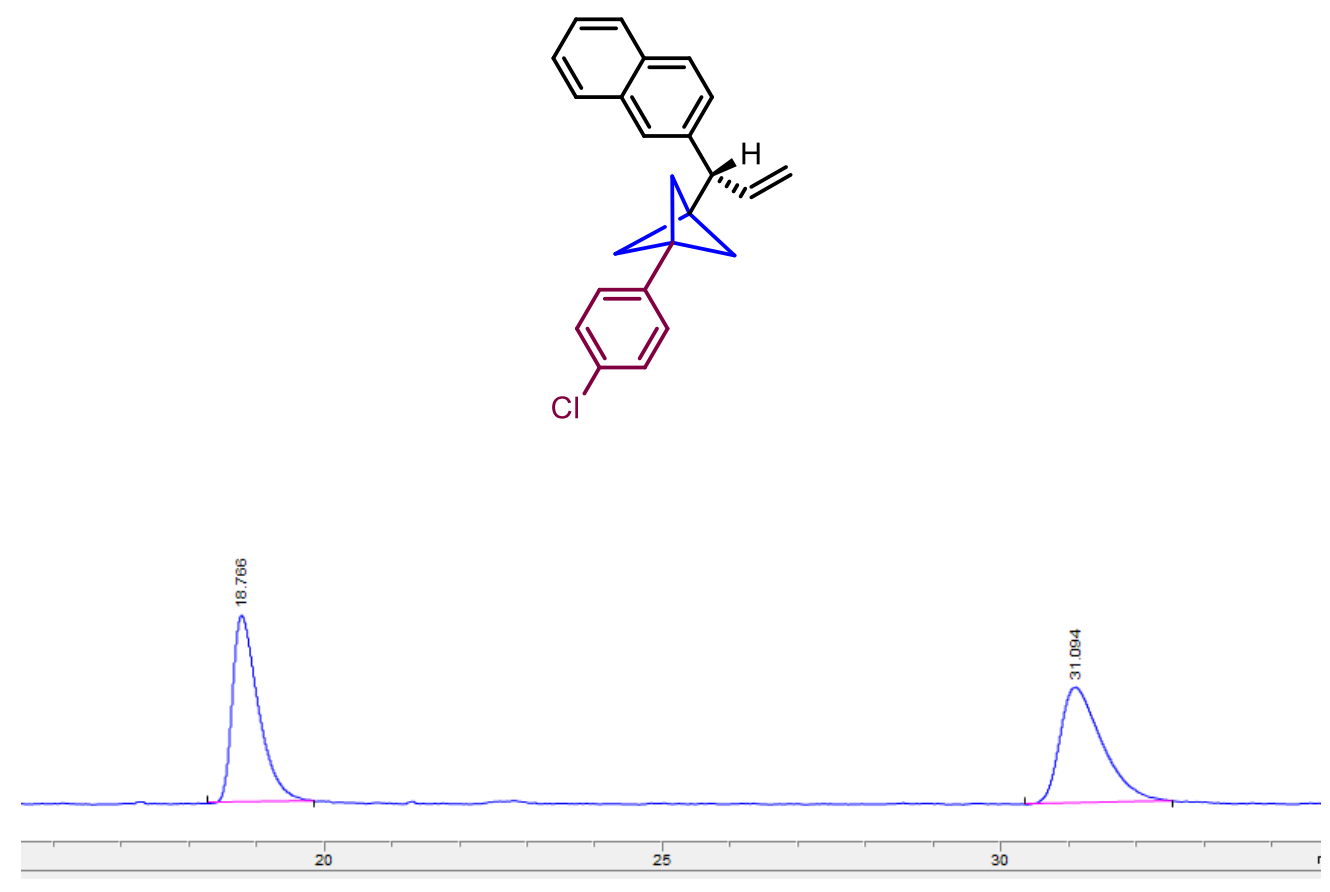

\begin{tabular}{|c|c|c|c|c|c|c|c|}
\hline$\#$ & Time & Type & Area & Height & Width & Area $\%$ & Symmetry \\
\hline 1 & 18.766 & VVR & 3883.8 & 143.8 & 0.3767 & 49.893 & 0.514 \\
\hline 2 & 31.094 & VVR & 3900.5 & 89.3 & 0.5176 & 50.107 & 0.583 \\
\hline
\end{tabular}

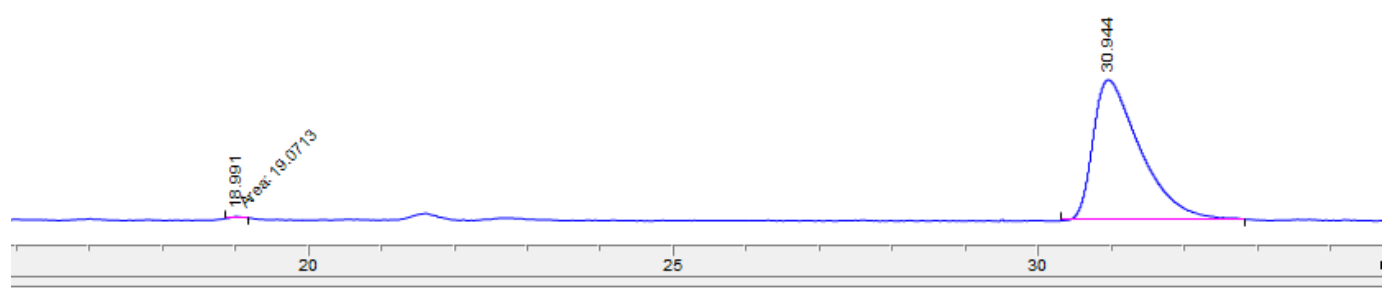

\begin{tabular}{|c|c|c|c|c|c|c|c|}
\hline$\#$ & Time & Type & Area & Height & Width & Area $\%$ & Symmetry \\
\hline 1 & 18.991 & MM & 19.1 & 1.8 & 0.1791 & 0.289 & 0.735 \\
\hline 2 & 30.944 & VVR & 6585.3 & 146.2 & 0.5435 & 99.711 & 0.451 \\
\hline
\end{tabular}


HPLC data for product 27 (see procedure):
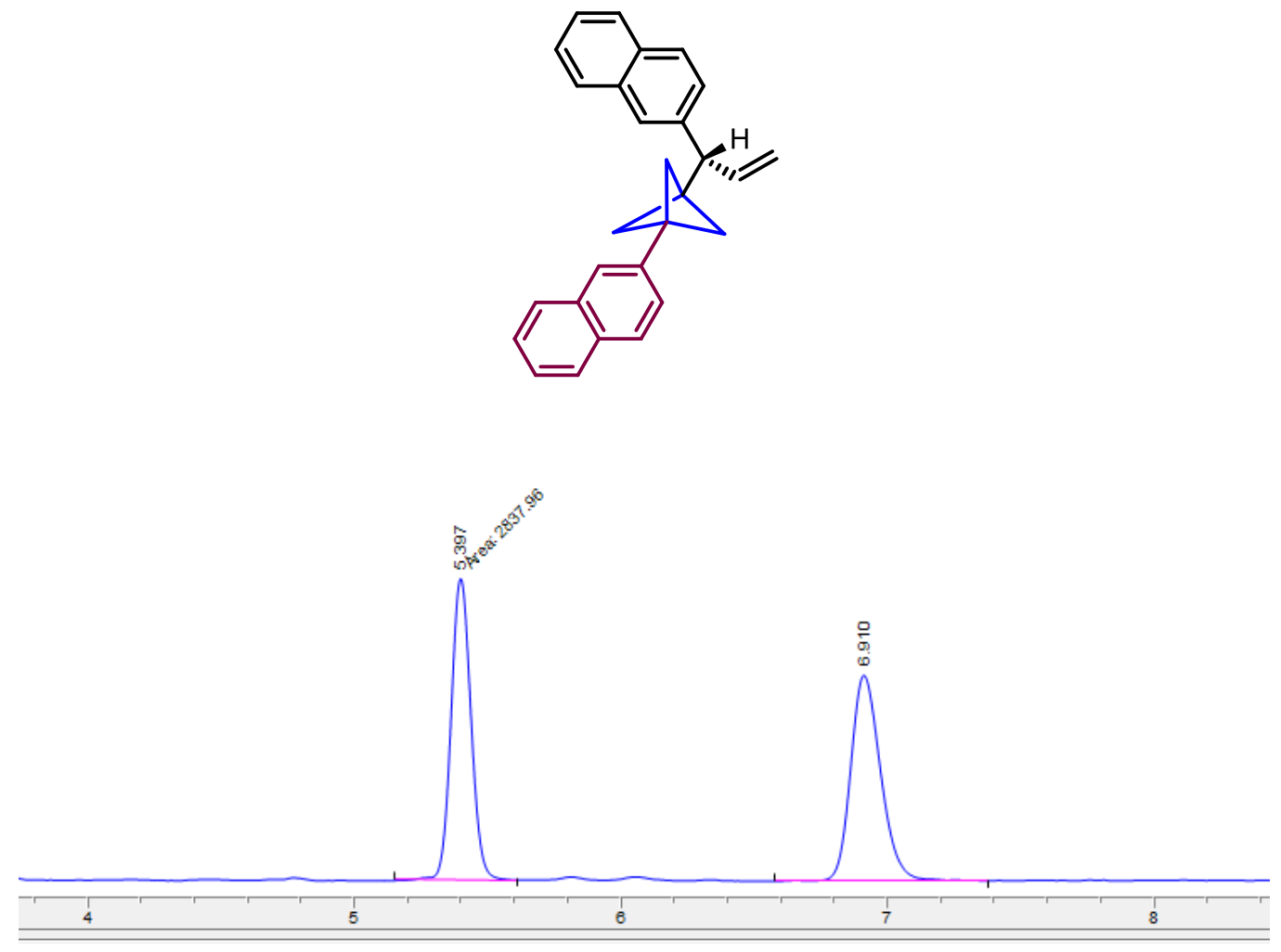

\begin{tabular}{|c|c|c|c|c|c|c|c|}
\hline$\#$ & Time & Type & Area & Height & Width & Area $\%$ & Symmetry \\
\hline 1 & 5.397 & MM & 2838 & 538.1 & 0.0879 & 49.734 & 0.904 \\
\hline 2 & 6.91 & VVR & 2868.3 & 365.4 & 0.1208 & 50.266 & 0.759 \\
\hline
\end{tabular}

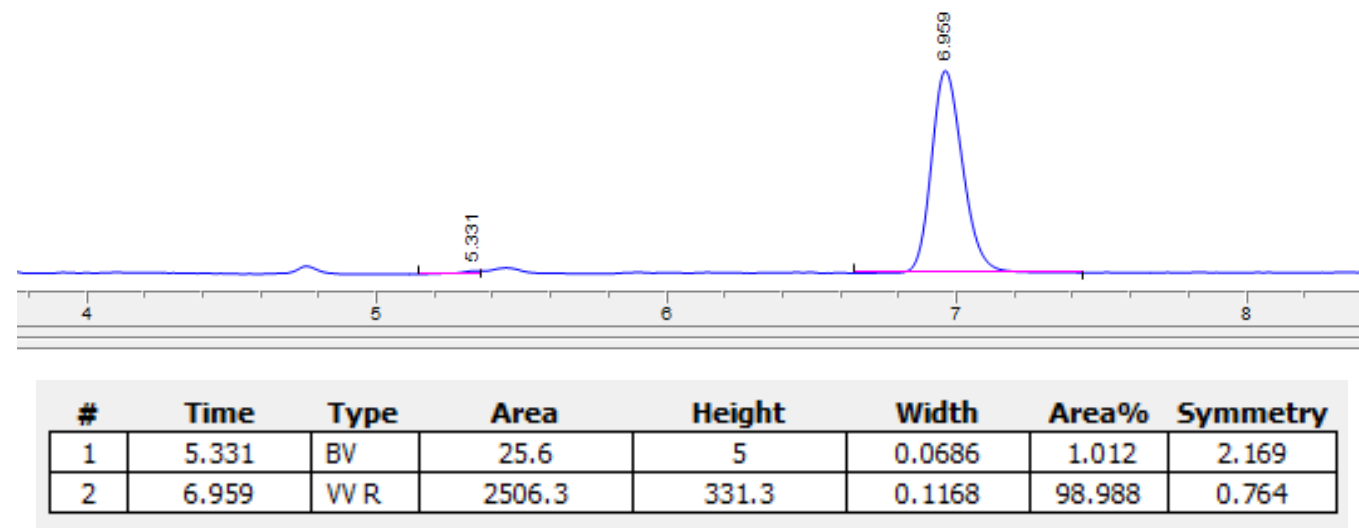


HPLC data for product $\mathbf{2 8}$ (see procedure):
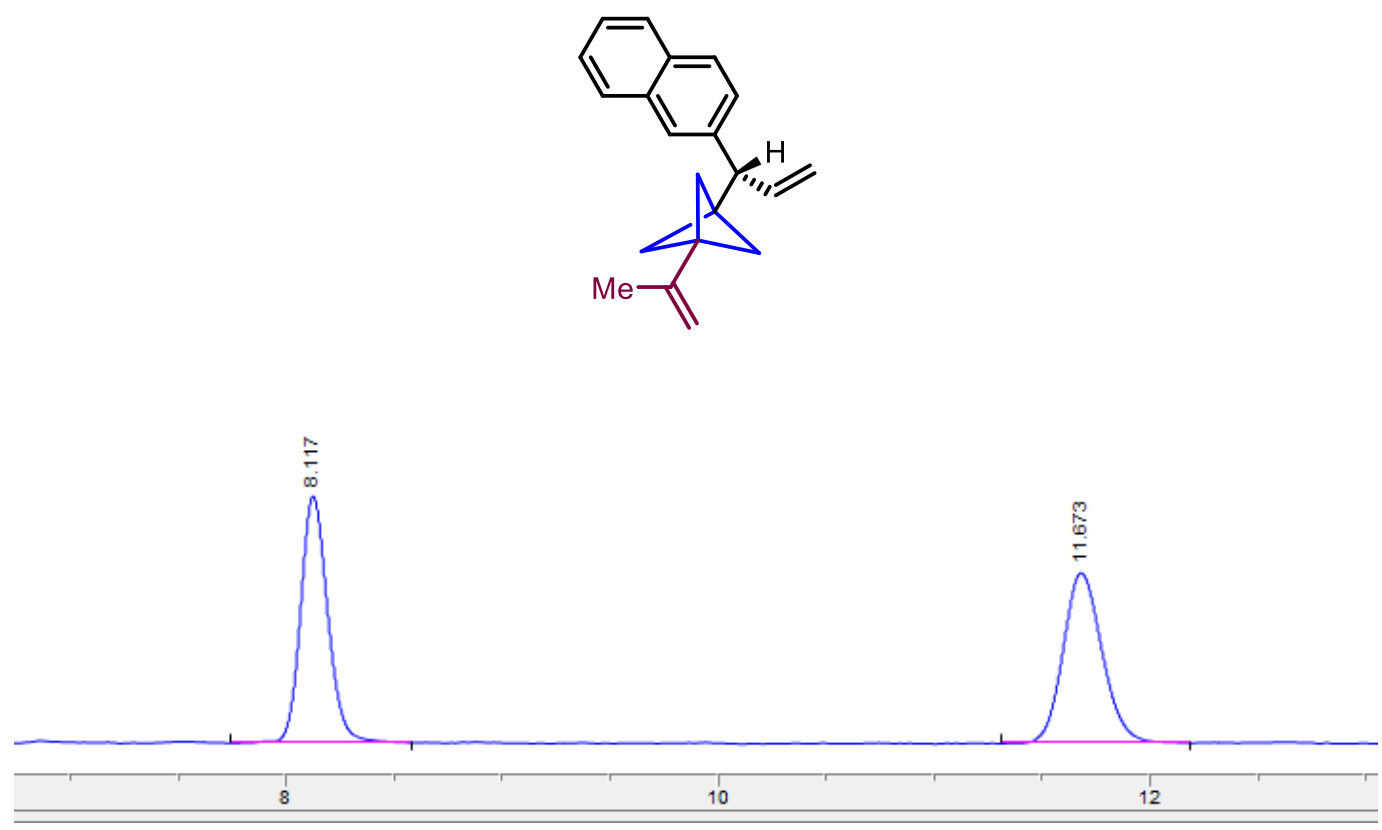

\begin{tabular}{|c|c|c|c|c|c|c|c|}
\hline$\#$ & Time & Type & Area & Height & Width & Area $\%$ & Symmetry \\
\hline 1 & 8.117 & VBR & 2071.5 & 235.8 & 0.1345 & 50.166 & \begin{tabular}{|l}
0.817 \\
\end{tabular} \\
\hline 2 & 11.673 & VVR & 2057.8 & 162.7 & 0.1946 & 49.834 & 0.832 \\
\hline
\end{tabular}

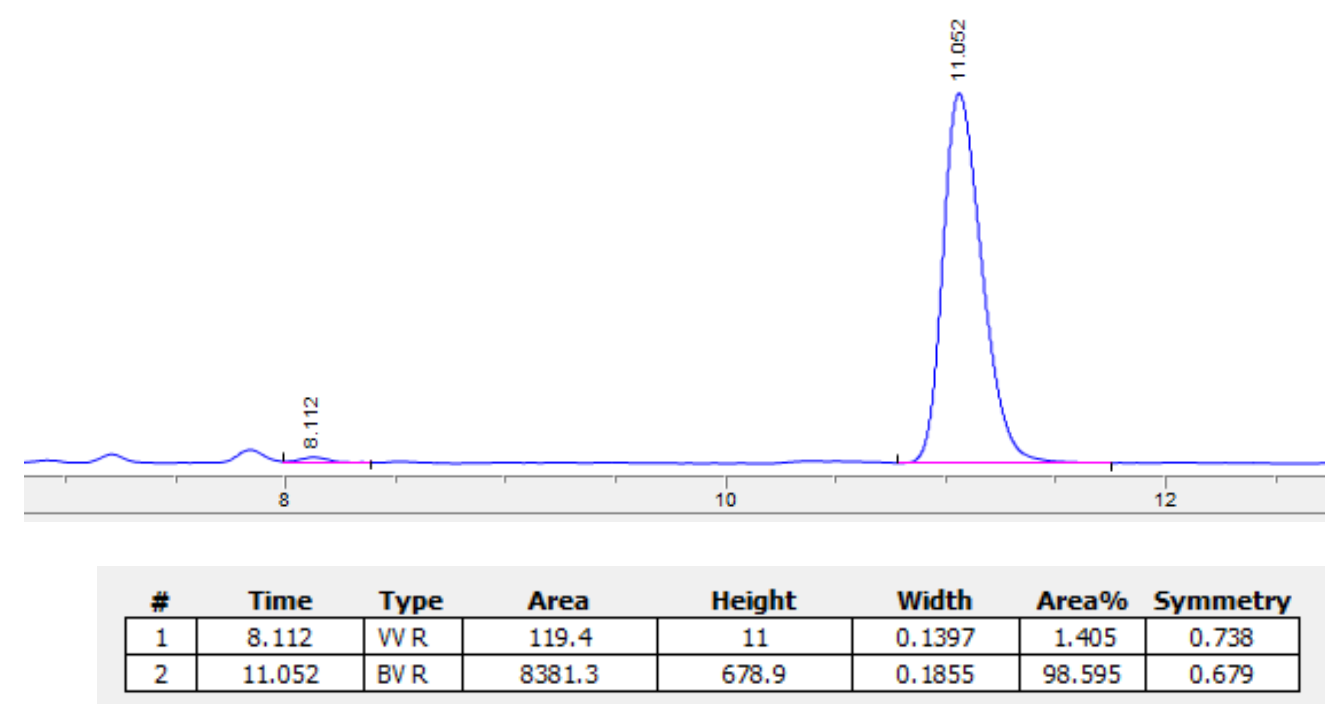


HPLC data for product 29 (see procedure):
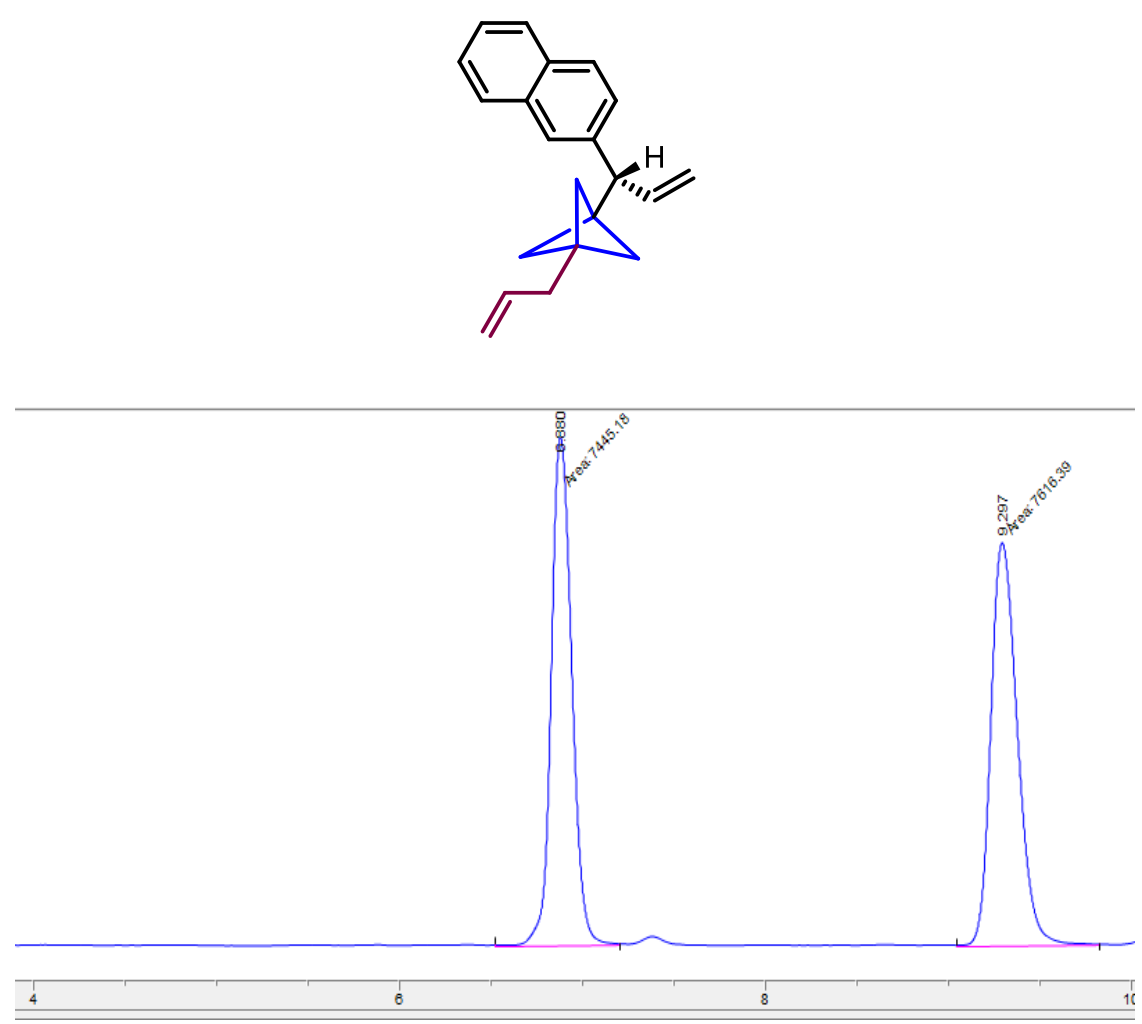

\begin{tabular}{|c|c|l|c|c|c|c|c|} 
\# & Time & \multicolumn{1}{c}{ Type } & Area & Height & \multicolumn{1}{c}{ Width } & \multicolumn{2}{c}{ Area\% } \\
\hline 1 & 6.88 & MM & 7445.2 & 946.3 & 0.1311 & 49.432 & 0.796 \\
\hline 2 & 9.297 & MM & 7616.4 & 750.8 & 0.1691 & 50.568 & 0.722 \\
\hline
\end{tabular}

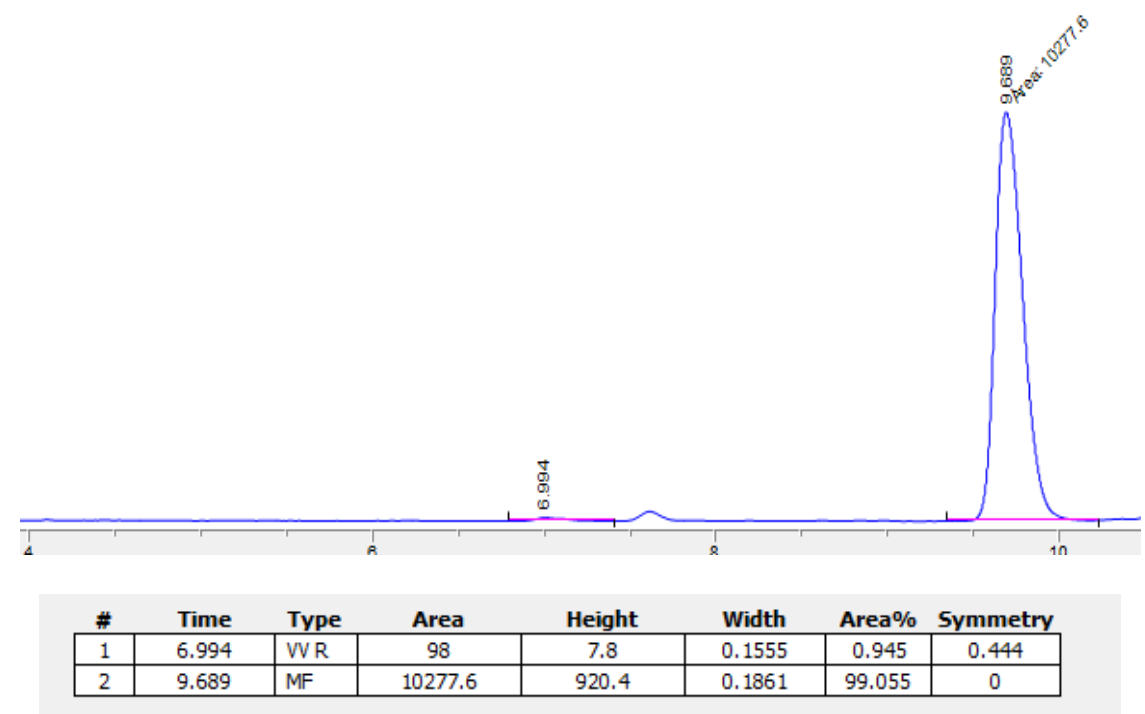


HPLC data for product 30a (see procedure):<smiles>CCC12CCC([C@H](CCO)c3ccc4ccccc4c3)(C1)C2</smiles>

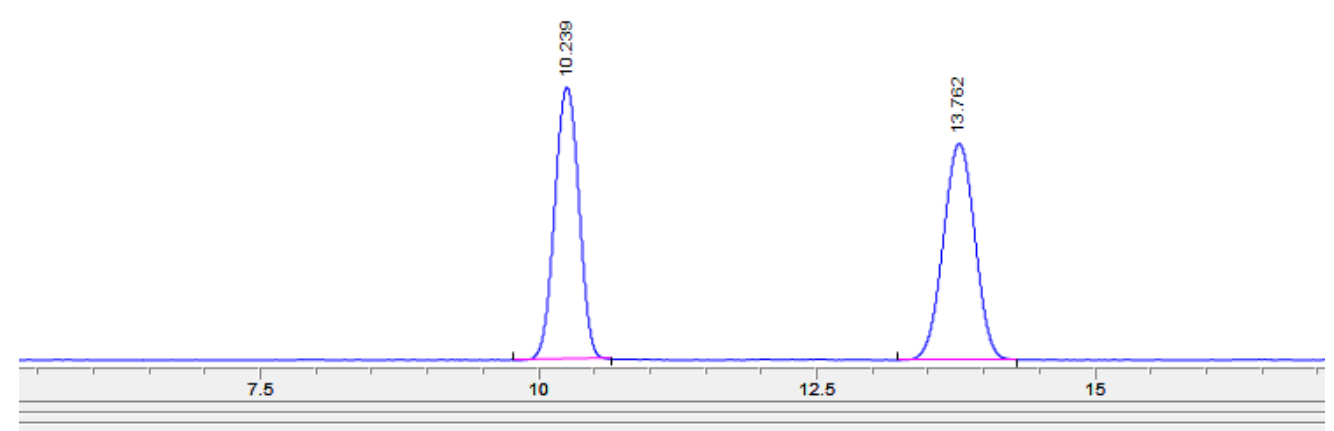

\begin{tabular}{|c|c|l|c|c|c|c|c|}
\multicolumn{1}{c}{ \# } & Time & \multicolumn{1}{c}{ Type } & \multicolumn{1}{c}{ Area } & \multicolumn{1}{c}{ Height } & Width & \multicolumn{2}{c|}{ Area\% $\%$ Symmetry } \\
\hline 1 & 10.239 & VB R & 10446.6 & 686.5 & 0.238 & 49.206 & 0.986 \\
\hline 2 & 13.762 & VVR & 10783.9 & 545.4 & 0.2872 & 50.794 & 0.985 \\
\hline
\end{tabular}

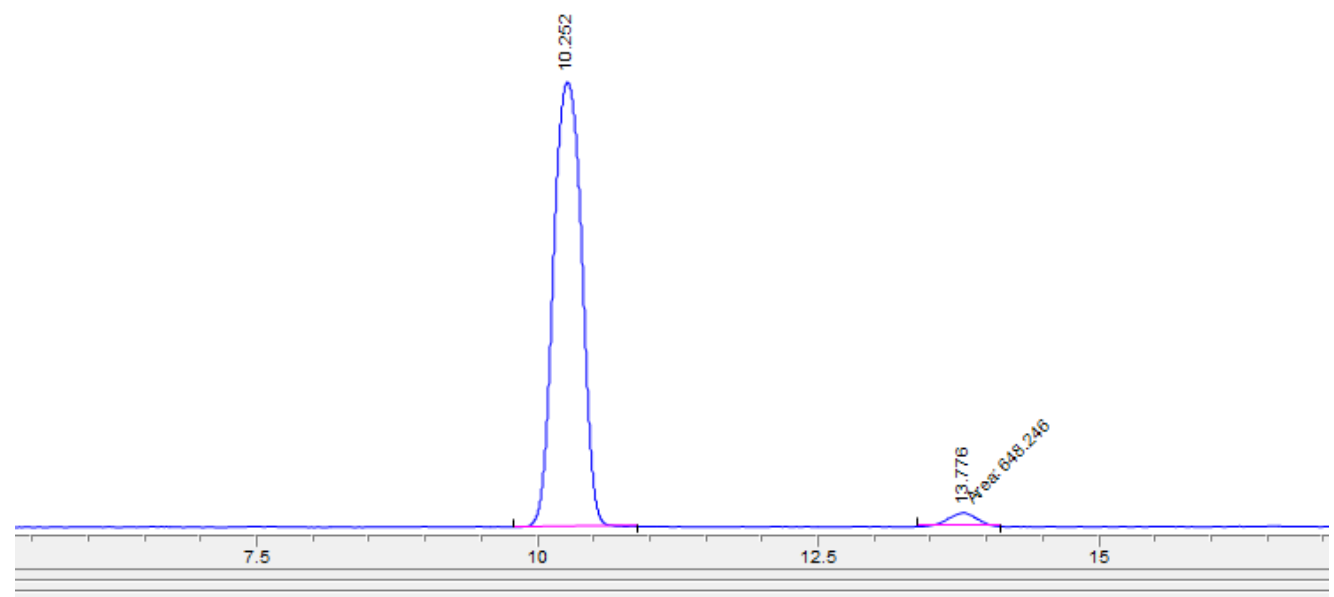

\begin{tabular}{|c|c|c|c|c|c|c|c|}
\hline$\#$ & Time & Type & Area & Height & Width & Area\% & Symmetry \\
\hline 1 & 10.252 & VVR & 19430.7 & 1109 & 0.2618 & 96.772 & 0.95 \\
\hline 2 & 13.776 & MM & 648.2 & 35.1 & 0.3082 & 3.228 & 1.162 \\
\hline
\end{tabular}


HPLC data for product 31a (see procedure):
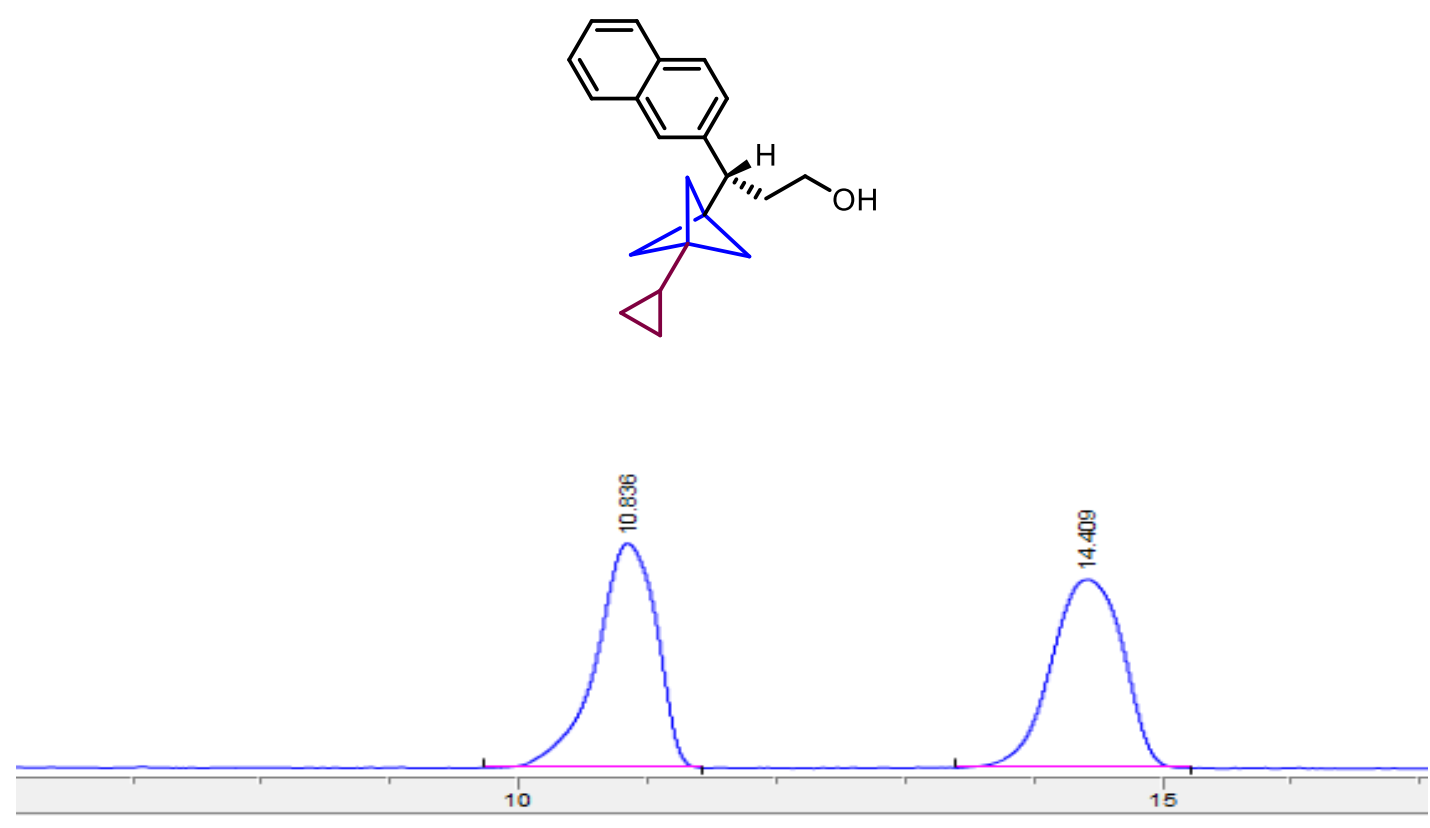

\begin{tabular}{|c|c|l|c|c|c|c|c|} 
\# & Time & \multicolumn{1}{c}{ Type } & \multicolumn{1}{c}{ Area } & Height & \multicolumn{1}{c}{ Width } & \multicolumn{2}{c}{ Area\% } \\
\hline 1 & 10.836 & VV R & 12956.4 & 396.5 & 0.3878 & 49.960 & 1.118 \\
\hline 2 & 14.409 & VVR & 12977 & 333.3 & 0.4567 & 50.040 & 1.018 \\
\hline
\end{tabular}

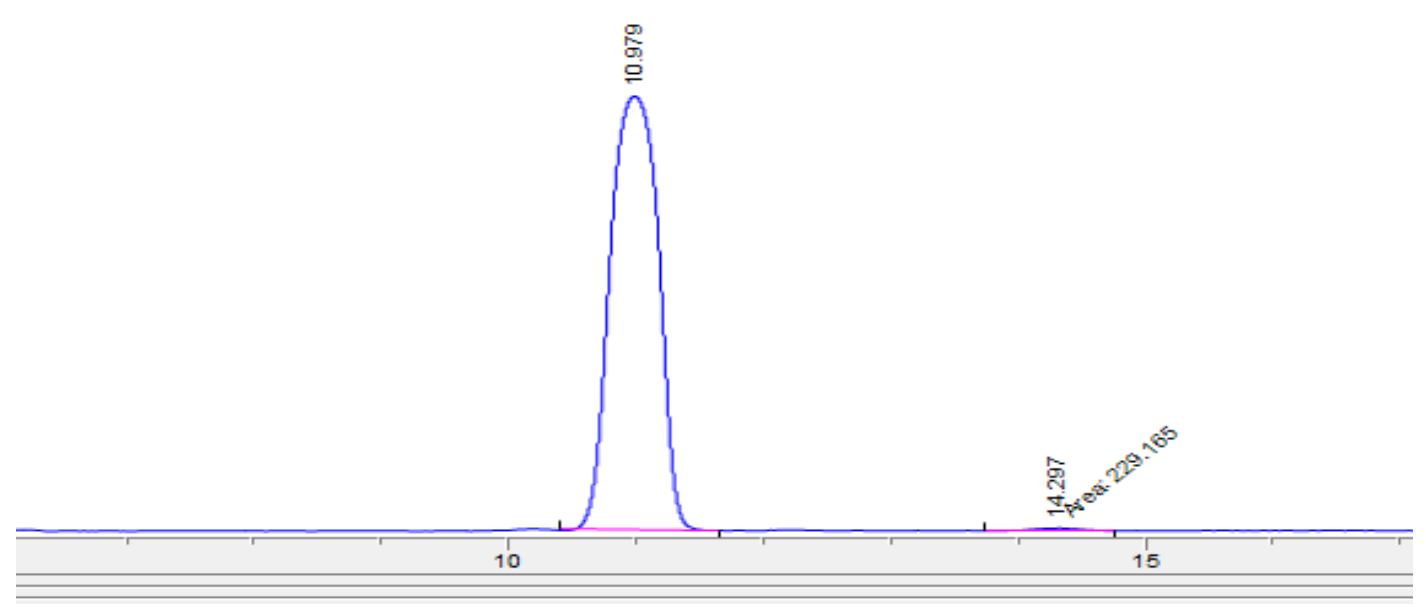

\begin{tabular}{|c|c|l|c|c|c|c|c|}
\multicolumn{1}{l}{ \# } & \multicolumn{1}{c}{ Time } & \multicolumn{1}{c}{ Type } & \multicolumn{1}{c}{ Area } & Height & \multicolumn{1}{c}{ Width } & \multicolumn{2}{c}{ Area\% $\%$ Symmetry } \\
\hline 1 & 10.979 & VV R & 32859.1 & 1248.7 & 0.308 & 99.307 & 1.002 \\
\hline 2 & 14.297 & MM & 229.2 & 8.4 & 0.4524 & 0.693 & 1.191 \\
\hline
\end{tabular}


HPLC data for product 32a (see procedure):
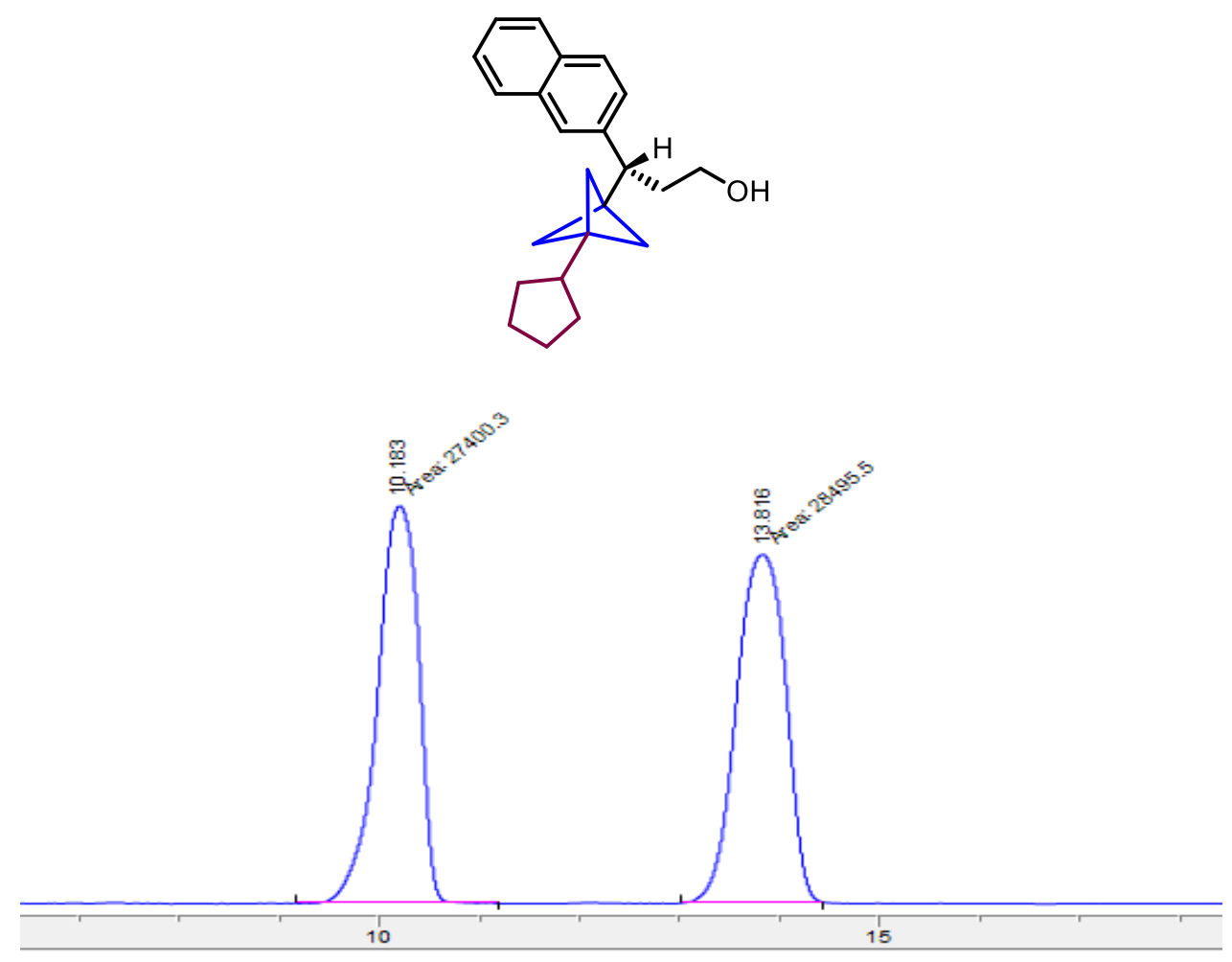

\begin{tabular}{|c|c|l|c|c|c|c|c|} 
\# & \multicolumn{1}{c}{ Time } & \multicolumn{1}{c}{ Type } & \multicolumn{1}{c}{ Area } & Height & Width & \multicolumn{2}{c|}{ Area\% } \\
\hline 1 & 10.183 & MM & 27400.3 & 953.6 & 0.4789 & 49.020 & 1.119 \\
\hline 2 & 13.816 & MM & 28495.5 & 834.9 & 0.5689 & 50.980 & 1.119 \\
\hline
\end{tabular}

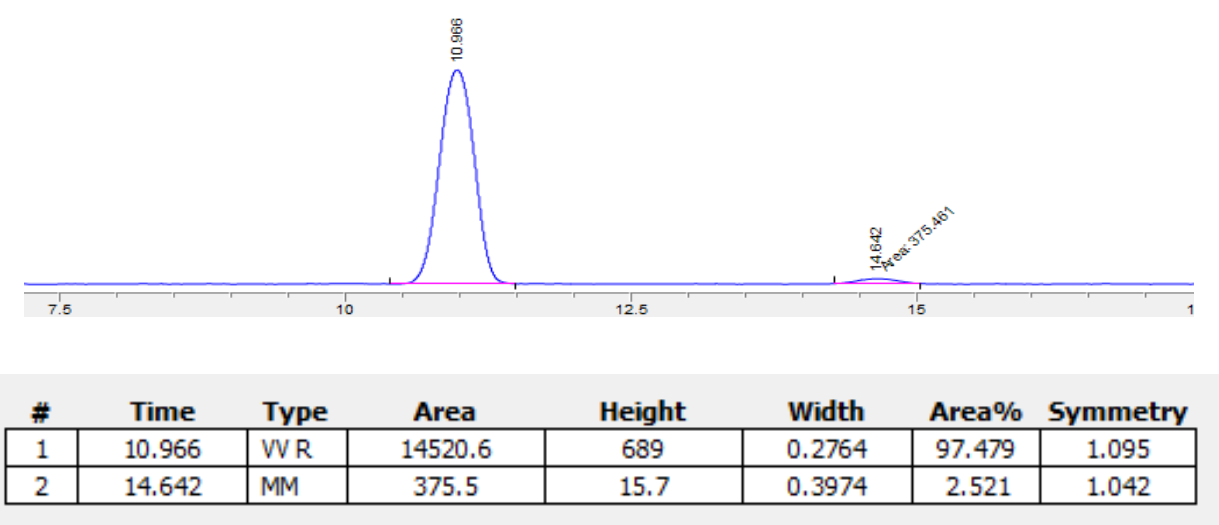


HPLC data for product $\mathbf{3 4 a}$ (see procedure):
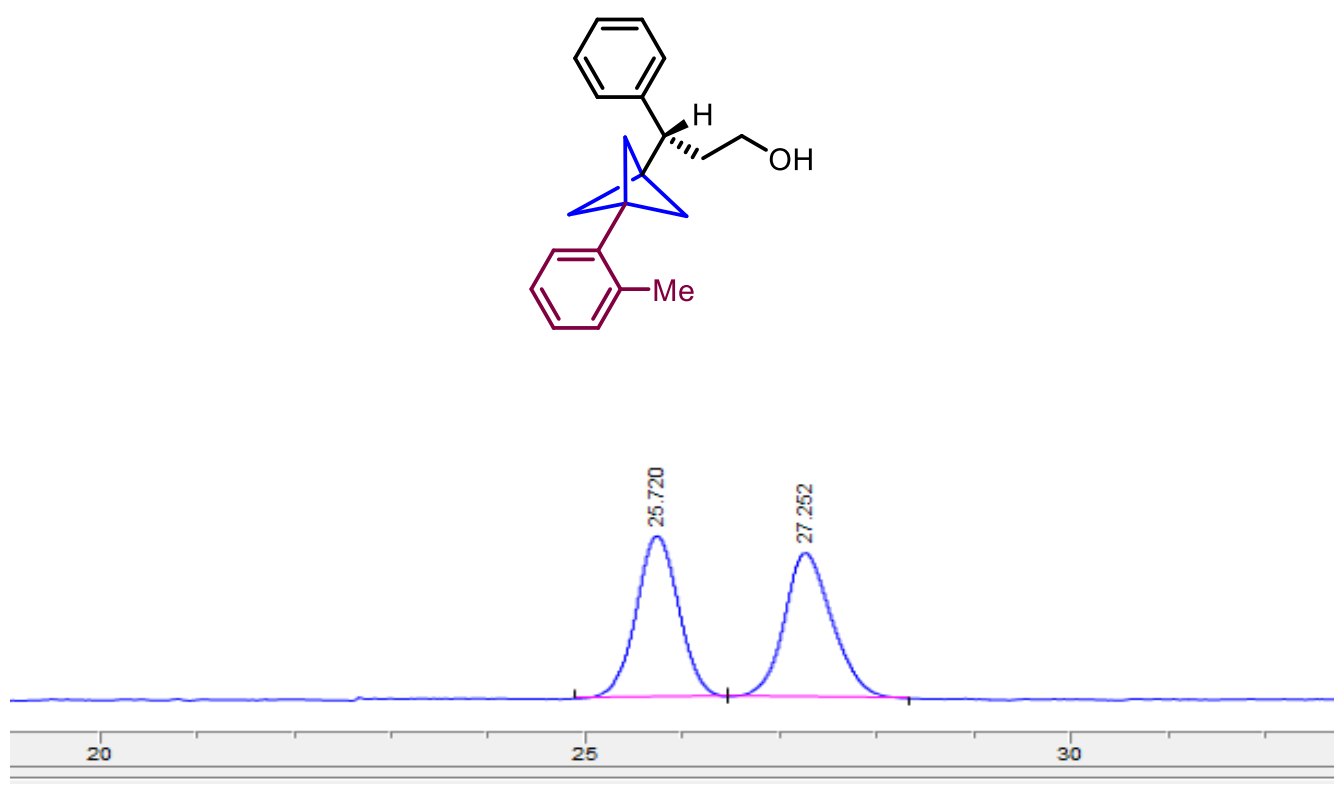

\begin{tabular}{|c|c|l|c|c|c|c|c|}
\multicolumn{1}{l}{ \# } & \multicolumn{1}{c}{ Time } & \multicolumn{1}{c}{ Type } & \multicolumn{1}{c}{ Area } & Height & \multicolumn{1}{c}{ Width } & \multicolumn{2}{c|}{ Area\% $\%$ Symmetry } \\
\hline 1 & 25.72 & VV R & 4642.6 & 151.6 & 0.4277 & 49.930 & 0.943 \\
\hline 2 & 27.252 & BB & 4655.6 & 135.5 & 0.4495 & 50.070 & 0.775 \\
\hline
\end{tabular}

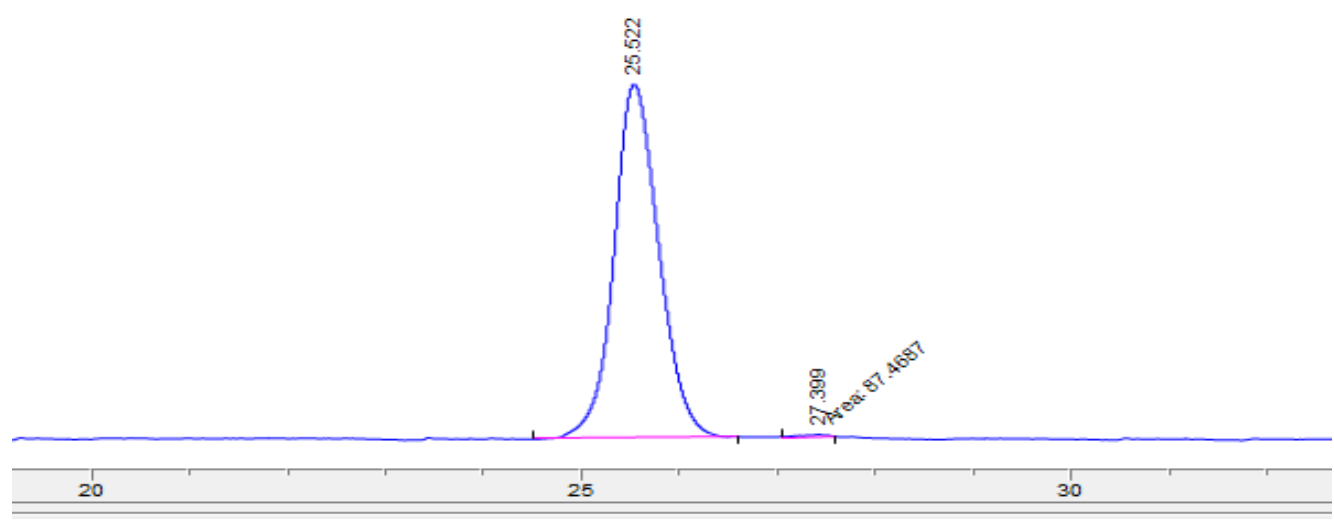

\begin{tabular}{|c|c|l|c|c|c|c|c|} 
\# & \multicolumn{1}{c}{ Time } & \multicolumn{1}{c}{ Type } & \multicolumn{1}{c}{ Area } & Height & \multicolumn{1}{c}{ Width } & \multicolumn{2}{c|}{ Area\% $\%$ Symmetry } \\
\hline 1 & 25.522 & VV R & 11113.5 & 347.6 & 0.455 & 99.219 & 0.809 \\
\hline 2 & 27.399 & MM & 87.5 & 3.8 & 0.3866 & 0.781 & 2.804 \\
\hline
\end{tabular}


HPLC data for product 35 (see procedure):<smiles>O=C1c2ccccc2C(=O)N1C[C@]1(c2ccc(Cl)cc2)CC2(c3ccccc3)CC1C2</smiles>

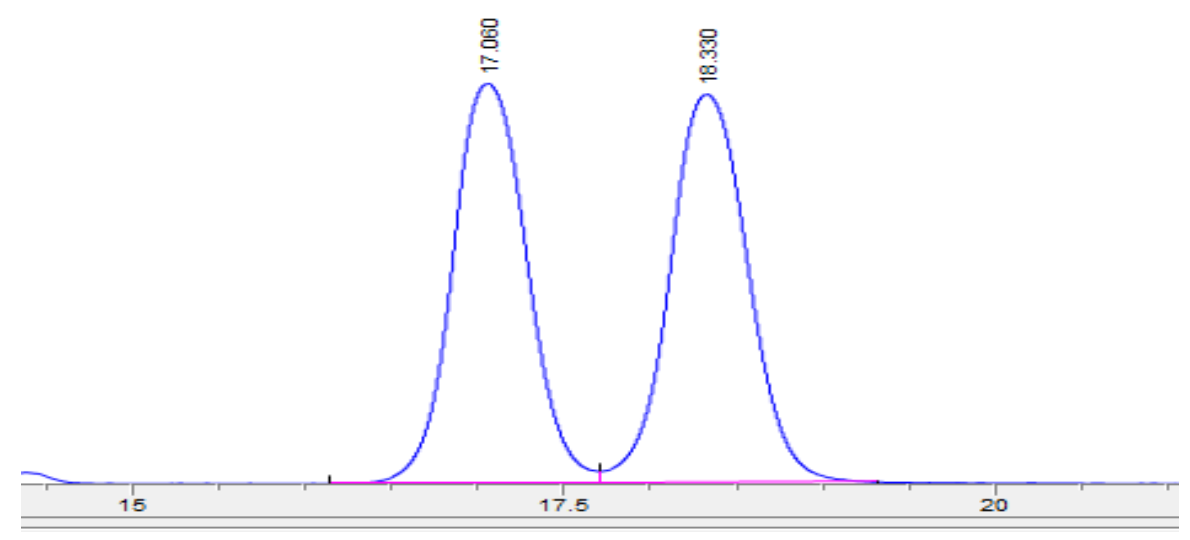

\begin{tabular}{|c|c|l|c|c|c|c|c|}
$\#$ & Time & \multicolumn{1}{c}{ Type } & \multicolumn{1}{c}{ Area } & \multicolumn{1}{c}{ Height } & \multicolumn{1}{c}{ Width } & \multicolumn{1}{c}{ Area\% $\%$ Symmetry } \\
\hline 1 & 17.06 & VV R & 37602 & 1253 & 0.3571 & 49.477 & 0.903 \\
\hline 2 & 18.33 & VB & 38397 & 1217.9 & 0.3738 & 50.523 & 0.892 \\
\hline
\end{tabular}

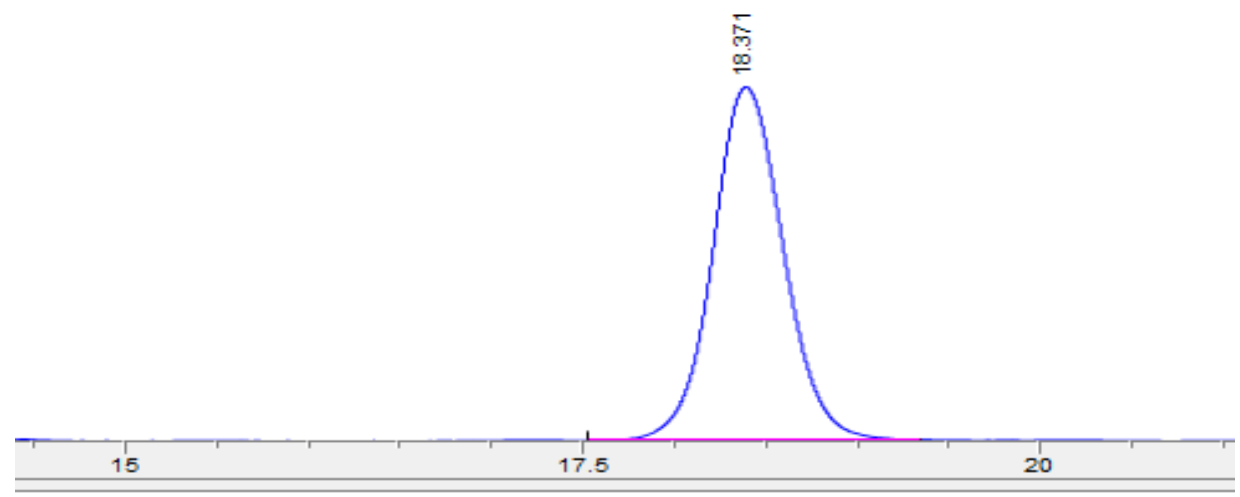

\begin{tabular}{|c|c|l|c|c|c|c|c|}
\multicolumn{1}{c}{ \# } & Time & \multicolumn{1}{c}{ Type } & \multicolumn{1}{c}{ Area } & \multicolumn{1}{c}{ Height } & Width & \multicolumn{1}{c}{ Area\% $\%$ Symmetry } \\
\hline 1 & 18.371 & VVR & 26007.6 & 965.8 & 0.3291 & 100.000 & 0.878 \\
\hline
\end{tabular}


HPLC data for product 39 (see procedure):
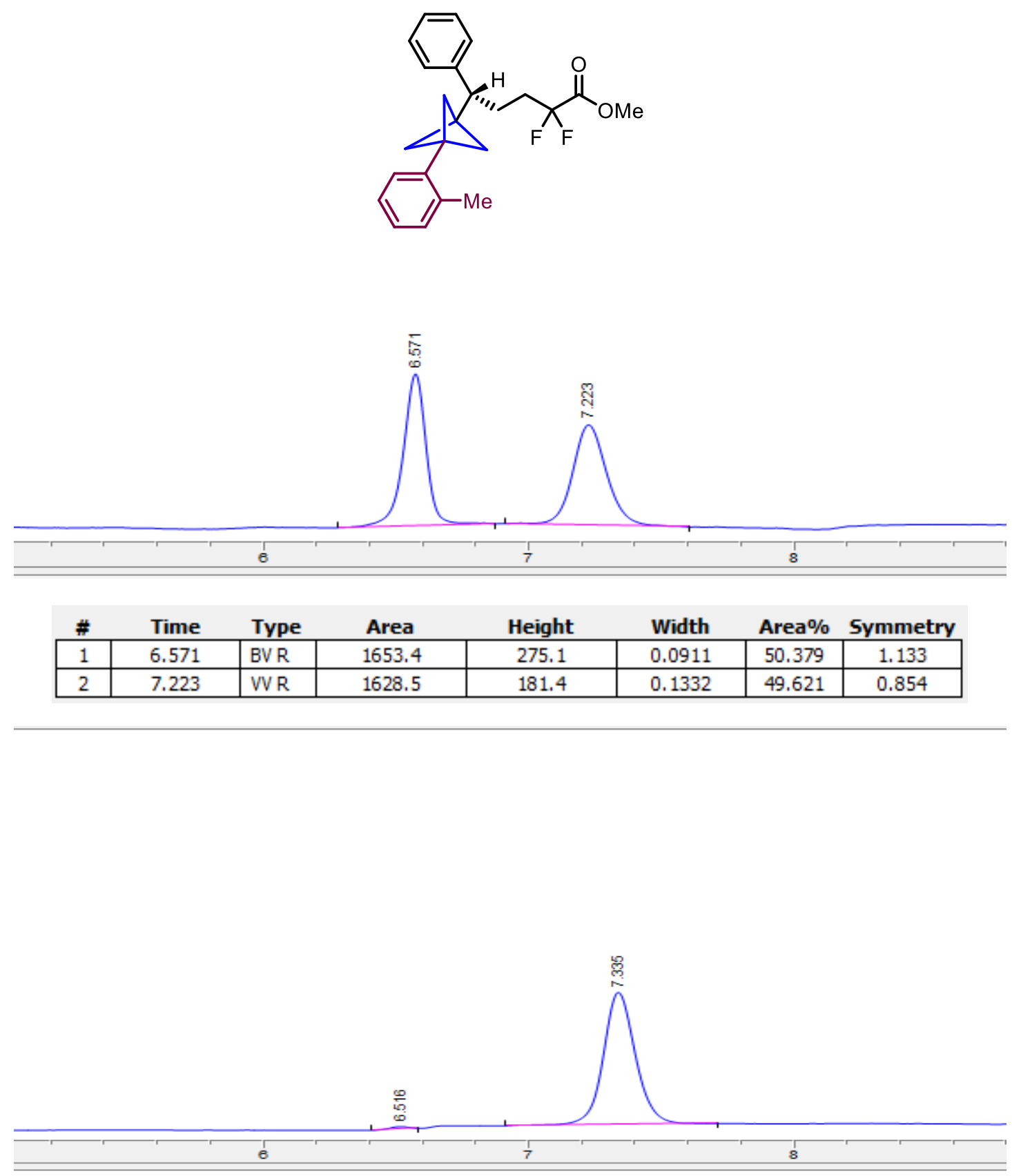

\begin{tabular}{|c|c|c|c|c|c|c|c|}
\hline$\#$ & Time & Type & Area & Height & Width & Area $\%$ & Symmetr \\
\hline 1 & 6.516 & $\mathrm{BB}$ & 46.9 & 9.3 & 0.0622 & 1.109 & 1.525 \\
\hline 2 & 7.335 & VVR & 4186.8 & 493.1 & 0.1281 & 98.891 & 0.869 \\
\hline
\end{tabular}


VI. NMR Spectra

${ }^{1} \mathrm{H}$ NMR (400 MHz, $\mathrm{CDCl}_{3}$ ) spectrum for product 4 (see procedure):

51752 yu534.10.fid

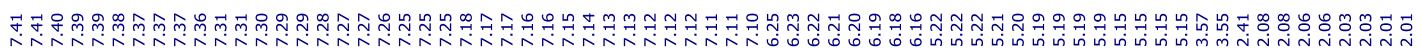
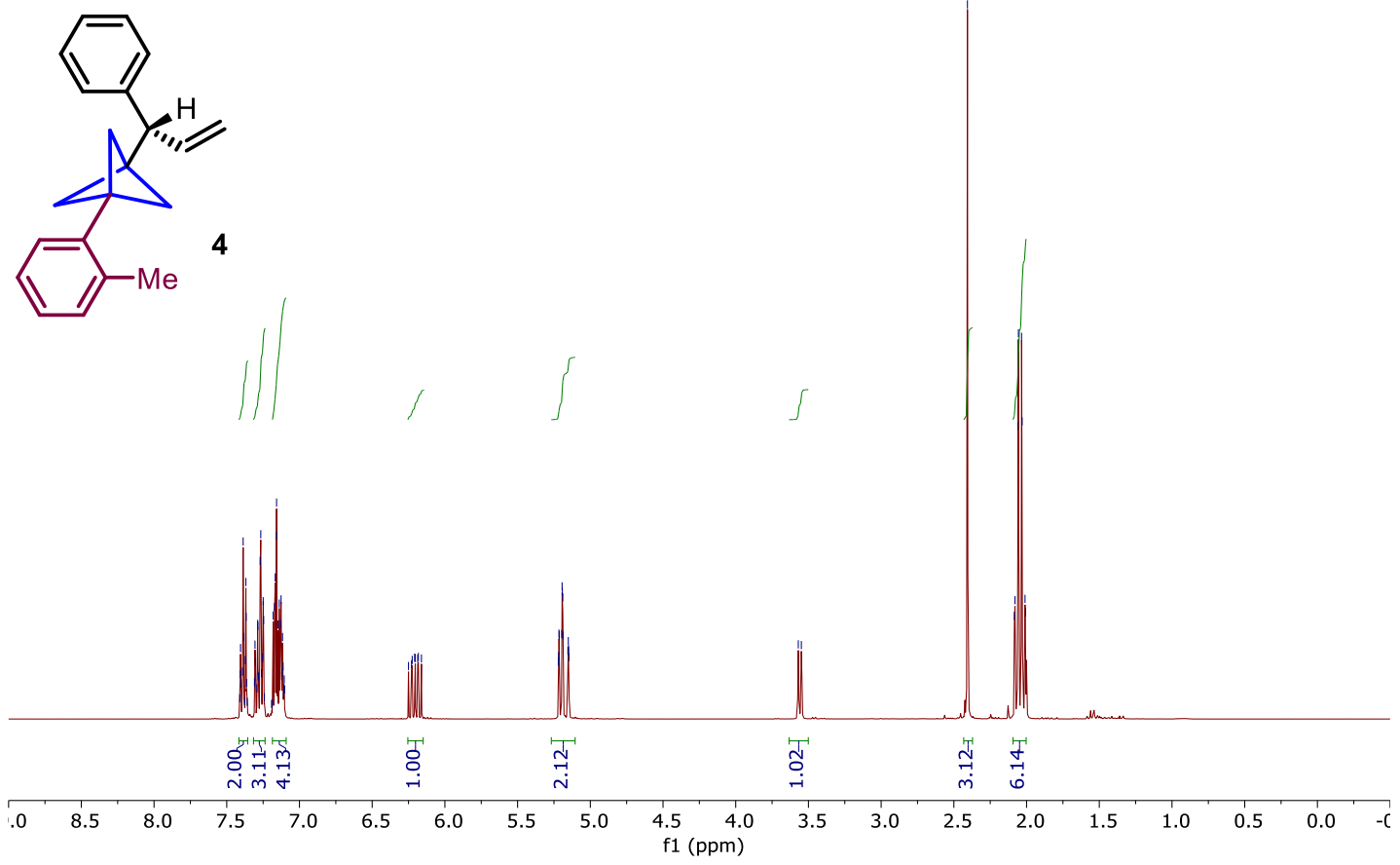

${ }^{13} \mathrm{C}$ NMR (100 MHz, $\left.\mathrm{CDCl}_{3}\right)$ spectrum for product 4 (see procedure):

54948 yu576.11.fid

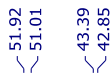

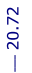
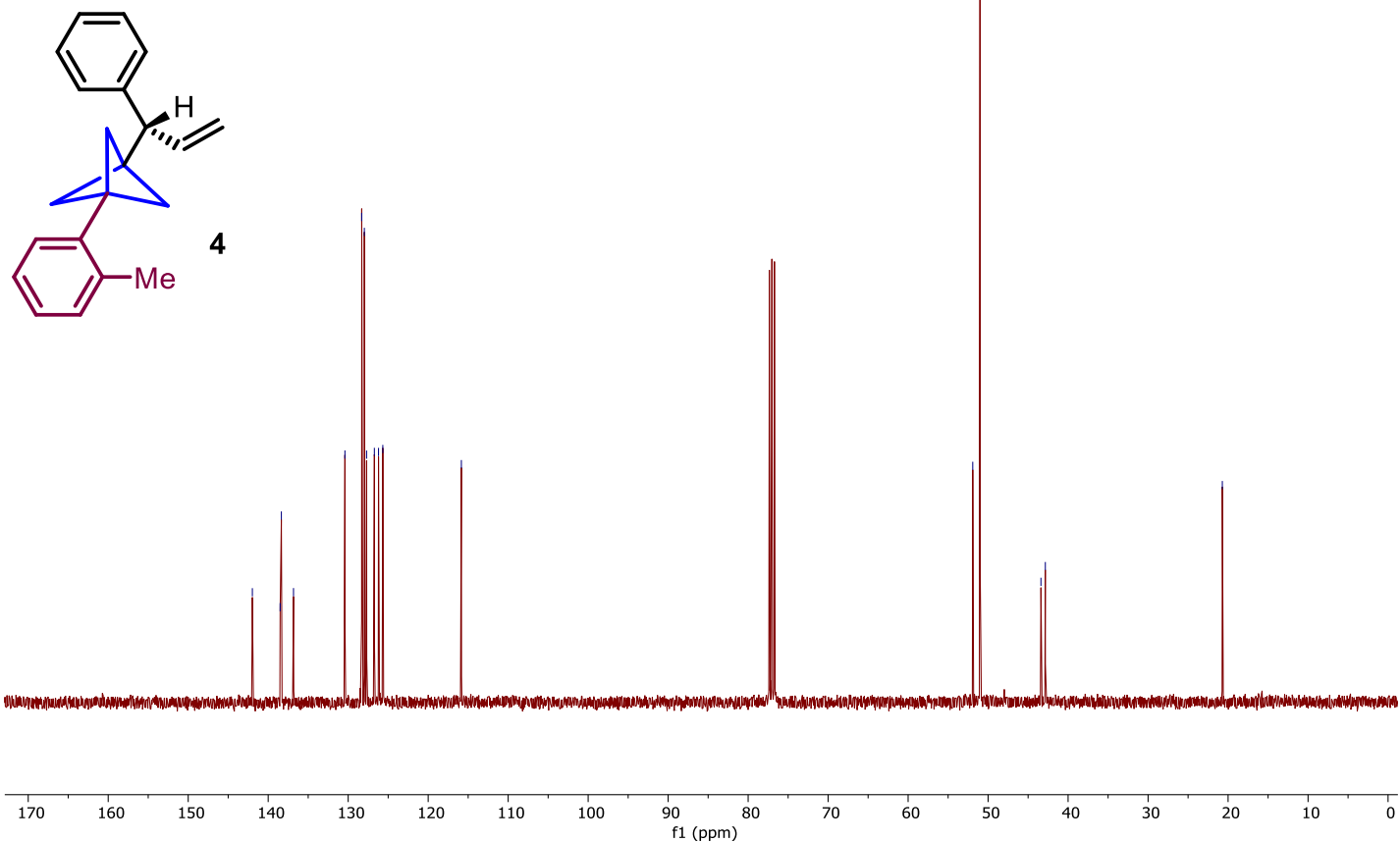

S68 
${ }^{1} \mathrm{H}$ NMR (400 MHz, $\left.\mathrm{CDCl}_{3}\right)$ spectrum for product 5 (see procedure):

58422 yu643.10.fid

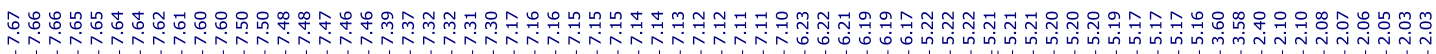
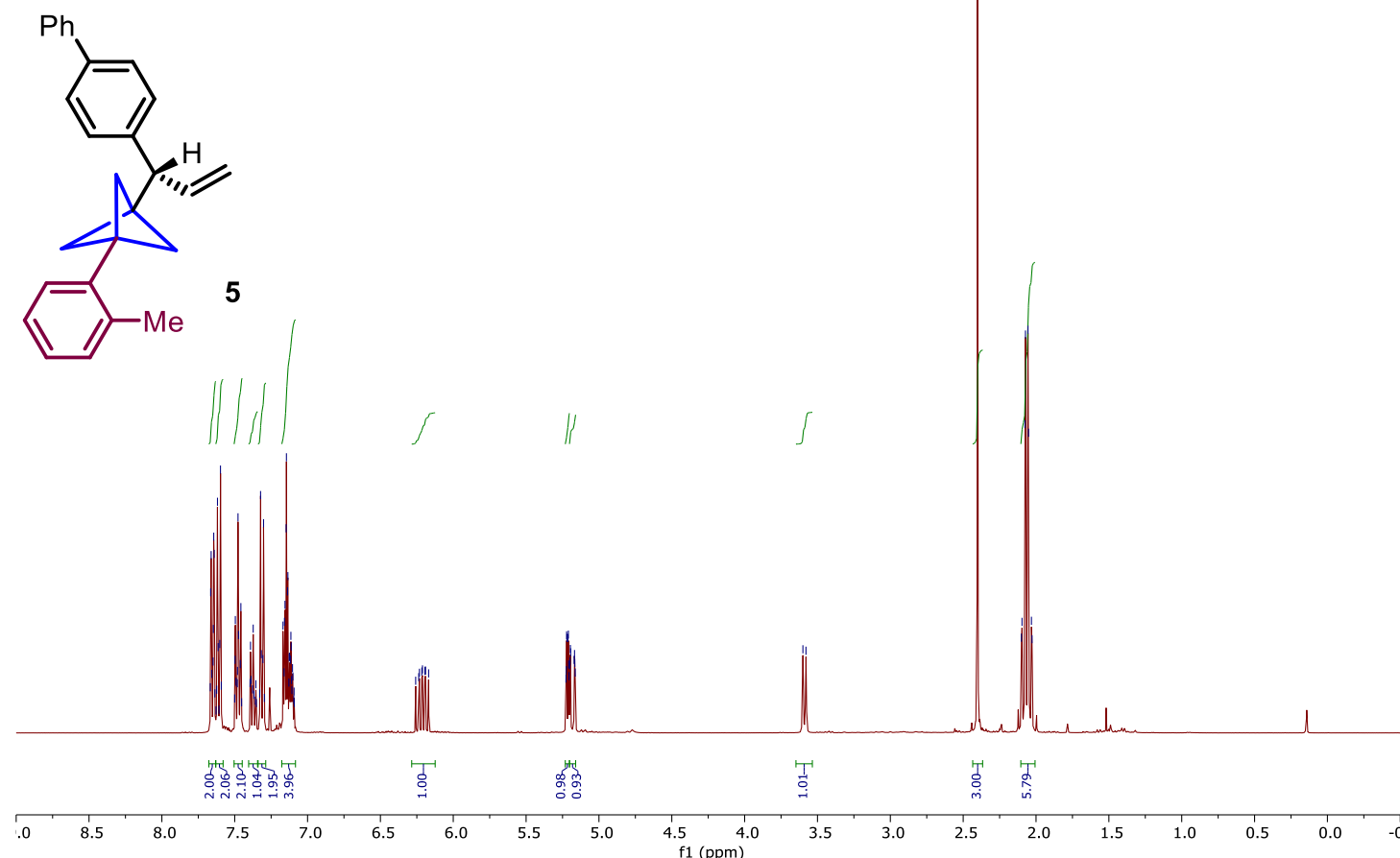

${ }^{13} \mathrm{C}$ NMR $\left(100 \mathrm{MHz}, \mathrm{CDCl}_{3}\right)$ spectrum for product 5 (see procedure):

58422 yu643.11.fid

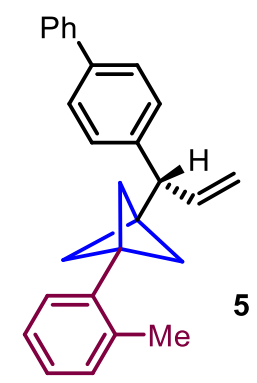

\section{5}

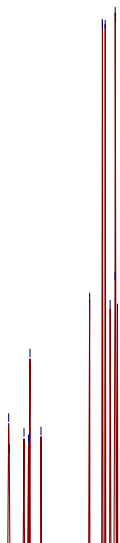


${ }^{1} \mathrm{H}$ NMR (400 MHz, $\mathrm{CDCl}_{3}$ ) spectrum for product 33 (see procedure):

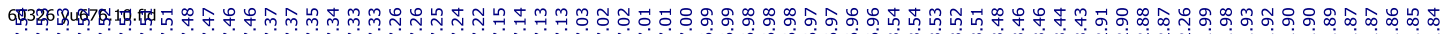
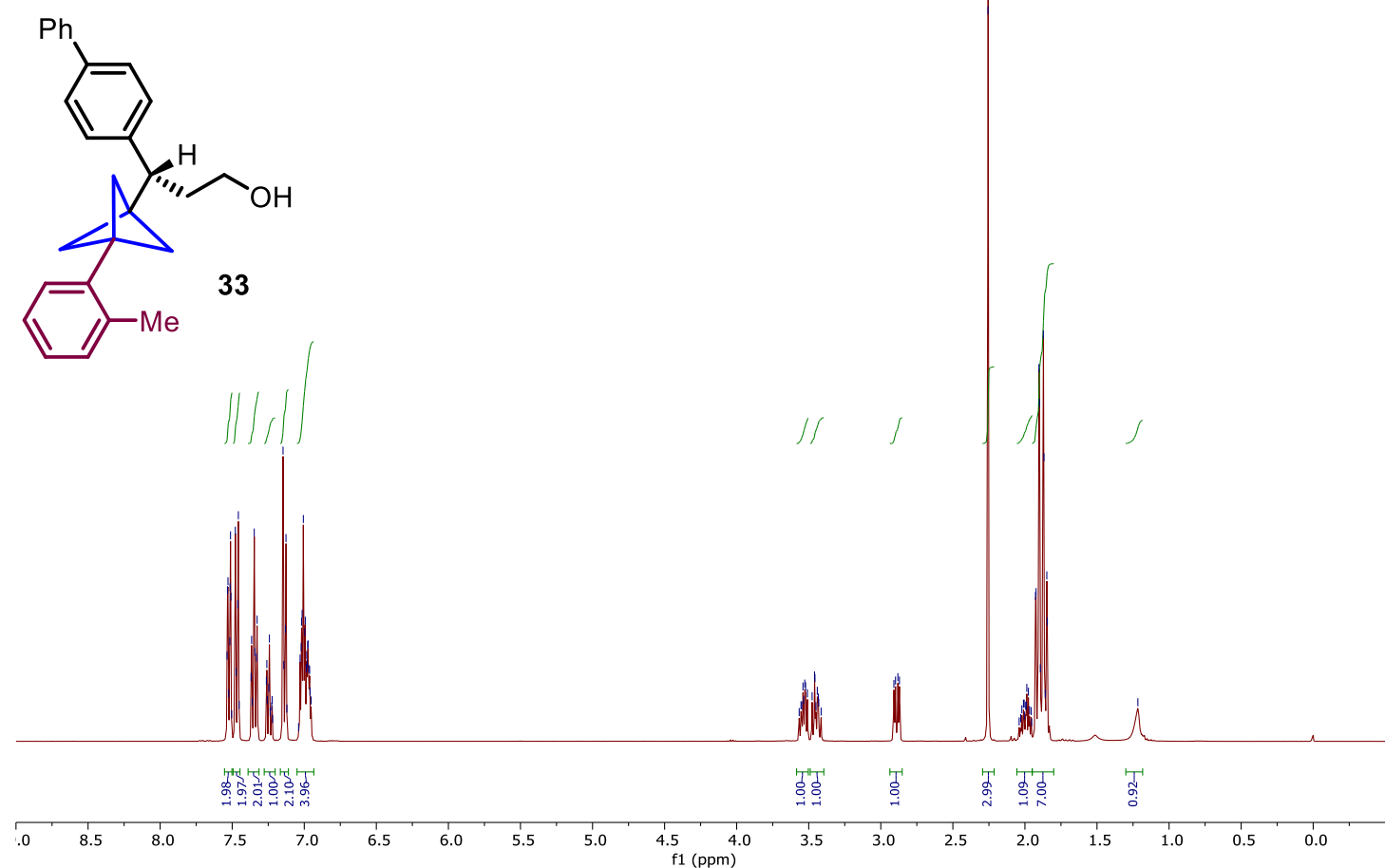

${ }^{13} \mathrm{C}$ NMR (100 MHz, $\left.\mathrm{CDCl}_{3}\right)$ spectrum for product 33 (see procedure):

60326 yu676.11.fid

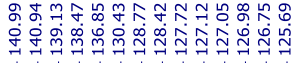

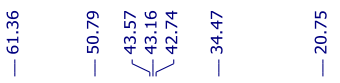<smiles>Cc1ccccc1C1(CCO)CCC1(CCO)c1ccc(-c2ccccc2)cc1</smiles> 
${ }^{1} \mathrm{H}$ NMR (400 MHz, $\mathrm{CDCl}_{3}$ ) spectrum for product 6 (see procedure):

60886.10.fid

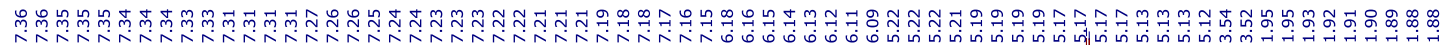

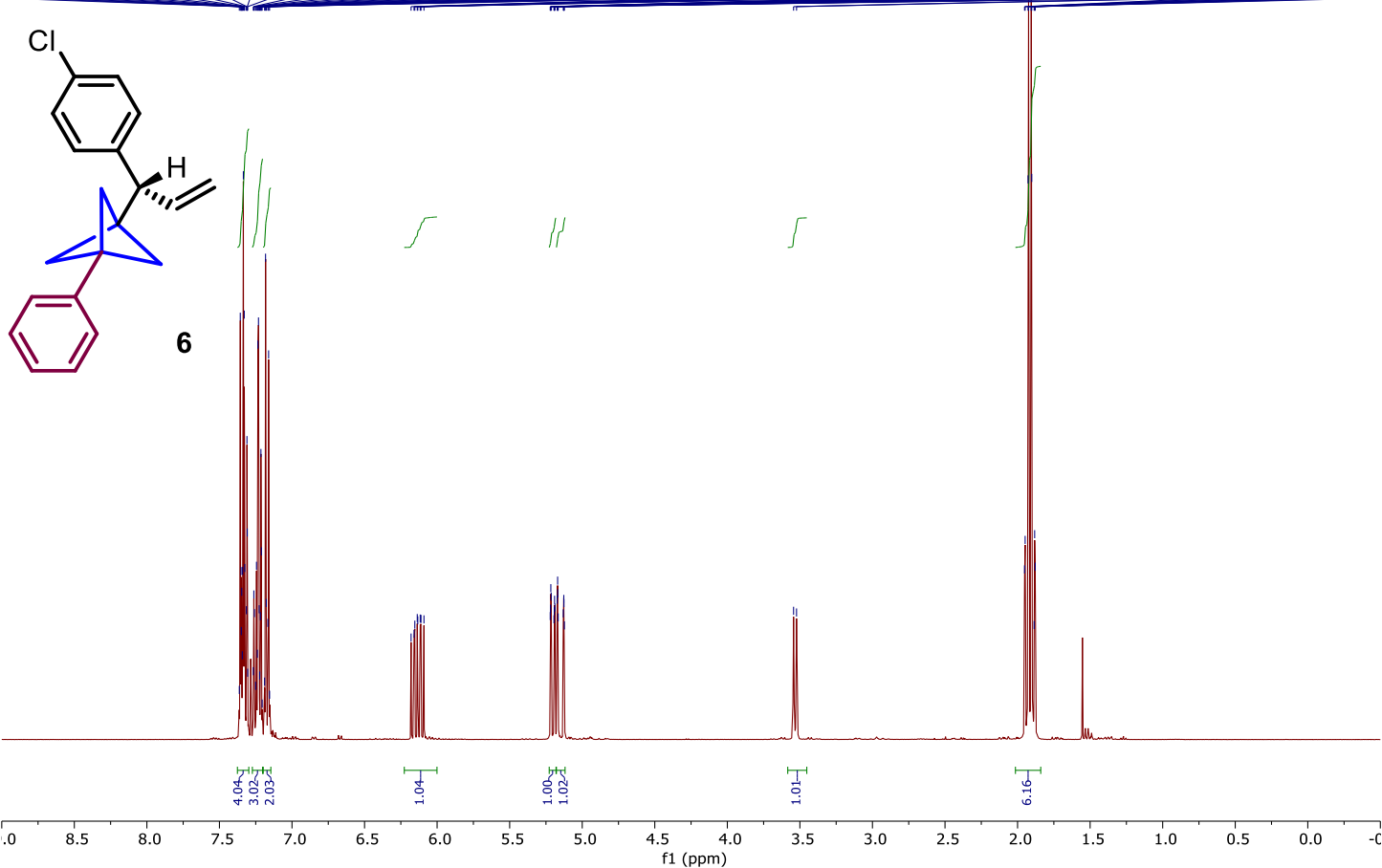

${ }^{13} \mathrm{C}$ NMR $\left(100 \mathrm{MHz}, \mathrm{CDCl}_{3}\right.$ ) spectrum for product 6 (see procedure):

60886.11.fid

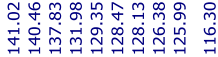

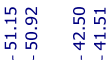

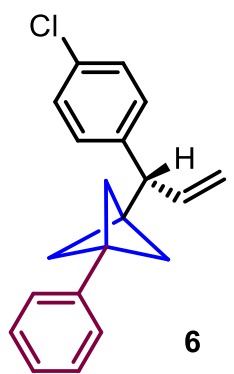

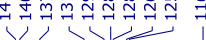

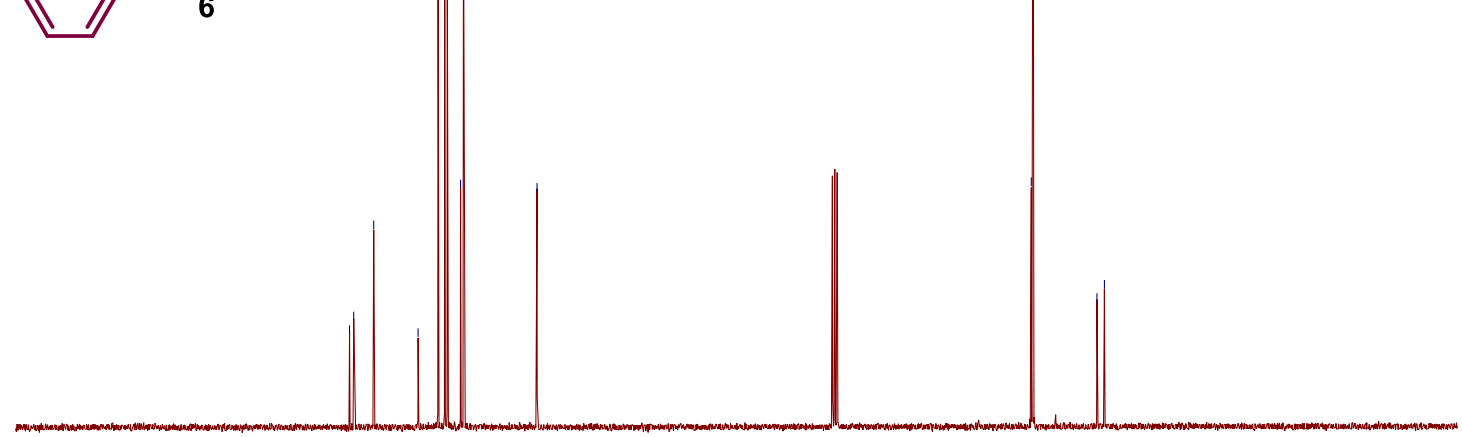


${ }^{1} \mathrm{H}$ NMR (400 MHz, $\mathrm{CDCl}_{3}$ ) spectrum for product 7 (see procedure):

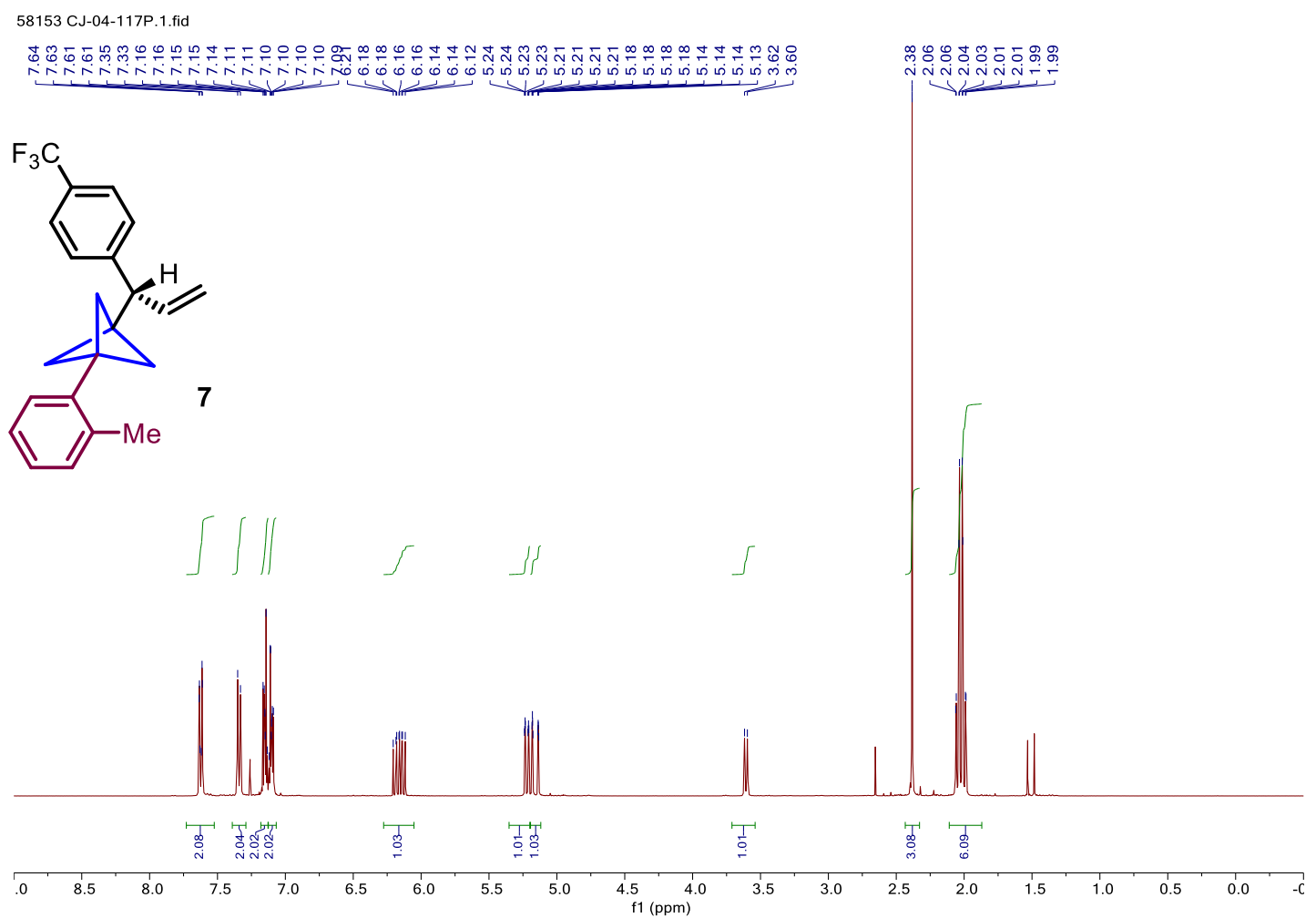

${ }^{19} \mathrm{~F}$ NMR $\left(377 \mathrm{MHz}, \mathrm{CDCl}_{3}\right.$ ) spectrum for product 7 (see procedure):

61592 CJ-04-117P.2.fid

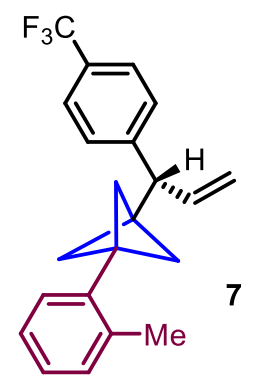

$\begin{array}{llllllllllllllllllllllllllllll}1 & 10 & 30 & 20 & 10 & 0 & -10 & -20 & -30 & -40 & -50 & -60 & -70 & -80 & -90 & -100 & -110 & -120 & -130 & -140 & -150 & -160 & -170 & -180 & -190 & -200\end{array}$ 
${ }^{13} \mathrm{C}$ NMR $\left(100 \mathrm{MHz}, \mathrm{CDCl}_{3}\right)$ spectrum for product 7 (see procedure):

58153 CJ-04-117P.10.fid
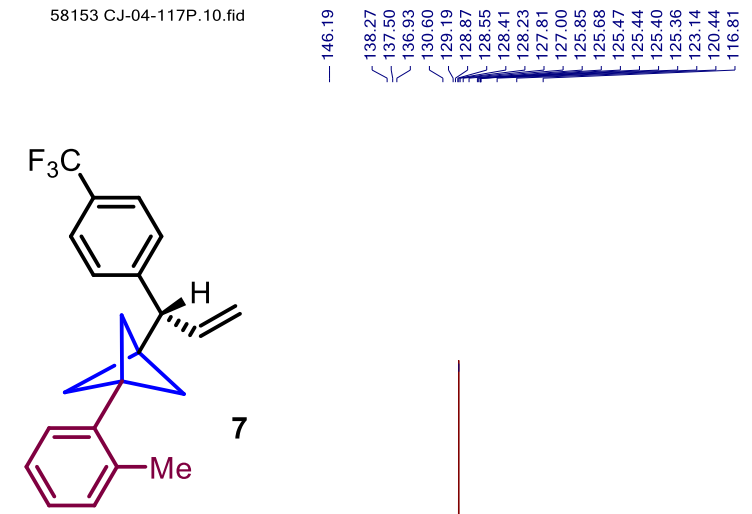

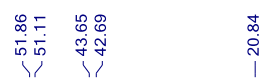

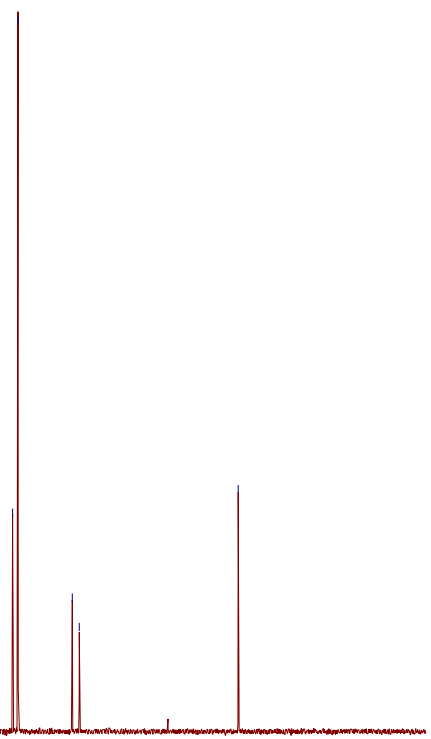

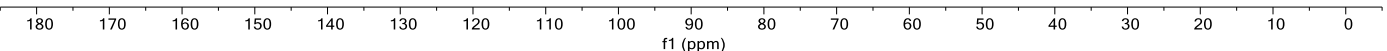


${ }^{1} \mathrm{H}$ NMR (400 MHz, $\mathrm{CDCl}_{3}$ ) spectrum for product 8 (see procedure):

59030 yu652.10.fid

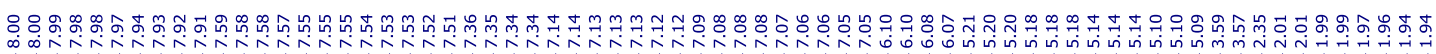
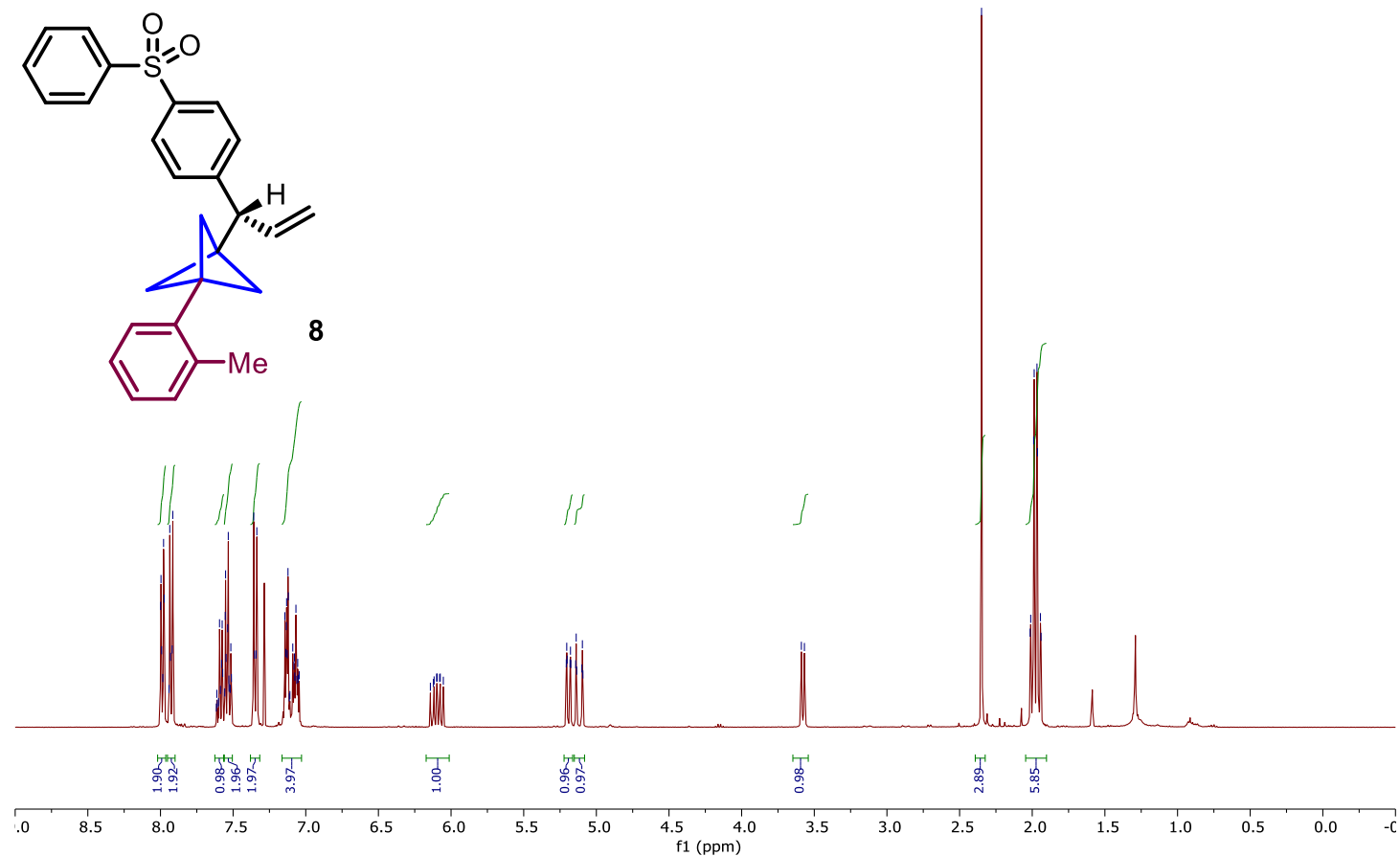

${ }^{13} \mathrm{C} \mathrm{NMR}\left(100 \mathrm{MHz}, \mathrm{CDCl}_{3}\right)$ spectrum for product 8 (see procedure):
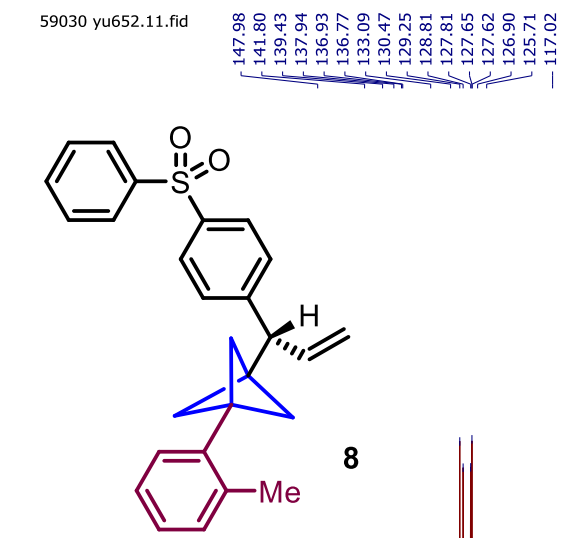

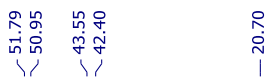

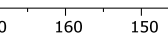

140

$30 \quad 120$

100

$\stackrel{90}{90}$ 
${ }^{1} \mathrm{H}$ NMR (400 MHz, $\mathrm{CDCl}_{3}$ ) spectrum for product $8 \mathbf{a}$ (see procedure):

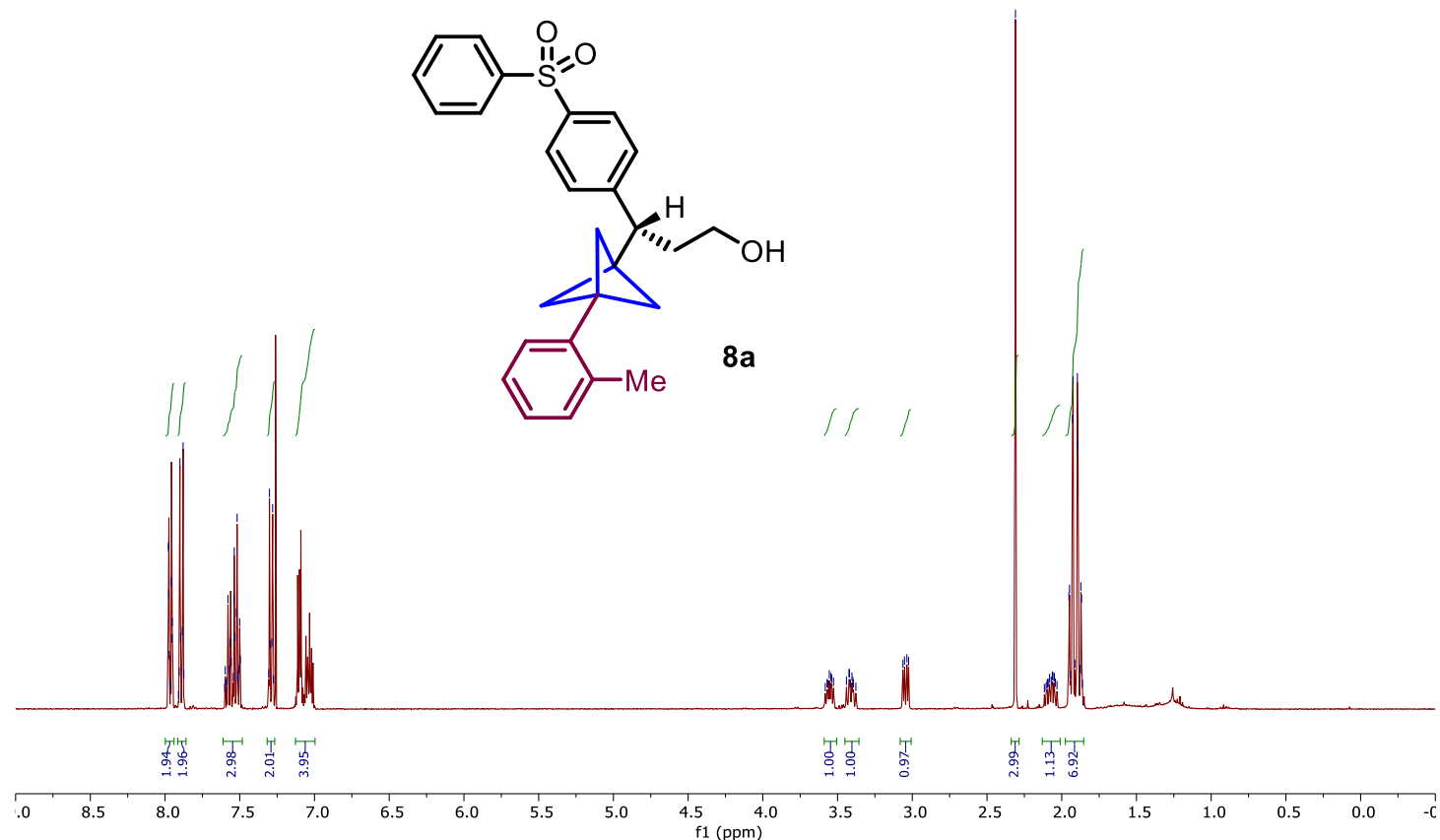

${ }^{13} \mathrm{C}$ NMR (100 MHz, $\mathrm{CDCl}_{3}$ ) spectrum for product $8 \mathbf{a}$ (see procedure):

va/dn18877yu677
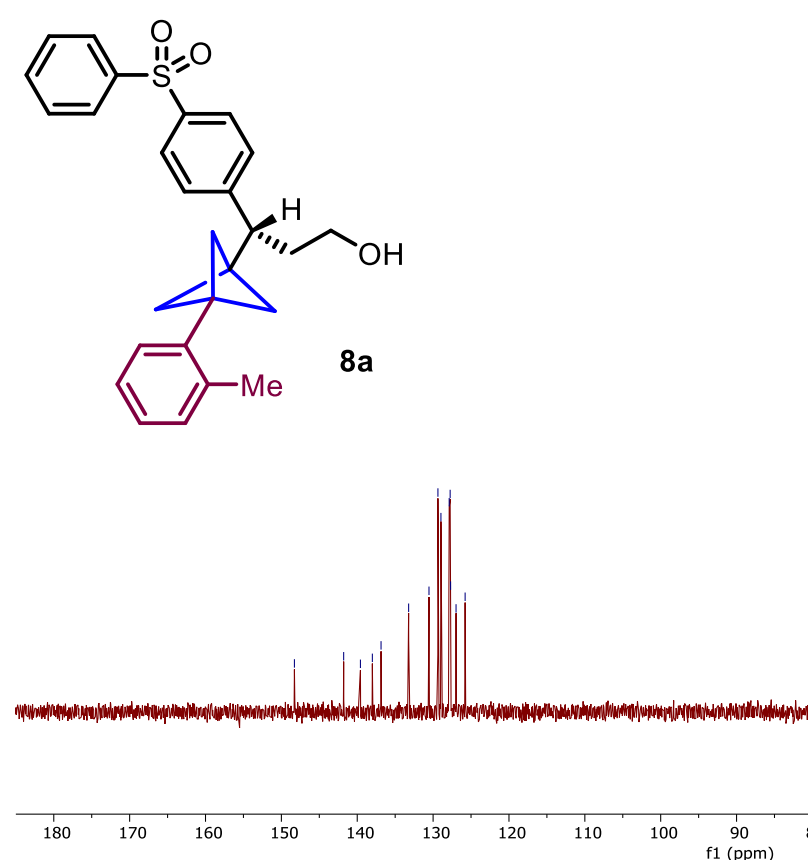
${ }^{1} \mathrm{H}$ NMR (400 MHz, $\mathrm{CDCl}_{3}$ ) spectrum for product 9 (see procedure):

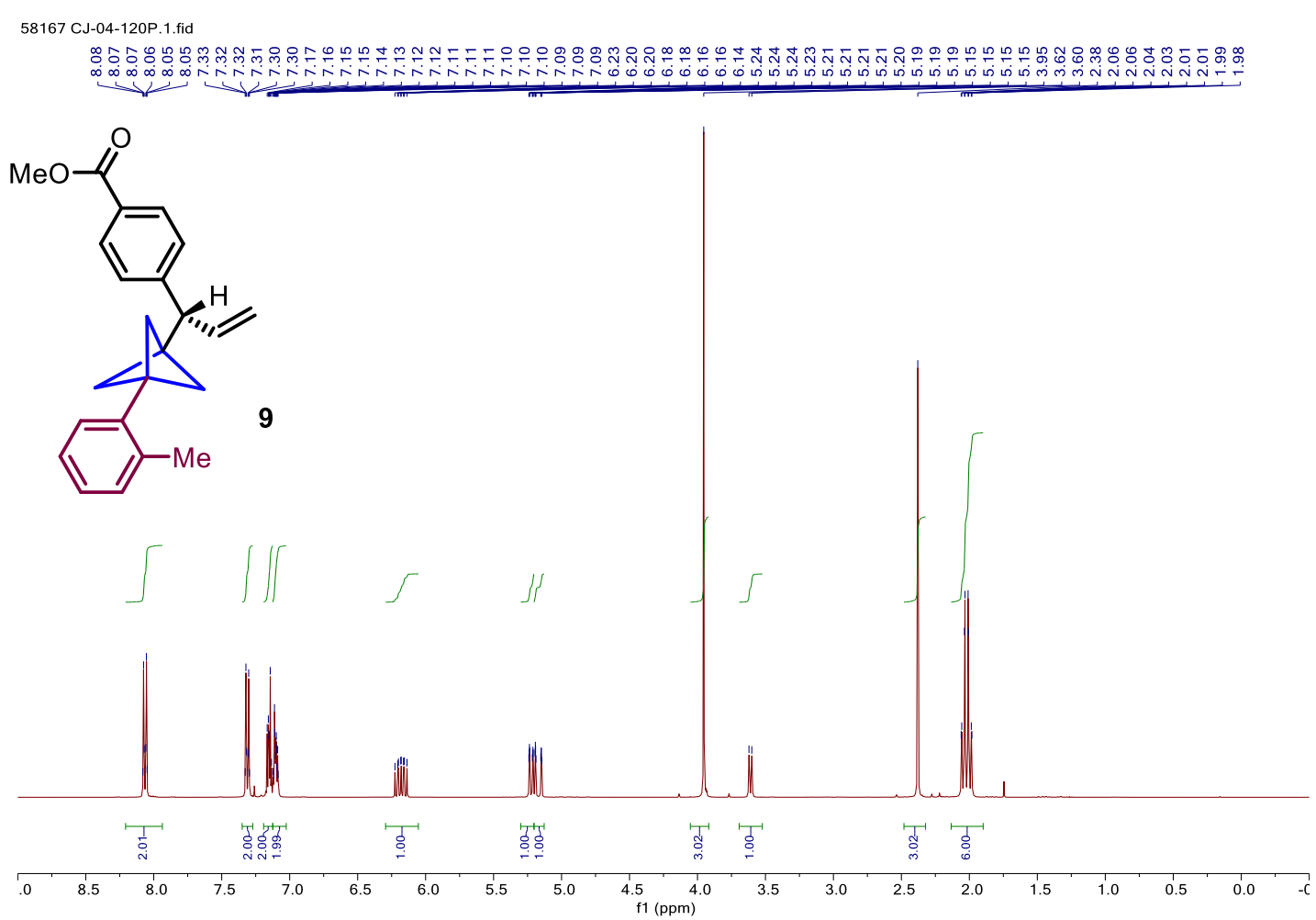

${ }^{13} \mathrm{C}$ NMR (100 MHz, $\mathrm{CDCl}_{3}$ ) spectrum for product 9 (see procedure):
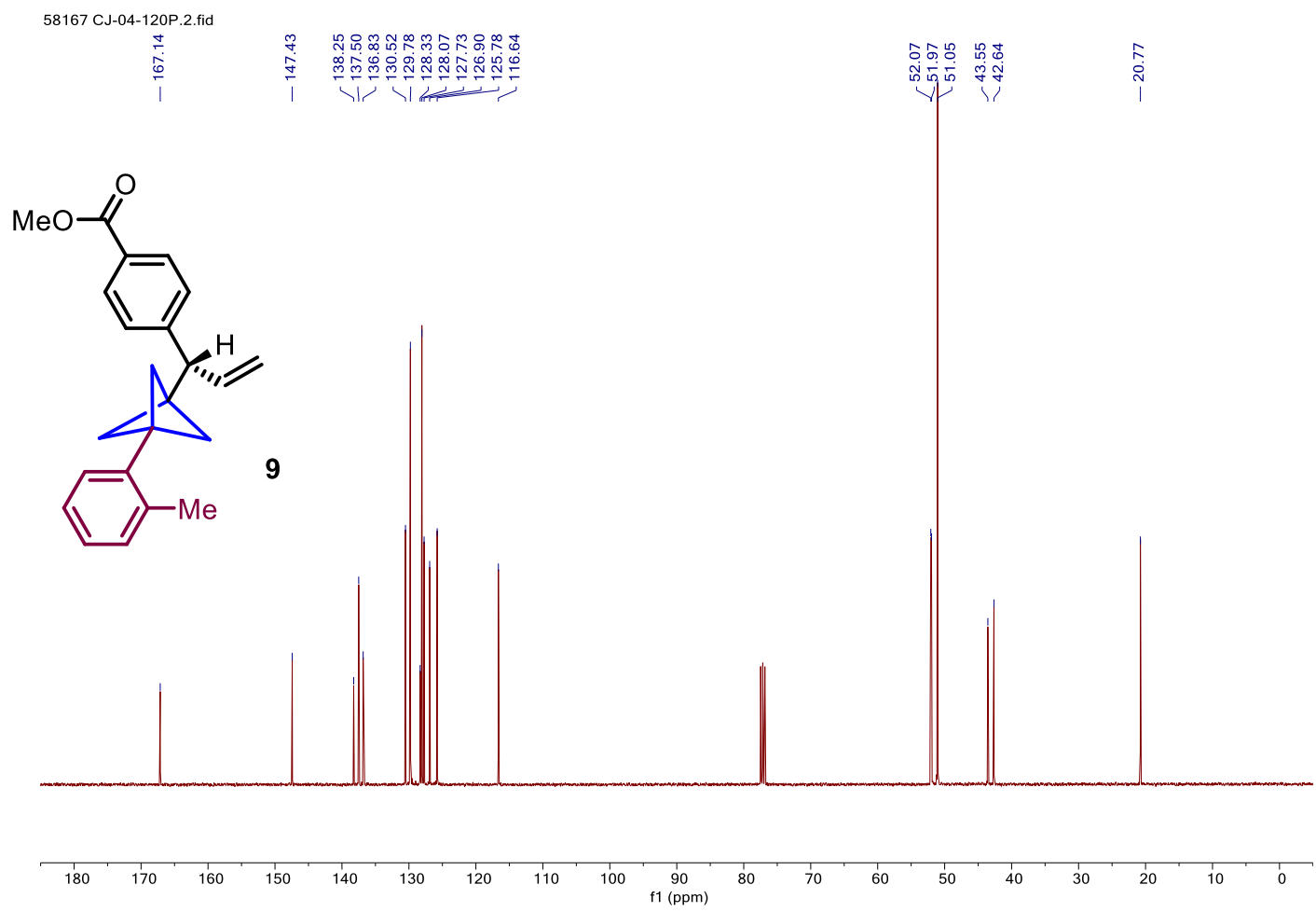
${ }^{1} \mathrm{H}$ NMR (400 MHz, $\mathrm{CDCl}_{3}$ ) spectrum for product $\mathbf{1 0}$ (see procedure):

28590 CJ-04-123P.1.fid

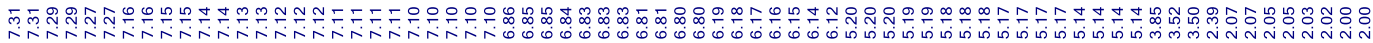
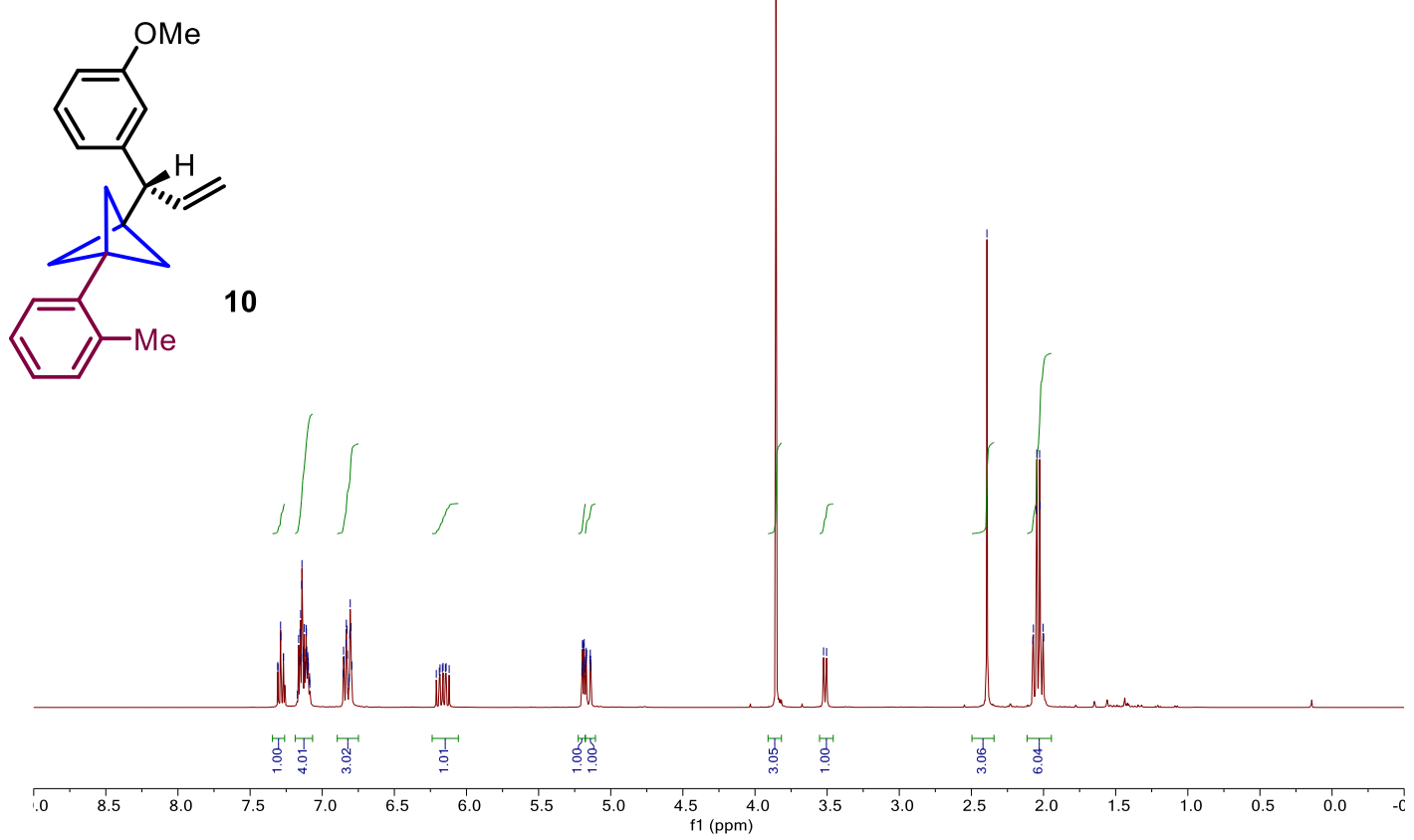

${ }^{13} \mathrm{C} \mathrm{NMR}\left(100 \mathrm{MHz}, \mathrm{CDCl}_{3}\right)$ spectrum for product $\mathbf{1 0}$ (see procedure):
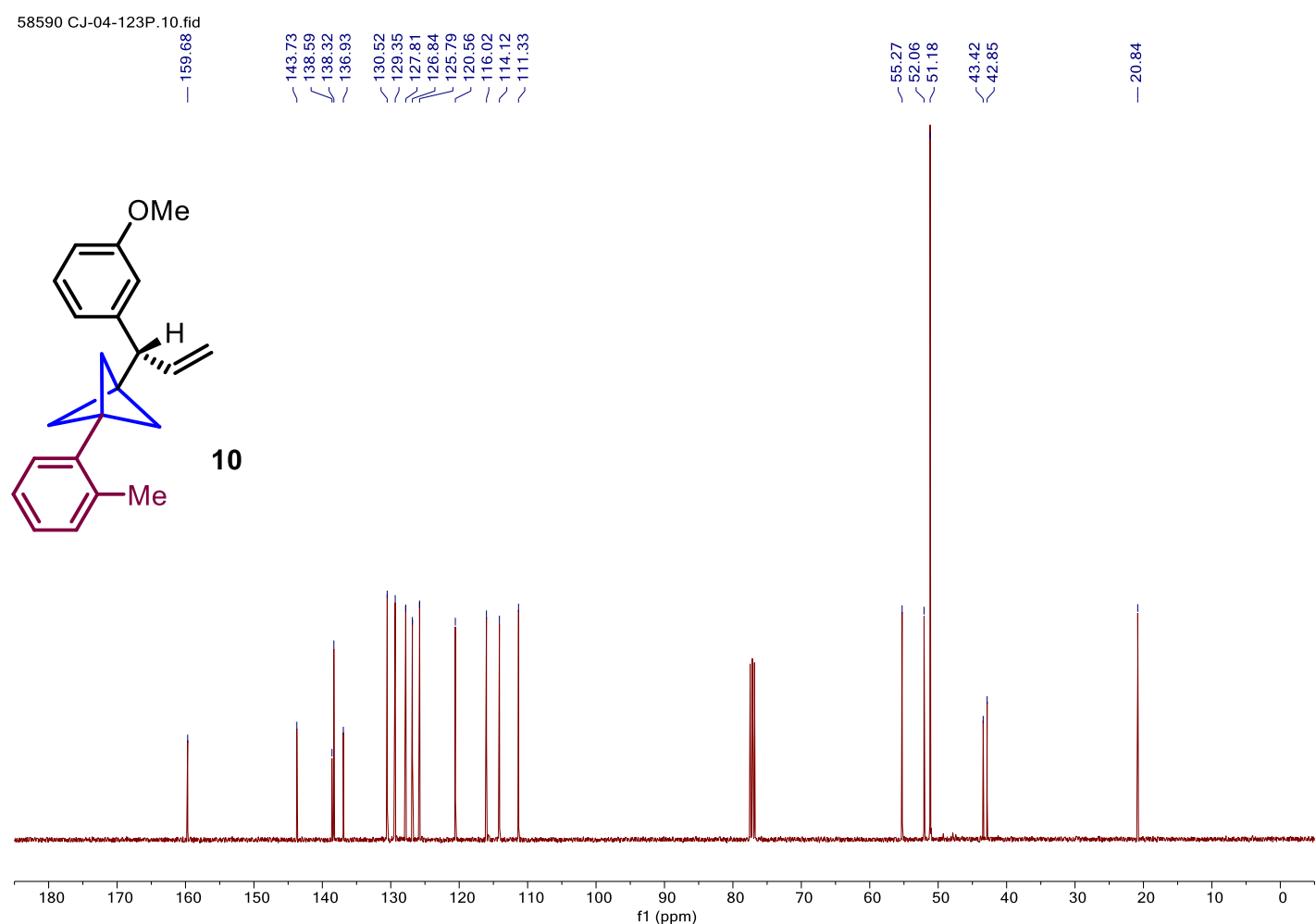
${ }^{1} \mathrm{H}$ NMR (400 MHz, $\mathrm{CDCl}_{3}$ ) spectrum for product 11 (see procedure): 58168 CJ-04-119P.1.fid

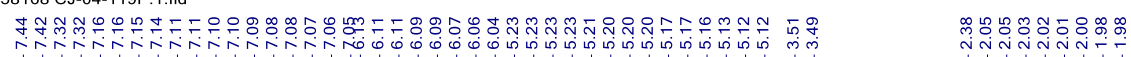<smiles>C=C[C@H](c1ccc(Cl)c(Cl)c1)C12CCC(c3ccccc3C)(C1)C2</smiles>
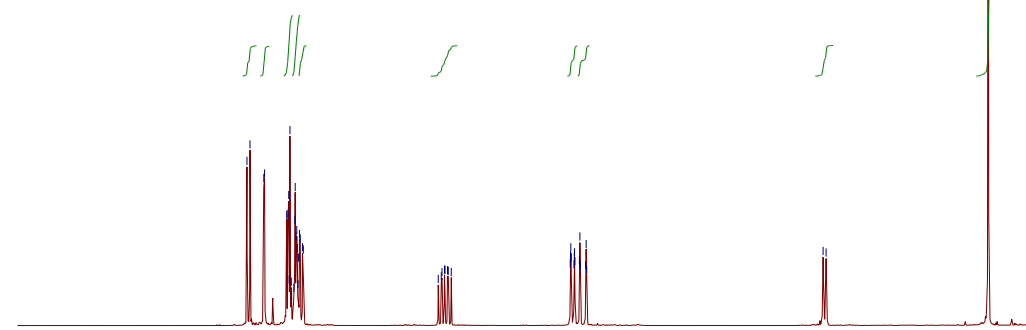

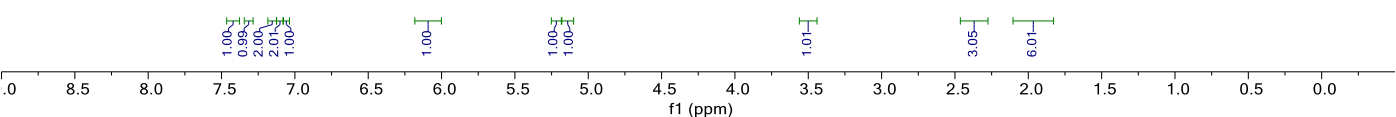

${ }^{13} \mathrm{C} \mathrm{NMR}\left(100 \mathrm{MHz}, \mathrm{CDCl}_{3}\right)$ spectrum for product 11 (see procedure):
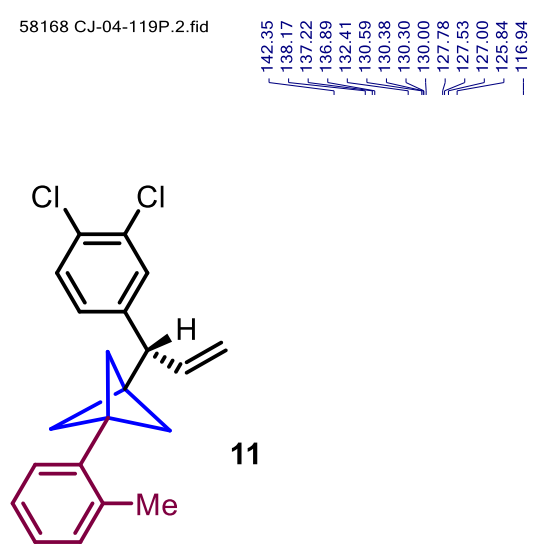

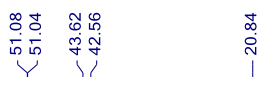

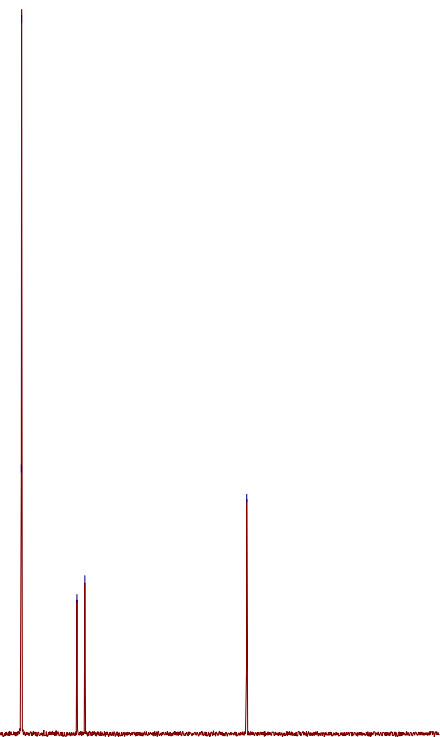

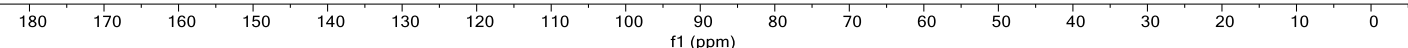


${ }^{1} \mathrm{H}$ NMR (400 MHz, $\mathrm{CDCl}_{3}$ ) spectrum for product 12 (see procedure):

55888 yu603.10.fid

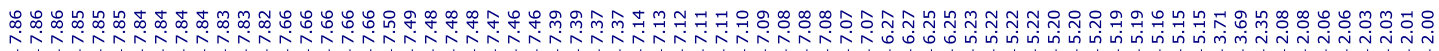
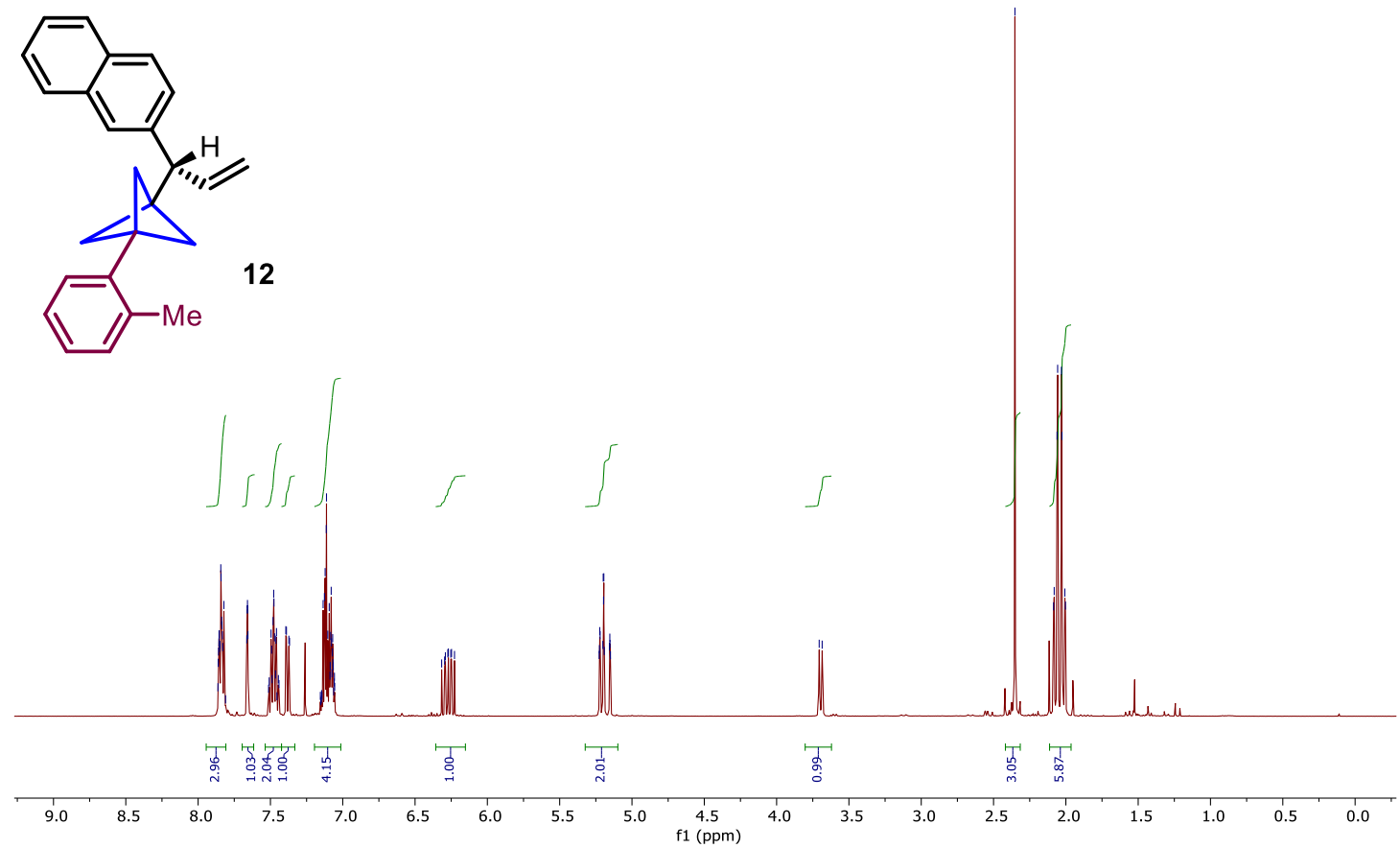

${ }^{13} \mathrm{C} \mathrm{NMR}\left(100 \mathrm{MHz}, \mathrm{CDCl}_{3}\right)$ spectrum for product 12 (see procedure):

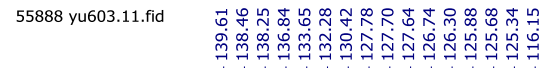

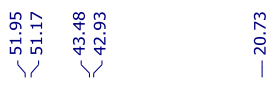

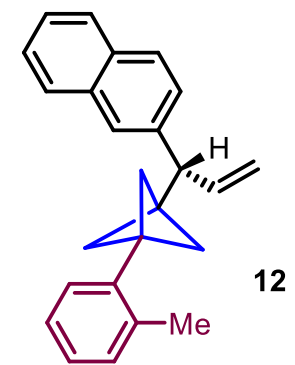

12

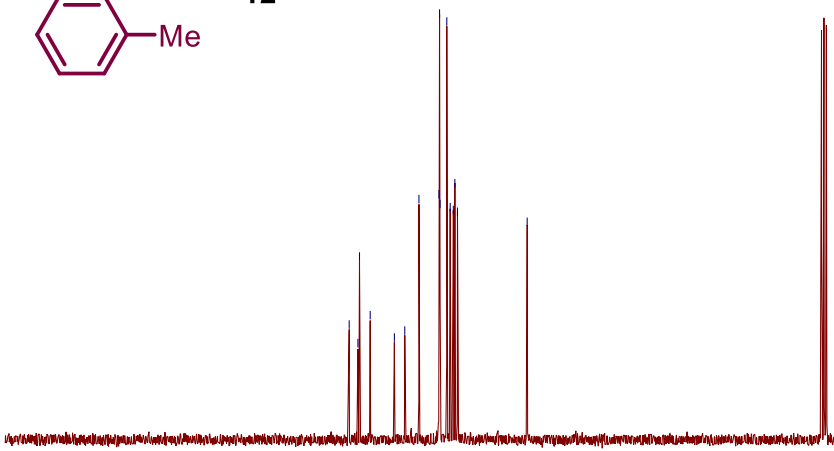

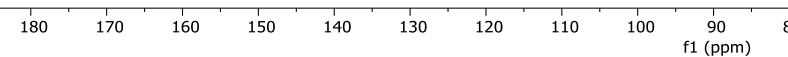


${ }^{1} \mathrm{H}$ NMR (400 MHz, $\mathrm{CDCl}_{3}$ ) spectrum for product 13 (see procedure):

59099 CJ-04-130P.1.fid

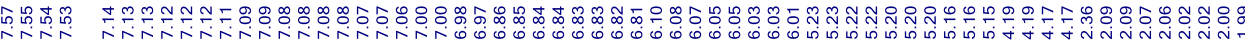<smiles>C=C[C@]1(c2ccc(F)cc2Br)CC2(c3ccccc3C)CCC1C2</smiles>

13

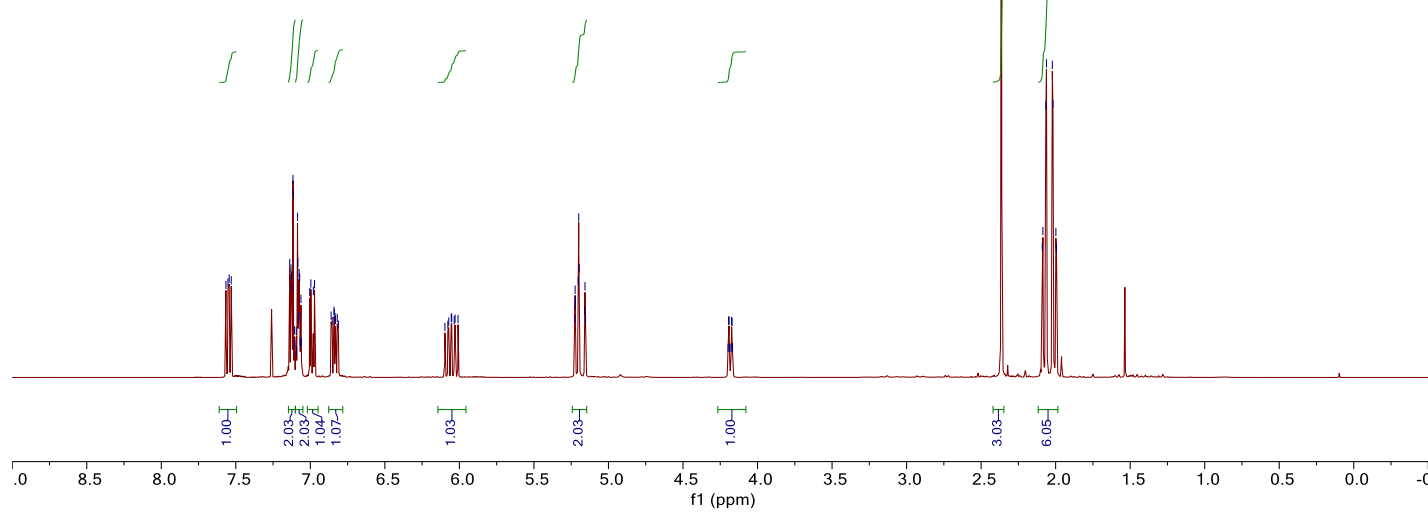

${ }^{19} \mathrm{~F} \mathrm{NMR}\left(377 \mathrm{MHz}, \mathrm{CDCl}_{3}\right.$ ) spectrum for product 13 (see procedure):

61593 CJ-04-130P.2.fid

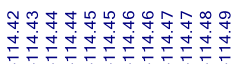

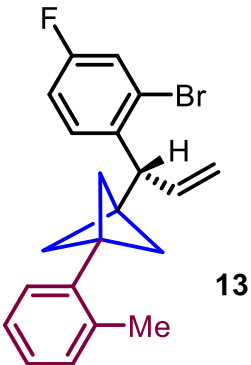

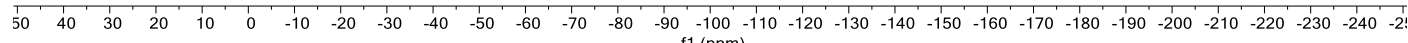


${ }^{13} \mathrm{C} \mathrm{NMR}\left(100 \mathrm{MHz}, \mathrm{CDCl}_{3}\right.$ ) spectrum for product 13 (see procedure):
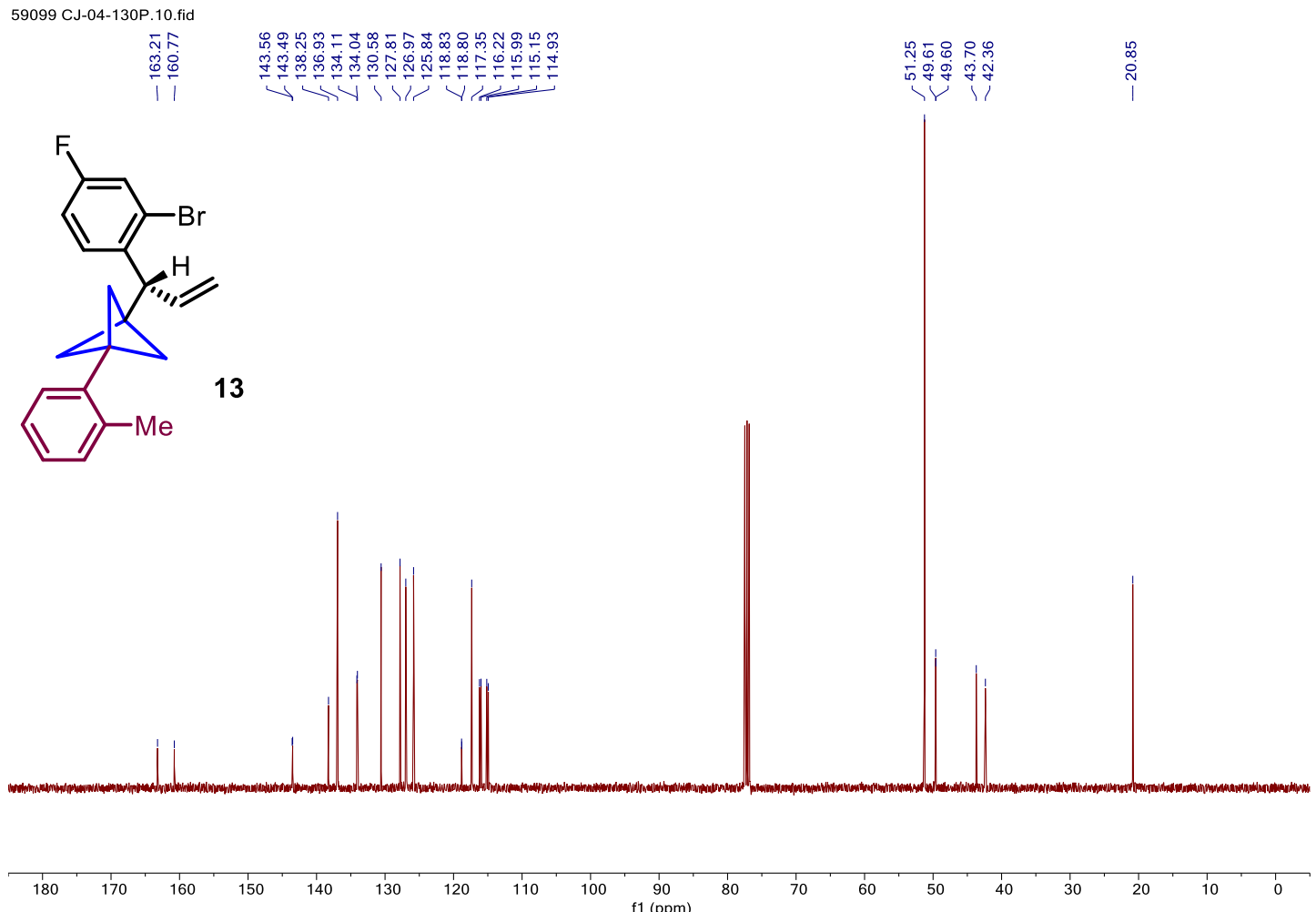
${ }^{1} \mathrm{H}$ NMR (400 MHz, $\mathrm{CDCl}_{3}$ ) spectrum for product $\mathbf{1 3 a}$ (see procedure):

59759 CJ-04-133P.1.fid

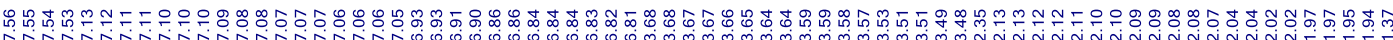<smiles>Cc1ccccc1C12CCC(CCO)(C1)C[C@]2(CCO)c1ccc(F)cc1Br</smiles>
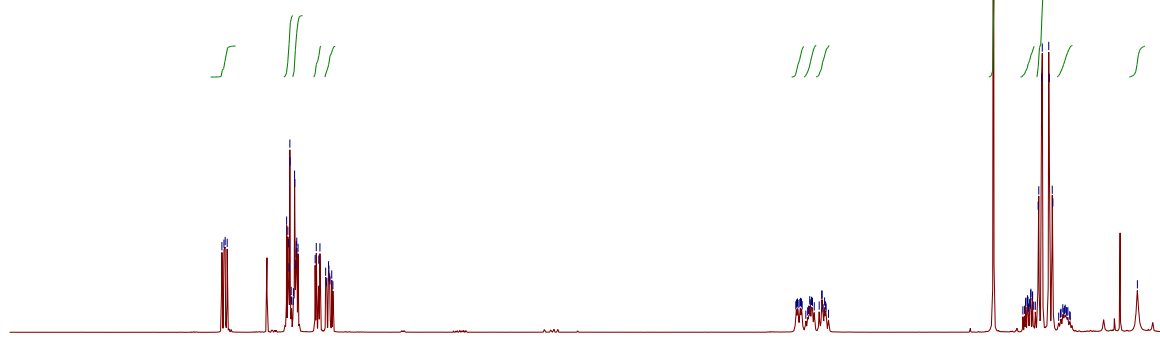

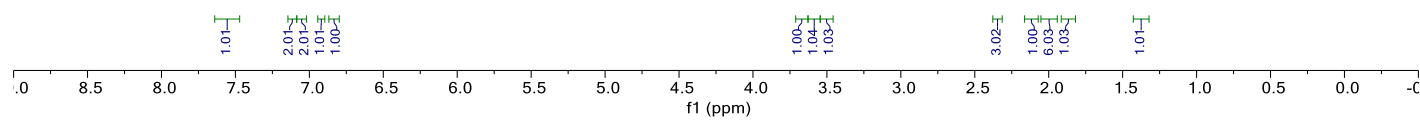

${ }^{19} \mathrm{~F}$ NMR (377 MHz, $\mathrm{CDCl}_{3}$ ) spectrum for product 13a (see procedure):

59995 CJ-04-133P.2.fid

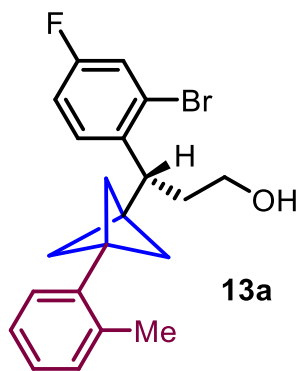

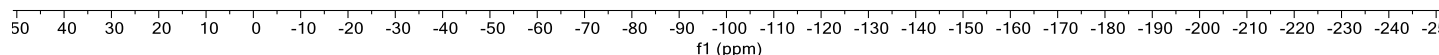


${ }^{13} \mathrm{C} \mathrm{NMR}\left(100 \mathrm{MHz}, \mathrm{CDCl}_{3}\right)$ spectrum for product $\mathbf{1 3 a}$ (see procedure): 59759 CJ-04-133P.10.fid

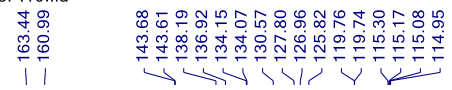<smiles>Cc1ccccc1C1(CCO)CCCC1(CCO)c1ccc(F)cc1Br</smiles>

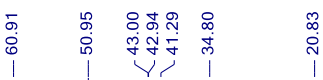

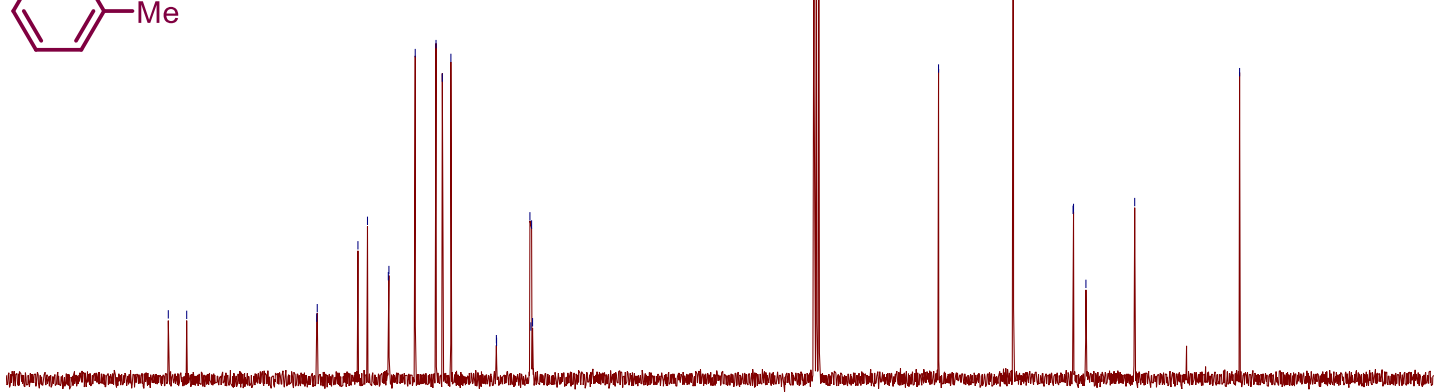

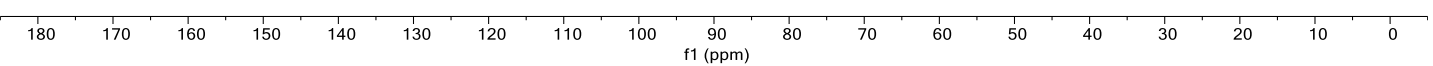


${ }^{1} \mathrm{H}$ NMR (400 MHz, $\mathrm{CDCl}_{3}$ ) spectrum for product 14 (see procedure):

57260 yu630.10.fid

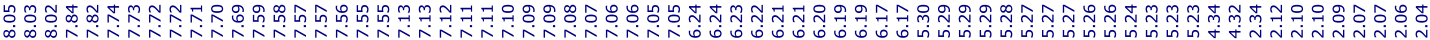

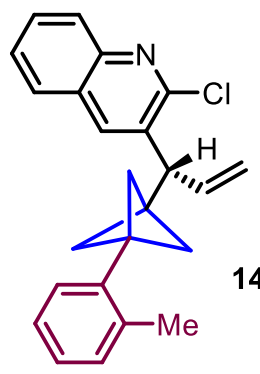

14
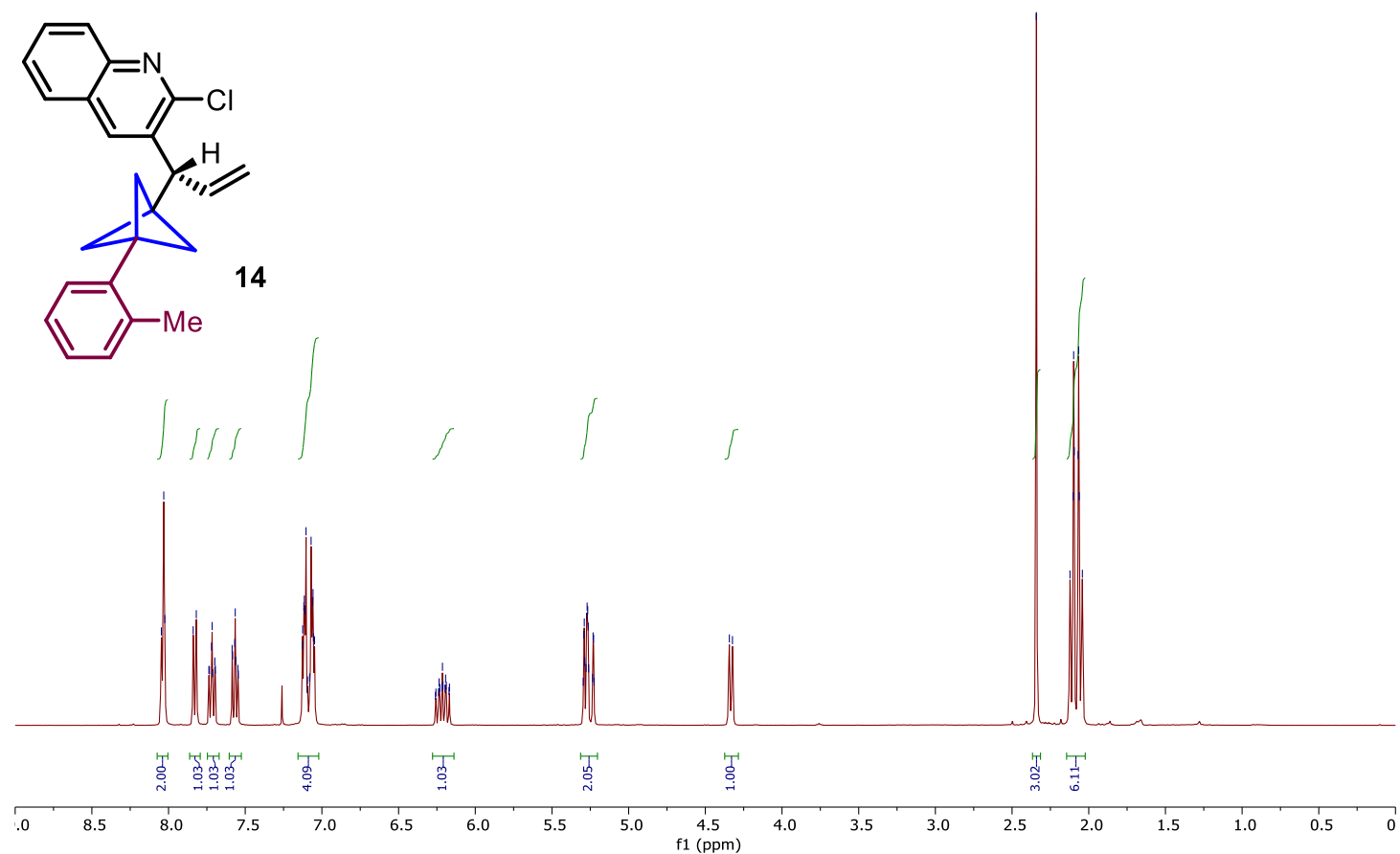

${ }^{13} \mathrm{C}$ NMR (100 MHz, $\mathrm{CDCl}_{3}$ ) spectrum for product 14 (see procedure):

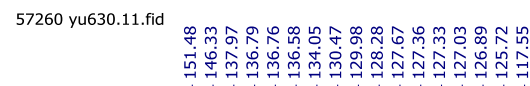

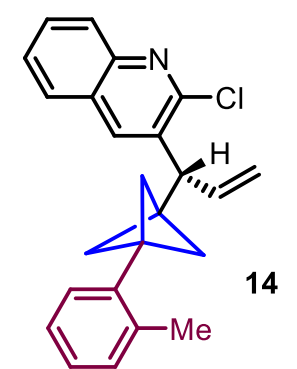

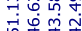

$\stackrel{\substack{n \\ \stackrel{1}{i}}}{1}$

14

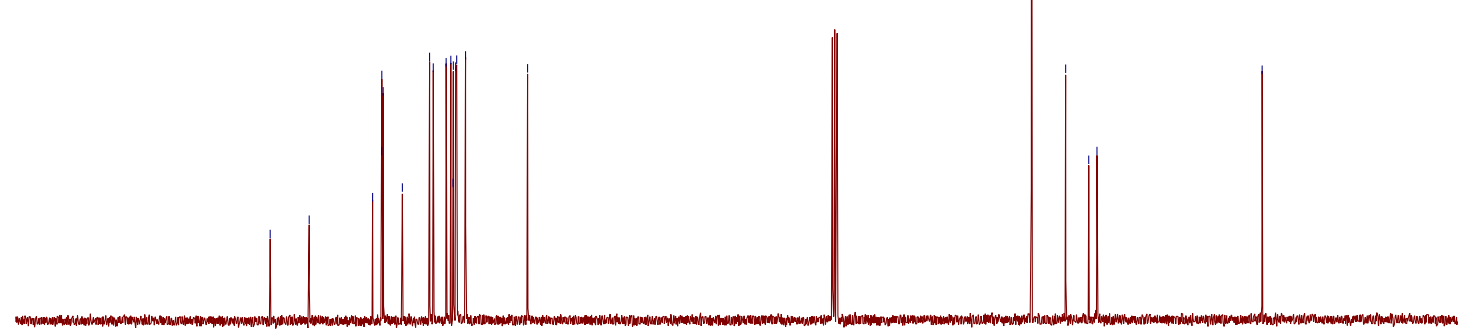

180

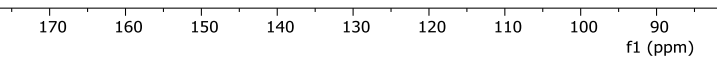


${ }^{1} \mathrm{H}$ NMR (400 MHz, $\mathrm{CDCl}_{3}$ ) spectrum for product 15 (see procedure):

61061 yu704.10.fid

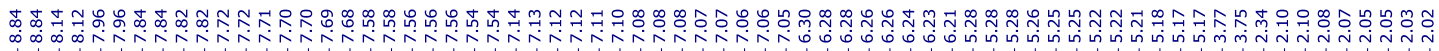

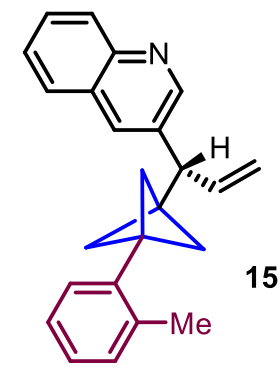

15
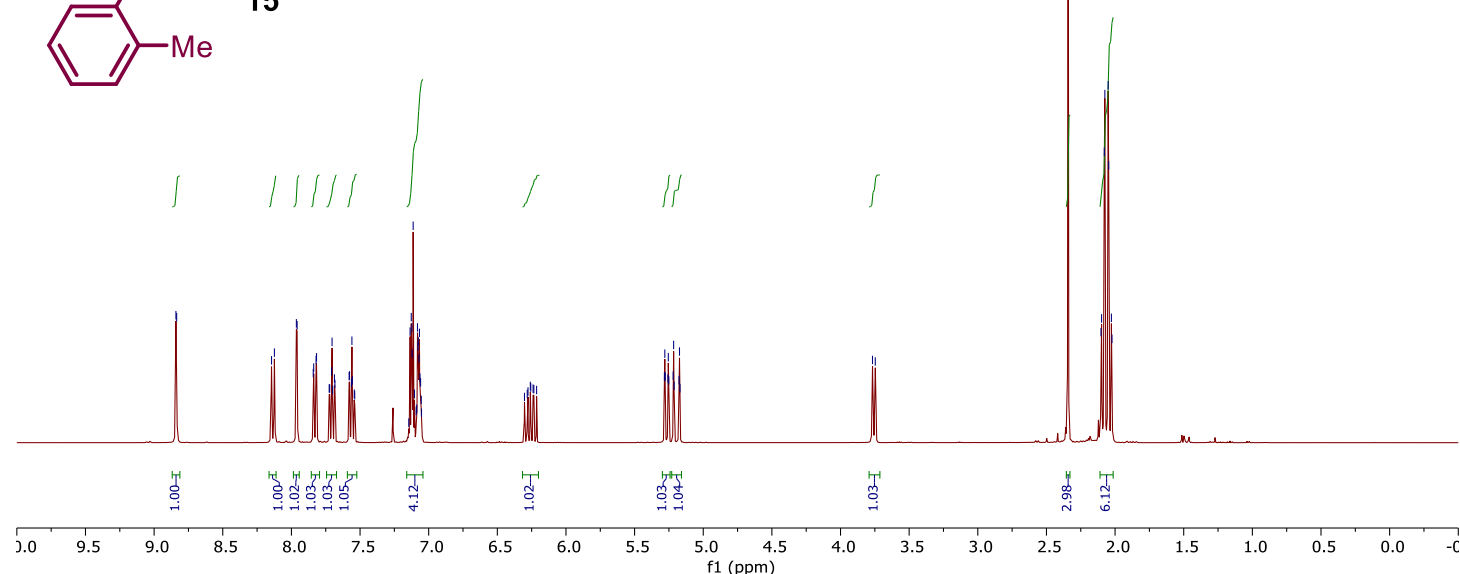

${ }^{13} \mathrm{C} \mathrm{NMR}\left(100 \mathrm{MHz}, \mathrm{CDCl}_{3}\right)$ spectrum for product 15 (see procedure):

61061 yu704.11.fid

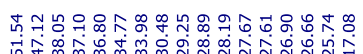

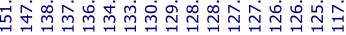
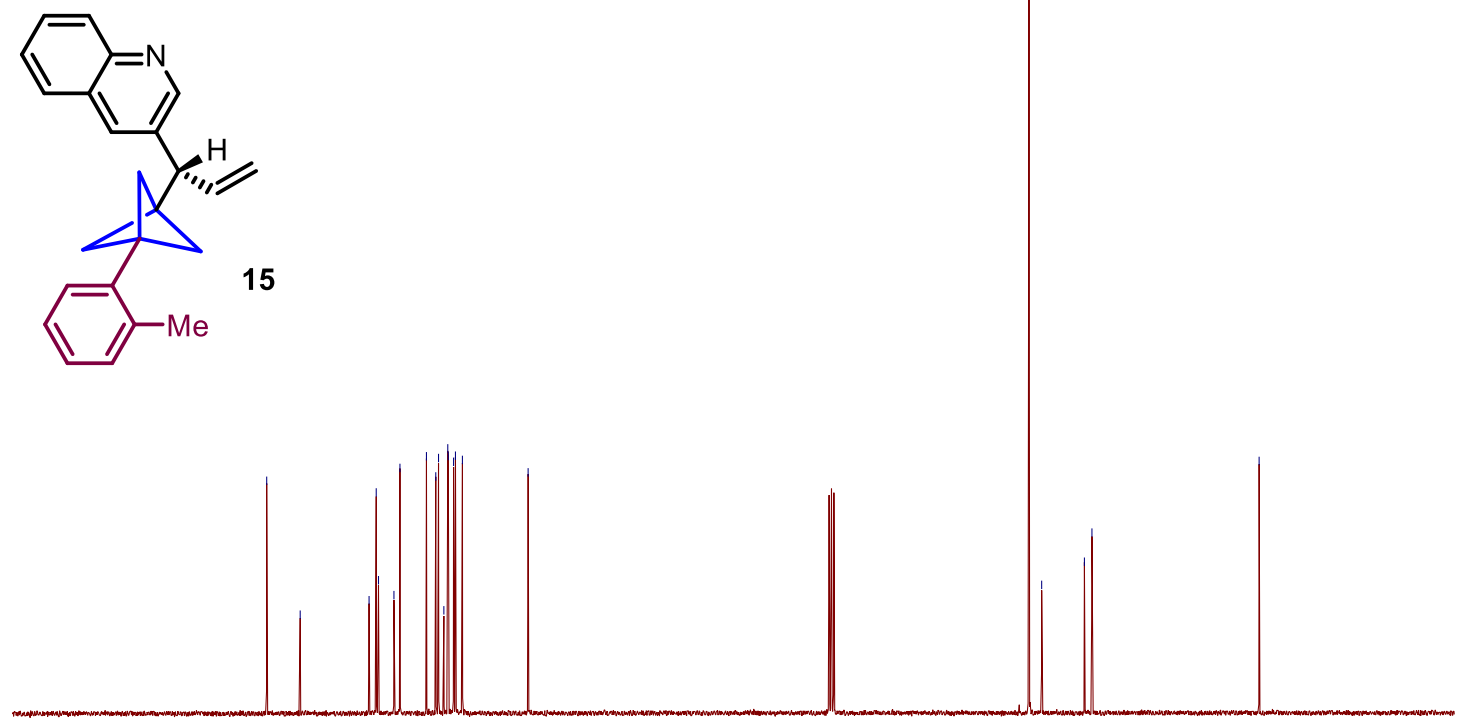
${ }^{1} \mathrm{H}$ NMR (400 MHz, $\mathrm{CDCl}_{3}$ ) spectrum for product 16 (see procedure):

58337 yu637.10.fid

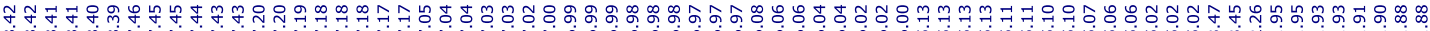

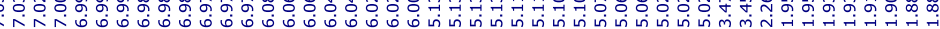
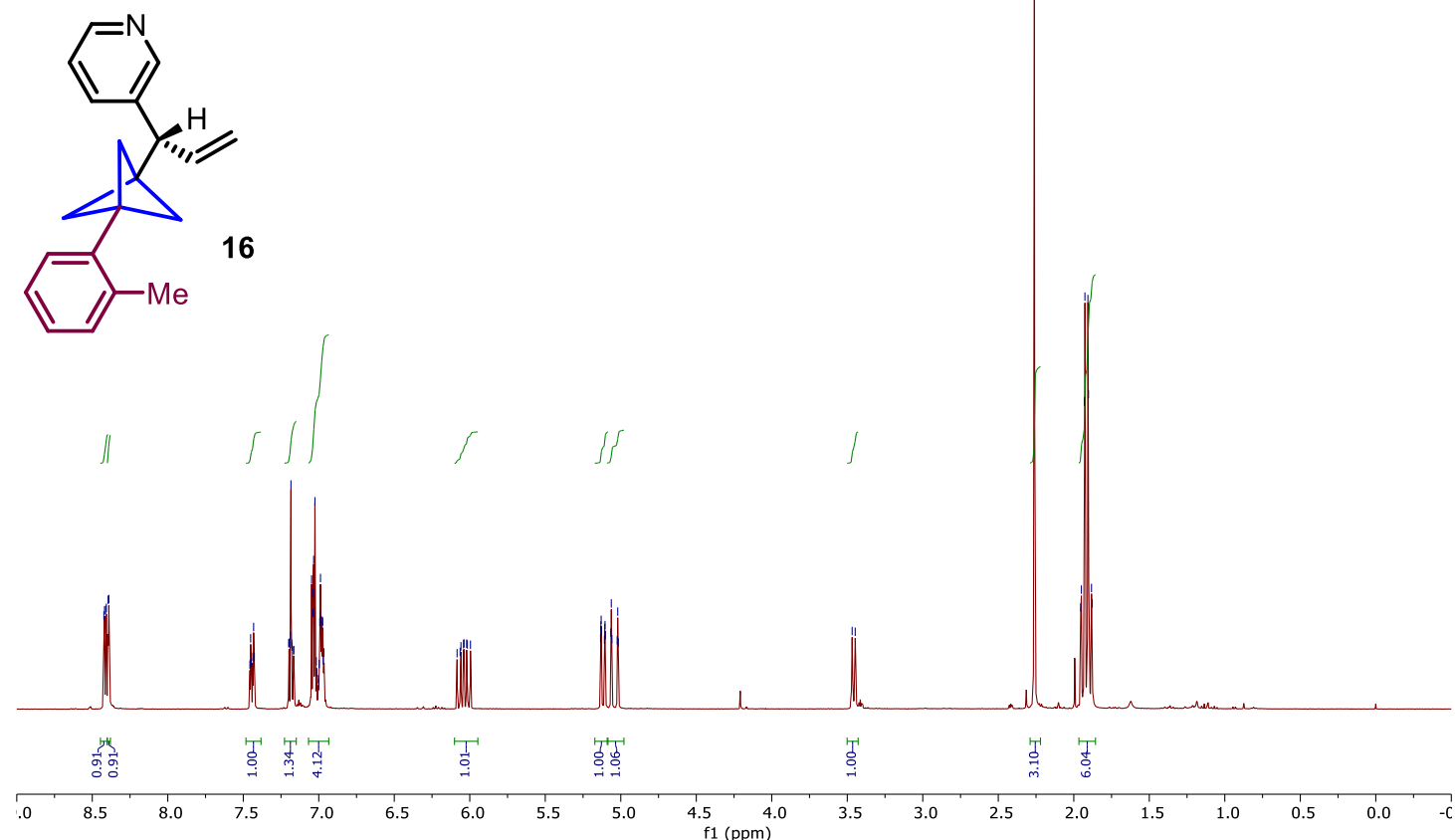

${ }^{13} \mathrm{C} \mathrm{NMR}\left(100 \mathrm{MHz}, \mathrm{CDCl}_{3}\right)$ spectrum for product 16 (see procedure):

58337 yu637.11.fid

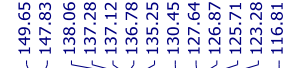

1)
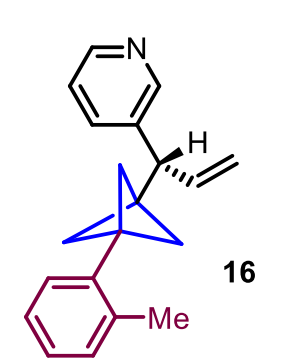

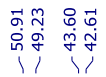

$\infty$
0
0
1
1

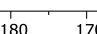

$\quad 160 \quad 150$

$\stackrel{90}{\mathrm{f} 1(\mathrm{ppm})}$ 
${ }^{1} \mathrm{H}$ NMR (400 MHz, $\mathrm{CDCl}_{3}$ ) spectrum for product 17 (see procedure):

58169 yu636.10.fid

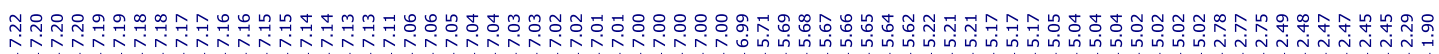
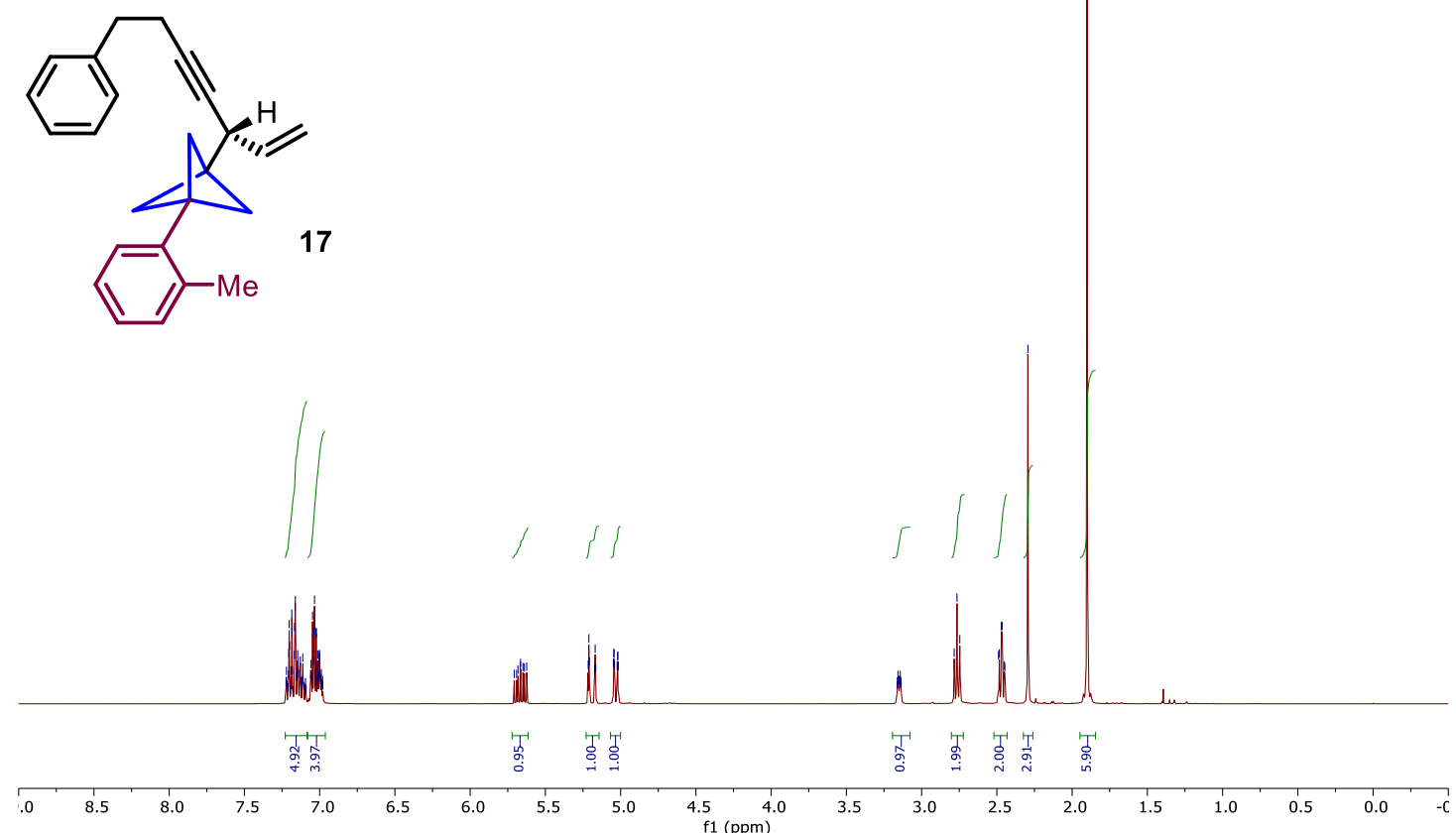

${ }^{13} \mathrm{C}$ NMR (100 MHz, $\mathrm{CDCl}_{3}$ ) spectrum for product 17 (see procedure):
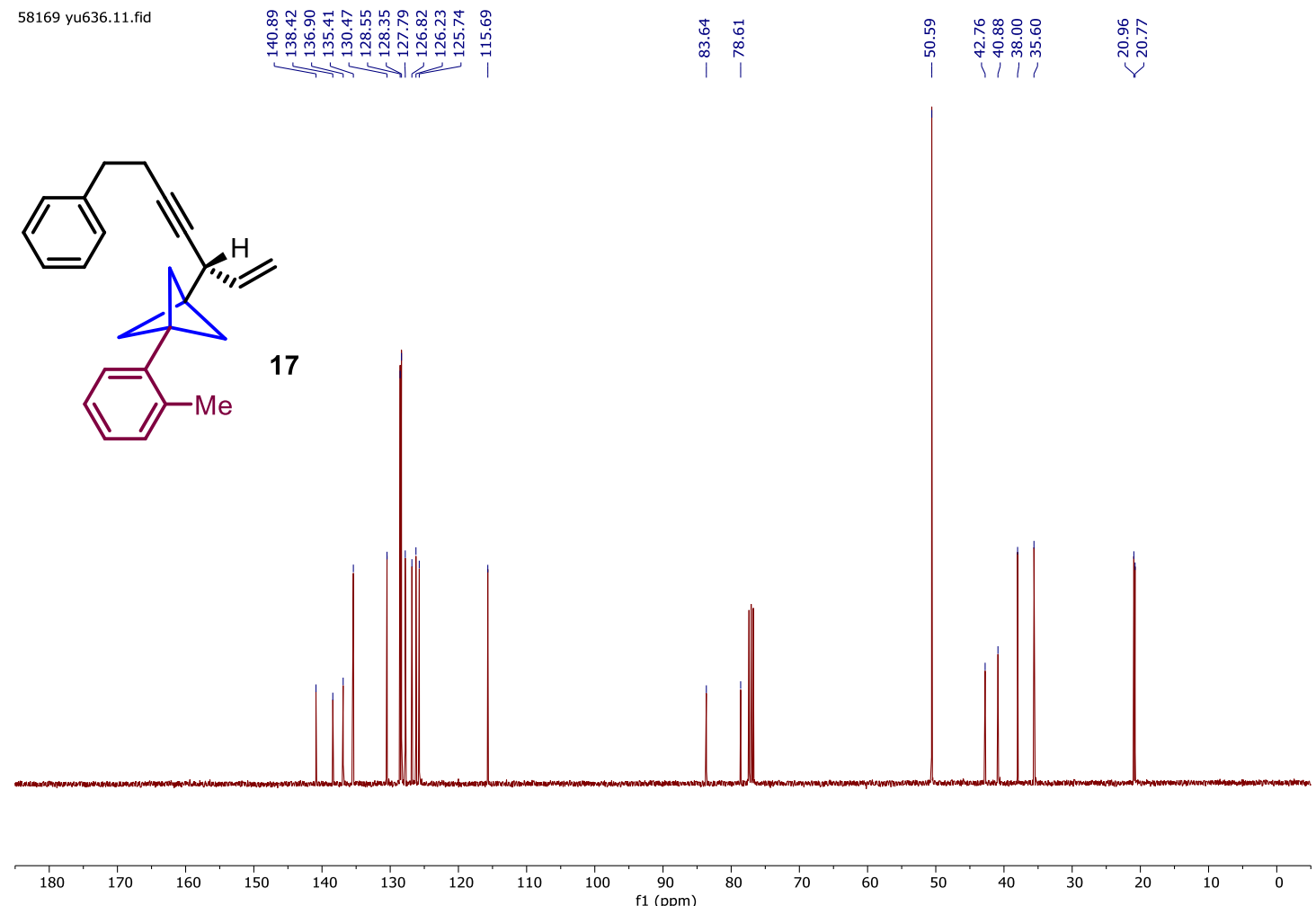
${ }^{1} \mathrm{H}$ NMR (400 MHz, $\mathrm{CDCl}_{3}$ ) spectrum for product $(S, S)-\mathbf{1 8}$ (see procedure):

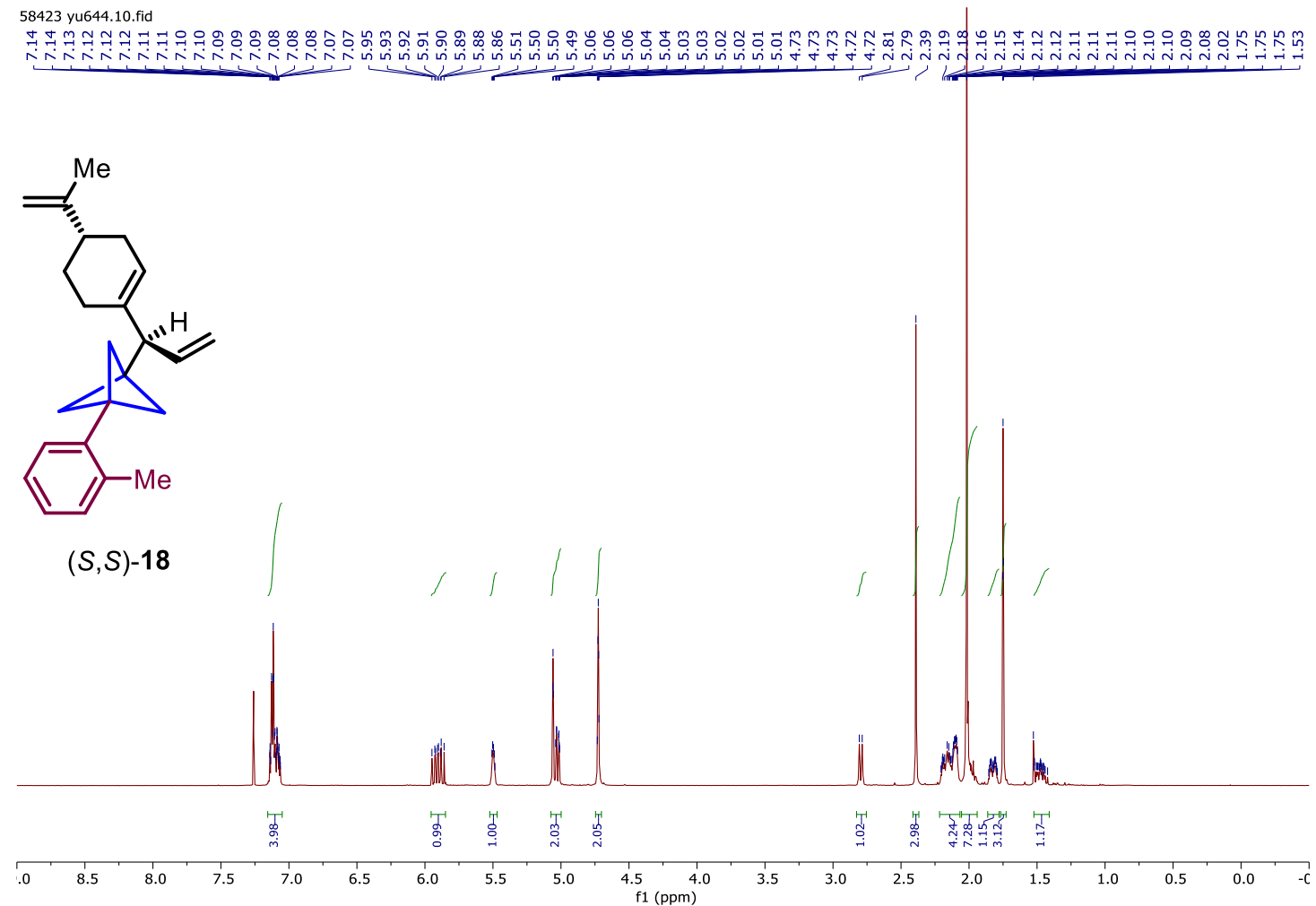

${ }^{13} \mathrm{C}$ NMR (100 MHz, $\left.\mathrm{CDCl}_{3}\right)$ spectrum for product $(S, S)-\mathbf{1 8}$ (see procedure):

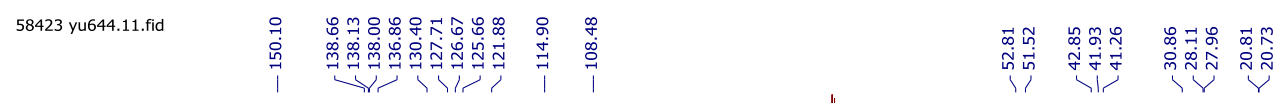

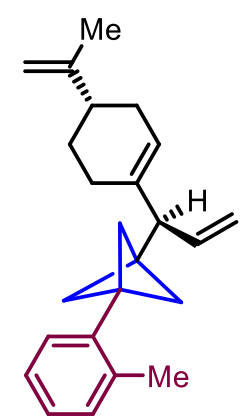

$(S, S)-18$

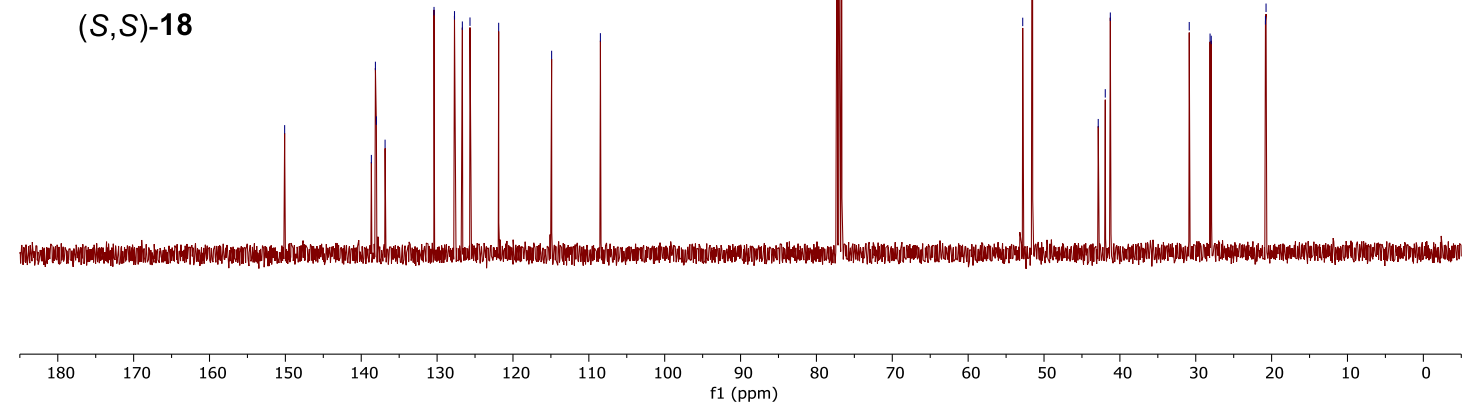


${ }^{1} \mathrm{H}$ NMR (400 MHz, $\left.\mathrm{CDCl}_{3}\right)$ spectrum for product $(R, S)-\mathbf{1 8}$ (see procedure):

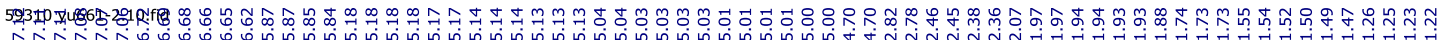

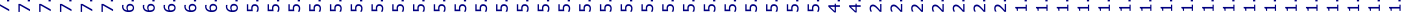

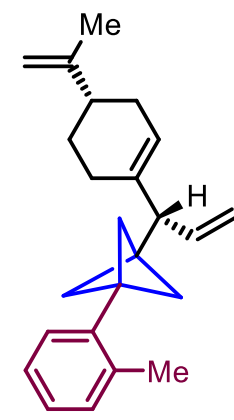

$(R, S)-18$

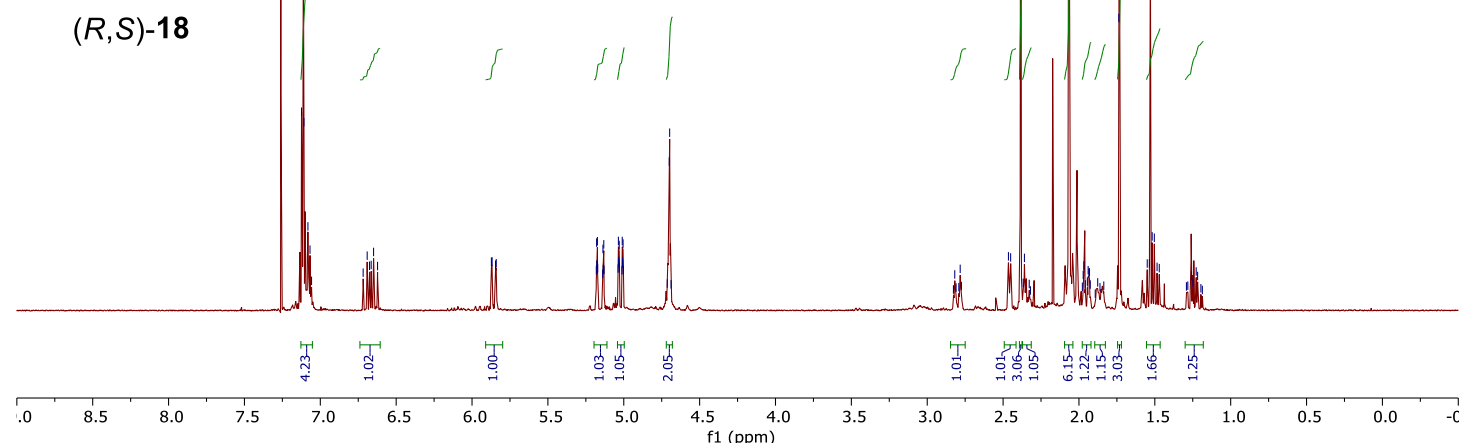

${ }^{13} \mathrm{C} \mathrm{NMR}\left(100 \mathrm{MHz}, \mathrm{CDCl}_{3}\right)$ spectrum for product $(R, S)-\mathbf{1 8}$ (see procedure):

59310 yu661-2.11.fid

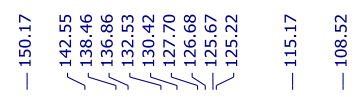

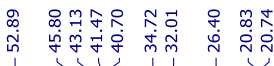

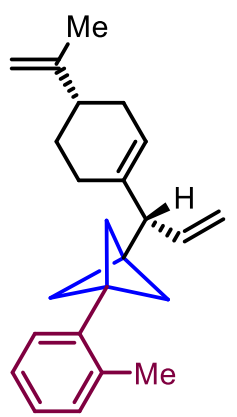

$(R, S)-18$

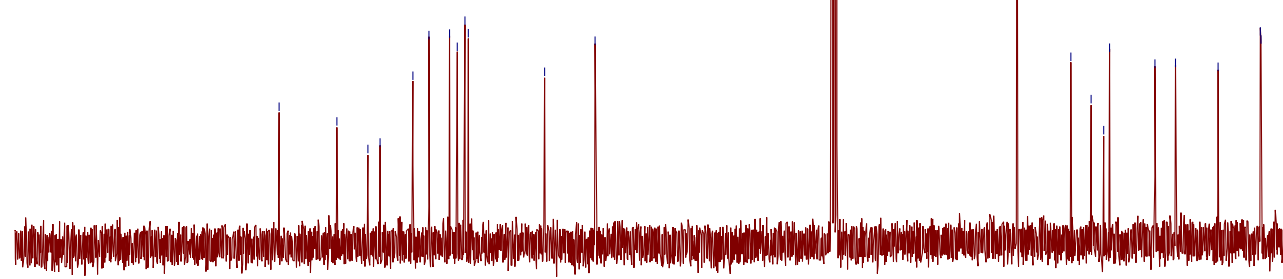

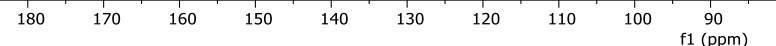


${ }^{1} \mathrm{H}$ NMR (400 MHz, $\mathrm{CDCl}_{3}$ ) spectrum for product 19 (see procedure):

59042 CJ-04-131P.10.fid

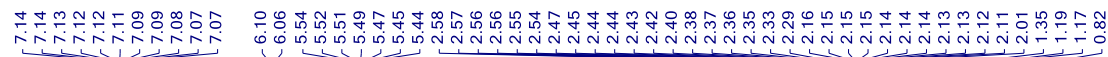

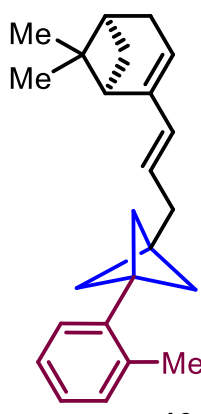

19

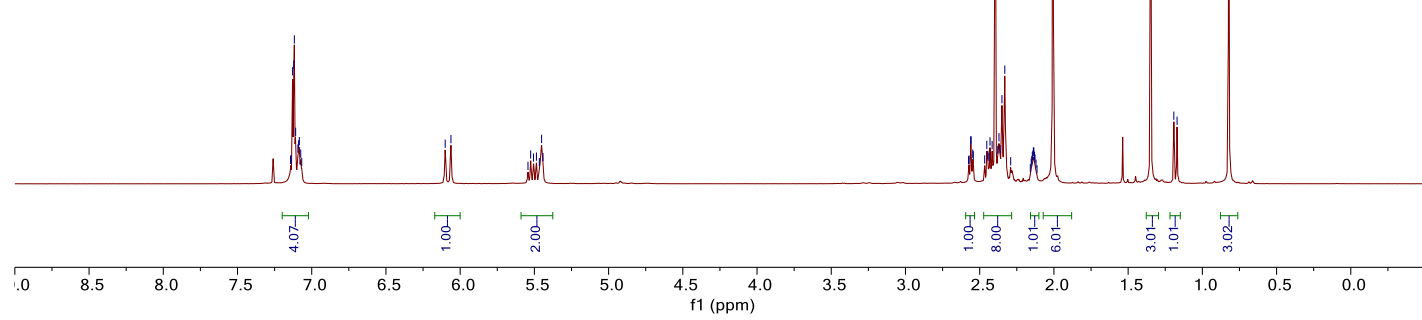

${ }^{13} \mathrm{C}$ NMR (100 MHz, $\mathrm{CDCl}_{3}$ ) spectrum for product 19 (see procedure):

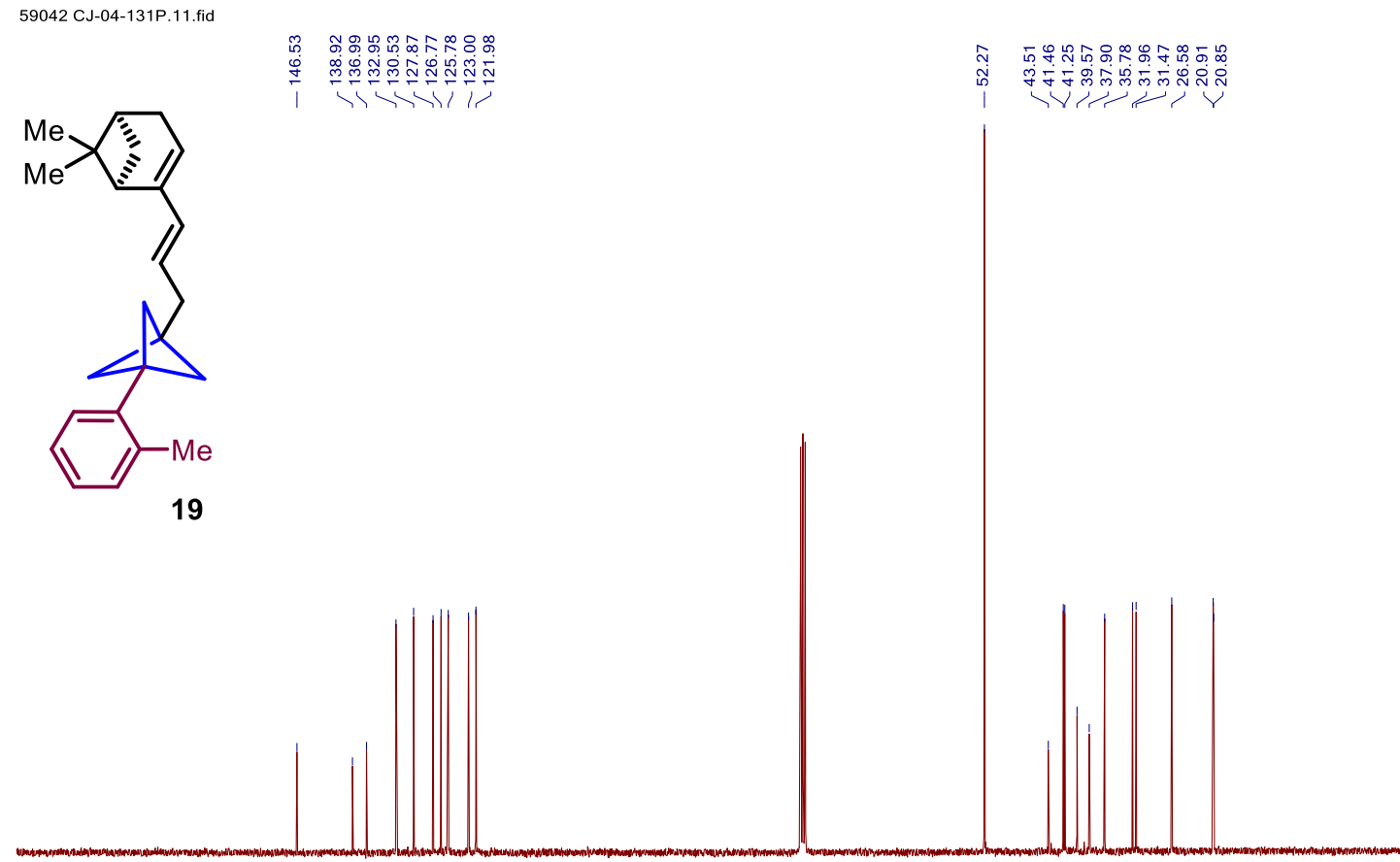

$\begin{array}{lllllllll}180 & 170 & 160 & 150 & 140 & 130 & 120 & 110 & 1\end{array}$ f1 $(\mathrm{ppm})$ 
${ }^{1} \mathrm{H}$ NMR (400 MHz, $\mathrm{CDCl}_{3}$ ) spectrum for product 20 (see procedure):

60192 CJ-04-141P.1.fid

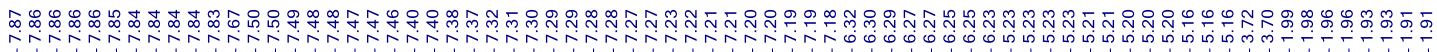

$1=$

${ }_{20}$
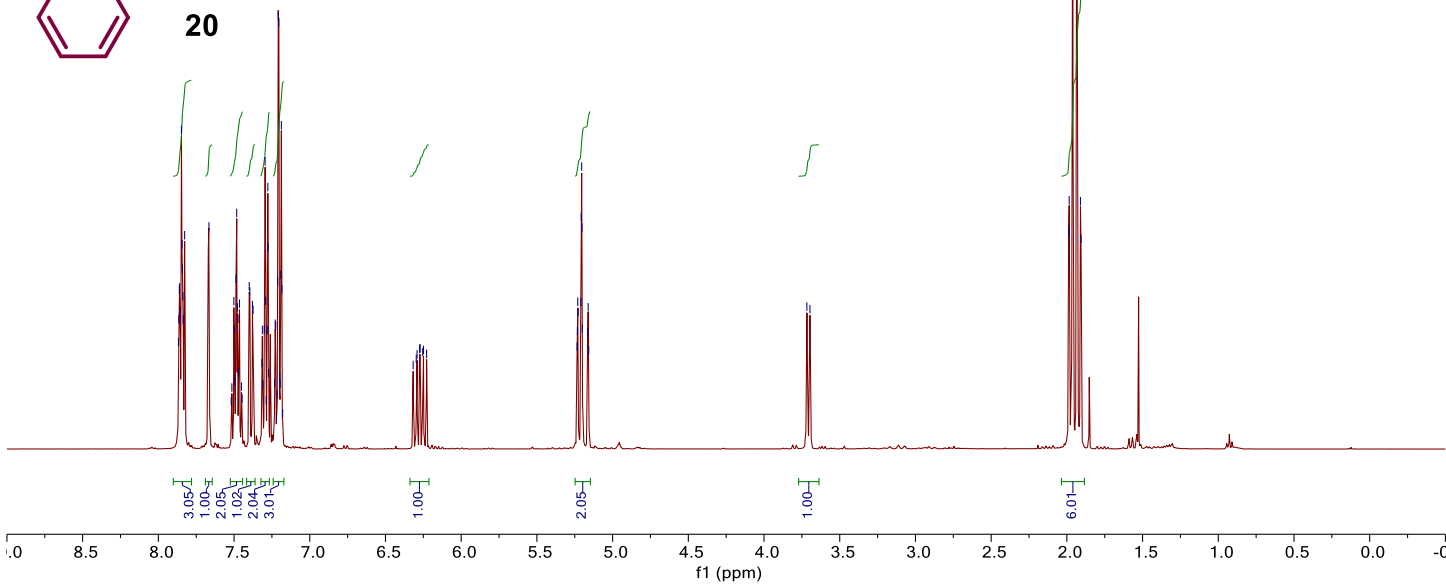

${ }^{13} \mathrm{C}$ NMR (100 $\mathrm{MHz}, \mathrm{CDCl}_{3}$ ) spectrum for product 20 (see procedure):

60192 CJ-04-141P.10.fid

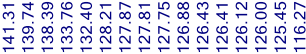
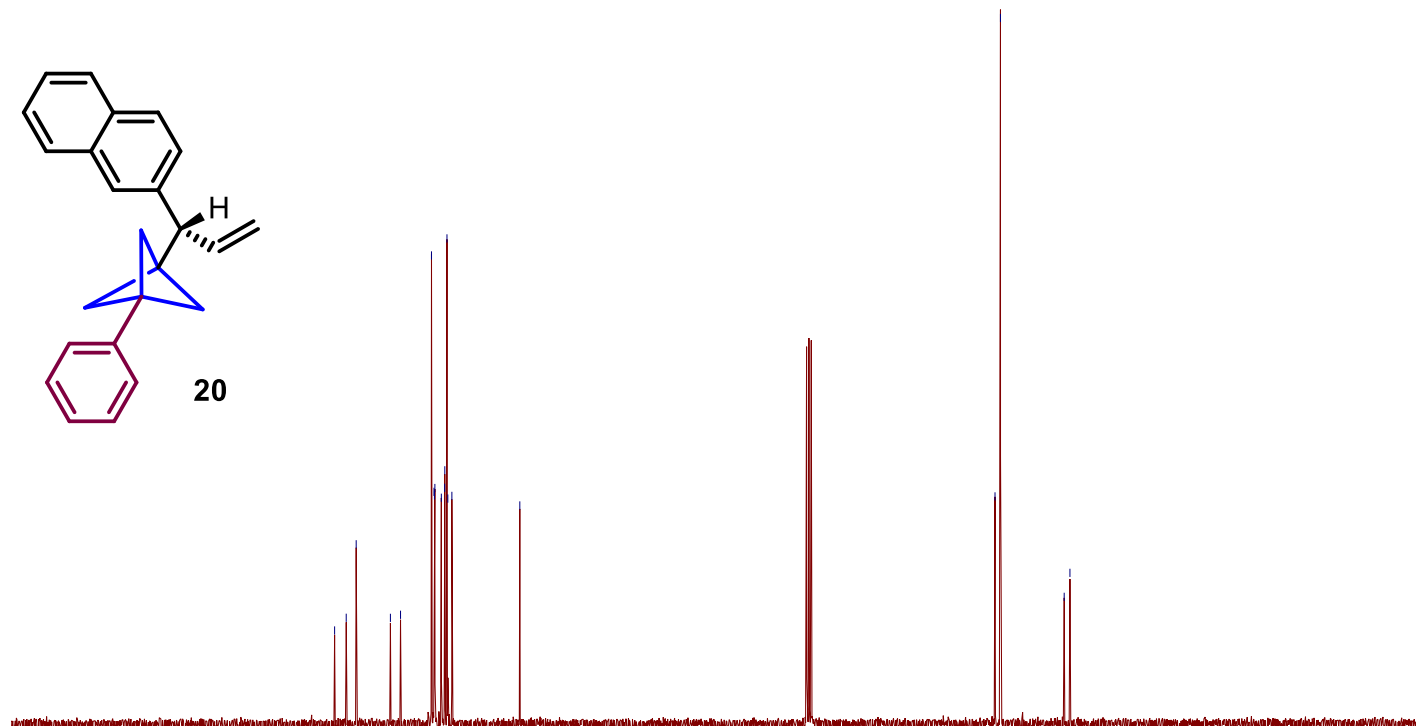

180

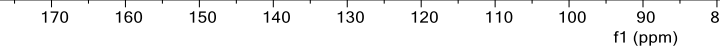

$80 \quad 70 \quad 60$

50

$40 \quad 30 \quad 20$

$10 \quad 0$ 
${ }^{1} \mathrm{H}$ NMR (400 MHz, $\mathrm{CDCl}_{3}$ ) spectrum for product 21 (see procedure):

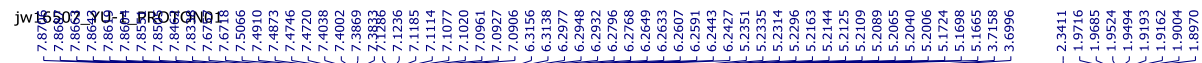

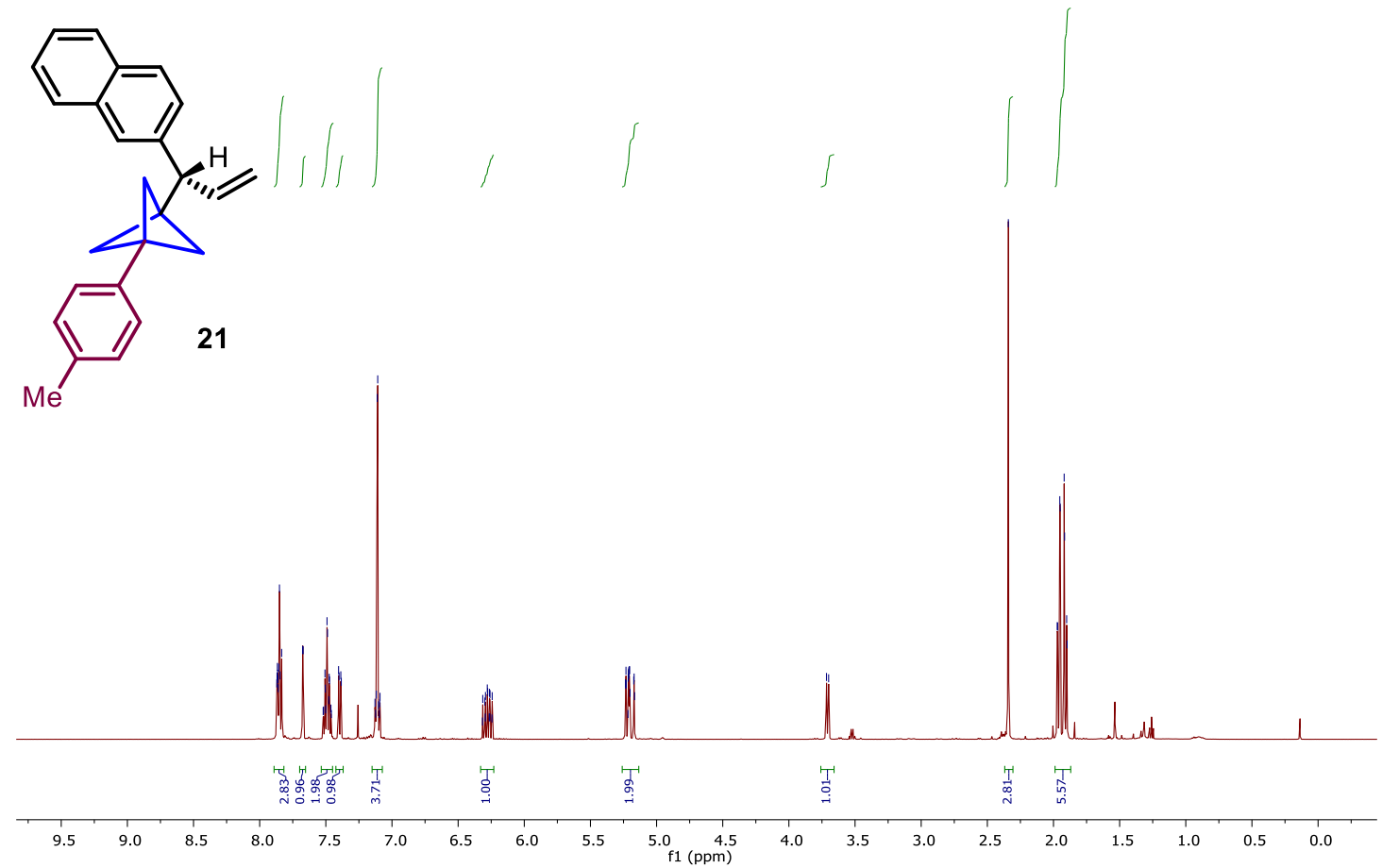

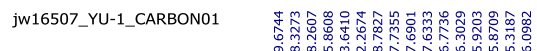

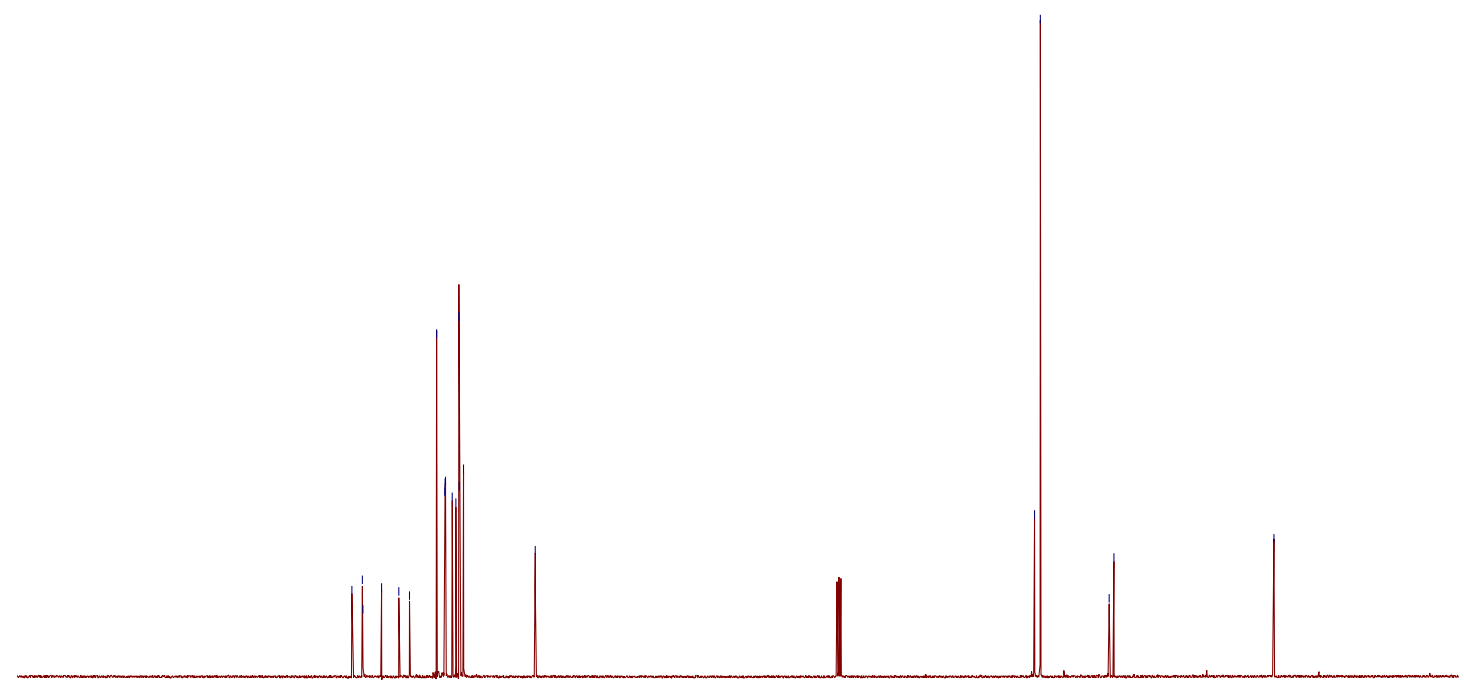

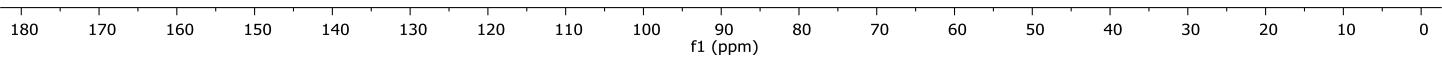


${ }^{1} \mathrm{H}$ NMR (400 MHz, $\mathrm{CDCl}_{3}$ ) spectrum for product 22 (see procedure):

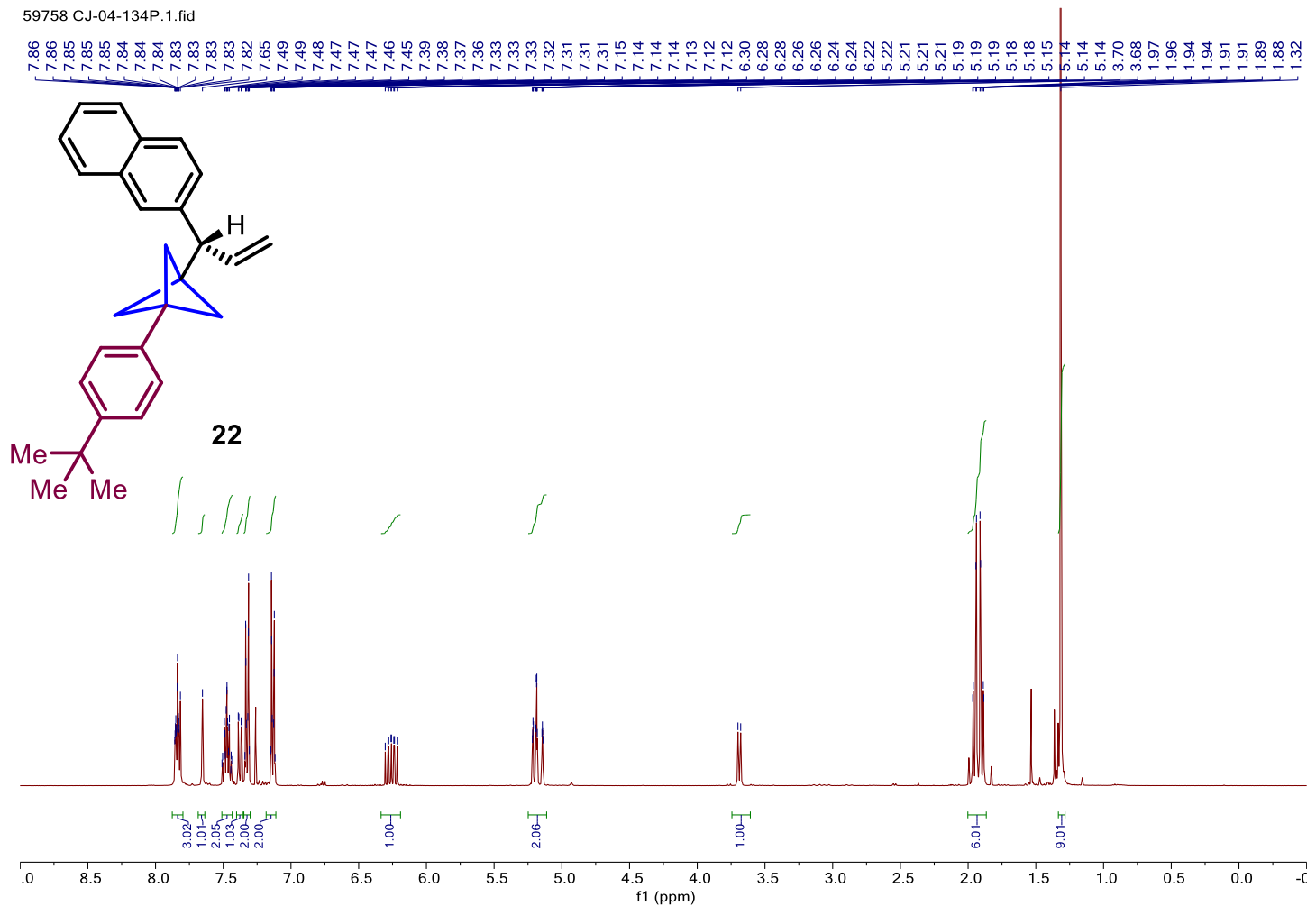

${ }^{13} \mathrm{C} \mathrm{NMR}\left(100 \mathrm{MHz}, \mathrm{CDCl}_{3}\right)$ spectrum for product 22 (see procedure):

59758 CJ-04-134P.11.fid

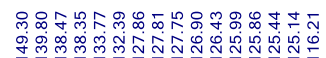

每

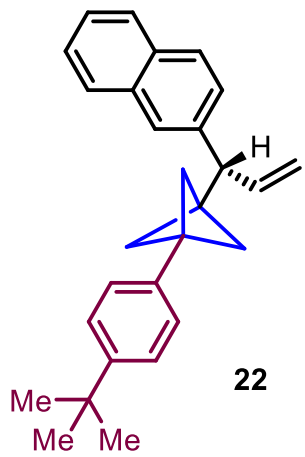

22
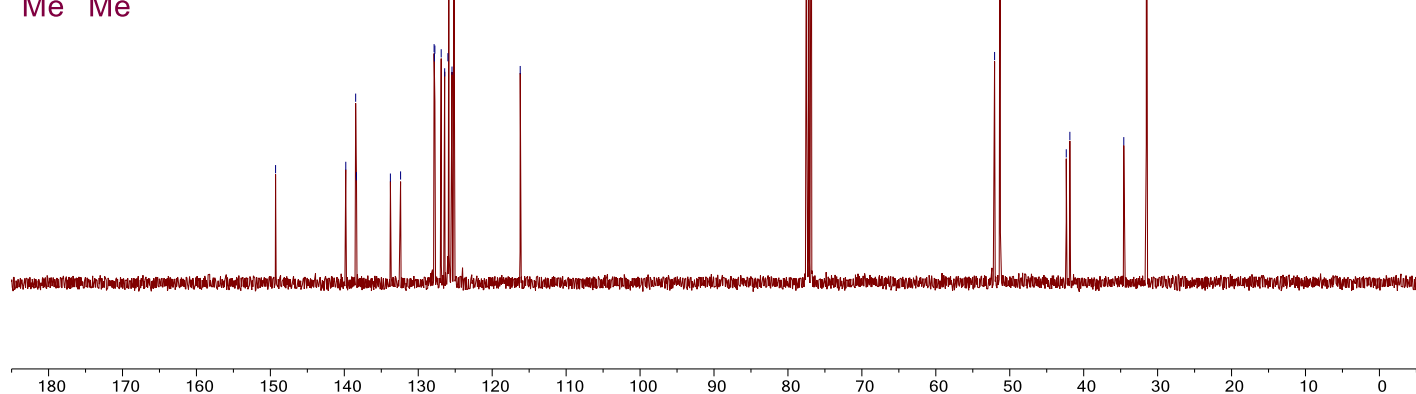

$120 \quad 110 \quad 100$

90
f1 $(\mathrm{ppm})$

$\begin{array}{ll}1 & 1 \\ & 70\end{array}$

50

$10 \quad 0$ 
${ }^{1} \mathrm{H}$ NMR (400 MHz, $\mathrm{CDCl}_{3}$ ) spectrum for product 23a (see procedure):

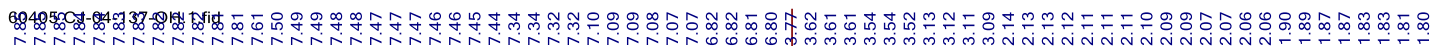

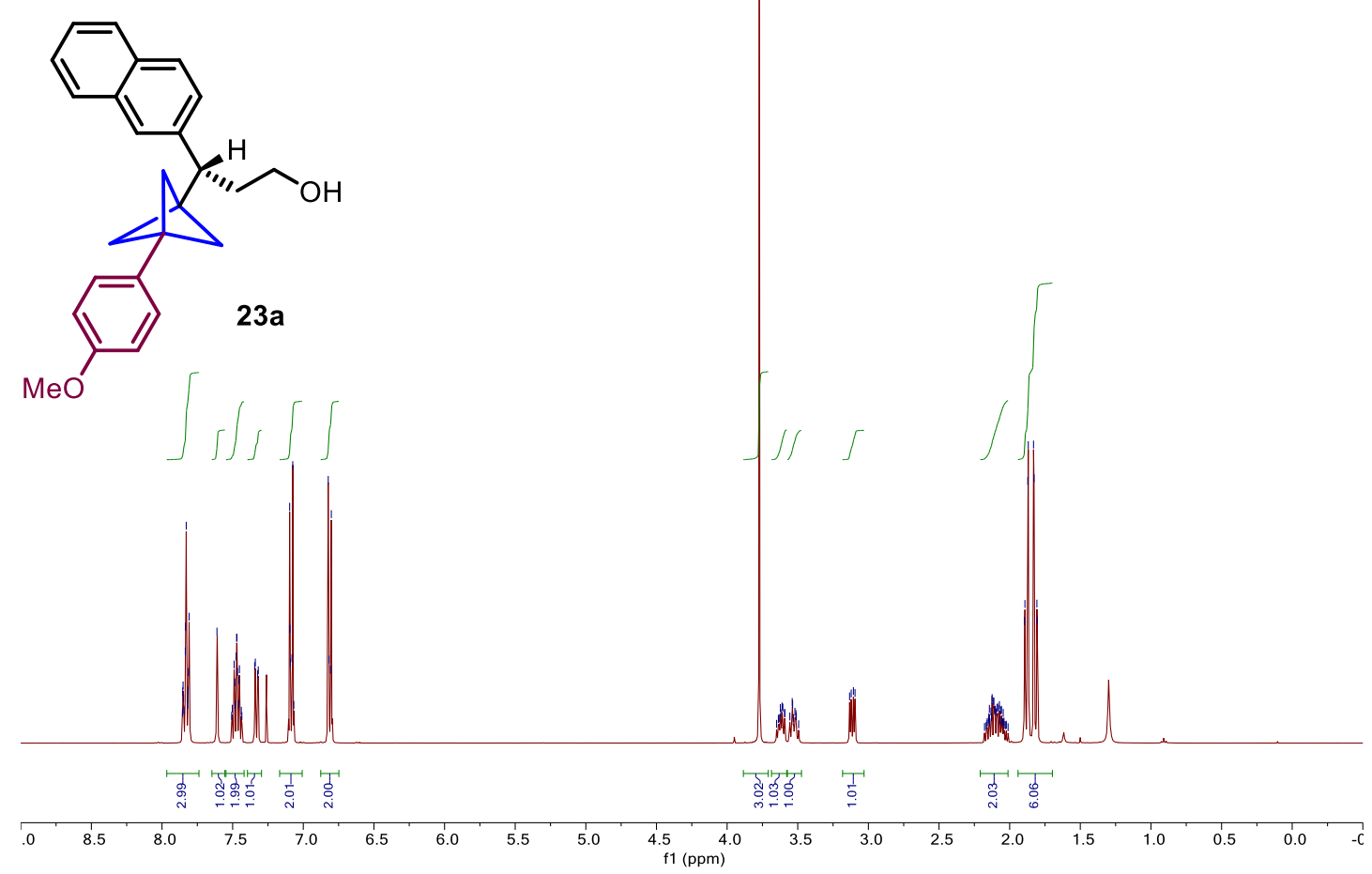

${ }^{13} \mathrm{C}$ NMR (100 MHz, $\mathrm{CDCl}_{3}$ ) spectrum for product 23a (see procedure):

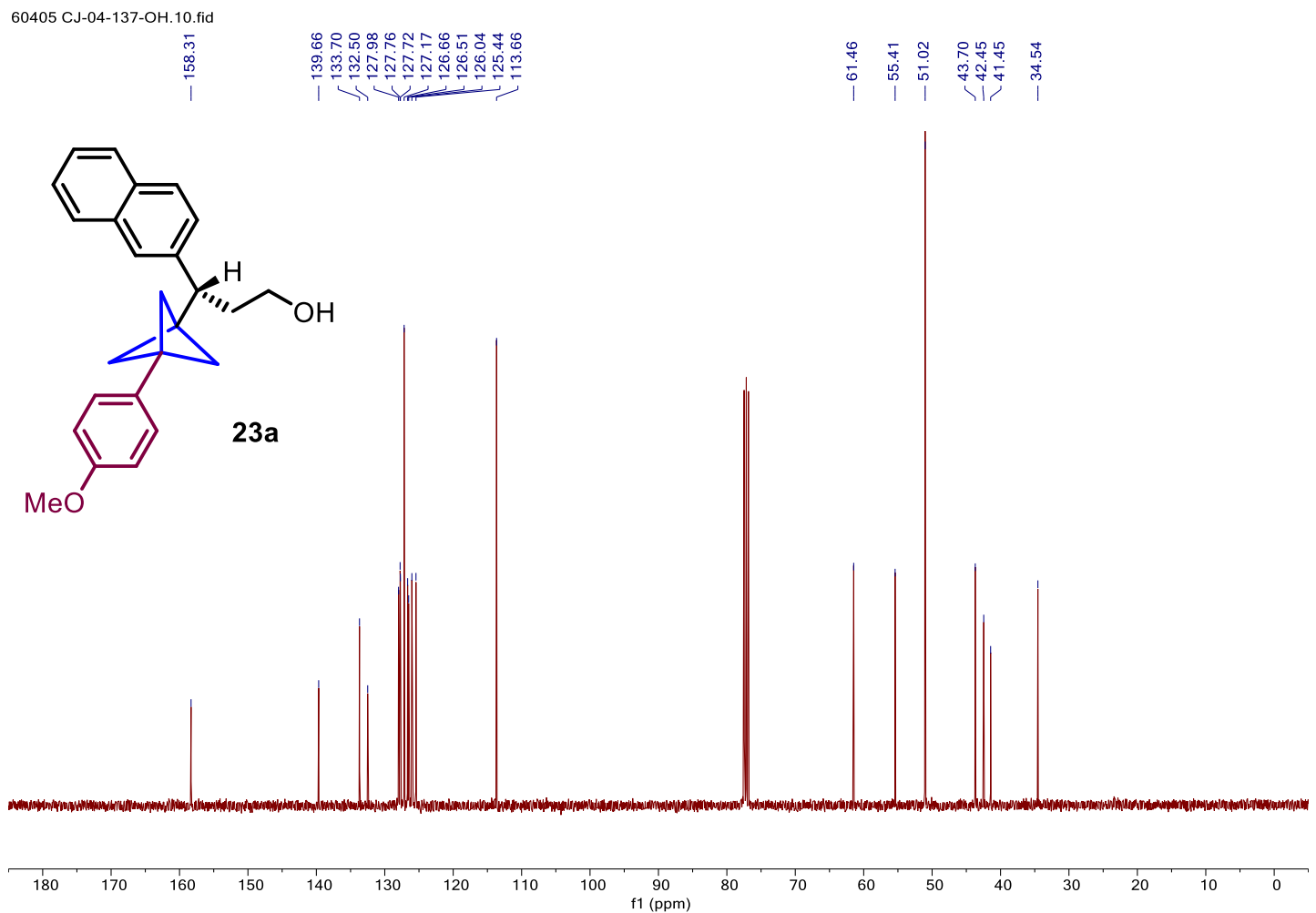


${ }^{1} \mathrm{H}$ NMR (400 MHz, $\mathrm{CDCl}_{3}$ ) spectrum for product 24 (see procedure):

60990 CJ-04-135P.1.fid

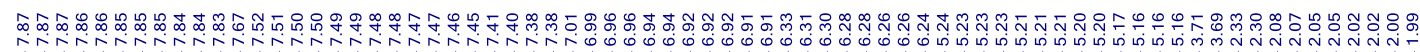

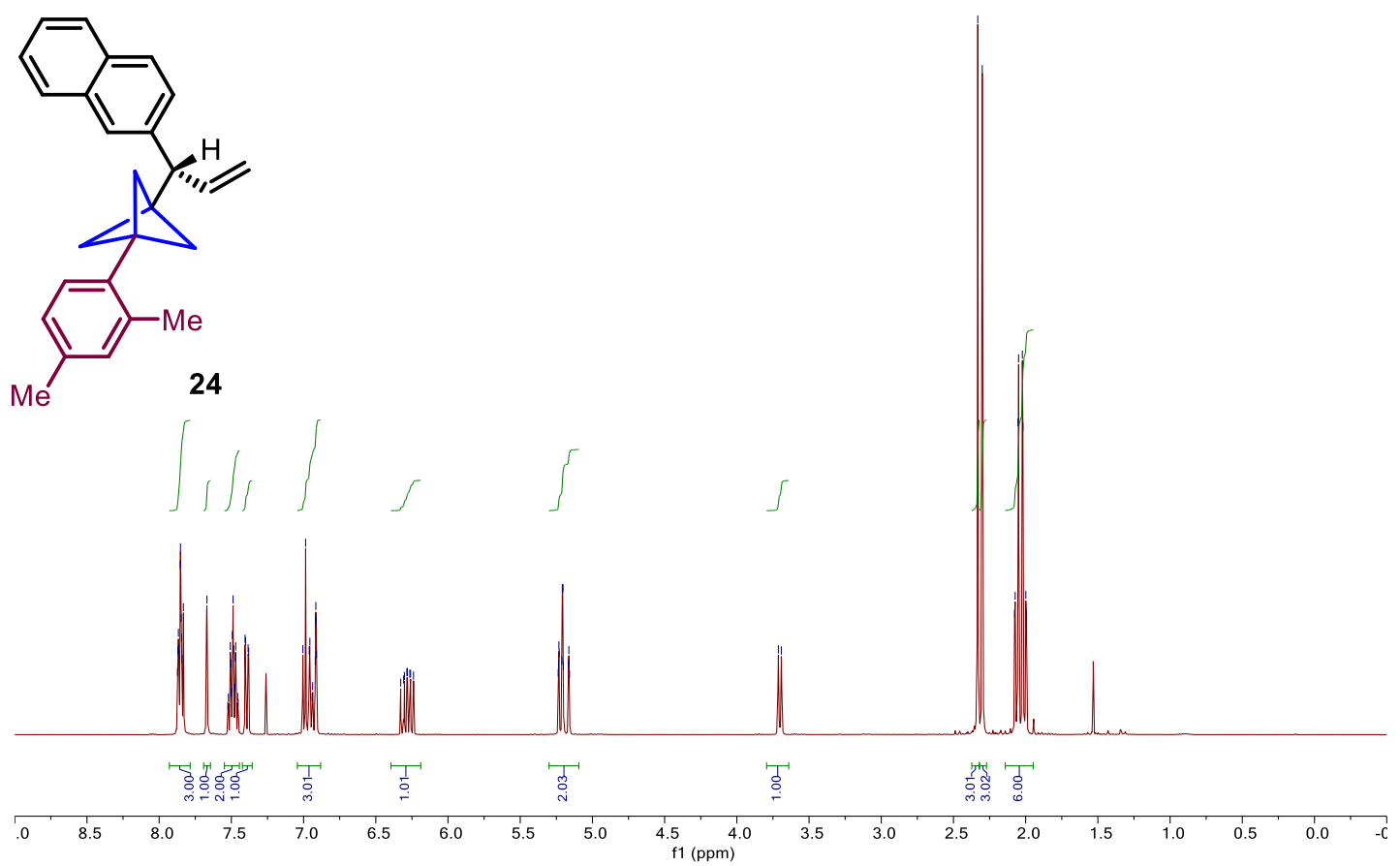

${ }^{13} \mathrm{C} \mathrm{NMR}\left(100 \mathrm{MHz}, \mathrm{CDCl}_{3}\right.$ ) spectrum for product 24 (see procedure):

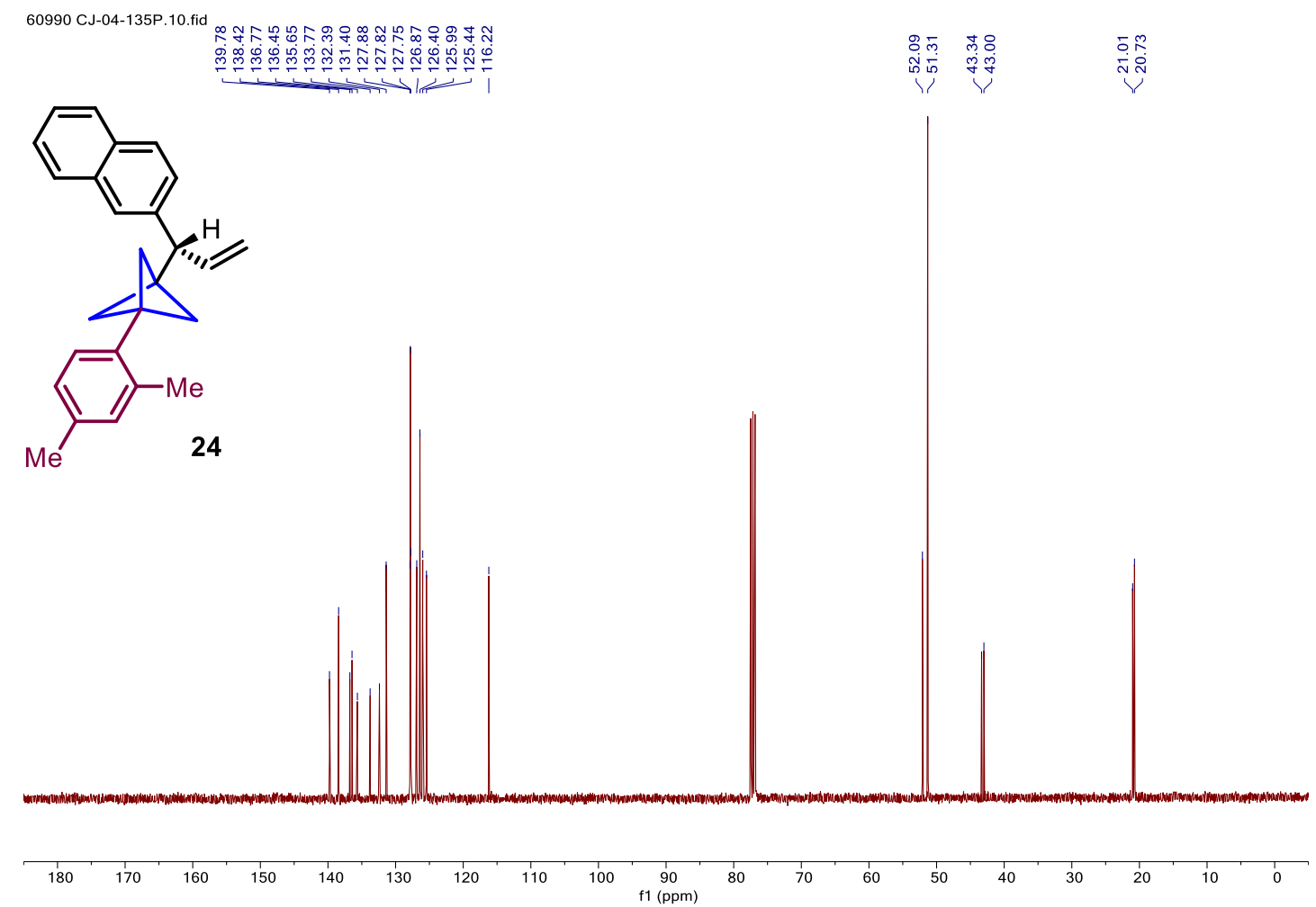


${ }^{1} \mathrm{H}$ NMR (400 MHz, $\mathrm{CDCl}_{3}$ ) spectrum for product 25 (see procedure):

585

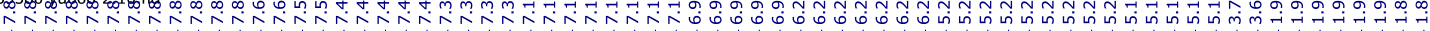

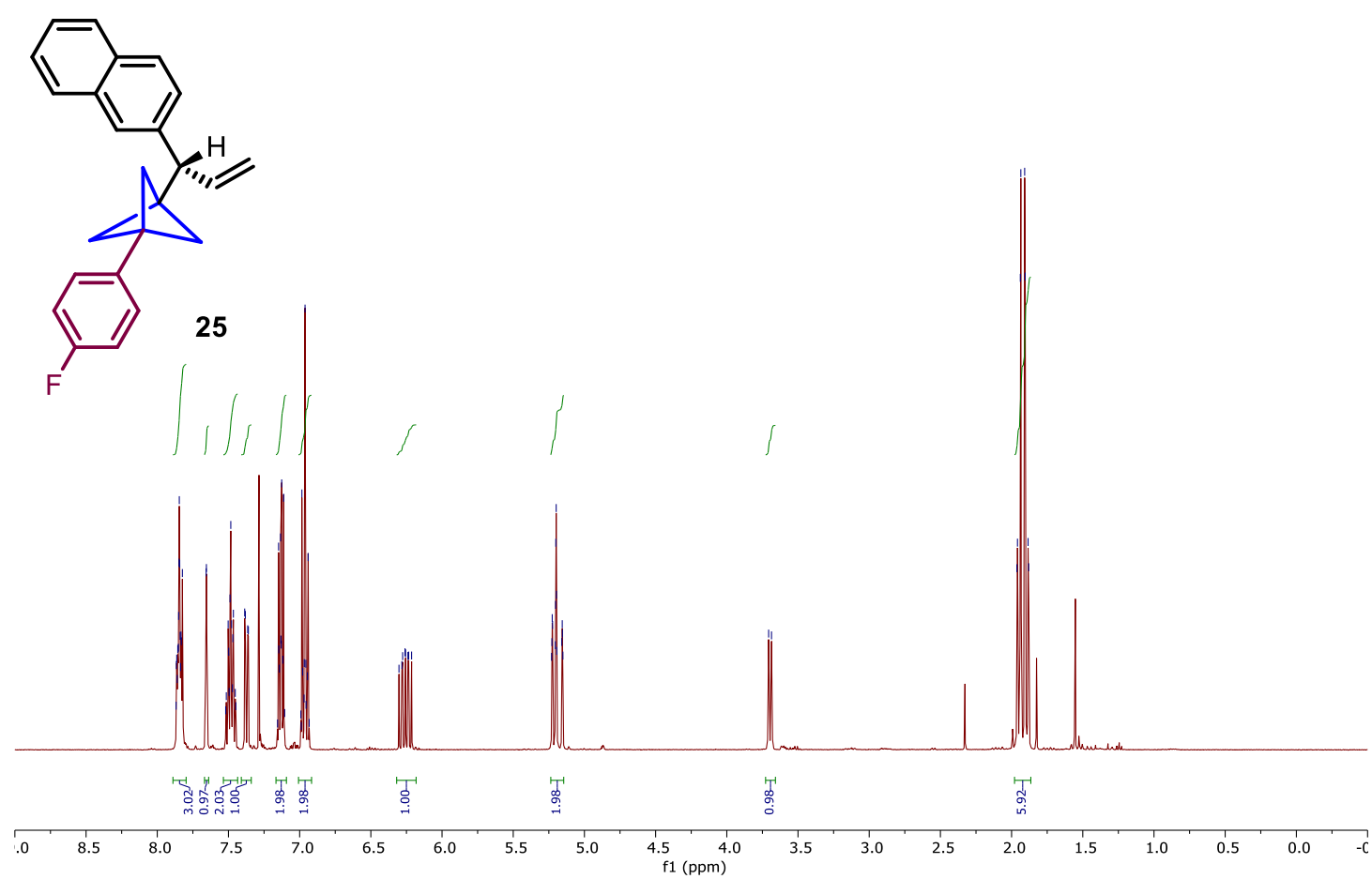

${ }^{13} \mathrm{C}$ NMR (100 MHz, $\mathrm{CDCl}_{3}$ ) spectrum for product 25 (see procedure):

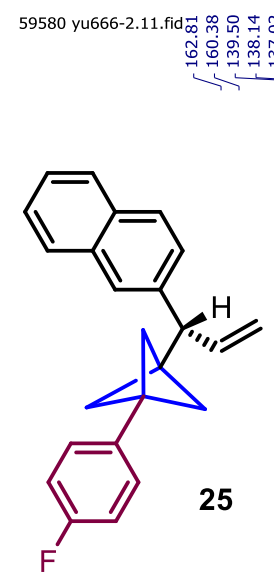

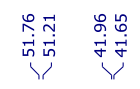

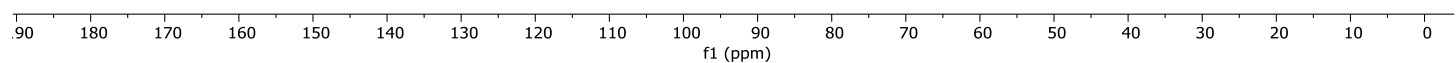


${ }^{1} \mathrm{H}$ NMR (400 MHz, $\mathrm{CDCl}_{3}$ ) spectrum for product 26 (see procedure):

60117 CJ-04-140P.1.fid

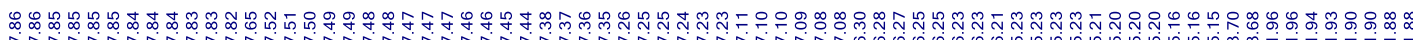

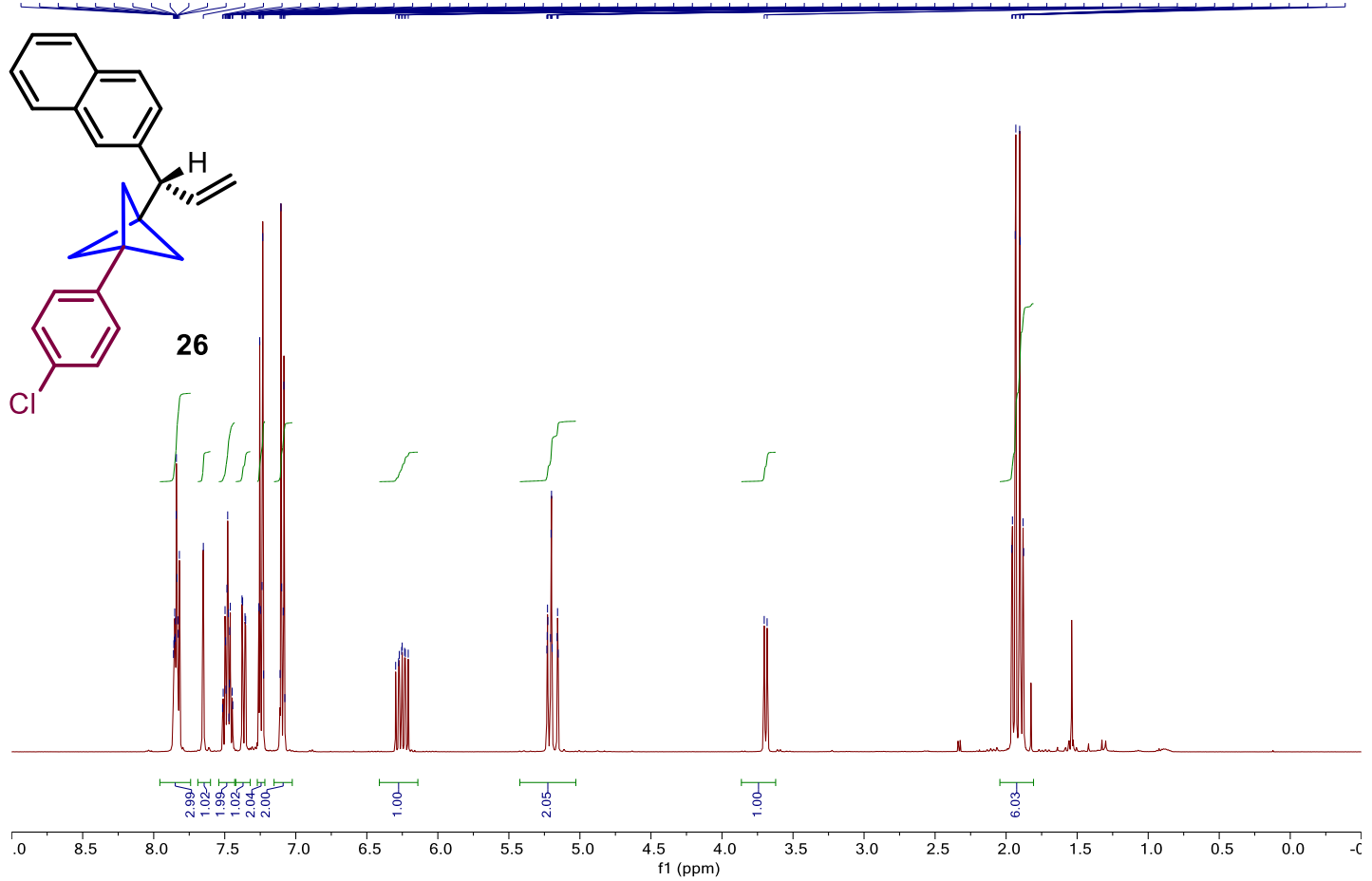

${ }^{13} \mathrm{C}$ NMR (100 MHz, $\mathrm{CDCl}_{3}$ ) spectrum for product 26 (see procedure):

60117 CJ-04-140P.10.fid

D.

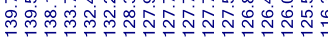

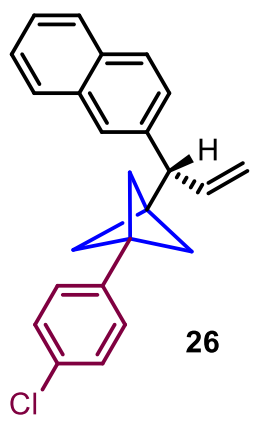

成

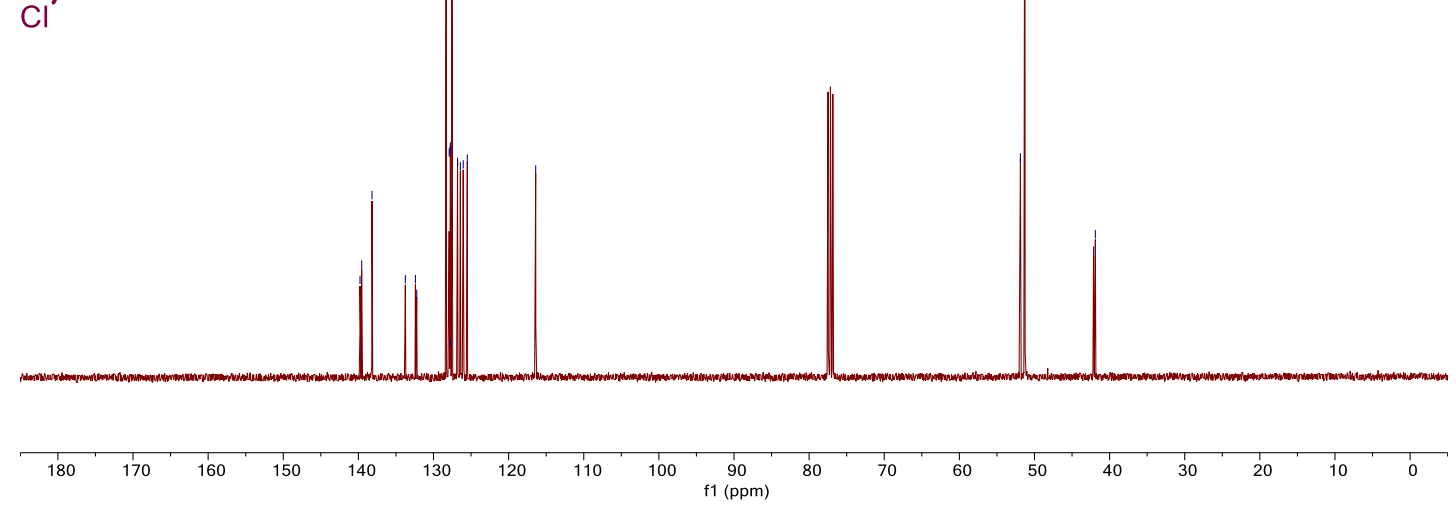


${ }^{1} \mathrm{H}$ NMR (400 MHz, $\mathrm{CDCl}_{3}$ ) spectrum for product 27 (see procedure):

61386 CJ-04-138P.1.fid

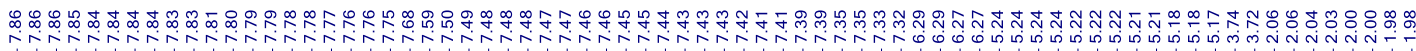

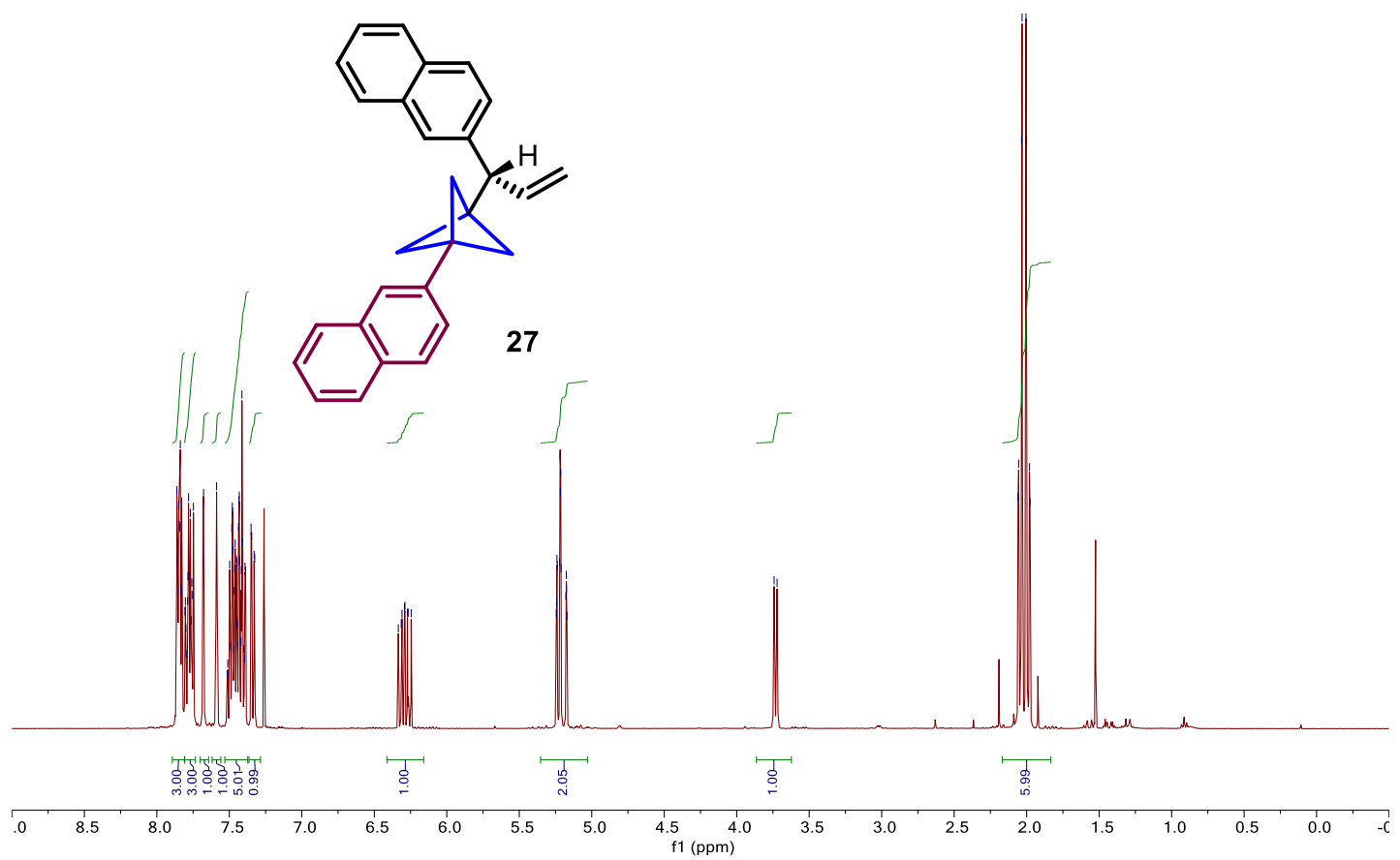

${ }^{13} \mathrm{C} \mathrm{NMR}\left(100 \mathrm{MHz}, \mathrm{CDCl}_{3}\right)$ spectrum for product 27 (see procedure):

59994 CJ-04-138P.10.fid

món

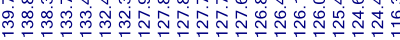

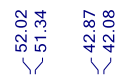
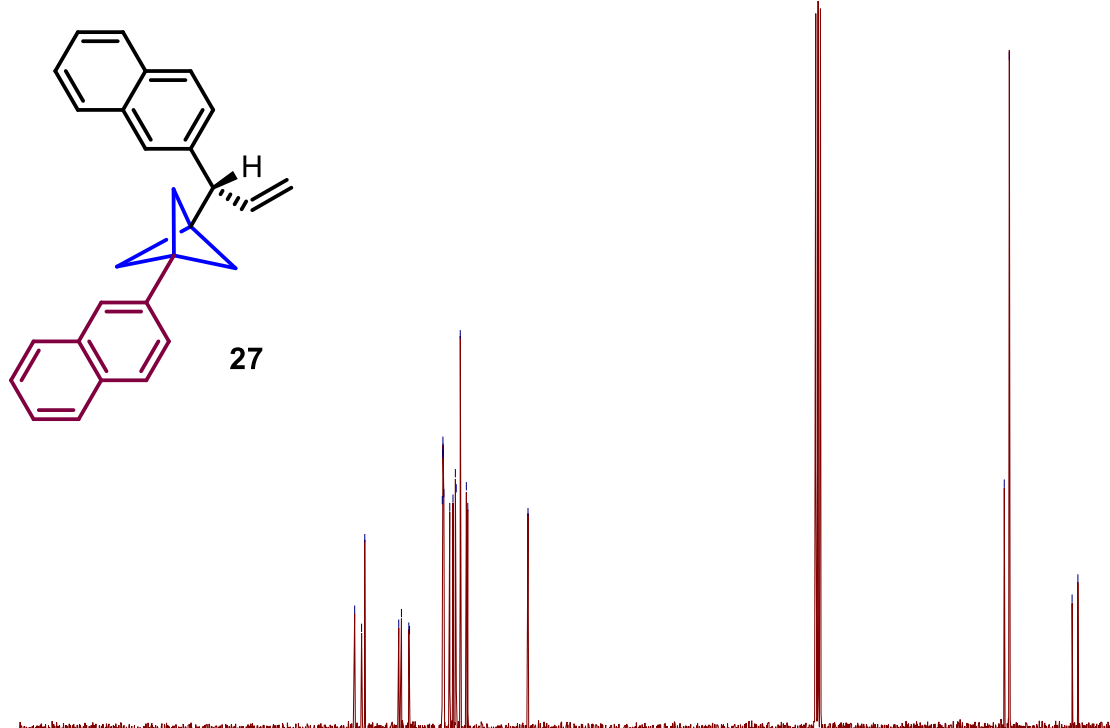

$\begin{array}{lllllllll}180 & 170 & 160 & 150 & 140 & 130 & 120 & 110 & 100\end{array}$ f1 $(\mathrm{ppm})$

$80 \quad 70$

$60 \quad 50$

$\begin{array}{llllll}1 & 1 & 1 & 1 & 1 & 1 \\ 40 & 20 & 10 & 0\end{array}$ 
${ }^{1} \mathrm{H}$ NMR (400 MHz, $\mathrm{CDCl}_{3}$ ) spectrum for product 28 (see procedure):

61557 CJ-04-147P.1.fid

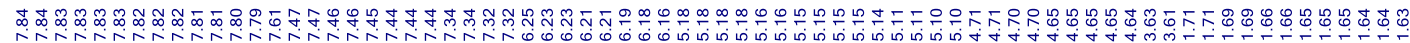
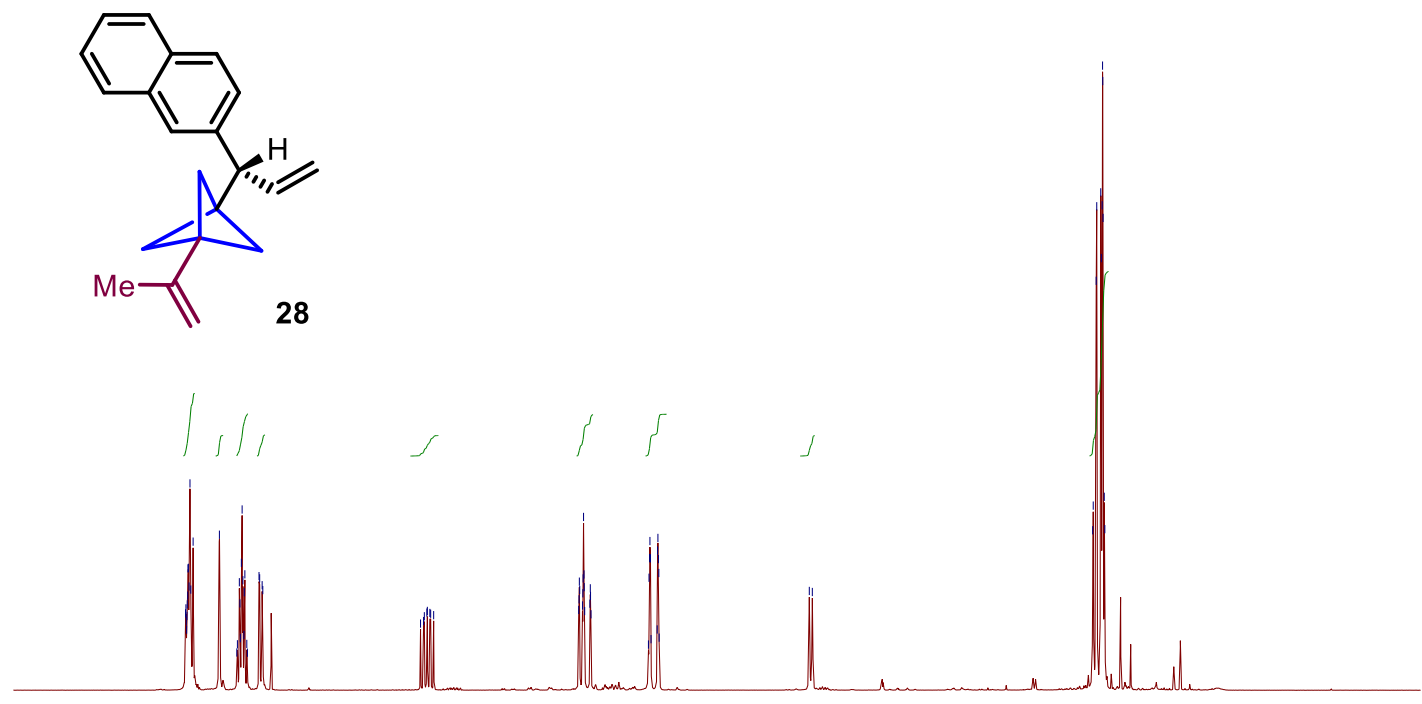

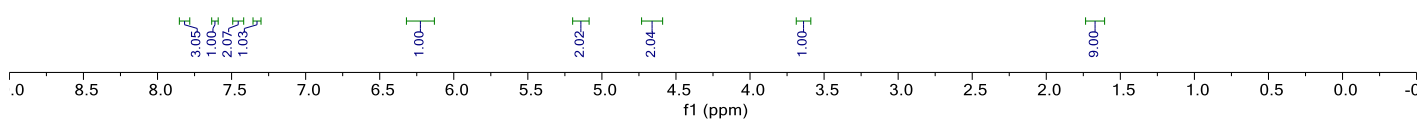

${ }^{13} \mathrm{C}$ NMR (100 MHz, $\mathrm{CDCl}_{3}$ ) spectrum for product 28 (see procedure):
61557 CJ-04-147P.10.fic

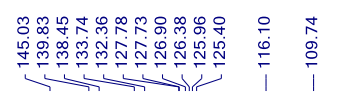

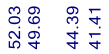
$\stackrel{8}{\stackrel{\circ}{\circ}}$
1

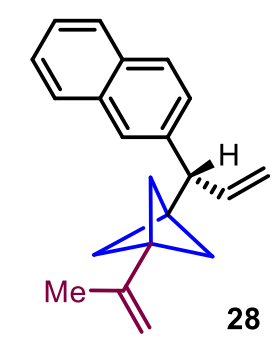

28

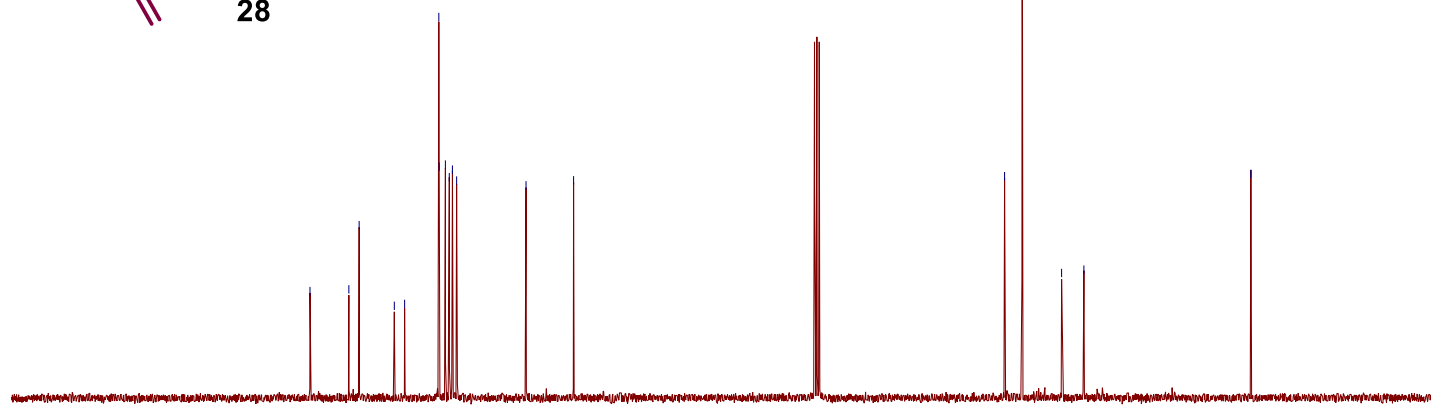

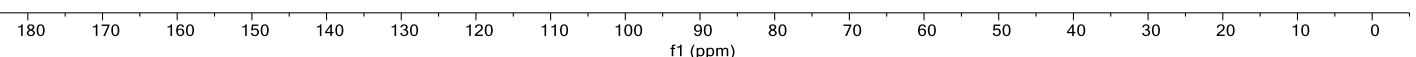


${ }^{1} \mathrm{H}$ NMR (400 MHz, $\mathrm{CDCl}_{3}$ ) spectrum for product 29 (see procedure):

\section{2 yu667.10.fid}

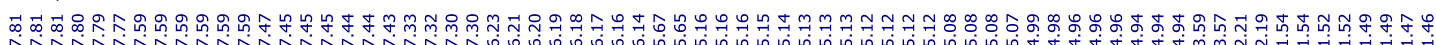

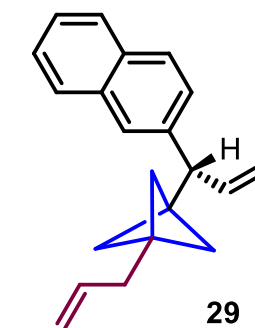

29
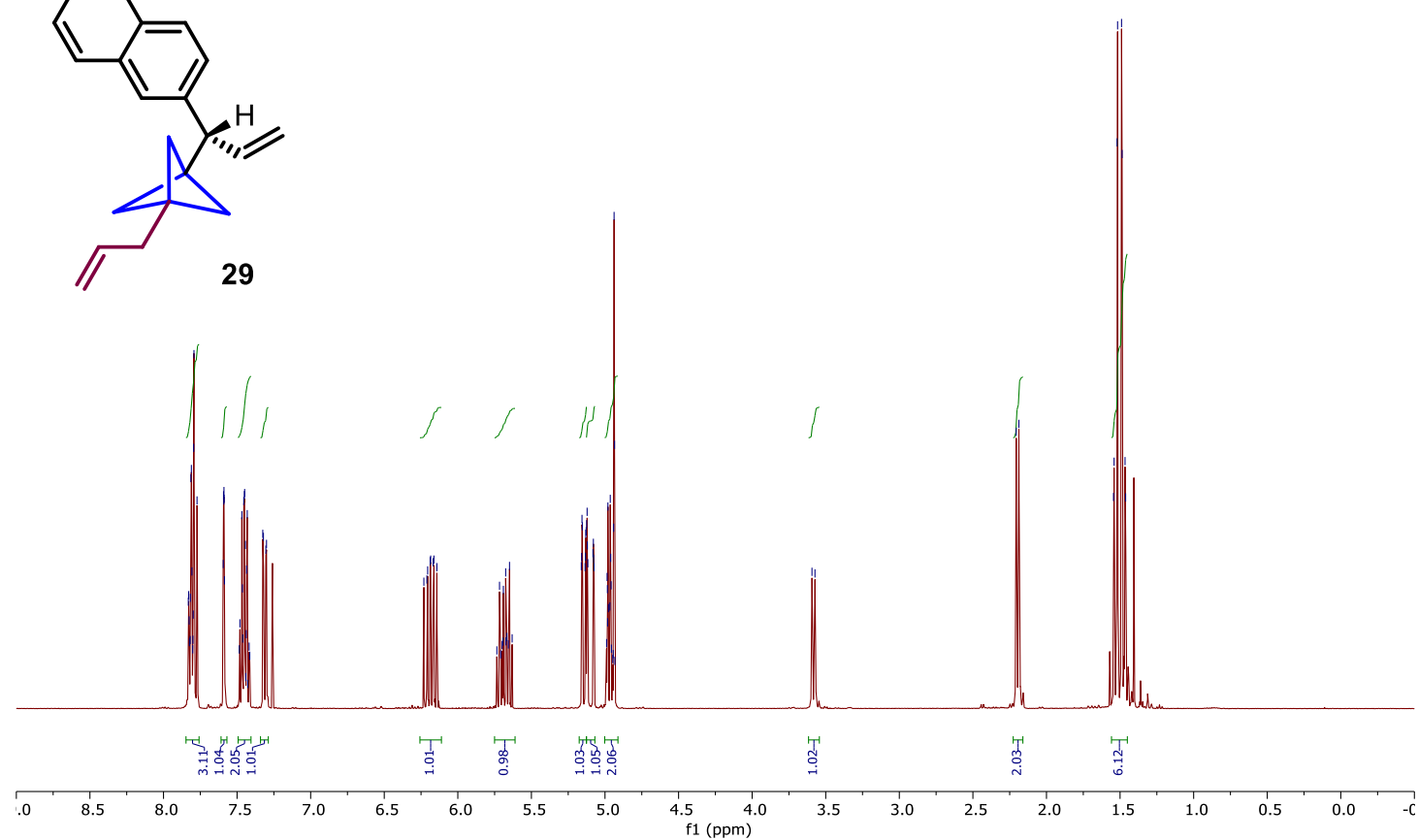

${ }^{13} \mathrm{C}$ NMR (100 MHz, $\mathrm{CDCl}_{3}$ ) spectrum for product 29 (see procedure):

59772 yu667.11.fid

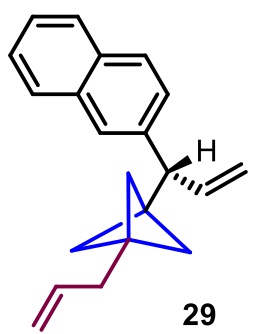

29

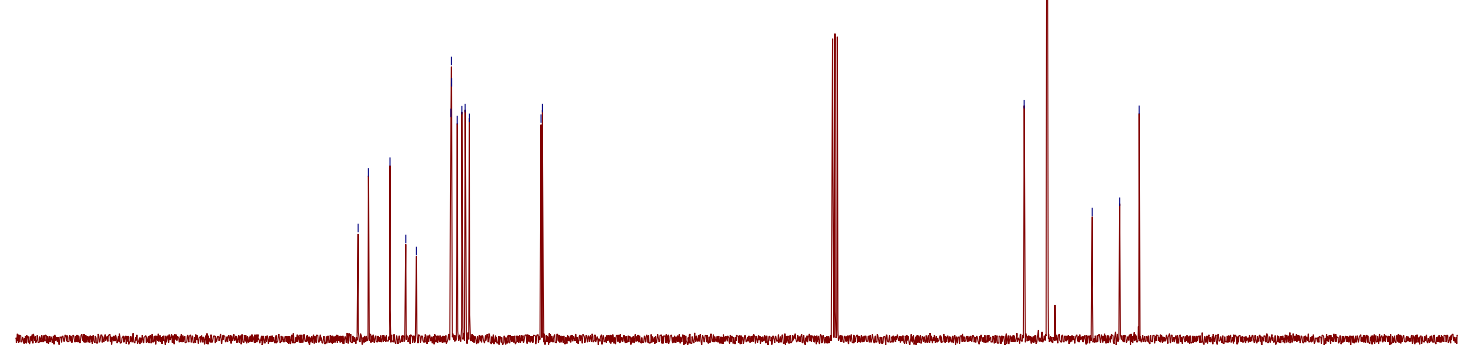

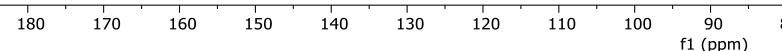


${ }^{1} \mathrm{H}$ NMR (400 MHz, $\mathrm{CDCl}_{3}$ ) spectrum for product 30a (see procedure):

61130 yu706 oh.10.fid

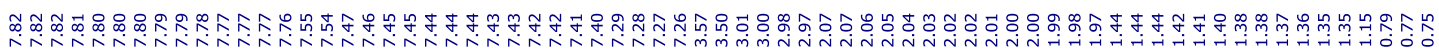<smiles>CCC12CCC(CCO)(CC1c1ccc3ccccc3c1)C2</smiles>
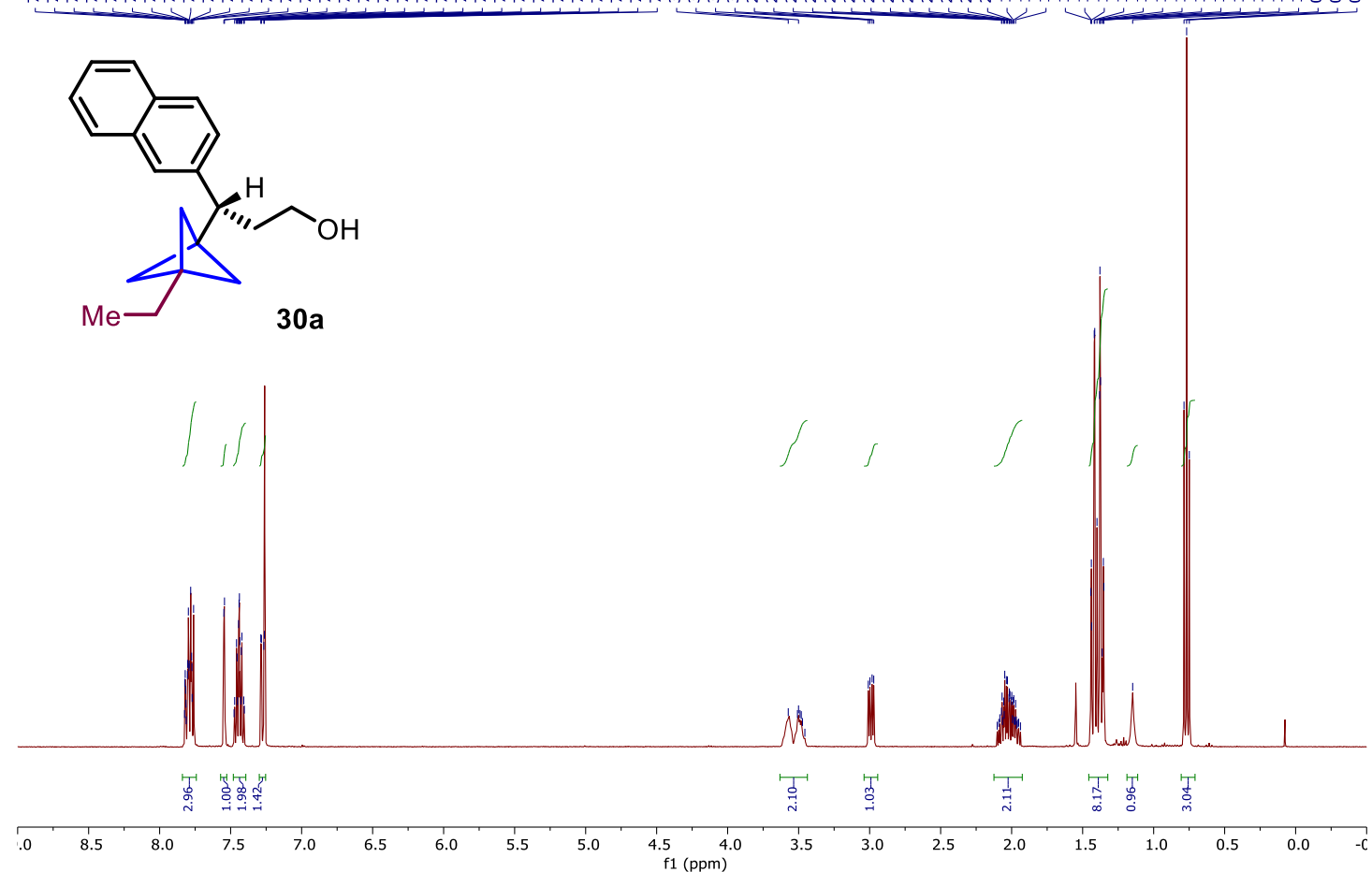

${ }^{13} \mathrm{C}$ NMR (100 MHz, $\left.\mathrm{CDCl}_{3}\right)$ spectrum for product 30a (see procedure):

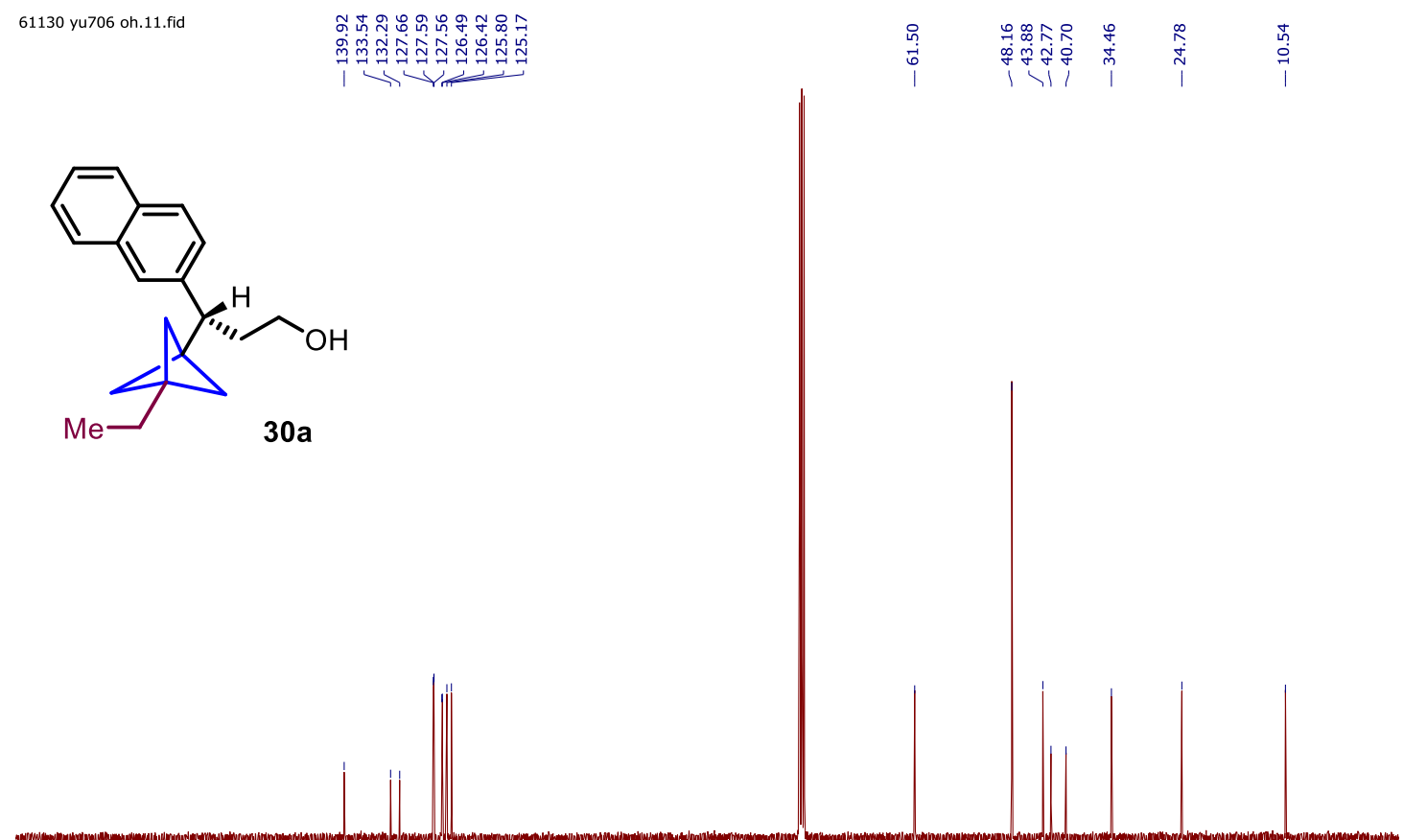

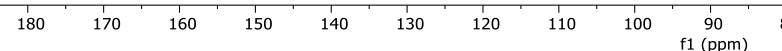


${ }^{1} \mathrm{H}$ NMR (400 MHz, $\mathrm{CDCl}_{3}$ ) spectrum for product 31a (see procedure):

60192 yu682.10.fid

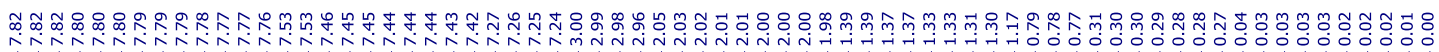<smiles></smiles>
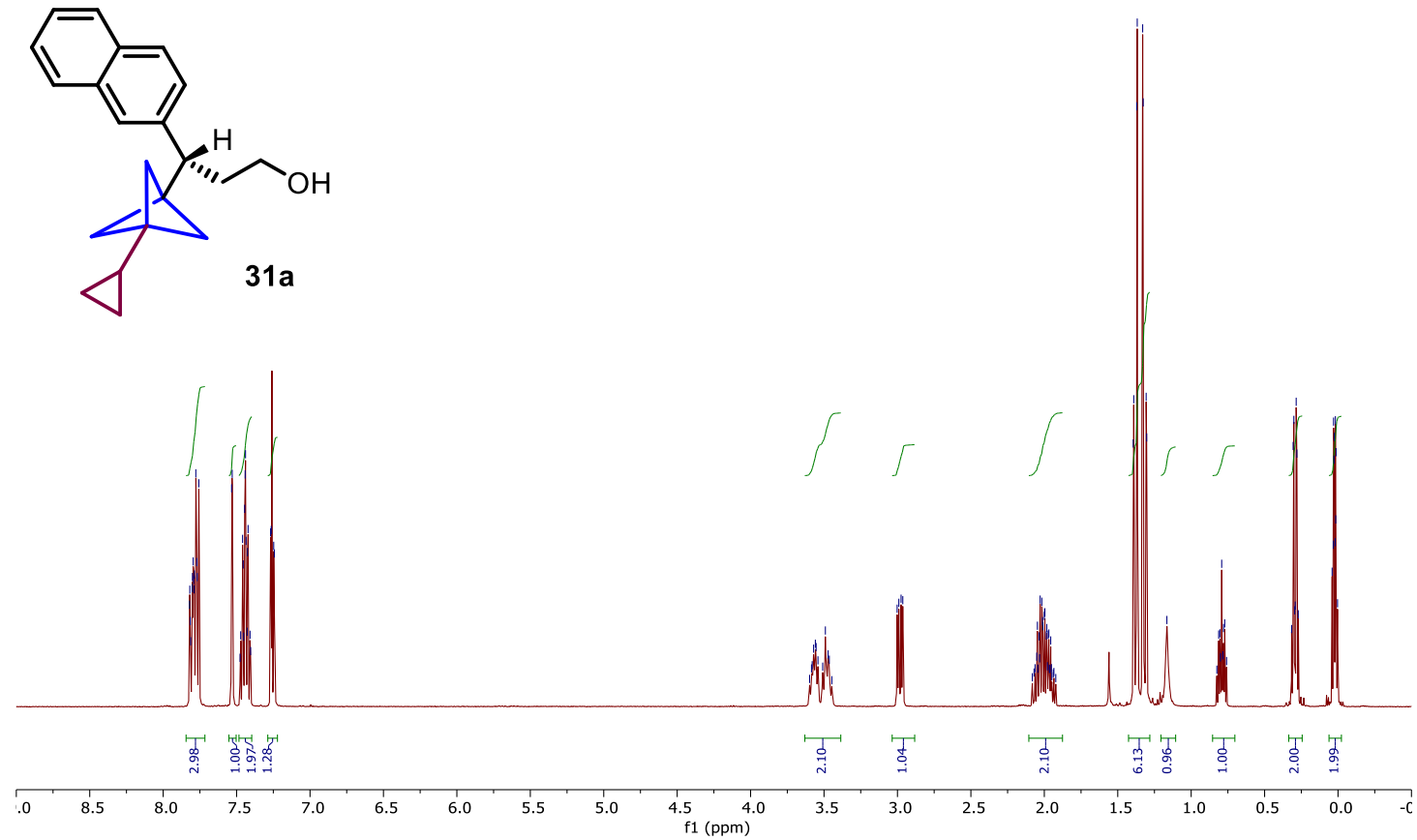

${ }^{13} \mathrm{C} \mathrm{NMR}\left(100 \mathrm{MHz}, \mathrm{CDCl}_{3}\right)$ spectrum for product 31a (see procedure):
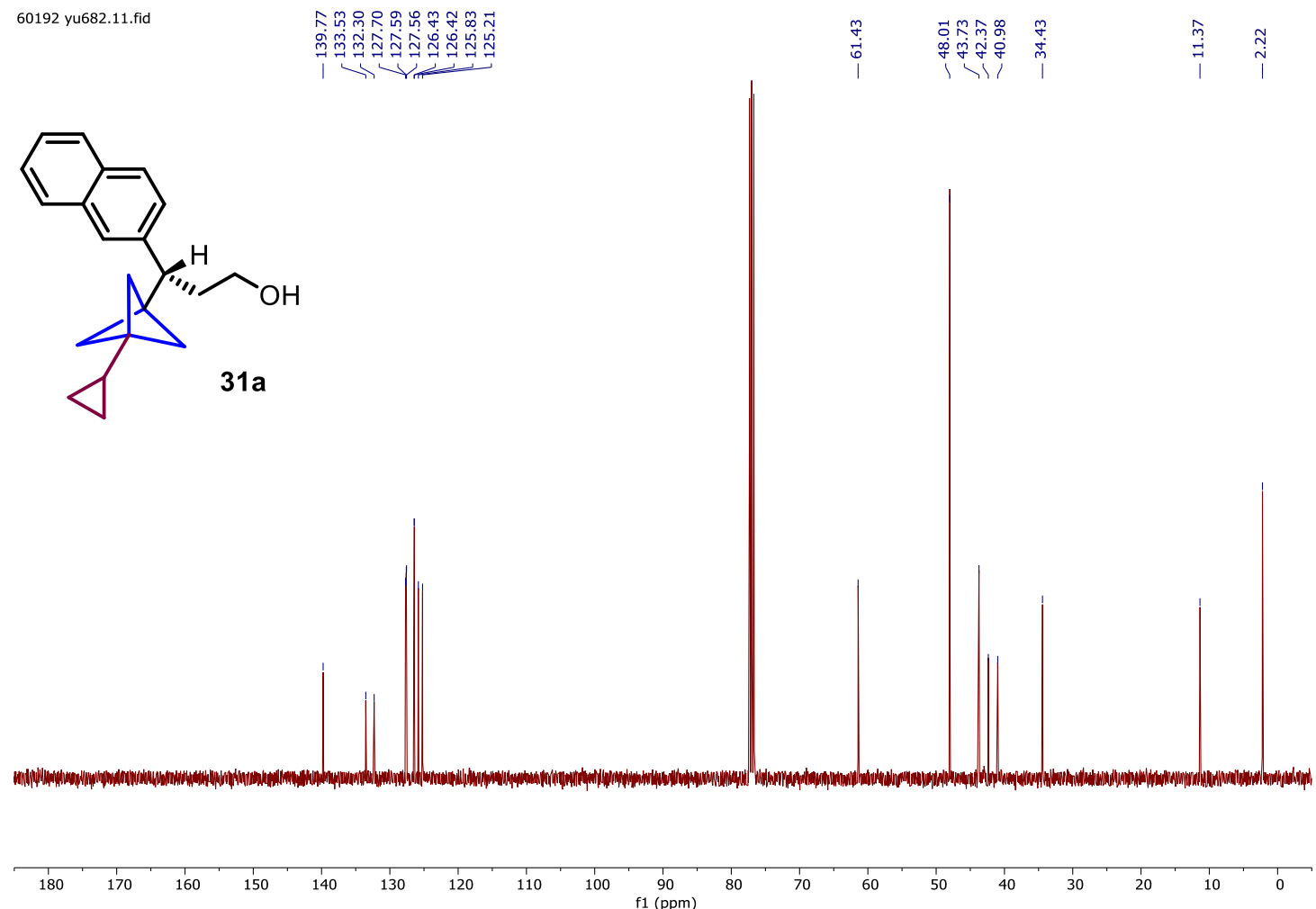
${ }^{1} \mathrm{H}$ NMR (400 MHz, $\mathrm{CDCl}_{3}$ ) spectrum for product 32a (see procedure):

61306 yu712 oh.10.fid

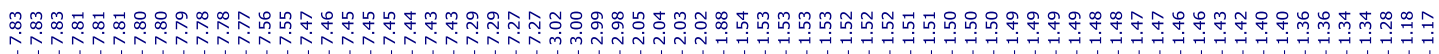

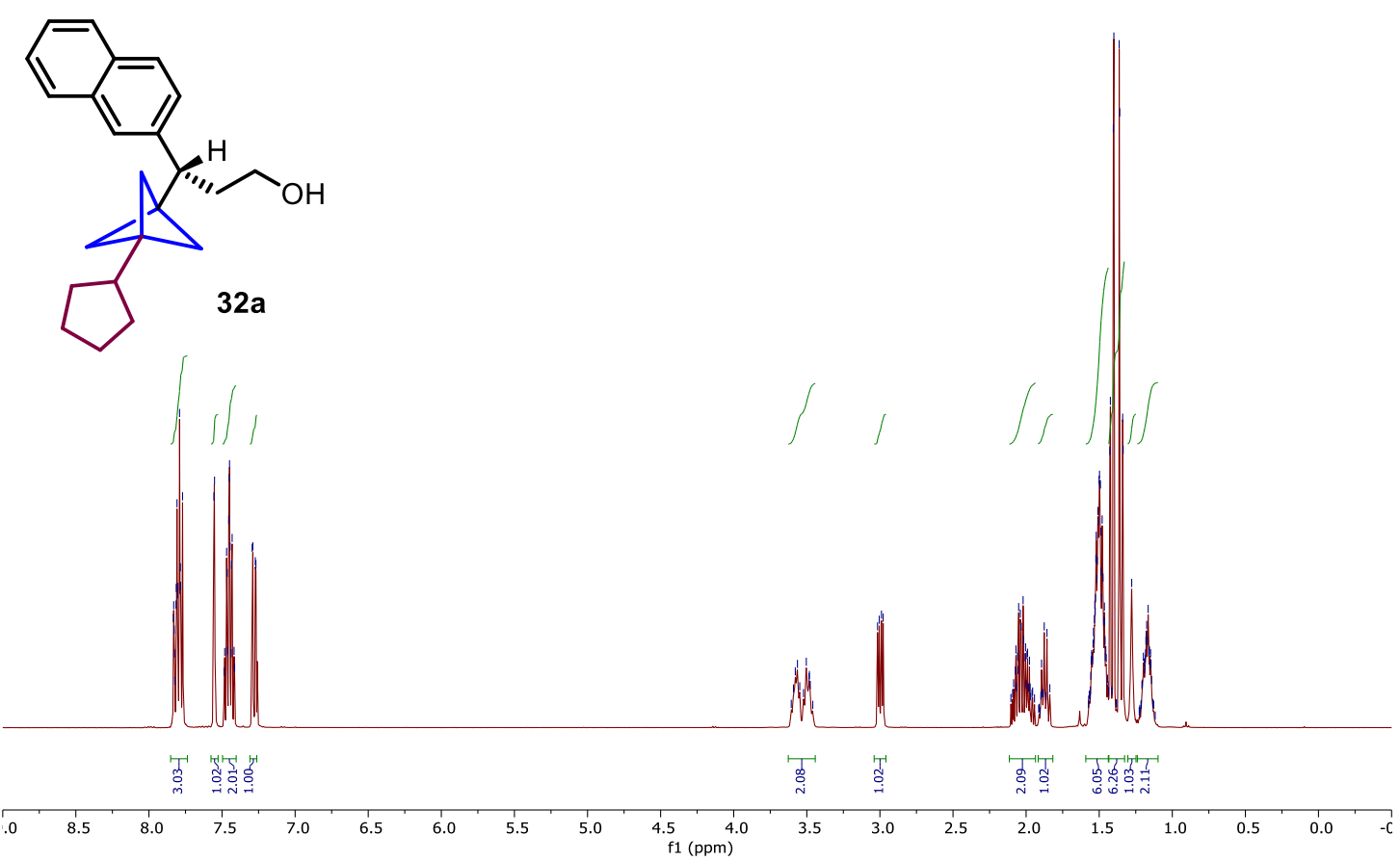

${ }^{13} \mathrm{C} \mathrm{NMR}\left(100 \mathrm{MHz}, \mathrm{CDCl}_{3}\right)$ spectrum for product 32a (see procedure): 61306 yu712 oh.11.fid

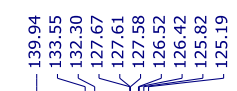

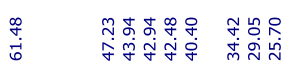

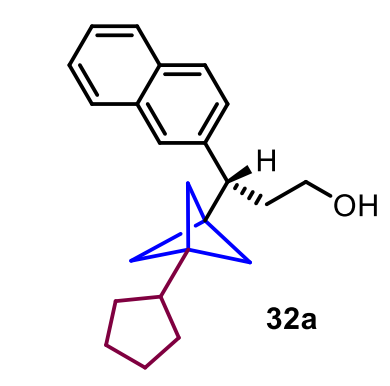
1
.42
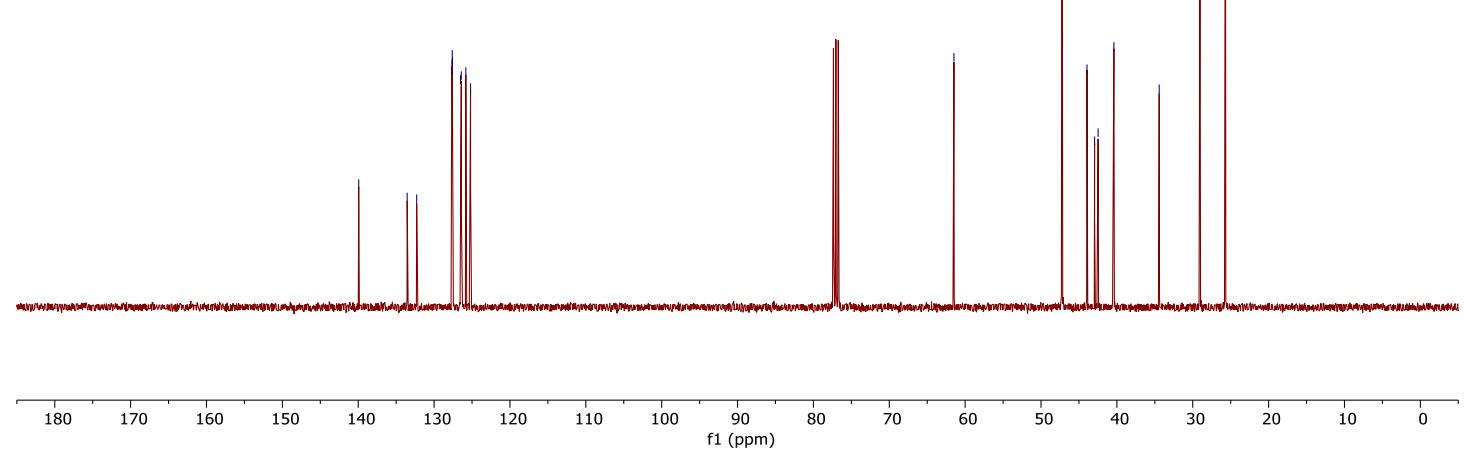
${ }^{1} \mathrm{H}$ NMR (400 MHz, $\mathrm{CDCl}_{3}$ ) spectrum for product 34 (see procedure):

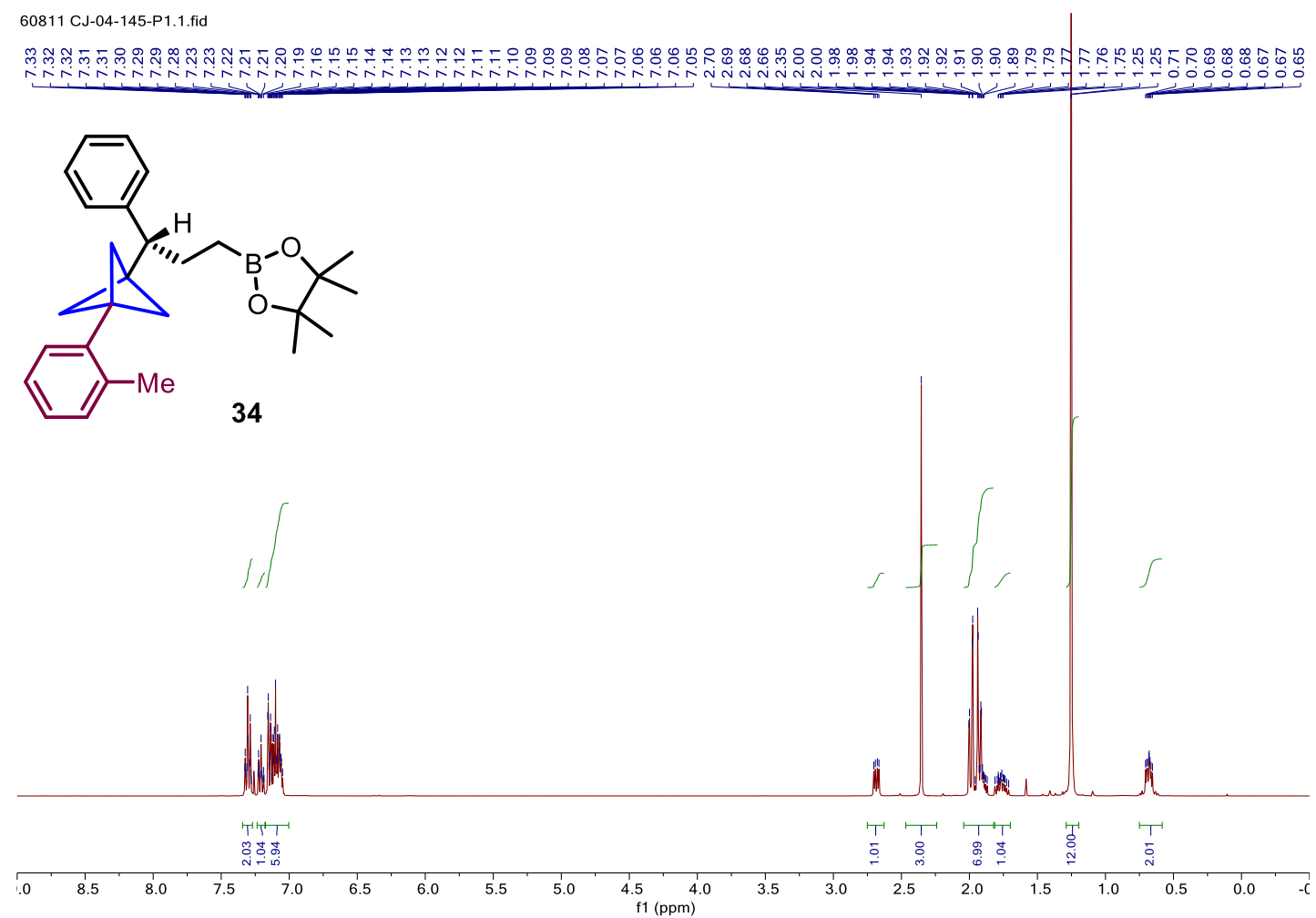

${ }^{11} \mathrm{~B}$ NMR (128 MHz, $\mathrm{CDCl}_{3}$ ) spectrum for product 34 (see procedure):

va/cj17242 CJ-04-145-P1

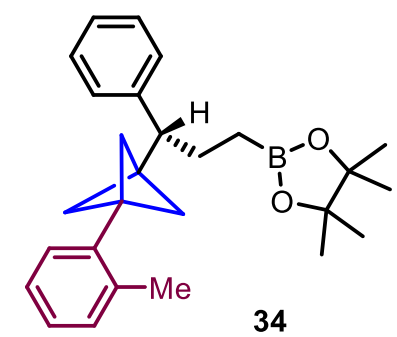

34

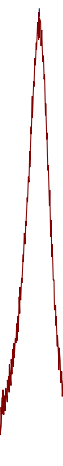


${ }^{13} \mathrm{C} \mathrm{NMR}\left(100 \mathrm{MHz}, \mathrm{CDCl}_{3}\right.$ ) spectrum for product 34 (see procedure):

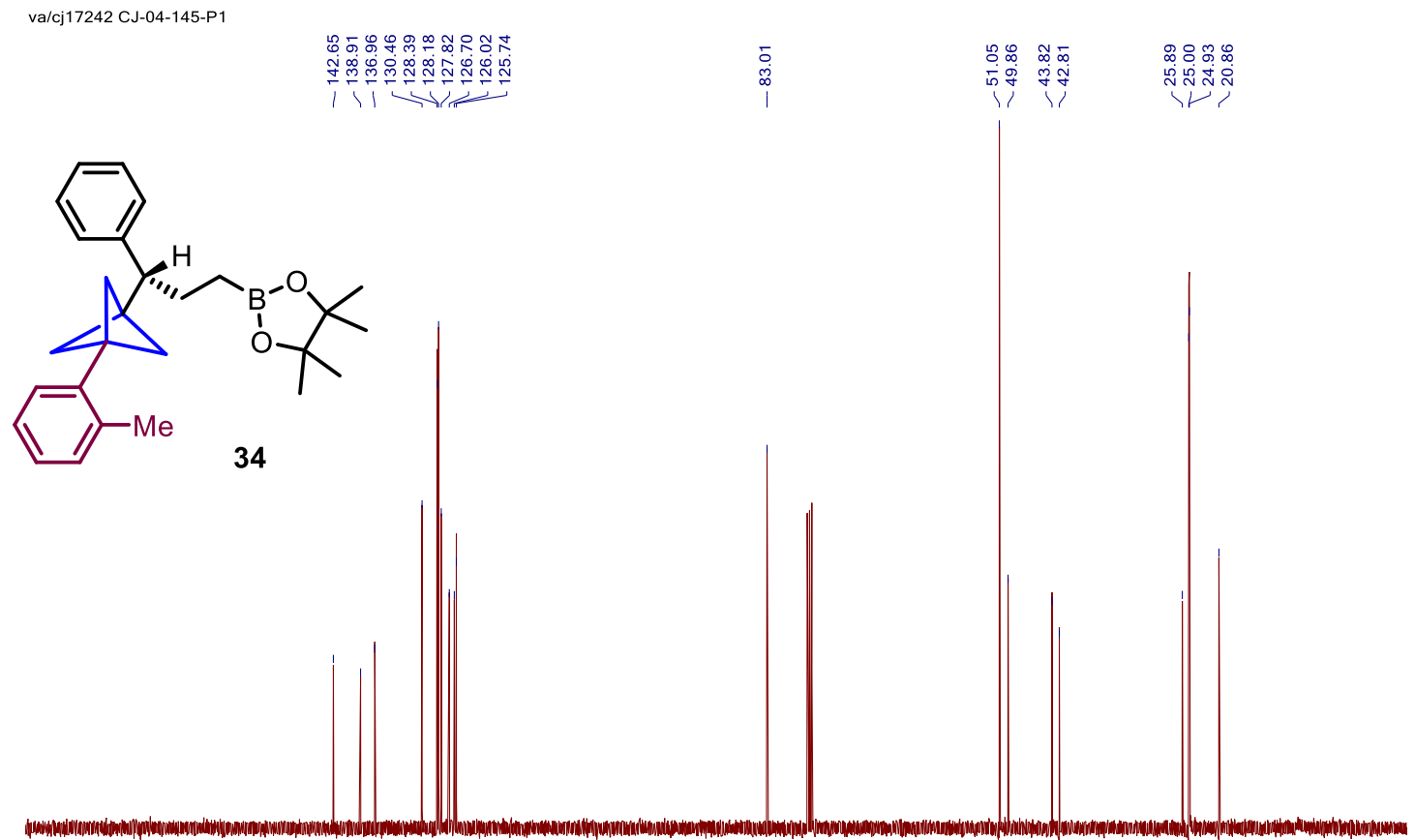

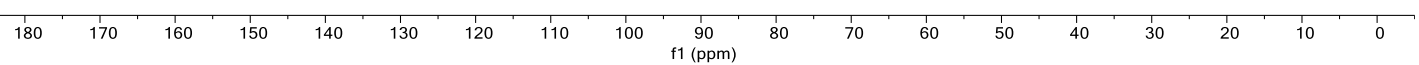


${ }^{1} \mathrm{H}$ NMR (400 MHz, $\mathrm{CDCl}_{3}$ ) spectrum for product 34a (see procedure):

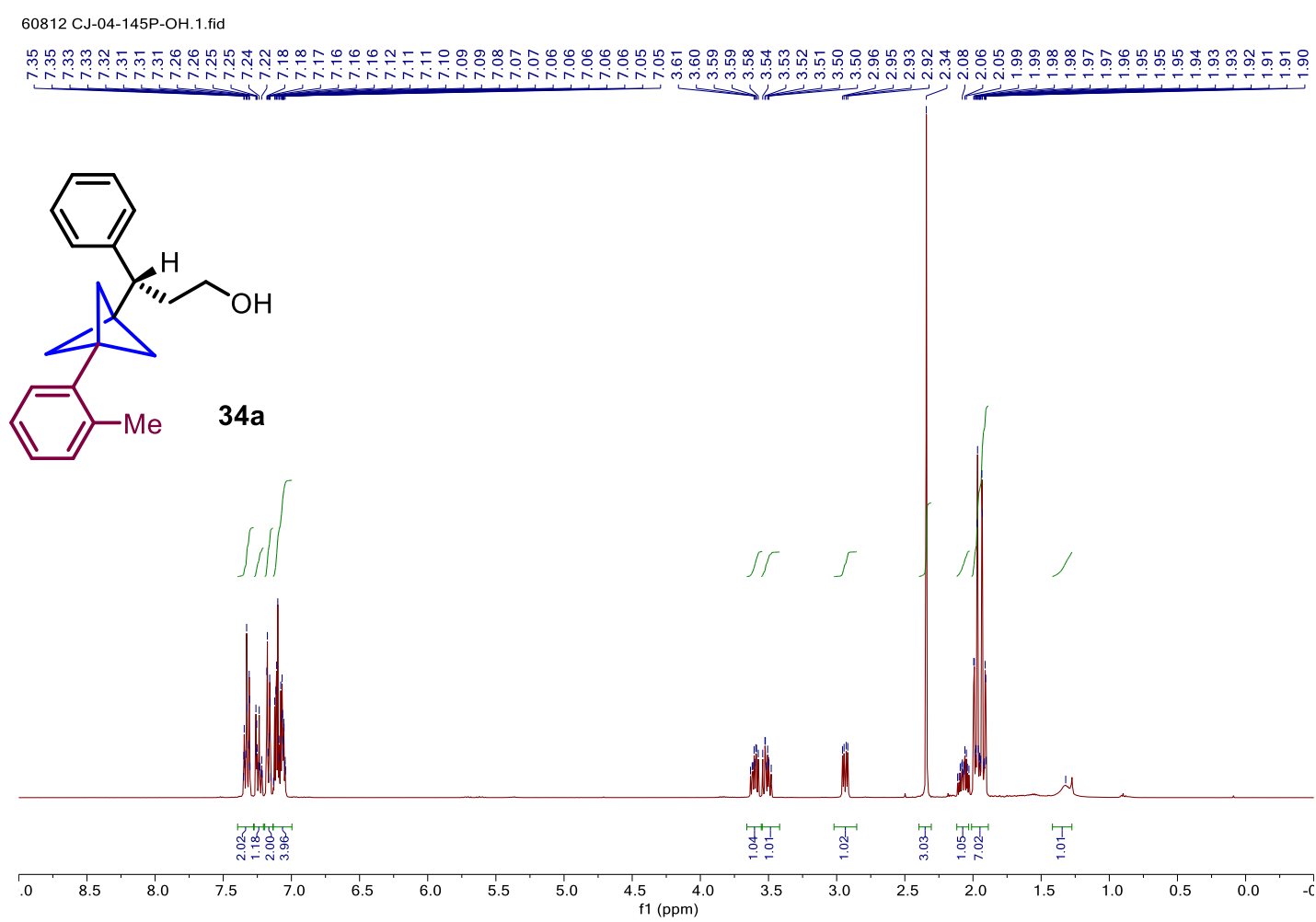

${ }^{13} \mathrm{C}$ NMR $\left(100 \mathrm{MHz}, \mathrm{CDCl}_{3}\right)$ spectrum for product $\mathbf{3 4 a}$ (see procedure): va/cj17242 CJ-04-145P-OH
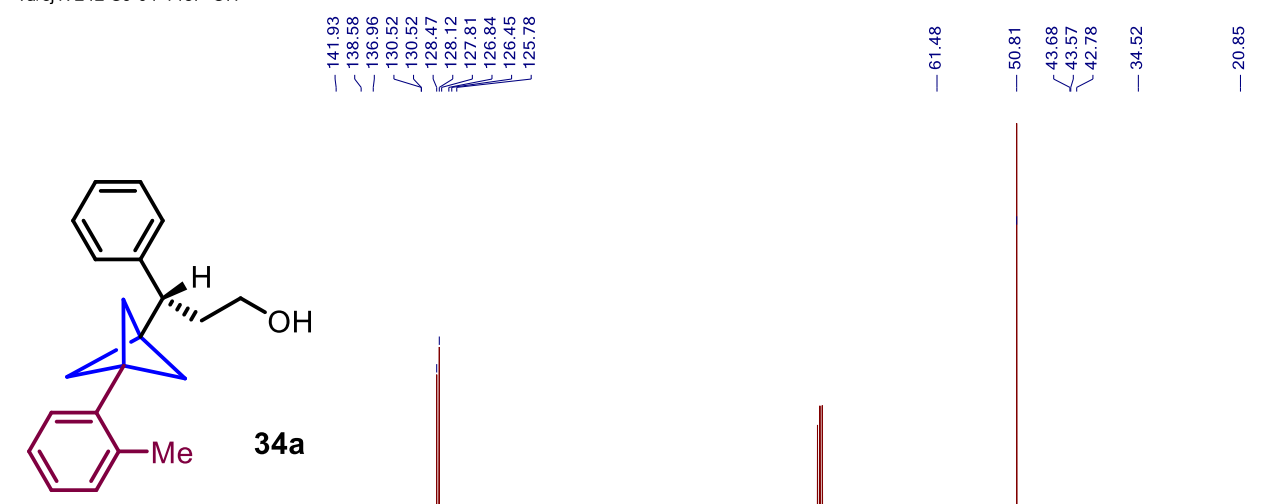

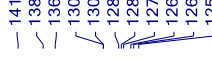

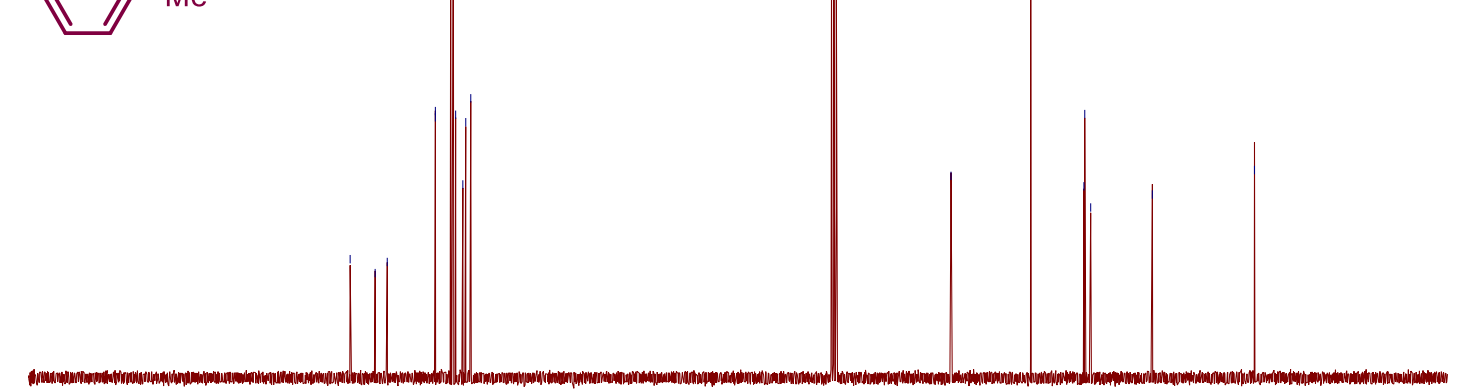

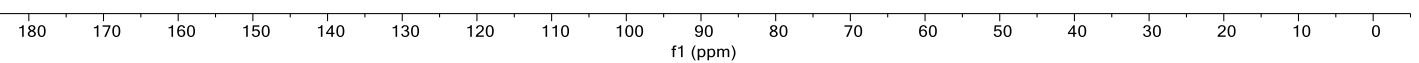


${ }^{1} \mathrm{H}$ NMR (400 MHz, acetone- $d_{6}$ ) spectrum for product 35 (see procedure): 61657 yu729.10.fid

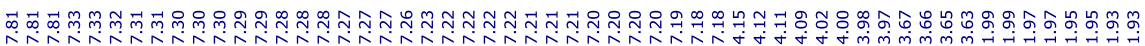

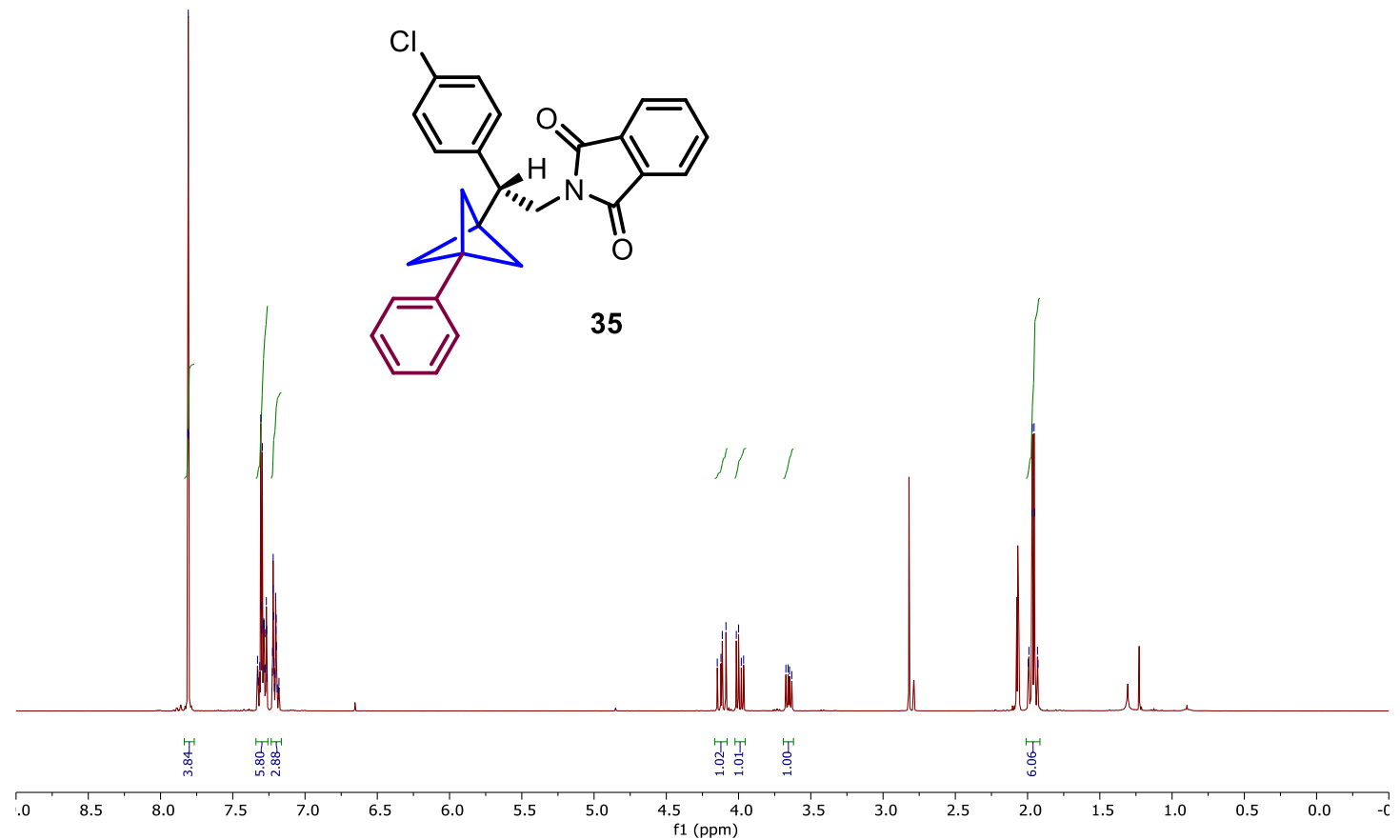

${ }^{13} \mathrm{C}$ NMR (100 MHz, acetone- $d_{6}$ ) spectrum for product 35 (see procedure):

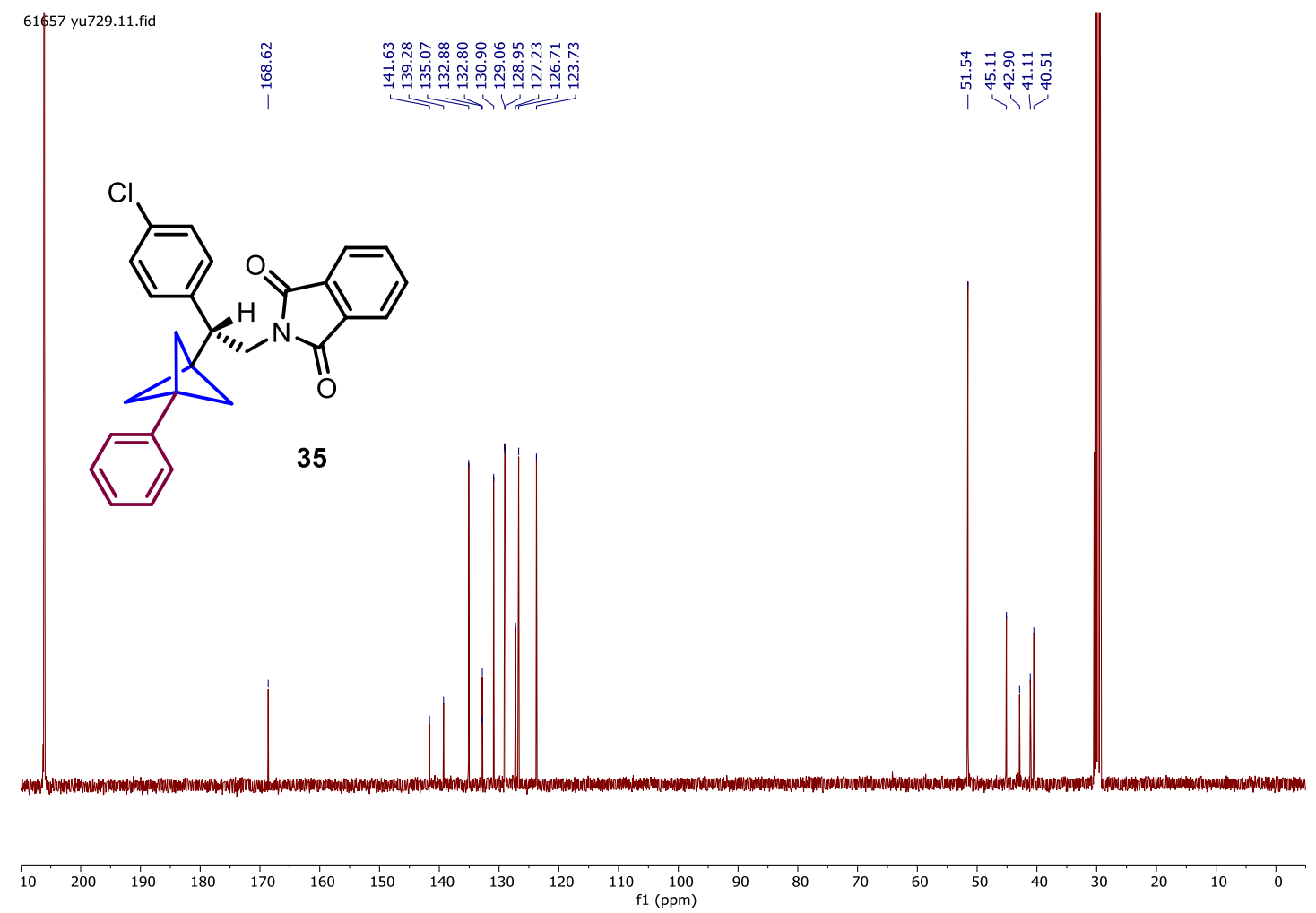


${ }^{1} \mathrm{H}$ NMR (400 MHz, $\mathrm{CDCl}_{3}$ ) spectrum for product 36 (see procedure):

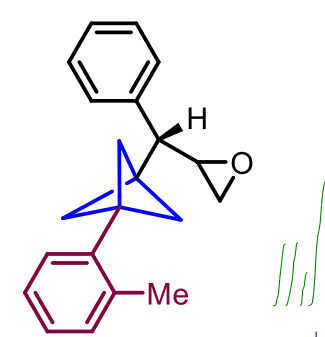

36

$54: 46 d r$

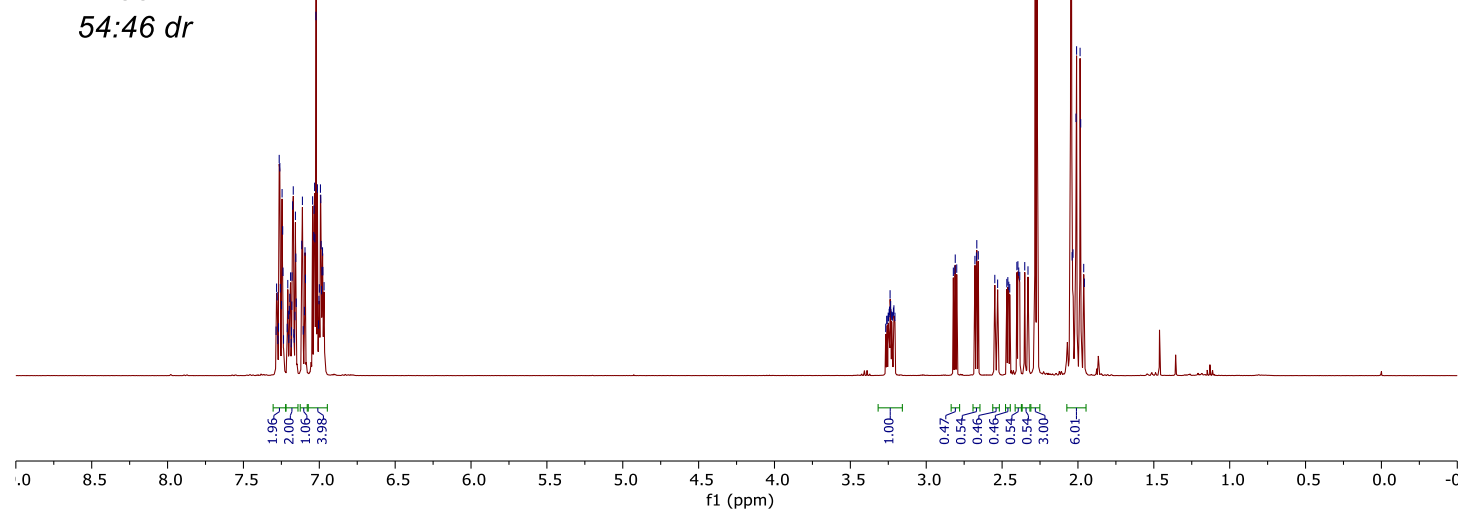

${ }^{13} \mathrm{C}$ NMR (100 MHz, $\mathrm{CDCl}_{3}$ ) spectrum for product 36 (see procedure):

61489 yu717.11.fid

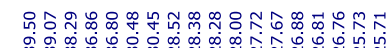

$$
\begin{aligned}
& \text { 。 }
\end{aligned}
$$

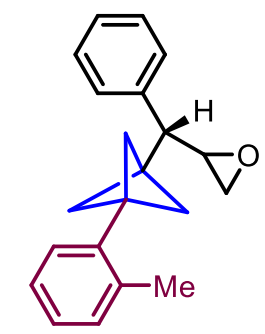

36

$54: 46 d r$
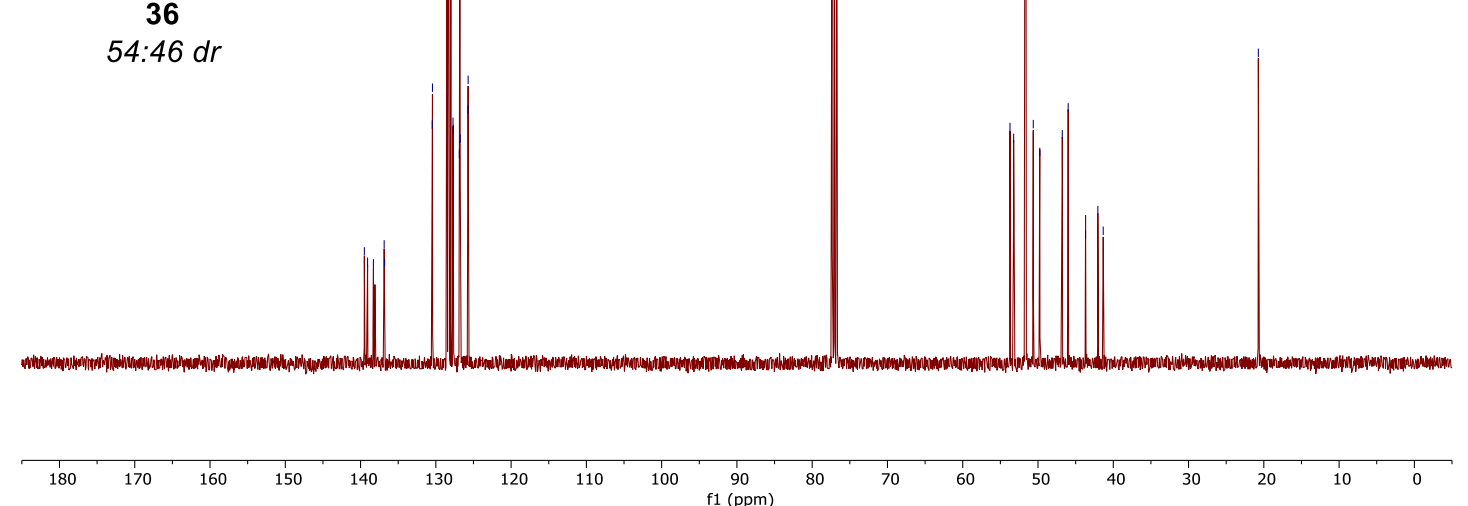
${ }^{1} \mathrm{H}$ NMR (400 MHz, $\mathrm{CDCl}_{3}$ ) spectrum for product 37 (see procedure):

61490 CJ-04-150P.1.fid

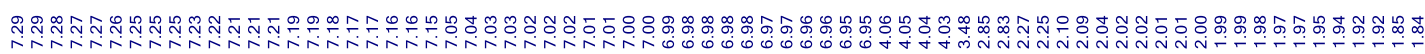
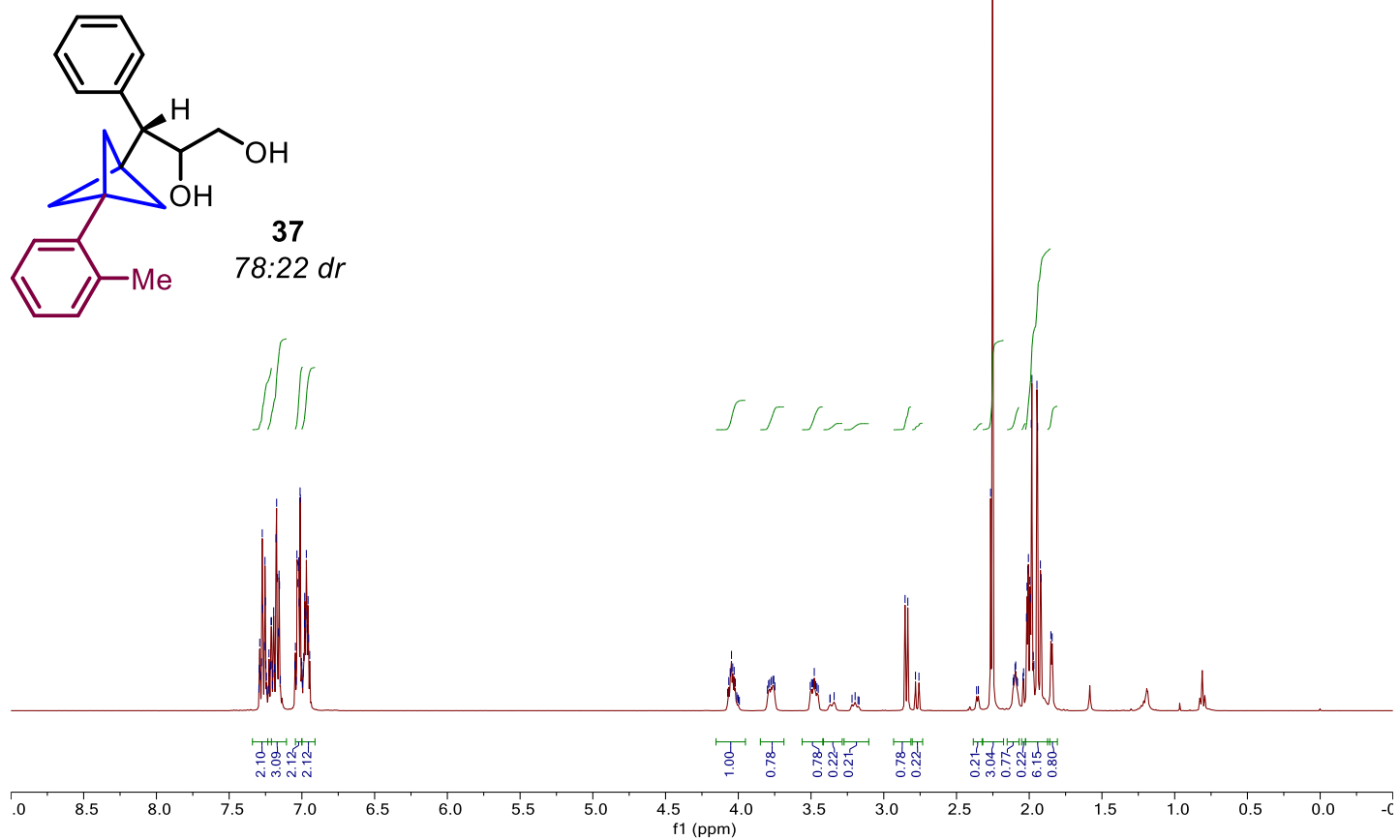

${ }^{13} \mathrm{C}$ NMR (100 MHz, $\mathrm{CDCl}_{3}$ ) spectrum for product 37 (see procedure):

61490 CJ-04-150P.10.fid

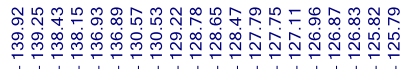
约
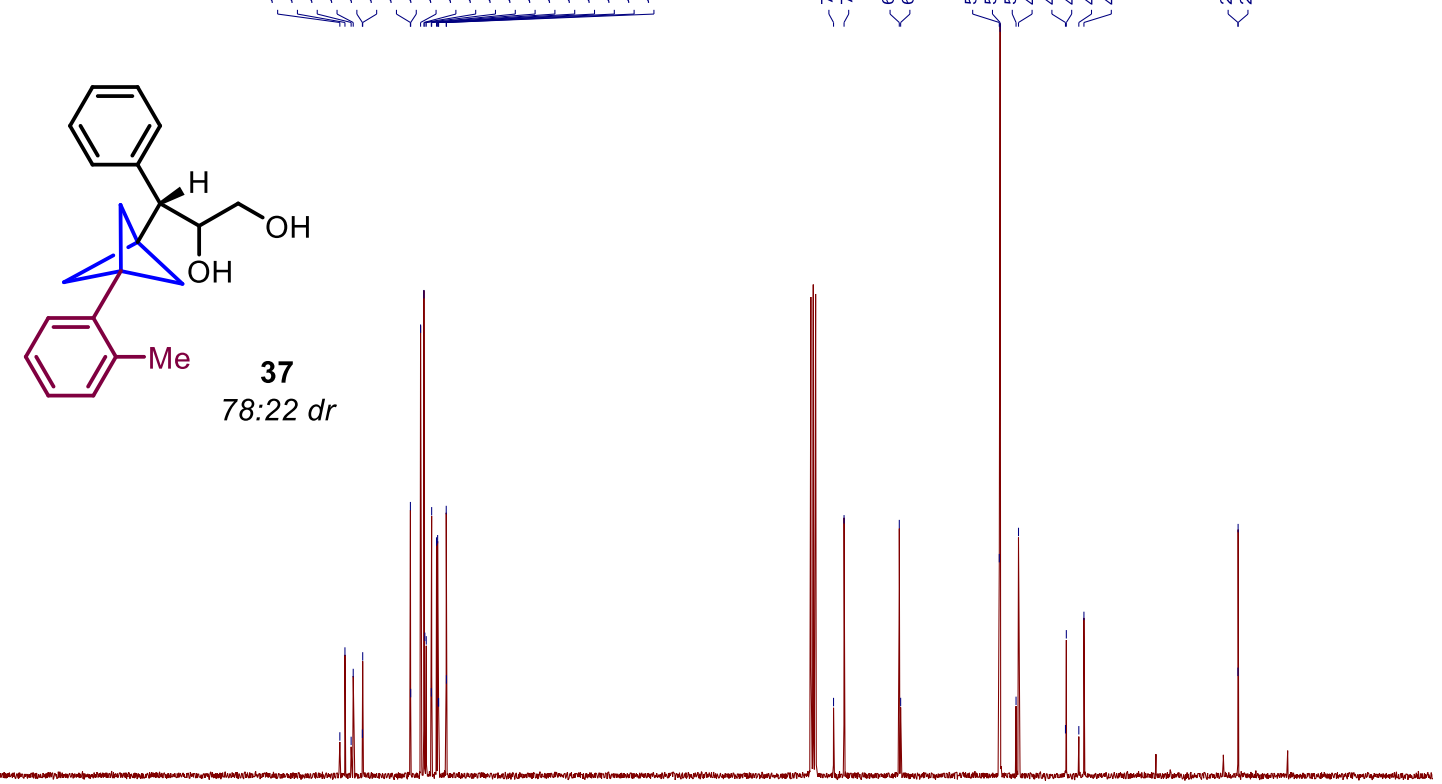
${ }^{1} \mathrm{H}$ NMR (400 MHz, $\mathrm{CDCl}_{3}$ ) spectrum for product 38 (see procedure):

61488 yu716.10.fid

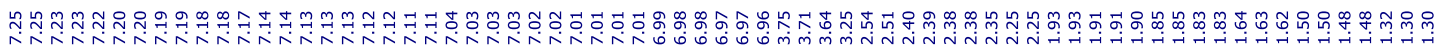
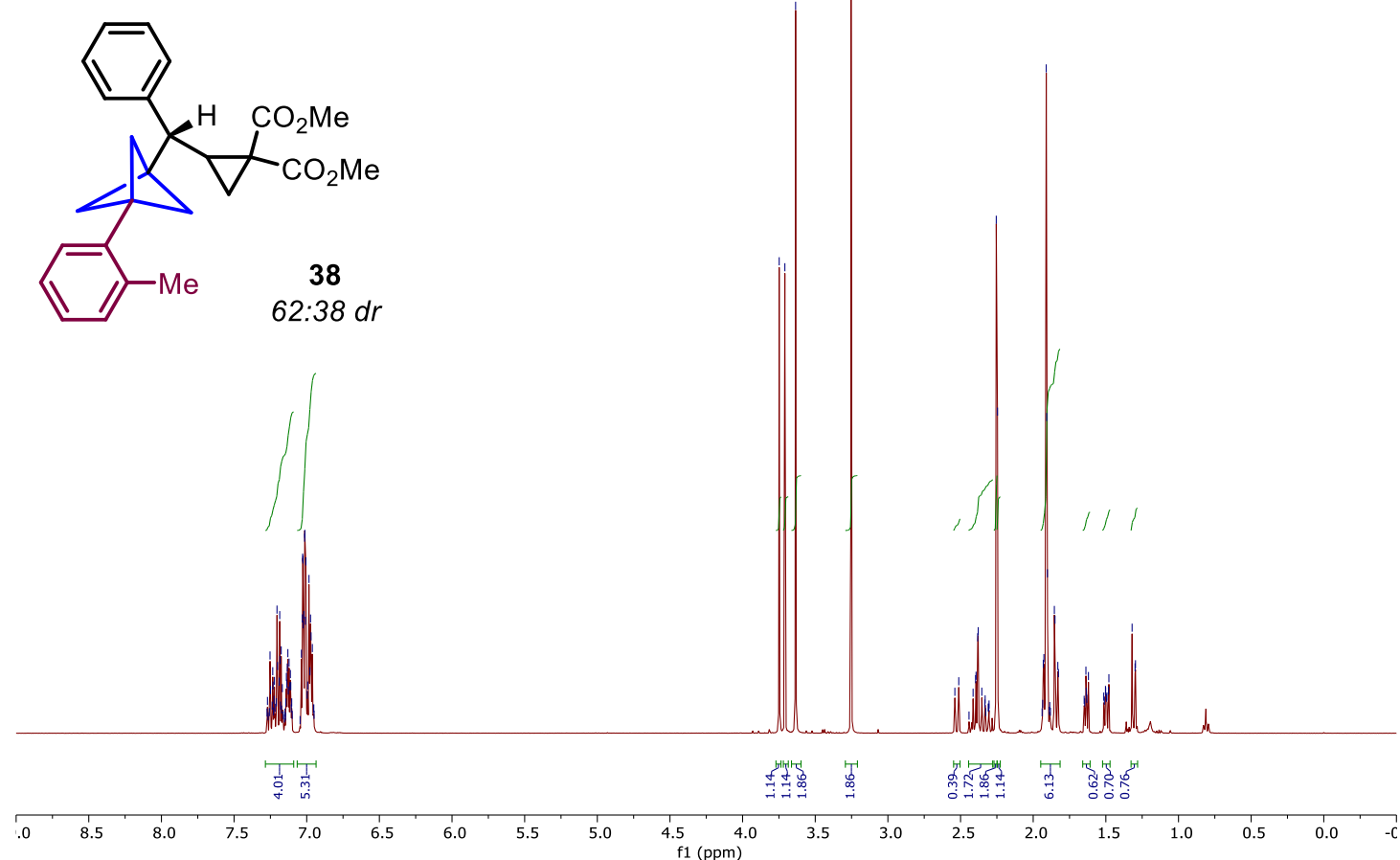

${ }^{13} \mathrm{C} \mathrm{NMR}\left(100 \mathrm{MHz}, \mathrm{CDCl}_{3}\right.$ ) spectrum for product 38 (see procedure):

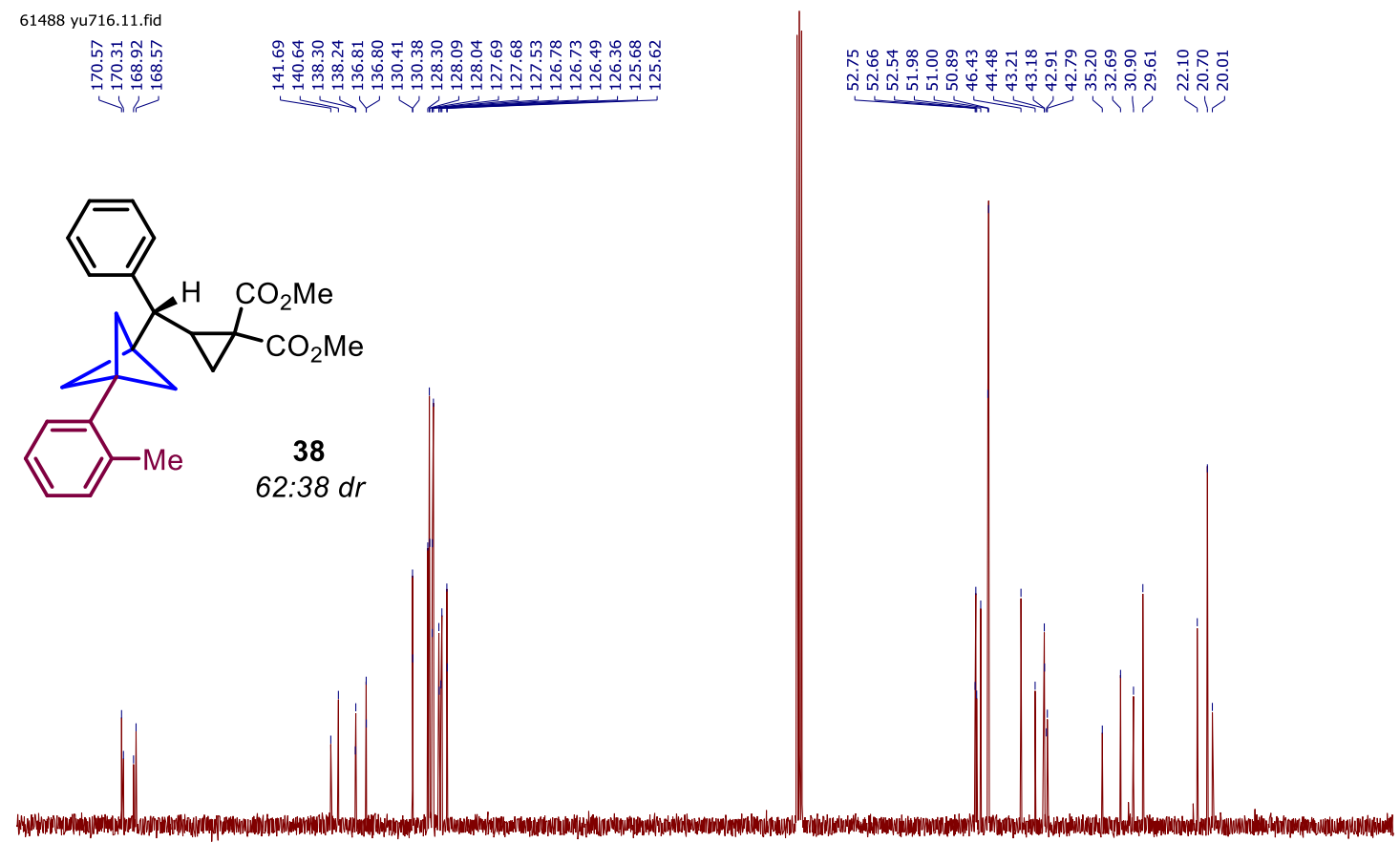

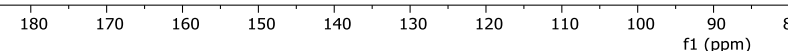


${ }^{1} \mathrm{H}$ NMR (400 MHz, $\mathrm{CDCl}_{3}$ ) spectrum for product 39 (see procedure):

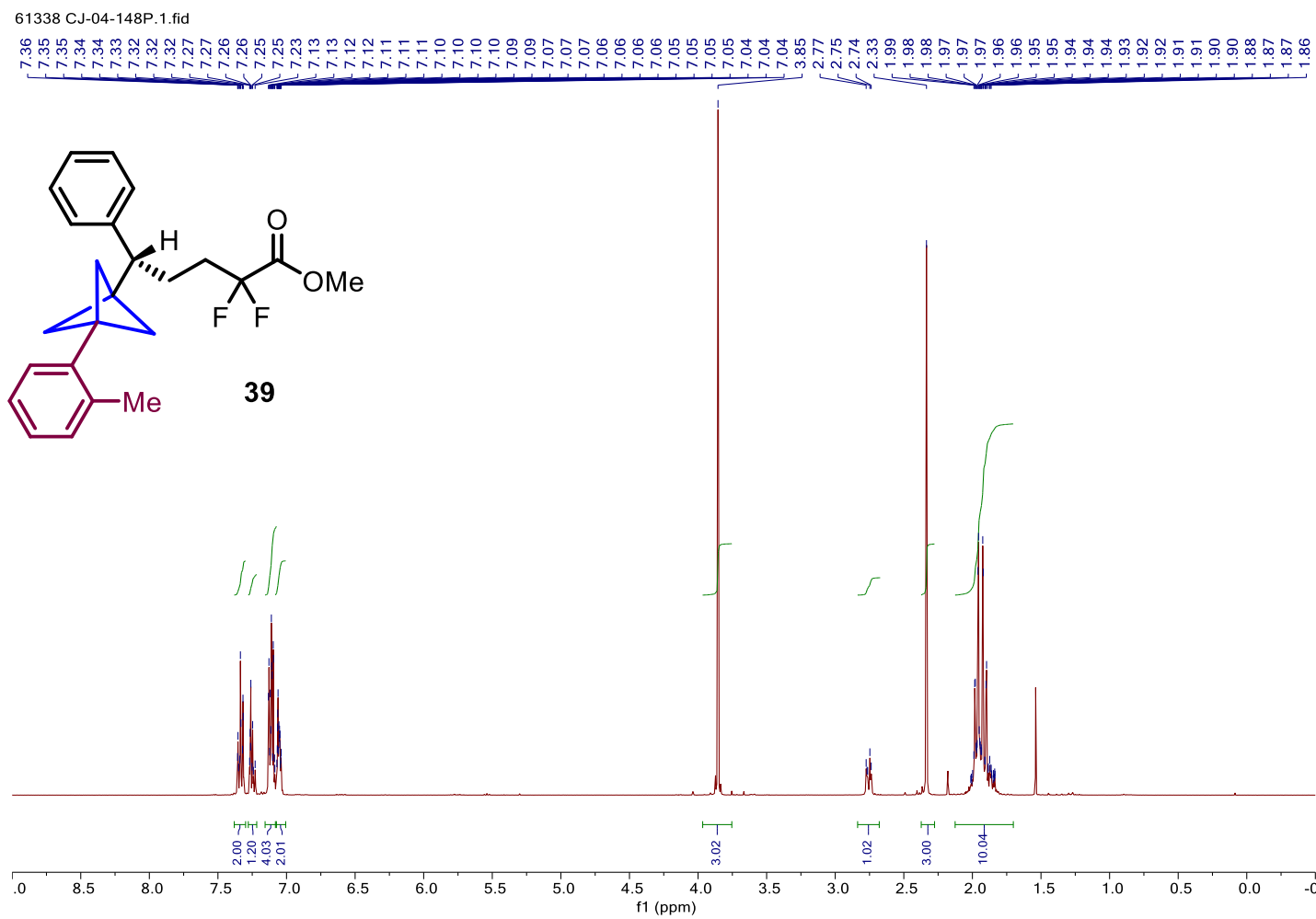

${ }^{19} \mathrm{~F} \mathrm{NMR}\left(377 \mathrm{MHz}, \mathrm{CDCl}_{3}\right.$ ) spectrum for product 39 (see procedure):

61338 CJ-04-148P.2.fid

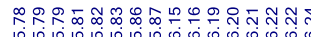

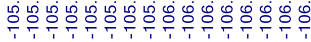

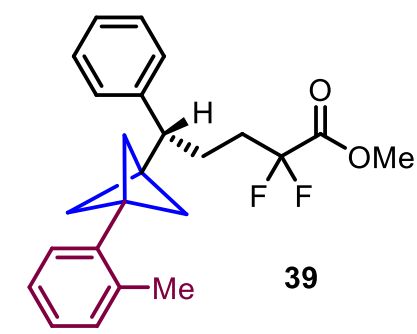

8

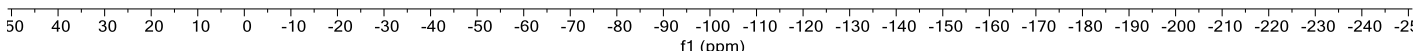


${ }^{13} \mathrm{C} \mathrm{NMR}\left(100 \mathrm{MHz}, \mathrm{CDCl}_{3}\right.$ ) spectrum for product 39 (see procedure):
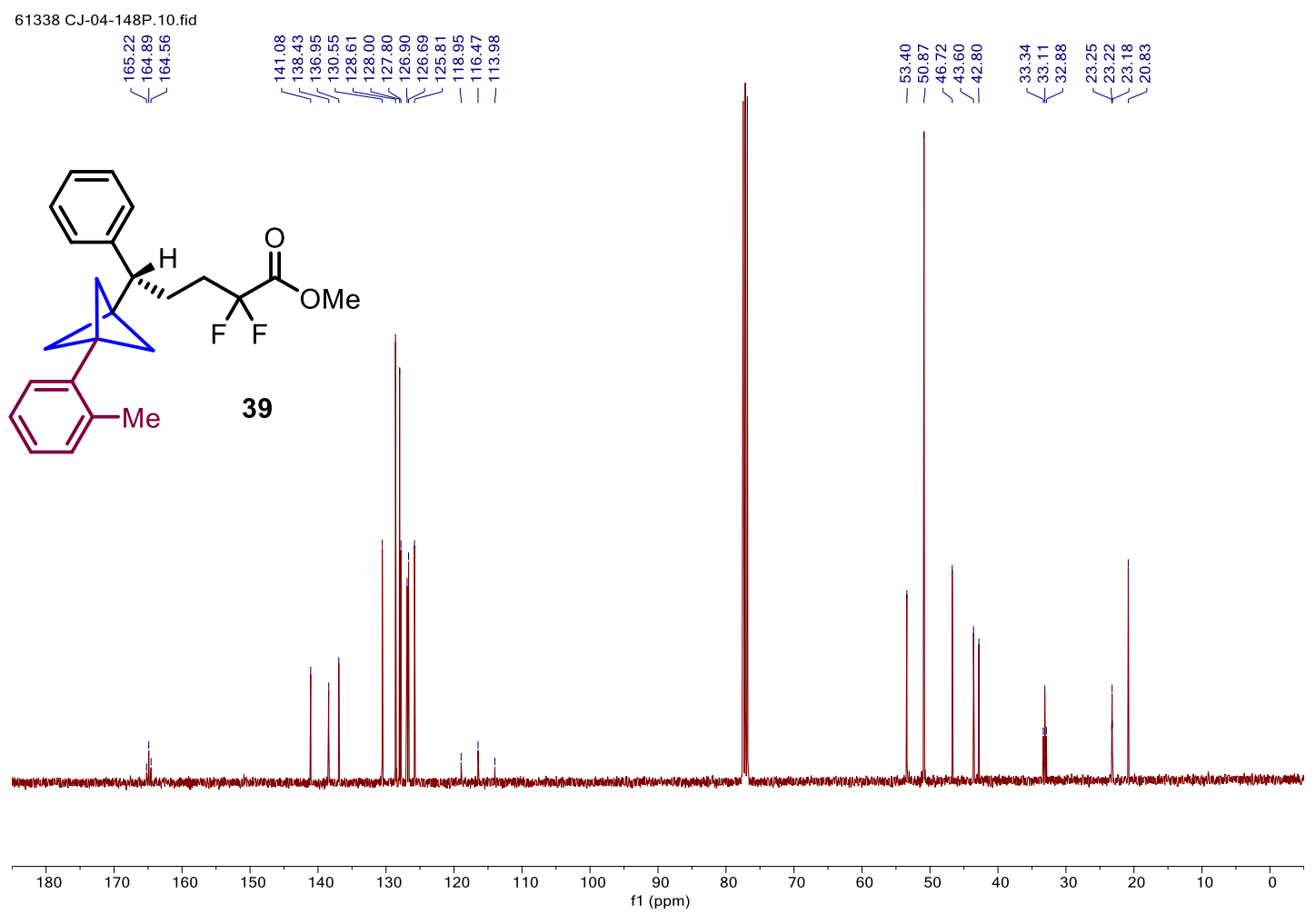
${ }^{1} \mathrm{H}$ NMR (400 MHz, $\mathrm{CDCl}_{3}$ ) spectrum for product 40 (see procedure):

60539 yu693.10.fid

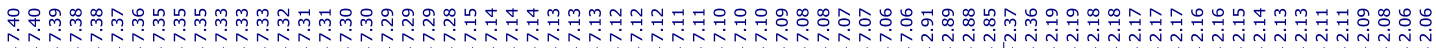
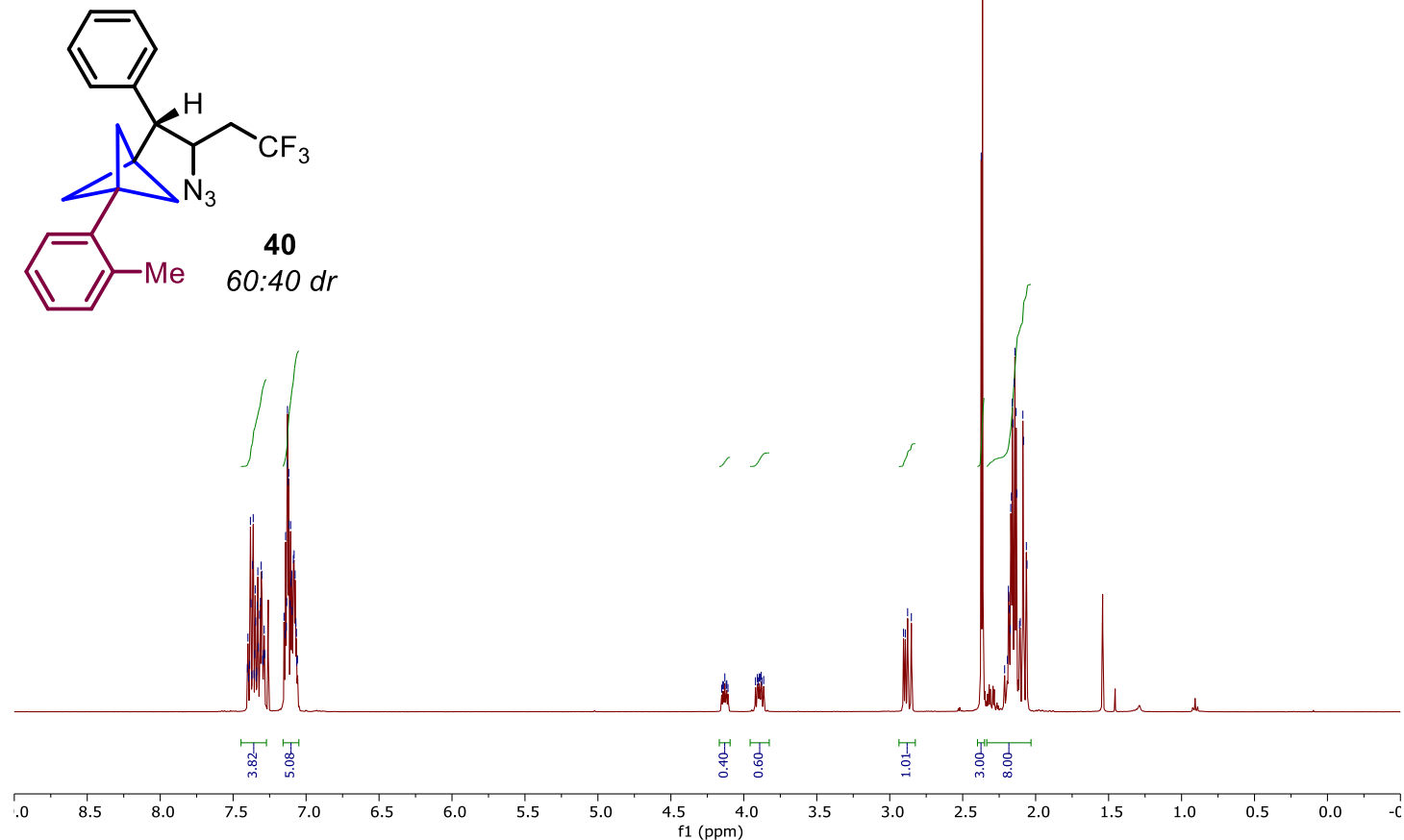

${ }^{19} \mathrm{~F} \mathrm{NMR}\left(377 \mathrm{MHz}, \mathrm{CDCl}_{3}\right)$ spectrum for product 40 (see procedure):

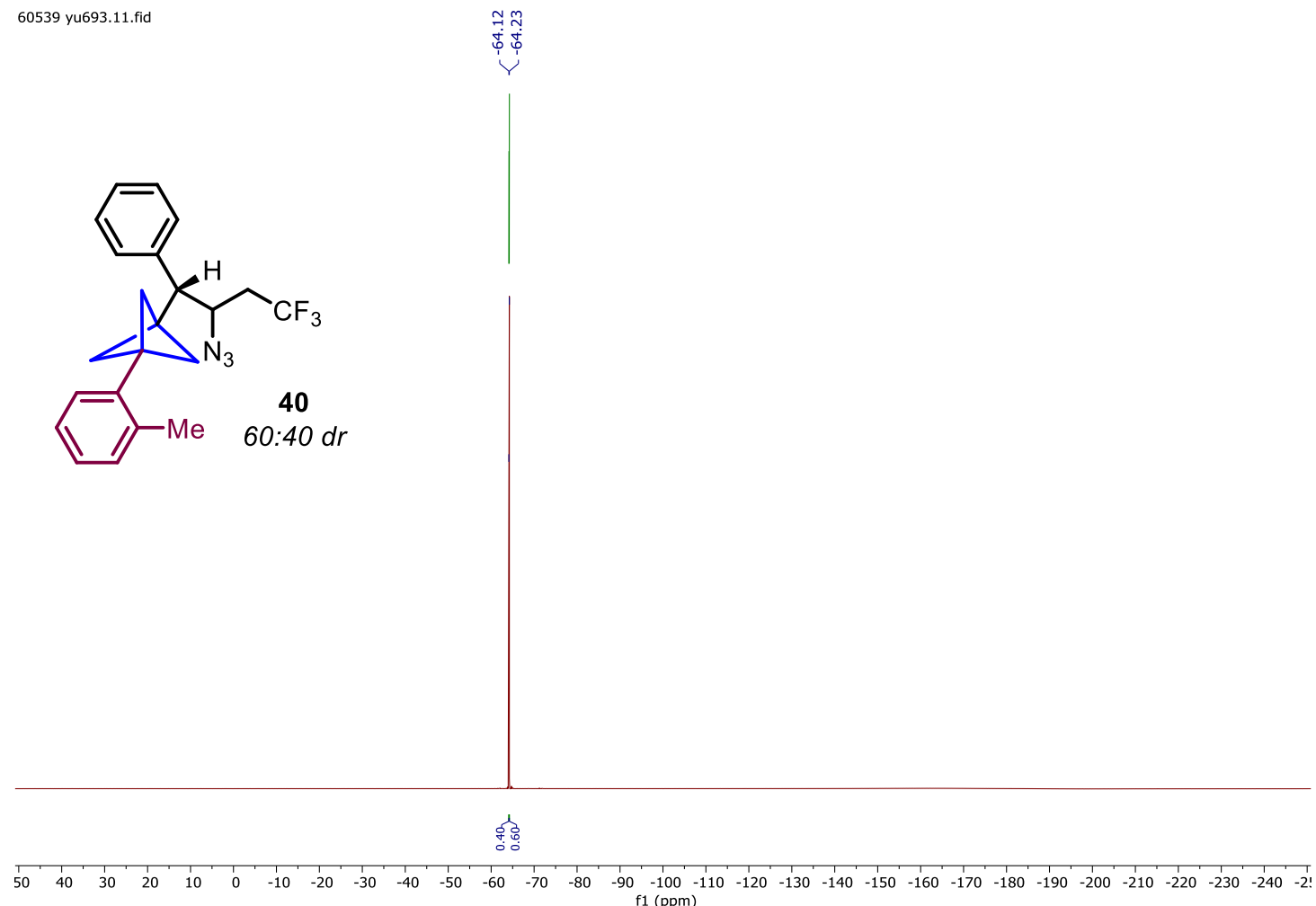


${ }^{13} \mathrm{C} \mathrm{NMR}\left(100 \mathrm{MHz}, \mathrm{CDCl}_{3}\right.$ ) spectrum for product 40 (see procedure):

60539 yu693.12.fid

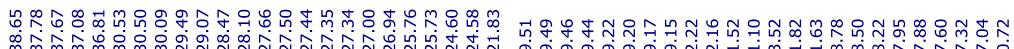

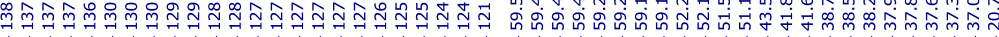
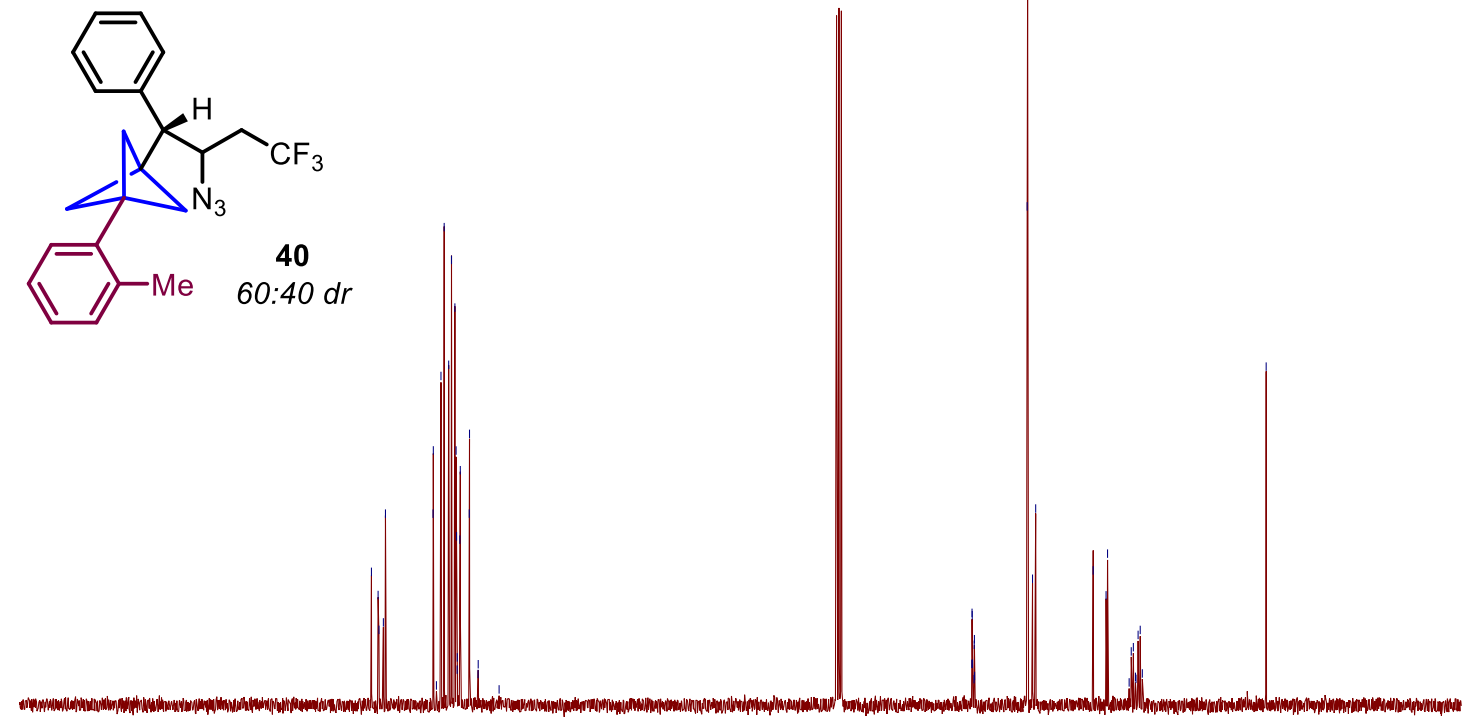

$180 \quad 170 \quad 160$

140

90
$\mathrm{f} 1(\mathrm{ppm})$ 